\title{
The Use of a BIM-Based Framework to Support Safe Facility Management Processes
}

\author{
Eric Michael Wetzel
}

Dissertation submitted to the faculty of the Virginia Polytechnic Institute and State University in partial fulfillment of the requirements for the degree of

Doctor of Philosophy

In

Environmental Design and Planning

Walid Y. Thabet, Chair

Tanyel Bulbul

Jason D. Lucas

Thomas Mills

November 7, 2016

Blacksburg, VA

Keywords: Building Information Modeling (BIM), Facility Management, Safety, Asset O\&M 
The Use of a BIM-Based Framework to Support Safe Facility Management Processes

Eric Michael Wetzel

ACADEMIC ABSTRACT

According to the United States Bureau of Labor Statistics, 293 people lost their life from 2008-2012 in the field of Facility Management (FM). In that same timeframe, private employers recorded 98,420 cases of occupational injuries and illness, with 26,190 cases requiring a minimum of 31 days away from work. Workers in this field are at constant risk of electrical shock, falls, crushing, cuts, and bruises and as a result, have a much higher rate of injury and illness than the national average.

Case study analysis confirms that many of the recorded accidents could have been avoided had the victim followed appropriate hazard mitigation steps to safely execute a facility repair and maintenance task, defined in this research as safety protocol. Currently, safety related information is conveyed to FM staff through training seminars, O\&M manuals, plans \& specifications, database storage, safety meetings, and safety literature. This information, although comprehensive, often remains fragmented among multiple resources and is left up to the worker's discretion whether the information is relevant. Research has shown that the more time and effort, known as inconvenience, an individual must spend obtaining information, the less likely they are to retrieve the information and obey the stated warnings.

This research focuses on the identification, categorization, transference, and delivery of safety related information applicable to facility management staff. This is executed by, obtaining safety inputs through various mechanisms of data collection, categorizing the safety inputs, transferring the information utilizing existing BIM-based software and research methods into a data storage repository, and designing a data retrieval and processing system (DRPS), integrated into the repository to interact with the data. The DRPS adds structure and relationships through a UML Class Diagram and Sequence Diagram. Additionally, standardized safety properties are developed for asset groups using the Asset Safety Identification Tool (ASIT). Finally, a conceptual graphical user interface (GUI) is developed to represent the interaction between the DRPS and the FM Worker. By combining the DRPS with a GUI, a FM worker can efficiently interact with a singular repository for safety information, eliminating the need to reference multiple resources in order to obtain comprehensive safety information. The goal of this research is to mitigate the fragmentation and timing inefficiencies within safety related information retrieval by developing a BIMbased framework to categorize, consolidate, and deliver job specific safety information, eliminating the need to reference multiple documents in order to develop a comprehensive, task specific safety plan. 
The Use of a BIM-Based Framework to Support Safe Facility Management Processes

Eric Michael Wetzel

PUBLIC ABSTRACT

According to the United States Bureau of Labor Statistics, 293 people lost their life from 2008-2012 in the field of Facility Management (FM). In that same timeframe, private employers recorded 98,420 cases of occupational injuries and illness, with 26,190 cases requiring a minimum of 31 days away from work. Workers in this field are at constant risk of electrical shock, falls, crushing, cuts, and bruises and as a result, have a much higher rate of injury and illness than the national average.

Data analysis confirms that many of the recorded accidents could have been avoided had the victim followed appropriate hazard mitigation steps to safely execute a facility repair and maintenance task. Currently, safety related information is conveyed to FM staff through training seminars, operations \& maintenance manuals, plans \& specifications, virtual training, safety meetings, and safety literature. This information, although comprehensive, often remains fragmented among multiple resources and is left up to the worker's discretion whether the information is relevant. Research has shown that the more time and effort an individual must spend obtaining information, the less likely they are to retrieve the information and obey the stated warnings.

This research focuses on the identification, categorization, transference, and delivery of safety related information applicable to FM staff. This is executed by, obtaining safety inputs (categorized information) through various mechanisms of data collection, organizing the safety inputs, transferring the information utilizing existing engineering software and research methods into a computer based data storage repository, and designing a data retrieval and processing system (DRPS), integrated into the data storage repository to interact with the data. The DRPS adds structure and relationships through computer programming, using a Unified Modeling Language (UML) Class Diagram and Sequence Diagram. Additionally, standardized safety properties are developed for FM assets (operations equipment) using the Asset Safety Identification Tool (ASIT). Finally, a conceptual computer interface (GUI) is developed to represent the interaction between the DRPS and FM Worker. By combining the DRPS with a GUI, a FM worker can efficiently interact with a singular repository for safety information, eliminating the need to reference multiple resources in order to obtain comprehensive safety information. The goal of this research is to mitigate the fragmentation and timing inefficiencies with retrieving safety information by developing a computer based framework to categorize, consolidate, and deliver job specific safety information, eliminating the need to reference multiple documents in order to develop a comprehensive, task specific safety plan. 


\section{ACKNOWLEDGEMENTS}

The author would like to acknowledge the following people for their support during this research:

- His parents (Edward \& Veronica) and brother (Peter), who have been tremendous source of love, support, and encouragement

- His advisor and dissertation chair, Walid Thabet

- The members of the dissertation committee, Tanyel Bulbul, Jason Lucas, and Thom Mills

- The industry professionals who leant their time and expertise to the validation of this research

The author would like to acknowledge the Virginia Tech Department of Building Construction and the Cunningham Doctoral Award that is funding the work that is being executed in this document. The findings and conclusions in this document are those of the author, who is responsible for its content, and do not necessarily represent the views of Virginia Tech or the Cunningham Award. No statement in this dissertation should be construed as an official position of Virginia Tech or the Cunningham Award. 


\section{TABLE OF CONTENTS}

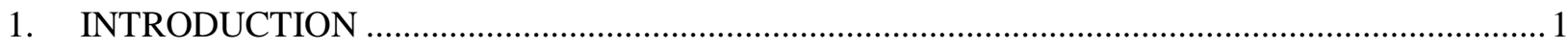

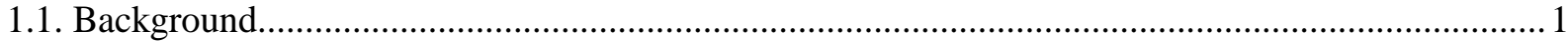

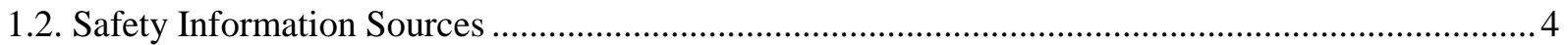

1.3. Fragmentation of Relevant Safety Information to Support Facility Management ...........................6

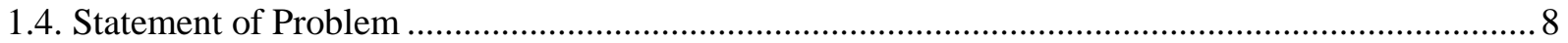

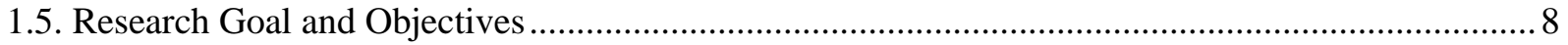

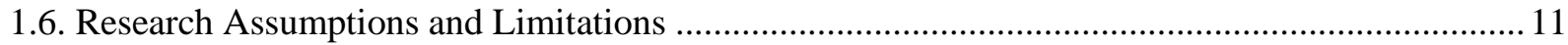

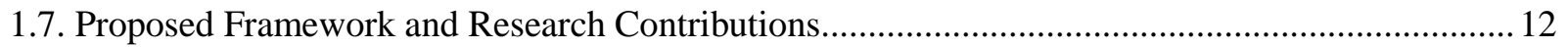

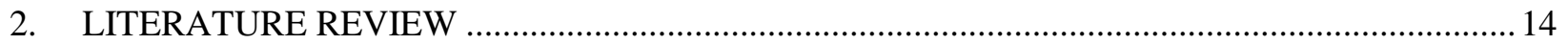

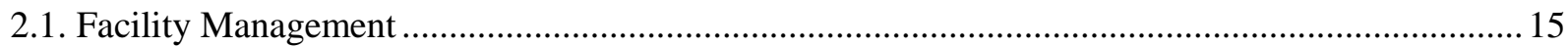

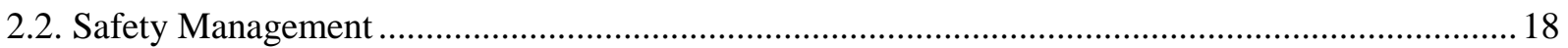

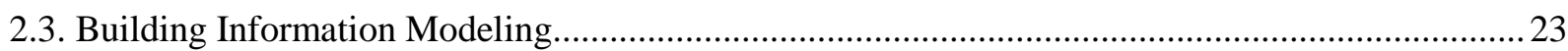

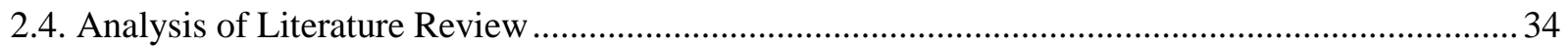

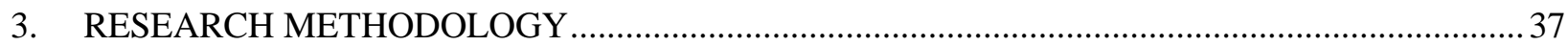

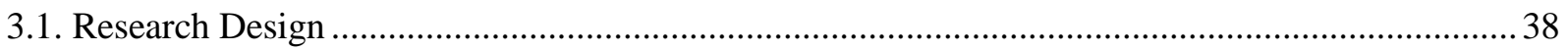

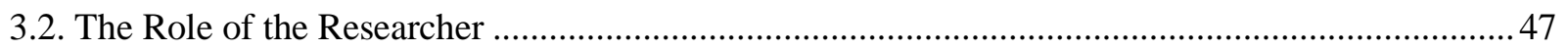

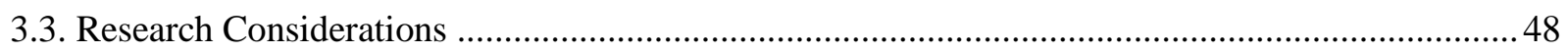

4. DATA COLLECTION, CATEGORIZATION, AND VALIDATION ...........................................51

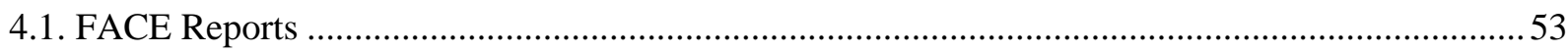

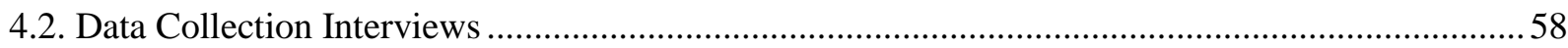

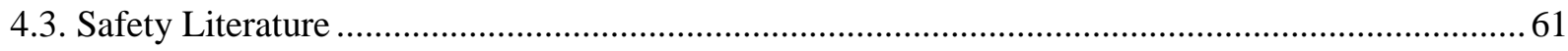

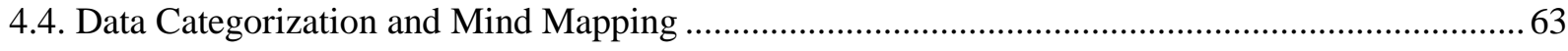

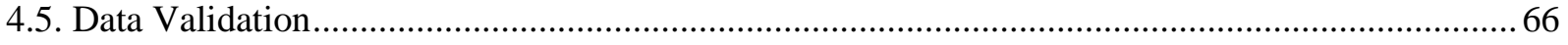

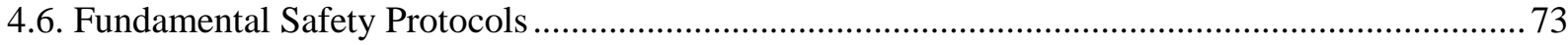

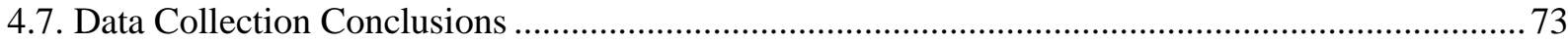

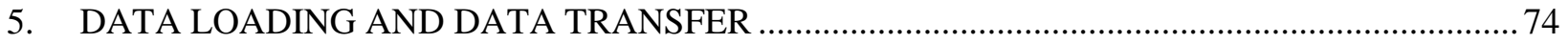

5.1. Phase I - Asset Safety Identification Tool (ASIT) ..................................................................... 75

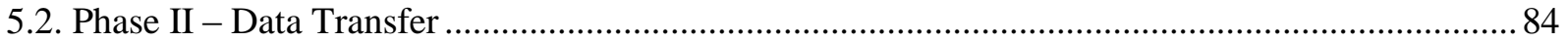

6. DATA RETRIEVAL AND PROCESSING SYSTEM (DRPS) ….............................................. 105

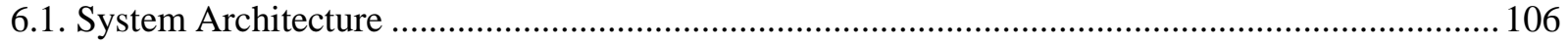

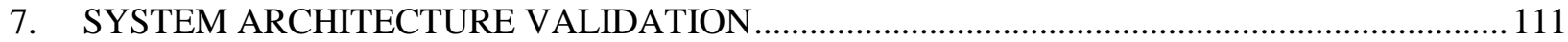

7.1. FM Test Case No. 1 - FACE Report 9013 ….......................................................................... 112

7.2. FM Test Case No. 2 - Coal Fired Boiler Preventative Maintenance ........................................... 115 


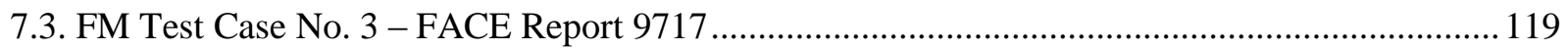

7.4. FM Test Case No. 4 - Liquid Anhydrous Ammonia \& Oil Separation ....................................... 123

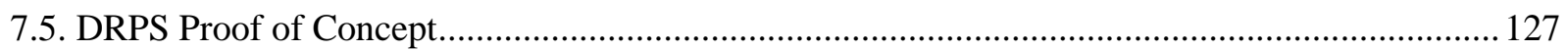

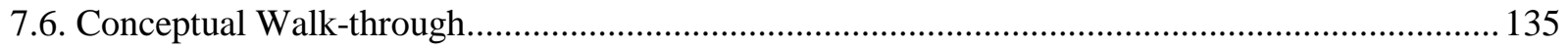

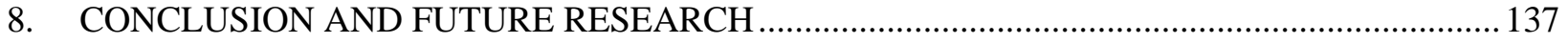

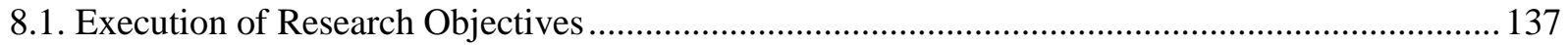

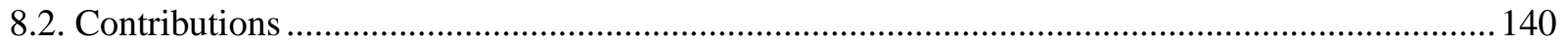

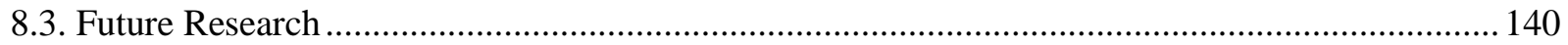

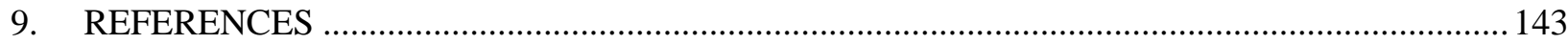

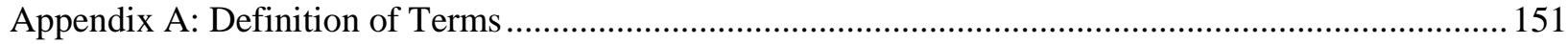

Appendix B: FACE Report Data Collection and Analysis Spreadsheets - Falls................................... 152

Appendix C: FACE Report Data Collection and Analysis Spreadsheets - Contact With / Struck By ..... 153

Appendix D: FACE Report Data Collection and Analysis Spreadsheets - Non Elec. H.E\&S................ 155

Appendix E: FACE Report Data Collection and Analysis Spreadsheets - Electrical H.E.\&S................ 156

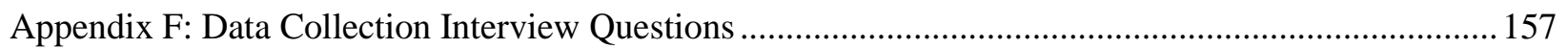

Appendix G: Data Collection Interview \& Analysis Spreadsheets - Sally ............................................ 159

Appendix H: Data Collection Interview \& Analysis Spreadsheets - Bill \& Chris ................................... 161

Appendix I: Data Collection Interview \& Analysis Spreadsheets - Joe, Dan, \& Tim ........................... 163

Appendix J: Safety Literature Data Collection and Analysis Spreadsheets............................................ 165

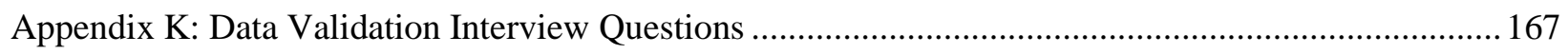

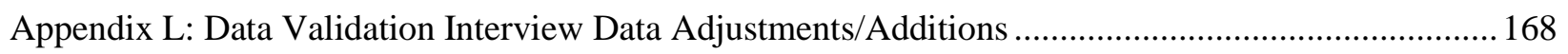

Appendix M: Safety Input Relational Information as Formulated in the ASIT.................................... 170 


\section{LIST OF FIGURES}

Figure 1: Comparison of Incidence Rates.............................................................................. 2

Figure 2: Applicable Safety Information Present in Multiple Sources............................................ 4

Figure 3: Safety Documentation Sources Occurring throughout the Facility Lifecycle................... 5

Figure 4: Safety Schema Development Methodology................................................................ 10

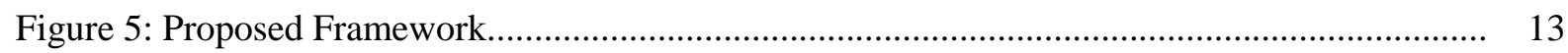

Figure 6: Literature Analysis Venn Diagram.......................................................................... 14

Figure 7: The Four Components of Safety Management ....................................................... 18

Figure 8: FM Fatalities by Year 2008-2012 _.......................................................................... 19

Figure 9: Event Information of FM Fatal Accidents 2008-2012 ................................................ 22

Figure 10: Event Information of FM Non-Fatal Accidents 2008-2012 ....................................... 22

Figure 11: Correlation of "Area of Research" and "BIM Technology" .......................................... 34

Figure 12: The DMADV Theoretical Framework in this Research............................................. 38

Figure 13: Research Methods within the DMADV Framework..................................................... 41

Figure 14: Phase I Data Collection Process................................................................................ 52

Figure 15: Evolution of the Main X-axis Nodes in the FACE Report Spreadsheet........................ 54

Figure 16: Image of the FACE Report Spreadsheet Evolution..................................................... 55

Figure 17: Coding Methodology of FACE Report - Example.................................................... 56

Figure 18: Coded Data Collection Interview and Organization - Example.................................. 60

Figure 19: Coded Safety Literature and Organization - Example................................................ 62

Figure 20: Initial Mind Map - Contact With / Struck By Example.............................................. 65

Figure 21: Mind Map - Harmful Environments and Substances................................................ 70

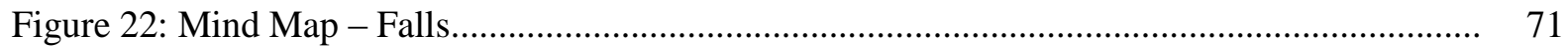

Figure 23: Mind Map - Contact With / Struck By............................................................. 72

Figure 24: Phase I and Phase II of the BIM-Based Safety Framework.......................................... 74

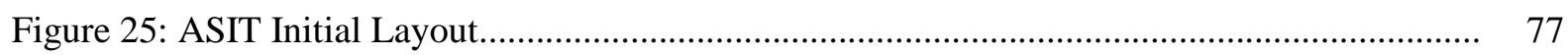

Figure 26: Dependent Inputs Requiring Values....................................................................... 79

Figure 27: Flow Diagram of ASIT Functionality ................................................................. 80

Figure 28: Longevity and Reusability of Information Forms and Formats.................................... 85

Figure 29: Data Transference Path for USC Case Study .............................................................. 89

Figure 30: Data Transference Path for MathWorks, Inc. Case Study ............................................ 91

Figure 31: Data Transference Path for BIMFMM Case Study ...................................................... 92 


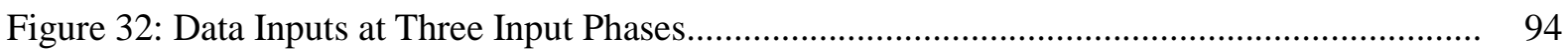

Figure 33: Data Transfer Mechanism within the Safety Framework.............................................. 96

Figure 34: Proof of Concept Model - Compressor Room............................................................... 97

Figure 35: "Native BIM-Model” Inputs in Navisworks Manage 2016.......................................... 98

Figure 36: User Defined Safety Property Using “Add New User Data Tab”.................................. 100

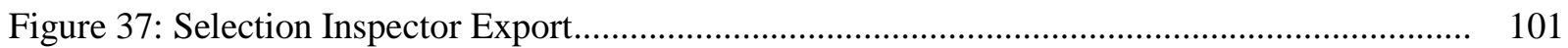

Figure 38: Exported Safety Information and Added "Environment Decibel Level (dBA)" Data..... 102

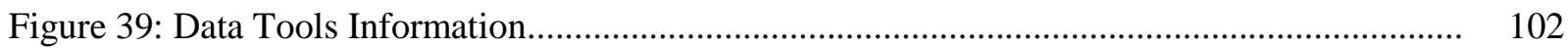

Figure 40: Added Safety Properties Under New "Safety Properties” Tab..................................... 104

Figure 41: Phase III of the BIM-Based Safety Framework............................................................ 105

Figure 42: DRPS Product Model............................................................................................. 107

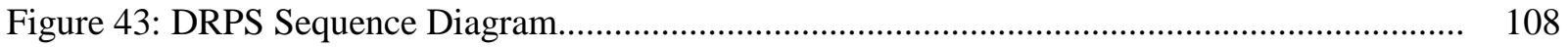

Figure 44: ASIT Data Load for Ducts -FM Test Case No. 1 1................................................... 113

Figure 45: Sequence Diagram for Ducts - FM Test Case No. 1 ................................................ 114

Figure 46: ASIT Data Load for Boiler1 - FM Test Case No. 2 .................................................. 116

Figure 47: Sequence Diagram for Boiler1 - FM Test Case No. 2 ............................................. 118

Figure 48: ASIT Data Load for PaperRewinder - FM Test Case No. 3....................................... 120

Figure 49: Sequence Diagram for PaperRewinder - FM Test Case No. 3 ................................... 122

Figure 50: ASIT Data Load for AmmoniaTank1 - FM Test Case No. 4........................................ 124

Figure 51: Sequence Diagram for AmmoniaTank1 - FM Test Case No. 4.................................. 126

Figure 52: Step 1 - Launch of the DRPS Plug-in....................................................................... 130

Figure 53: Step 2 - The DRPS Requesting the AssetName from the User..................................... 131

Figure 54: Step 3 - The DRPS Retrieving Safety Inputs and Values............................................ 132

Figure 55: Step 4 - The DRPS Evaluating the Returned Safety Inputs and Retrieving the AssetProtocols

Figure 56: Step 5 - SafetyInformation being Presented Upon Verification of the Retrieved AssetProtocols.

Figure 57: BIM-Based Safety Framework Processes by Phase. 


\section{LIST OF TABLES}

Table 1: Literature Review Resources by Category and Resource Type.................................. 15

Table 2: Planned vs. Unplanned O\&M Activity Examples........................................................ 16

Table 3: Pros and Cons of Safety Checklists ........................................................................... 20

Table 4: Available BIM Software by Category ............................................................................ 24

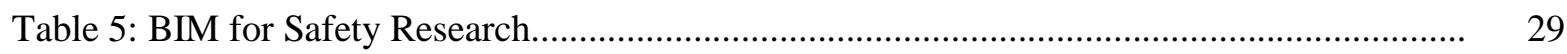

Table 6: Categorization of Area of Research and Percentage of Each Category......................... 33

Table 7: Categorization of BIM Technology and Percentage of Each Category.......................... 34

Table 8: Current Challenges This Research Attempts to Address............................................... 36

Table 9: Definition of the DMADV Methodology in the Research Context................................ 39

Table 10: Comparison of Terms for Quality and Rigor............................................................ 49

Table 11: Means of Trustworthiness in this Research................................................................ 49

Table 12: Breakdown of FACE Reports Utilized in this Research........................................... 53

Table 13: Safety Attributes within the Designated Category - FACE Reports........................... 57

Table 14: Data Collection Interview Participant Information.................................................. 58

Table 15: Safety Attributes within the Designated Category - Data Collection Interviews......... $\quad 61$

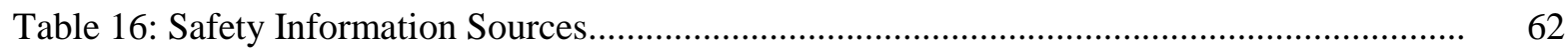

Table 17: Safety Attributes within the Designated Category - Safety Literature........................ 63

Table 18: Initial Categorization Safety Inputs, Data Sourcing, and Protocols (Partial)............... 64

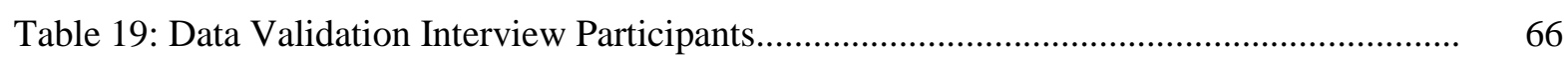

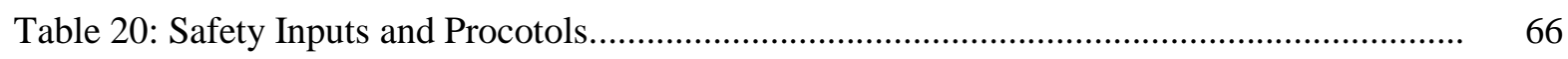

Table 21: ASIT Developed Safety Properties for Ducts .......................................................... 81

Table 22: ASIT Developed Safety Properties for IndustrialCFBoiler........................................ 81

Table 23: ASIT Developed Safety Properties for PaperRewinder.............................................. 82

Table 24: ASIT Developed Safety Properties for AmmoniaTank............................................. 82

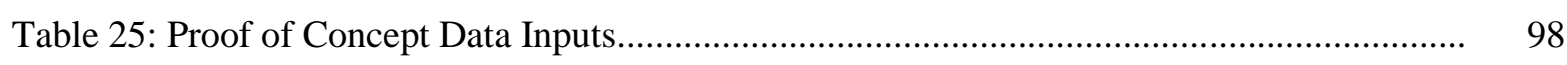

Table 26: Task Specific Queries Launched by the DRPS (Examples)...................................... 106

Table 27: Test Case No. 1 Relevant ASIT Data for ExteriorDucts............................................. 113

Table 28: Test Case No. 2 Relevant ASIT Data for Boiler1................................................... 117

Table 29: Test Case No. 3 Relevant ASIT Data for PaperRewinder........................................... 121

Table 30: Test Case No. 4 Relevant ASIT Data for AmmoniaTank1 ......................................... 125

Table 31: Conceptual Walk-through Participants.................................................................... 135 


\section{INTRODUCTION}

\subsection{Background}

Buildings in the United States and around the world are becoming increasingly complex, utilizing sophisticated technologies for communication and operational control. The role of facility management (FM) staff is critical to the planning, maintaining, and managing of these complex facilities (Global FM 2012). As skilled professionals, FM staff use knowledge in multiple disciplines such as electrical, mechanical, and plumbing to ensure the functionality of the built environment (International Facility Management Association 2013). Often, the complexity of the systems will dictate the requirements for FM staff and the expertise areas that are required for the management of the facility.

Due to the maintenance and repair requirements of these facilities, workers in this field are at high risk of injury including, electrical shock, falls, crushing, cuts, and bruises. As a result, FM workers in the United States have a much higher rate of injury and illness than the national average when compared to all other employment fields (See Figure 1) (Bureau of Labor Statistics 2014a). In the five years from 2008 through 2012, 293 people lost their life in the field of Facility Management, also referred to as General Maintenance and Repair (Bureau of Labor Statistics 2009a; Bureau of Labor Statistics 2010a; Bureau of Labor Statistics 2011a; Bureau of Labor Statistics 2012a; Bureau of Labor Statistics 2013a). This number accounted for roughly $1.3 \%$ of all occupational fatalities in the United States and has shown an increase of $64 \%$ from 2008 to 2012. In that same timeframe within the private sector, FM employers recorded 98,420 cases of occupational injuries and illness, with 26,190 cases requiring a minimum of 31 days away from work (Bureau of Labor Statistics 2009b; Bureau of Labor Statistics 2010b; Bureau of Labor Statistics 2011b; Bureau of Labor Statistics 2012b; Bureau of Labor Statistics 2013b). Case study analysis of the Fatality Assessment and Control Evaluation Program (FACE), issued by The National Institute of Occupational Safety and Health (NIOSH), confirms that many of the recorded accidents could have been avoided had the victim followed appropriate hazard mitigation information to safely execute the facility repair and maintenance (FRM) task.

To mitigate some of the risks associated with FRM tasks and to comply with many federal, state, and local laws, organizations provide job specific training and numerous safety specific documents to protect their FM employees (Occupational Safety and Health Administration 1998). These are proven and effective methods for the protection of staff, but require the information to be utilized comprehensively. In other words, no single training seminar or safety document supersedes the others. This requires the FM worker to comprehend all the safety information collectively and enact the applicable safety protocol with each 
FRM task. With the majority of onus on the worker's interaction with the safety information, it is not surprising that human error is the cause of $70-80 \%$ of all operational accidents (Leveson 2004).
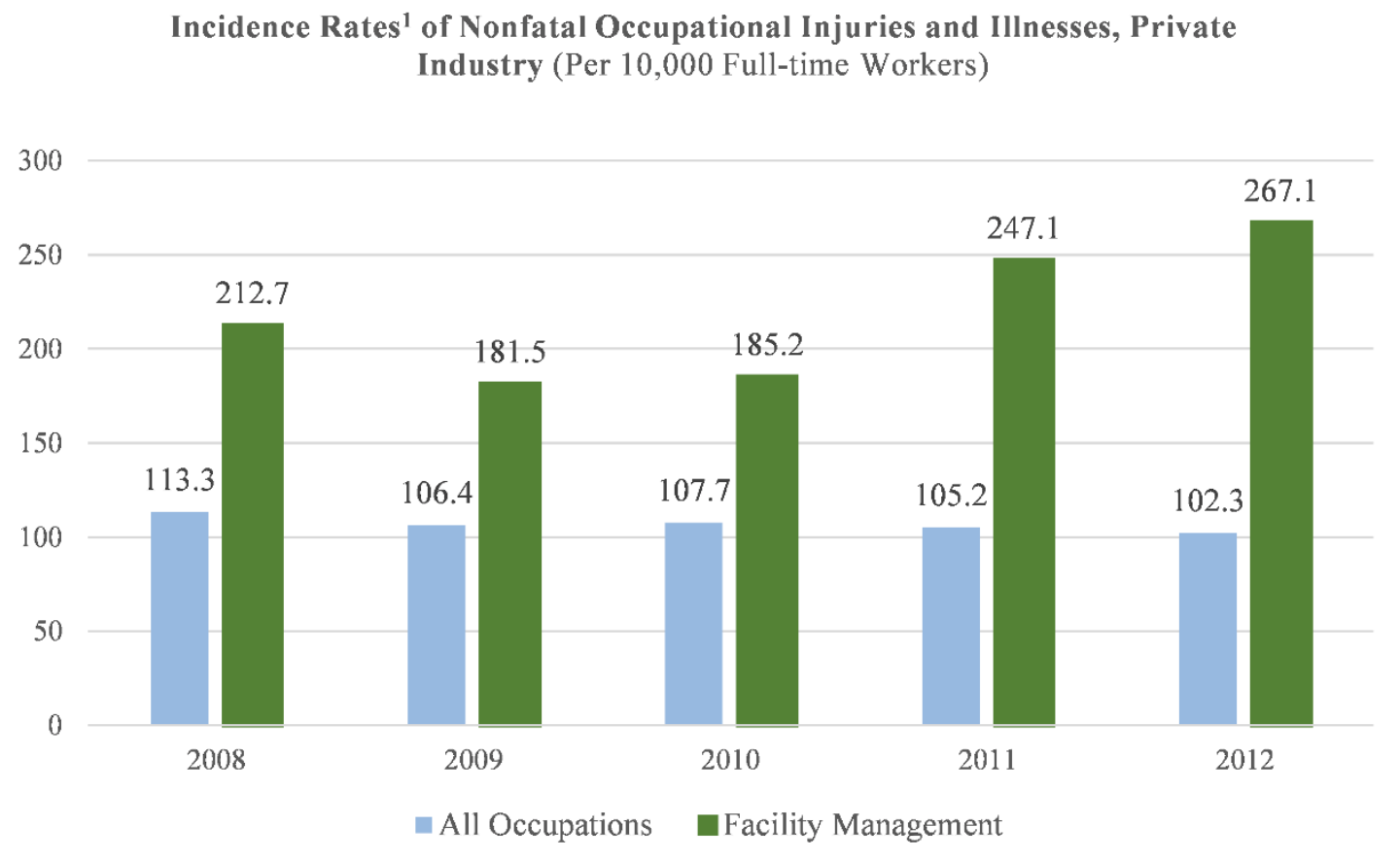

${ }^{1}$ Incidence rates represent the number of injuries and illnesses per 10000 full-time workers and were calculated as: $(\mathrm{N} / \mathrm{EH}) \mathrm{X} 20000000$ where $\mathrm{N}=$ number of injuries and illnesses $\mathrm{EH}=$ total hours worked by all employees during the calendar year $20000000=$ base for 10000 full-time equivalent workers (working 40 hours per week 50 weeks per year).

Figure 1: Comparison of Incidence Rates (Bureau of Labor Statistics 2009b; 2010b; 2011b; 2012b; 2013b)

Comprehensive safety information is often available within an organization; however, this information is often fragmented among multiple resources that would need to be referenced prior to a FRM work activity (Goedert and Meadati 2008; Lucas 2012). Research has shown that the more time and effort an individual must spend obtaining information, the less likely they are to retrieve the information and obey the stated warnings (Godfrey et al. 1985; Wogalter et al. 1989; Zeitlin 1994). Conversely, minimizing the amount of time and effort to the lowest possible level of information retrieval, has shown a much stronger likelihood of safety protocol implementation (Zeitlin 1994). This information retrieval is vital to the safety of facility management workers as daily tasks are often time sensitive and can require quick reaction in order to place the facility or critical equipment back into functioning condition. 
Within the field of FRM, there are two types of maintenance activities that can take place on a daily basis, reactive maintenance or preventative maintenance. Reactive, or breakdown maintenance, requires the replacement or repairing of faulty parts and/or components. This type of maintenance is estimated to account for roughly 34\% - 45\% of all maintenance activities (Blache 2010; Maintenance Assistant Inc. 2014). Reactive maintenance places a substantial burden on FM staff as a number of organizational disadvantages arise when this type of maintenance is necessary, including, direct and indirect costs of repair, equipment downtime, and interference with planned work. Additionally, the pressure to mitigate these disadvantages as quickly as possible creates safety issues by requiring staff to work under pressure to get the systems running without delay (Maintenance Assistant Inc. 2014).

Preventative or planned maintenance uses regular system and equipment checks on fully functioning assets or facilities to lessen the likelihood of failure. This type of maintenance activity is certainly more desirable to FM staff as preventative maintenance allows for planning of resources and strategy; however, to many organizations, this type of maintenance activity has also become a substantial issue. As with many industries, the financial crisis played a significant role in how FM staff managers and organizations allocated resources. During this time period, facility managers were tasked with cutting costs while keeping critical equipment online and functioning. The results of this mandate were staff reductions, limited funding, and deferred maintenance practices (Gager 2014; Harris and Rygielski 2014), creating a substantial backlog of maintenance needs.

Whether FM staff are reactively trying to repair a disabled asset or proactively maintaining a backlog of equipment needs, the requirement to complete work activities as quickly as possible is a daily occurrence. Working under the stress of too many work orders and short deadlines results in rushing, which has been shown to be directly correlated to occupational injuries and fatalities. According to The Lawrence Berkley National Laboratory (2012), "Injuries due to time pressure are most often the result of a conscious or semiconscious decision on the worker's part to circumvent a known preventative measure to a known safety hazard in the interest of getting the task done on time or rushing to keep ahead of a process following close behind." The inconvenience of having to retrieve uncategorized safety related information from a number of fragmented sources, retards the FRM task, requiring time sensitive activities to be rushed, and often distracting attention from hazards that would normally be recognized.

Exploring which contract entities input safety data, when the data are presented, where it is stored, and how it is extracted, provides insight into the fragmentation of current market safety protocol. This research explores a potential solution to mitigate the fragmentation of safety information, improving the reference 
time and required effort, by providing job specific safety protocols at the lowest possible level of information retrieval through the use of a singular BIM-based framework. The framework will act as an intermediary between the stored job specific safety protocols and the FM worker assigned to the task.

\subsection{Safety Information Sources}

Information that is applicable to the safe maintenance of a facility comes from a number of sources throughout a building's lifecycle. The information is often presented by the contract entities, through a number of contract required documents within a construction project. Figure 2 presents a number of examples of facility management applicable safety information and the typical source location of that information at the completion of a project, while Figure 3 presents at what point in the building's lifecycle that information is typically developed. To safely execute a facility management task, a FM worker may need to compile any number of the documents shown in Figure 2 to create a comprehensive safety protocol.

\section{Safety Documentation Sources}
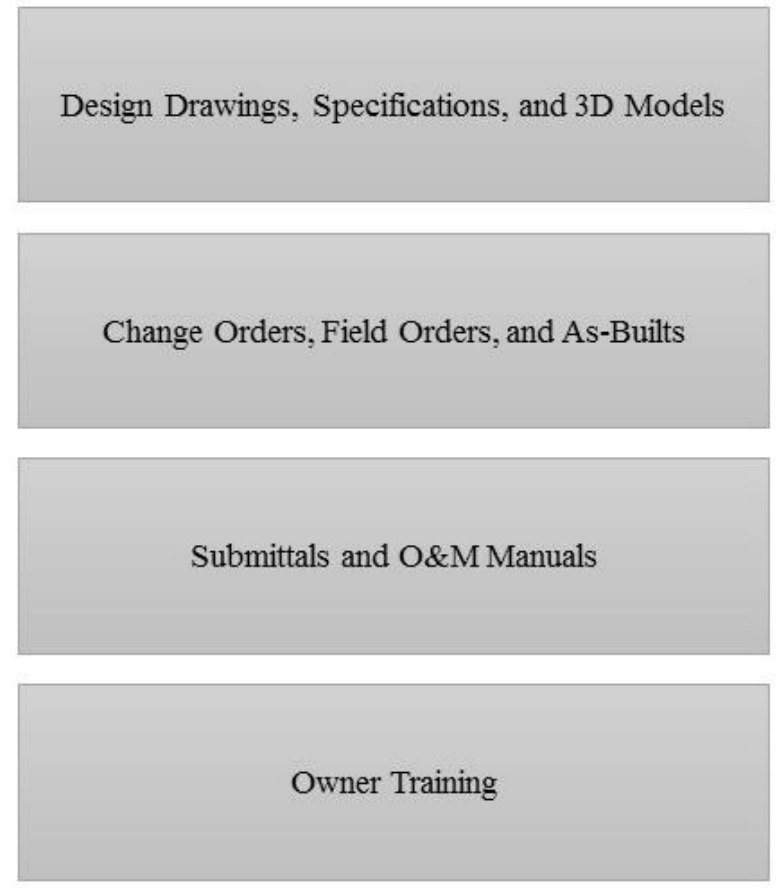

Prevention Plans, Safety Handbook, Safety Meetings, Safety Checklists, and Accident Investigation

\section{Applicable Safety} Information

- Power sources

- Disconnect locations

- Elevations

- Spatial planning

- DfS / PtD

Adjustments made to:

- Power sources

- Disconnect locations

- Elevations

- Spatial planning

Equipment maintenance procedures

- Testing curves and requirements

- Manufacturer's information

- Warranty Information

Equipment function

- Maintenance process

- Video or meeting minutes from training seminar

- PPE Requirements

- User manuals (e.g. forklift)

- Tool safety

- Internal policies and procedures

Figure 2: Applicable Safety Information Present in Multiple Sources 


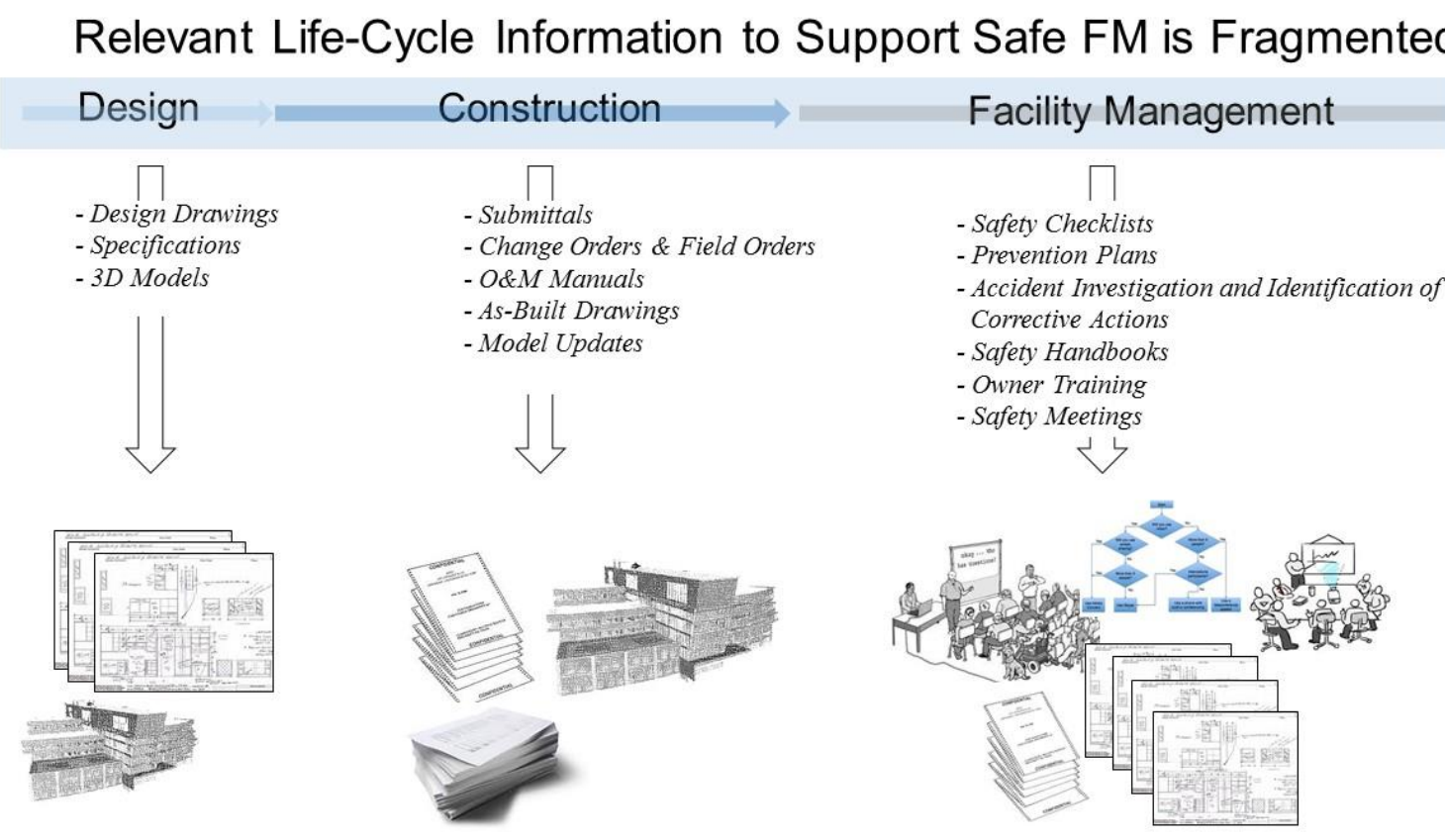

Figure 3: Safety Documentation Sources Occurring throughout the Facility Lifecycle

When architects and/or engineers (A\&E) begin to design a building, a number of safety decisions are being made. The routing of power, proximity of disconnects, the number of isolation valves, the elevation of equipment components, and many other considerations, all affect the maintenance requirements during the facility management phase. A conscious understanding of this cause and effect and the subsequent design in support of downstream lifecycle phases is known as Prevention through Design (PtD) or Design for Safety (DfS) (Gambatese et al. 2008; Ku and Mills 2010; Prevention through Design: Design for Construction Safety 2014). The use of PtD/DfS is a powerful tool to improve accident mitigation; however, has historically been focused on the construction phase and less on facility management.

By using PtD/DfS for the FM phase, some hazards can be mitigated (e.g. using a chain wheel for valves at high elevation). Other safety hazards are inherent to the work activities or cannot be mitigated due to site restrictions, process restrictions, congestion, and the like. These hazards will be present despite any design considerations (e.g. electrocution risk associated with high voltage gear). Regardless of the nature of the hazard, the safety concerns/considerations that are made by the A\&E will impact the maintenance requirements of the facility. 
Along with the considerations made by the design team, the capturing of supplier/contractor procurement decisions within a project could also play a significant role in the development of FM safety protocols. Contractor selection of a manufacturer for procurement of materials and/or equipment results is a substantial amount of applicable safety information that is presented through submittals and O\&M manuals. Information such as maintenance cycles, maintenance protocol, required tools, and contact information, all play a role in the downstream development of a safety protocol. Recently, with a focus on BIM-FM, FM personnel have become involved in projects during the design and construction phase in order to aid in this type of decision making. This is often achieved through specific equipment specifications or collaborations with suppliers/contractors.

In addition to project specific information, safety information applicable to the facility management staff will come from organizational policies and procedures. Through safety meetings, literature, manuals, and legal precedence, the internal requirements for the maintenance of a facility plays an integral part in the development of safety protocol. As the employer of facility management staff, the owner/facility manager is ultimately responsible for the safe maintenance of the facility. Failure to properly educate staff or maintain a safe working environment could result in worker's compensation claims and/or litigation (Commonwealth of Massachusetts 2014).

\subsection{Fragmentation of Relevant Safety Information to Support Facility Management}

Research has shown that the more time it takes a person to obtain safety information, the less likely that individual is to reference or retrieve the information and obey the stated warnings (Godfrey et al. 1985; Wogalter et al. 1989; Zeitlin 1994). In facility management, safety information is fragmented among multiple resources, developed at various points in a project's lifecycle, creating inefficiencies in the procurement of information as shown in Figure 2 and Figure 3. When a piece of equipment requires maintenance or repair, the FM worker will need to address all of the safety concerns that are applicable to that equipment. This will likely require referencing multiple documents to identify comprehensive safety concerns surrounding the task. The inconvenience of having comprehensive safety information scattered through multiple documents, coupled with the often present time sensitivity inherent to FM tasks, can result in FM personnel bypassing the retrieval of applicable information, exacerbating the likelihood of workrelated fatality, injury, or illness.

Recently, a significant push has been made to incorporate operations and maintenance information into BIM models or computer maintenance management systems (CMMS) in order to consolidate the information to improve efficiency in retrieving information. Through the use of BIM interoperability, 
virtual databases, and add-ons such as COBie, an improvement in O\&M storage and retrieval has been achieved. However, this information has not focused on the storage and retrieval of safety information, and although there may be a small percentage of applicable information overlap, safety related information often remains unorganized and uncategorized. Nevertheless, utilizing these existing systems for storage and retrieval of safety information remains a viable and promising avenue of research. As shown by Meadati \& Irizarry (Meadati and Irizarry 2010), building information models can act as a single, centralized database for knowledge storage and retrieval. The use of interoperability, virtual databases, and COBie are explored in much greater detail in subsequent chapters.

The following example presents a typical FRM task that a worker may encounter at a large utility or industrial plant. This example is intended to present the applicable safety information required for the activity and where that information is typically stored.

\section{Project Example}

A facility management worker receives an annual maintenance request to assess a Motor Control Center (MCC). An MCC is an assembly of combination starters in a single enclosure that contains motor starters, fuse or circuit breakers, and a disconnect (Siemens Technical Education Program 2014). These are commonly found in commercial or industrial applications where a number of motors are present and the owner wishes to consolidate the motor controls into one housing.

In this scenario, the FM worker will need to review the design drawings/specifications, 3D model, and/or CMMS system to find information such as power source, components, disconnect location, and schematics. This will provide a "lay of the land" and allow the FM worker to prepare for the MCC prior to opening the cabinet. For information such as MCC maintenance protocol, safety precautions (such as arc flash), warranties, and manufacturer information, the worker will need to review the O\&M Manual provided by the contractor/manufacturer. Information within the $O \& M$ manual will often incorporate operations as well as some equipment specific safety information. Finally, the worker must abide by the high voltage gear safety protocol established by their organization. This information is typically available in a number of safety manuals, meeting minutes, OSHA documents, or adopted safety literature. All of this information will need to be extracted in order to develop of a comprehensive safety protocol that must be enacted by the FM worker to maintain a safe working environment.

Although some of this information may be stored in a virtual database or 3D model, it is unlikely that comprehensive, job specific safety related information would be available. By identifying and consolidating 
the safety information relevant to the FRM task, the convenience in accessing and obtaining comprehensive safety protocol for the MCC is greatly improved. Minimizing the FM worker's inconvenience and providing a singular point for interaction would result in a greater likelihood of reference and safety protocol execution.

\subsection{Statement of Problem}

The following statements represent the problems that this research addresses.

Problem No. 1: Applicable project related safety information is often available at the handover stage from construction to facility management, but is not appropriately identified for facility management tasks. The information that would provide relevant safety data for a specific work activity is often uncategorized and therefore is left at the discretion of the worker whether or not the information should be utilized for a given task.

Problem No. 2: Fragmentation of applicable safety information within multiple project documents (O\&M manuals, plans, specs, SDS, etc.), incomplete virtual databases, and/or multiple file formats creates inconvenience in obtaining comprehensive information, reducing the likelihood of reference by FM staff. This has been shown to impact hazard mitigation and increase the probability of work related fatalities, injuries, and illnesses.

\subsection{Research Goal and Objectives}

The goal of this research is to mitigate the fragmentation and timing inefficiencies within safety related information retrieval by developing a BIM-based framework to categorize, standardize, consolidate, and add logic to asset specific safety information. This is executed by establishing what safety information is important to FRM tasks, how the data is transferred, and how logic can be added to the safety data in order to efficiently present the information. By leveraging much of the work executed to date in the Building Information Modeling-Facility Management (BIM-FM) field, established and tested methods of data storage and transference can be utilized in order to develop a BIM-based framework.

By organizing the applicable safety data, patterns or commonalities are exposed. These patterns of data allow for the development of a set of inputs (known as "codes" in qualitative research) that can be associated with specific work activities that are relevant to the three safety hazards that this research is exploring. Using a Six Sigma Methodology to collect and analyze the data and validation methods using industry professionals, this study maintains quality and rigor commensurate with high level research. 
Once the safety data is identified, the information can be placed into the framework. The structured inputs and associated values are placed into a comprehensive Navisworks model through a data transference mechanism. Through the use of existing software, the safety data can interact with a Data Retrieval and Processing System (DRPS). The DRPS utilizes rules to deliver task specific safety protocols based on stored asset information (safety values) and user input responses. This interaction between the FM worker and the DRPS, is presented through a UML Class Diagram, a Sequence Diagram, and a Conceptual Graphical User Interface (GUI). Use of this framework prior to the execution of a work activity will increase the likelihood of obtaining comprehensive safety information efficiently, better preparing the FM worker for the impending task. This will aid in the development of a safety plan that is specific to the equipment requiring maintenance or repair. The attainment of this goal will be carried out by the execution of the following objectives:

Objective No. 1 - Define, categorize, and standardize asset specific safety information applicable to falls, contact with / struck by, and harmful environments \& substances.

The research uses qualitative coding to categorize safety information and applicable attributes within the three safety categories this research addresses. This is executed to develop a safety inputs and associated values as shown in Figure 4. To complete this objective and to mitigate the issue identified in Problem Statement No. 1, the following design is utilized:

- Develop a comprehensive list of safety inputs classified within the scope of this research

- A thorough review of safety documentation, NIOSH FACE reports, and interviews (Data Collection) are utilized in order to obtain a comprehensive list of safety related properties (inputs and values) relevant to FM workers.

- Data validation interviews are utilized to validate the safety related properties with industry experts.

- Organize, categorize, and standardize safety related information.

$\circ$ Once identified, the safety related information is organized and categorized using spreadsheets \& mind-mapping.

- Organized and categorized information allows for the development of an approach to standardize asset specific safety properties that can be input into a comprehensive BIM Model. Defining these properties and identifying which contract entity is responsible for supplying the values for each property allows for the applicable safety related information to be input into the system. 


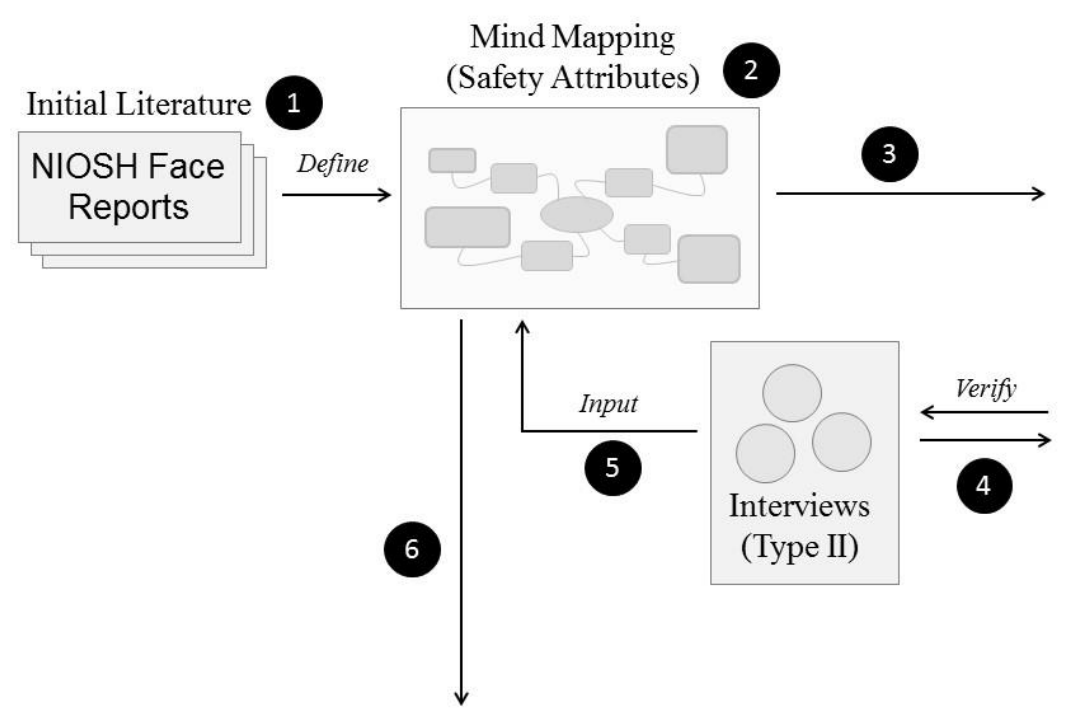

Finalized Safety Properties
Additional Literature Review

NIOSH Face Reports

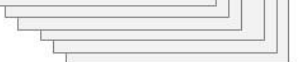

Interviews (Type I) with Industry Experts

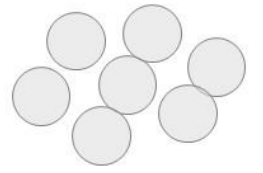

Safety Literature Review

- O\&Ms

- Handbooks

- OSHA

- Specs

Figure 4: Safety Schema Development Methodology

The initial literature review of the NIOSH FACE Reports validates that safety information can be coded and placed into a mind map (2). Upon verification, an additional literature review (3), utilizing FACE reports, interviews (data collection), and safety literature takes place to obtain a more comprehensive review of the safety attributes and values associated with the categories this research is addressing. (4) At this point a second round of interviews (data validation) take place to validate the information gathered from the FACE reports, interviews (data collection) and safety literature. Once validated, the safety related information is organized and categorized using spreadsheets \& mind-mapping (5) and produce finalized, coded, safety properties (6).

Objective No. 2 - Present a data path through defined transfer mechanisms in order to get safety information, in various formats, from design/construction to FM personnel into a singular repository.

- Categorized safety attributes and values are processed through various mechanisms, based on the applicable contract entities' existing infrastructure. The relevant safety information may come in the form of interoperable models, non-compatible models, documents, and other formats.

- In order to place all of this information into a comprehensive model, a number of IT Tools for data management and model integration are utilized. 
- Once comprehensive relevant safety information is available within the BIM model, the information is available to interact with the developed Data Retrieval and Processing System (DRPS).

Objective No. 3 - Develop a data retrieval and processing system (DRPS) and conceptual graphical user interface (GUI), presented through graphics, to represent the interface between FM worker and DRPS.

- A data retrieval and processing system (DRPS) is used to add logic and guide the information exchange in order to provide applicable and necessary information to FM staff in a timely manner. For this research, structural and behavioral diagraming is utilized in the form of UML Class Diagrams and Sequence diagrams to capture the details of the static classes and relationships between the classes. The DRPS uses test cases in order to validate the functionality of the developed Class and Sequence diagrams.

- Through Java Eclipse, develop graphics for visual presentation of the conceptual GUI.

- Utilize the GUI in correlation with the Sequence diagrams to present a Proof of Concept.

Objective No. 3 is executed to mitigate the issues identified in Problem Statement No. 2.

Objective No. 4 - Validation

- Use professionals in the field of facility management to validate safety properties, data categorization, and framework through a cognitive walkthrough.

\subsection{Research Assumptions and Limitations}

The following section presents the assumptions and limitations made within the scope of this research.

Assumption No. 1 - Organizations wishing to utilize this data framework will have the appropriate infrastructure to run building information models.

To utilize the framework presented in this research, a facility management department must have the expertise, hardware, and software to access the building information model. This research assumes that any user implementing the framework presented herein will have these capabilities. If these capabilities are not readily available, additional infrastructure setup will be required by the implementing entity.

Assumption No. 2 - Users interacting with the system have the knowledge of BIM software and application. 
Much of the system architecture of this research is built around the premise of BIM-FM, the use of a building information model during the facilities management phase. Users interacting with the DRPS will need to be comfortable working with data loaded graphical models.

Limitation No. 1 - This research will not address all possible hazards that facility management staff encounter.

This research will focus on the development of safety protocols for three of the major safety categories. These categories are as follows:

- Falls - Injuries and fatalities resulting from falls to lower levels.

- Struck-by/contact-with - Injuries and fatalities resulting from being hit with or forced against an item or equipment causing a crushing or slicing. This incident type is often related to automated equipment systems.

- Exposure to harmful substances/environments - Injuries and fatalities resulting from an environment that contains hazardous chemicals, toxic air, a lack of oxygen, or electrified equipment or surroundings. This category will focus mainly on confined spaces, electrified equipment, and hazardous environments.

Other types of accidents will not be addressed within this research. The selection of these three hazard types is explained in greater detail in Section 2.2.1.1 Research Defined Hazard Types.

Limitation No. 2 - The role of human interaction and safety culture in worker safety will not be addressed in detail.

This research recognizes the importance of the human interaction within safety scenarios, but will not address this aspect. In addition, the impact that safety culture has on the implementation of safety protocol and the FM worker's desire to enact the appropriate steps to mitigate risk are not addressed.

\subsection{Proposed Framework and Research Contributions}

Figure 5 presents the complete framework for the research. Throughout the lifecycle of the project, relevant safety inputs are given values by the various contract entities responsible for those assets from what would otherwise be fragmented safety information. Through various data transfer mechanisms, based on the type and format of the safety submissions, the relevant safety information is placed into a data storage repository. Utilizing process flows and existing IT tools for data management, such as software interoperability, Selection Inspector, and DataTools, the correct information can be stored in a similar format. In addition, 
relevant safety information provided by the owner of the facility can be input into the system to meet organization specific requirements.

In order to retrieve task specific safety information, the user will launch the DRPS and select the asset requiring maintenance. Based on the values already in place for that particular asset, two background activities will take place. First, relevant safety applicable information will be sent to the end user interface for finalized safety protocol reference. Second, the DRPS will identify the need to ask the user a series of short questions in order to develop a task specific protocol based on the asset being serviced, environment, and circumstance. Utilizing a question based system urges the worker to proactively consider the safety plan prior to the execution of the task, similar to the tactics used in inquiry-based learning. Based on the asset selected and the responses by the user, a safety protocol output will be delivered to the FM worker.

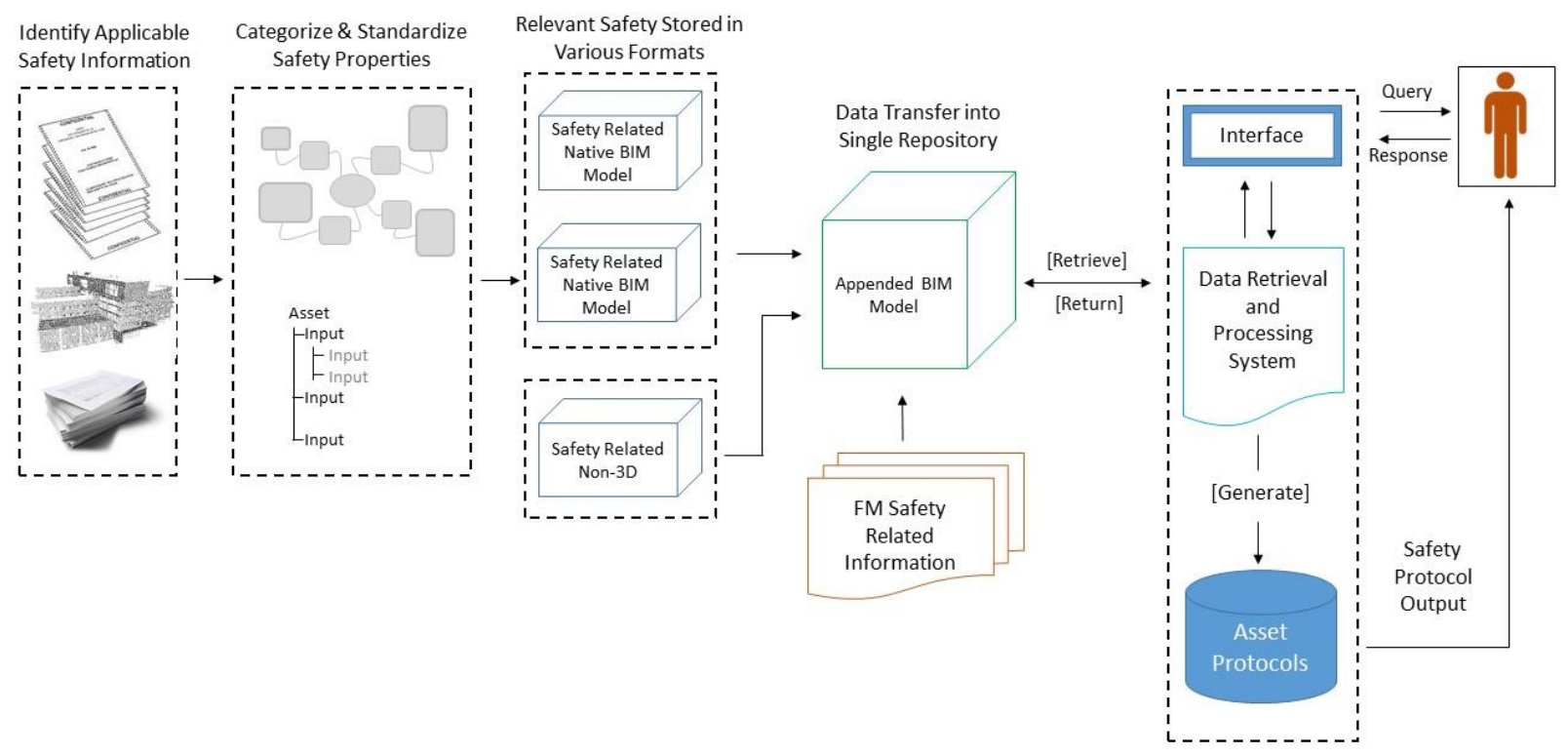

Figure 5: Proposed Framework

The proposed framework attempts to support safety during the facility management phase through two research contributions. These contributions are as follows:

- Develop comprehensive safety information in an effort to mitigate incidents in falls, contact with/struck by, and harmful environments \& substances.

- Developed an approach to identifying asset group specific safety properties. 


\section{LITERATURE REVIEW}

This chapter utilizes a literature review and gap analysis to identify current market trends and available technologies to support the current need for facilitating necessary information to perform facility management operations in a safer environment. In addition, a new method for facility management workers to interface with applicable safety protocol is proposed.

By exploring the topics of safety during facility management, the use of BIM for facility management, and the use of BIM for safety, a gap was identified in the form of the incorporation of BIM for safety during facility management as shown in Figure 6. These detailed research concepts were developed from three core concepts of facility management, safety management and building information modeling. A thorough understanding of the core concepts and sub-concepts of this research is necessary to identify the current processes utilized during facility management activities.

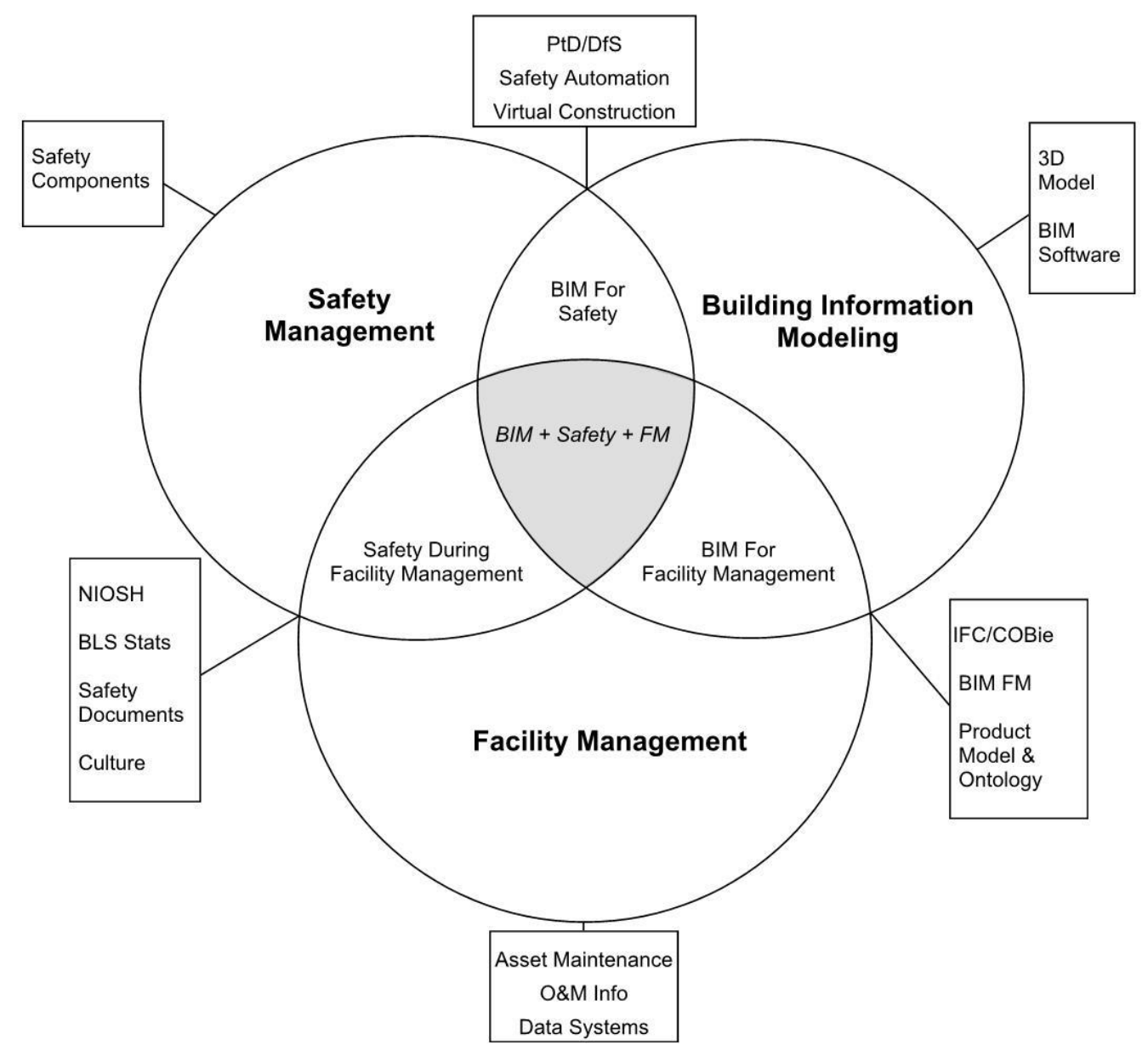

Figure 6: Literature Analysis Venn Diagram 
In order to obtain a detailed understanding of current trends and research, this literature review utilized online resources such as OSHA, facility management, computer engineering, government websites, and peer-reviewed journal articles on the various topics. Additionally, discussions with industry experts were executed to provide a general understanding of topics. Table 1 presents the resources utilized in each section of the literature review. The study will critically analyze the three areas stated and then comprehensively review and analyze the intersection of these three areas.

Table 1: Literature Review Resources by Category and Resource Type

\begin{tabular}{|l|l|l|}
\hline \multicolumn{1}{|c}{$\begin{array}{c}\text { Safety During Facilities } \\
\text { Management }\end{array}$} & BIM for Facility Management & \multicolumn{1}{c|}{ BIM for Safety } \\
\hline Online Resources (14) & Online Resources (5) & Online Resources (3) \\
\hline Journal Articles (6) & Journal Articles (21), Book & Journal Articles (22) \\
\hline $\begin{array}{l}\text { Industry Expert Discussions (4) } \\
\text { Included: Engineering Firm, } \\
\begin{array}{l}\text { Major Utility Owner, University } \\
\text { FM Staff, Facility Safety Expert }\end{array}\end{array}$ & $\begin{array}{l}\text { Industry Expert Discussions (4) } \\
\text { Included: BIM Software } \\
\text { Developer/User, BIM FM } \\
\text { Consultants (3) }\end{array}$ & $\begin{array}{l}\text { Industry Expert Discussions } \\
\text { (111th Congress of the United } \\
\text { States of America) } \\
\text { Included: Professor (Safety } \\
\text { Expert) }\end{array}$ \\
\hline
\end{tabular}

Exploration of safety during facility management, the use of BIM for facility management, and the use of BIM for safety provided a comprehensive understanding of current market trends and research. A comprehensive, critical analysis of these areas as individual sections, allowed the research to identify overlap, eventually exposing a research potential. The proposed research framework will address this potential and present a solution to fragmentation and safety concerns within FM by incorporating BIM + Facility Management + Safety. Figure 6 graphically presents the literature review approach that this study utilized to identify the research potential. Utilizing the information obtained through existing literature and current market trends aids in the development of the applicable safety protocols and proposed research framework.

\subsection{Facility Management}

Facility management is a profession that encompasses multiple disciplines such as electrical, mechanical and plumbing, to ensure the functionality of the built environment by integrating people, place, process and technology (International Facility Management Association 2013). Facilities management can be executed through in-house staff or by a third-party contract (FacilitiesNet 2014). The caretaking of a building is crucial to maximizing the service life of the building and equipment; however, this optimization does not come without a price. After payroll, facility management costs are typically the greatest administrative expense to an organization (Cotts et al. 2010). Failure to appropriately maintain a facility can adversely 
impact the performance of an organization through equipment failure, the health of the organization's staff, and the safety of the building occupants. Conversely, a well maintained facility can enhance an organization's performance by contributing to the optimization of the working and business environment (Alsyouf 2007; Atkin and Brooks 2000; Roelofsen 2002).

The facility management department in any given organization may have many roles. From asset evaluation to design and construction consultation, the FM department needs to be aware of the organization's current assets and any planned additions, renovations, or new construction. In most cases, the largest role of the FM department is operations and maintenance (Cotts et al. 2010), usually identified simply as O\&M. O\&M is defined as "the activities related to the performance or routine, preventative, predictive, scheduled, and unscheduled actions aimed at preventing equipment failure or decline with the goal of increasing efficiency, reliability, and safety" (Environmental Protection Agency 2013). There are two types of O\&M activities, planned and unplanned. Planned activities are defined as, "programmed, preventative and cyclical," while unplanned activities are "reactive" (Booty 2009). Examples of planned and unplanned O\&M activities are identified in Table 2:

Table 2: Planned vs. Unplanned O\&M Activity Examples

Planned O\&M Activity (Preventative)

Air conditioning unit filter change

Millivolt drop test at circuit breaker

Water heater tank flush

\section{Unplanned O\&M Activity (Reactive)}

Air conditioning condenser leak repair

Replacement of corroded contacts

Replacement of pressure relief valves

Regardless of whether an O\&M activity is planned or unplanned, the facility management staff is responsible for the caretaking of the facility. These individuals are highly skilled in a number of crafts that allow for versatility in maintenance scope. According to the Bureau of Labor Statistics (2013c) the field of facility management employed 1,230,270 people in 2012. Projections for this profession show a 9\% growth through the year 2022 (Bureau of Labor Statistics 2014b). As the profession continues to grow and the complexity of buildings continues to increase, construction technology like BIM can provide benefit to the lifecycle of a facility and the safety of the staff (Teicholz 2013). 


\subsubsection{Facility Management Data Systems}

To aid FM departments in maintaining and tracking assets, issuing work orders, and executing a number of other FM functions, software is available to track these data systems. Facility management and operations staff work with a variety of tools ranging from manual paper and spreadsheets, to more advanced computer based systems including Computerized Maintenance Management Systems (CMMS's), Computer-Aided Facility Management (TabsCAFM) tools, and Building Management Systems (BMS) (Parsanezhad and Dimyadi 2014).

Computerized Maintenance Management Systems (CMMS) are utilized by facilities maintenance organizations to record, manage and communicate their day-to-day operations (Sapp 2015). CMMS can be deployed for asset management, inventory control, generation of service requests, managing work orders of different types, and tracking the resources (time and costs) of services and materials used to complete work orders (Parsanezhad and Dimyadi 2014; Teicholz 2013). FAMIS (by Accruent), IBM Maximo, Corrigo, WebTMA (by TMA Systems), and AiM Maintenance Management (by AssetWorks), Vizelia, Ryhti and Rambyg are some examples of CMMS's (Mitchell and Schevers 2005; Parsanezhad and Dimyadi 2014). Many of these systems supports integration of BIM and IFC.

Computer-Aided Facility Management systems integrates a Computer-Aided Design (CAD) graphics module and a relational database software to provide various Facility Management capabilities (Sapp 2015) including space management tools (e.g. administering room numbers, departments, usable heights, room areas etc.). CAFM systems also provide means to collect data from a variety of sources through technology interfaces to other systems (such as CMMS) or human transfer processes. Many CAFM systems are webbased. FM:Interact (by FM:Systems), Archibus, and AiM Space and Facilities Management (by AssetWorks) are some examples (Parsanezhad and Dimyadi 2014; Teicholz 2013).

Building Automation Systems (BAS) are centralized, interlinked, networks of hardware and software, which monitor and control the facility environment to ensure the operational performance of the facility as well as the comfort and safety of building occupants (KMIC Controls Inc. 2015). Most of the automation system is behind the scenes as hardware devices mounted to equipment or hidden underfloor or in the ceiling. Some personalized control can be made available through thermostat-like devices. From a central management perspective, the BAS resides as software on an operator's computer or is available as a web page. 
Even with the wide variety of software applications available to service facilities management needs, there is no single application that would encompass the diversity of all FM requirements (Sabol 2008).

\subsection{Safety Management}

The Federal Aviation Administration (2010) defines safety management as a, "formal, top-down business approach to managing safety risk, which includes a systemic approach to managing safety, including the necessary organizational structures, accountabilities, policies and procedures." The FAA recognizes four components to safety management (Federal Aviation Administration 2009):

- Safety Policy

- Safety Assurance

- Safety Risk Management

- Safety Promotion

Each of these components is described in greater detail in Figure 7. The example presented by the FAA is an exemplar model for occupational safety, including safety during facility management.

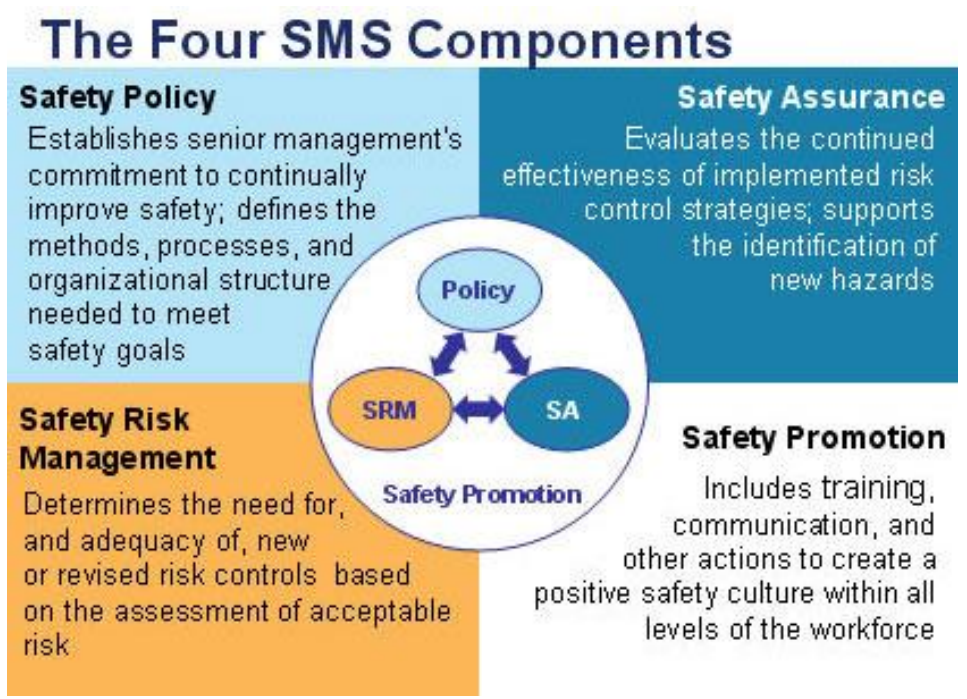

Figure 7: The Four Components of Safety Management (Federal Aviation Administration 2009)

\subsubsection{Safety During Facility Management}

This section addresses the current market trends and factors that are applicable to safety during facility management. Review of literature developed by large public and private entities and a governing safety 
body (OSHA), presents a snapshot of the industry as it stands today. Obtaining an understanding of the approach being utilized by FM staff provides an insight into the factors responsible for the high injury and illness rates and increasing fatality rates (Figure 8) present within facility management.

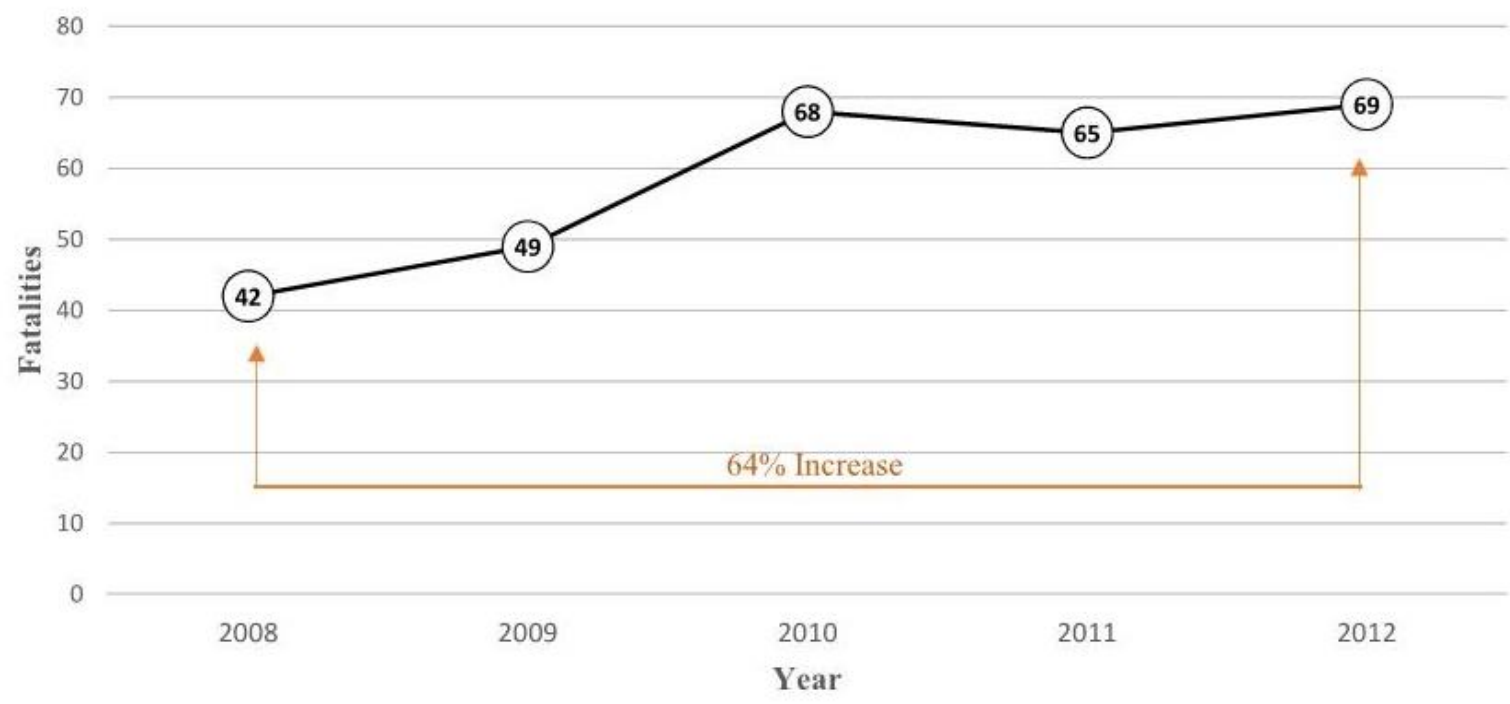

Figure 8: FM Fatalities by Year 2008-2012 (Bureau of Labor Statistics 2009a; 2010a; 2011a; 2012a)

For facility management staff, a thorough understanding of potential health risks that may be encountered during a work activity and the safety protocol utilized to mitigate the risks is of paramount importance. To remain proactive, many organizations maintain injury and illness prevention programs to reduce occupational injuries, illnesses, and fatalities (Occupational Safety and Health Administration 2013). These documents, although good for general safety information, are not tailored to specific work activities that a facility management worker would encounter on a daily basis. Legally, an organization is required to, "satisfy the ever-increasing number of federal, state, and local statutes relating to institutional health, safety, and the environment" (The University of Vermont Risk Management Group and Sarah Forbes Creative 2004). To comply with these requirements, many organizations develop risk management or safety handbooks specifically for the facility management department. These handbooks are developed to provide general safety guidelines for facility management staff including information on forklifts, personal protective equipment, rigging, scaffolding, and the like (e.g., Creighton University Facilities Management 2010). 
In addition to safety handbooks and prevention programs, many facility management departments will hold, daily, weekly, or monthly safety meetings to discuss safety concerns, incidents, accidents and information on safety topics (University of Maryland Facilities Management 2012). Similar to safety handbooks, these meetings are an effective method for conveying general safety topics or reactively discussing an incident, but are often executed in a lecture format that has been shown to be less effective than an active learning approach. Safety meetings, also known as "toolbox talks" or "safety talks," typically present OSHA safety instruction, jobsite hazard training and general safety awareness for items like ladder safety, eye protection, and working at elevation (Duke Facilities Management 2014; Facilities and Services 2014).

To address specific work activities, some organizations have developed checklists that are reviewed prior to the initiation of an FM task (City of DuPont 2010). Checklists can be an effective tool if utilized correctly; however, there are a few inherent issues with relying solely on safety checklists. Table $\mathbf{3}$ evaluates the pros and cons of utilizing safety checklists.

Table 3: Pros and Cons of Safety Checklists - Adapted from (Toups 2004)

\begin{tabular}{|l|l|}
\hline \multicolumn{1}{|c|}{ Pros of Safety Checklists } & \multicolumn{1}{c|}{ Cons of Safety Checklists } \\
\hline Simple form of hazard analysis & May be irrelevant for complex equipment \\
\hline Easy to use & Limited to expertise of its author(s) \\
\hline Quick results, allows work to get underway & Hazard identification is subjective \\
\hline
\end{tabular}

Safety checklists can be a valuable tool, but as a standalone document rarely include enough information to fully encompass the dangers associated with specific job activities (Toups 2004). Regardless of the work activity a facility management worker is executing, the individual should have a working knowledge of the organizational, departmental, and individual safety requirements identified by that worker's company.

In addition to documentation, facility management departments typically require new and existing employees to attend training seminars. Training is an essential part of implementing accident prevention and gives a strong foundation for general safety processes and requirements (City of DuPont 2010). Training may be required to comply with federal, state or local law. Federally, the Occupational Safety and Health Act of 1970 was developed, "to assure safe and healthful working conditions for working men and women" (Occupational Safety and Health Administration 2004). Although the Health Act of 1970 does not specifically require employers to instruct or train employees, Section 5(a)(2) does require each employer to, "comply with occupational safety and health standards promulgated under this Act." A review of 
individual safety and health standards yields more than 100 Acts that do contain training requirements (Occupational Safety and Health Administration 1998).

Recently, the United States Government passed the "Federal Buildings Personnel Training Act of 2010." This act requires personnel performing building operations and maintenance in federal buildings to complete comprehensive training and be able to demonstrate "competency relating to building operations and maintenance, energy management, sustainability, water efficiency, safety (including electrical safety), and building performance measures" (111th Congress of the United States of America 2010). This law was enacted to protect the citizens of the United States, whose tax money is utilized to operate federal buildings, by requiring minimum competencies of the individuals responsible for building performance (U.S. Department of Energy 2012). The safety portion of this bill protects the American taxpayer from worker's compensation claims and lawsuits.

\subsubsection{1. $\quad$ Research Defined Hazard Types}

As stated in Section 1.6 Research Assumptions and Limitations, this research is not intending to address all hazards present in facilities management. Instead, this research focuses on the primary dangers encountered by FM staff that could be mitigated by the developed framework. In order to obtain what dangers are most prevalent for FM staff, a breakdown of fatal and non-fatal data presented by the Bureau of Labor Statistics (2008-2012) is utilized.

The event that caused the fatal and non-fatal accidents is important in order to understand what type of incidents the framework should address. To this end, the framework is not intended to include incidences that have malice intent (i.e. assaults and violent acts) or transportation incidents. Figure 9 presents the event information for fatal events and Figure 10 for non-fatal incidents. In addition to the two figures presenting the number of fatal and non-fatal incidents. 


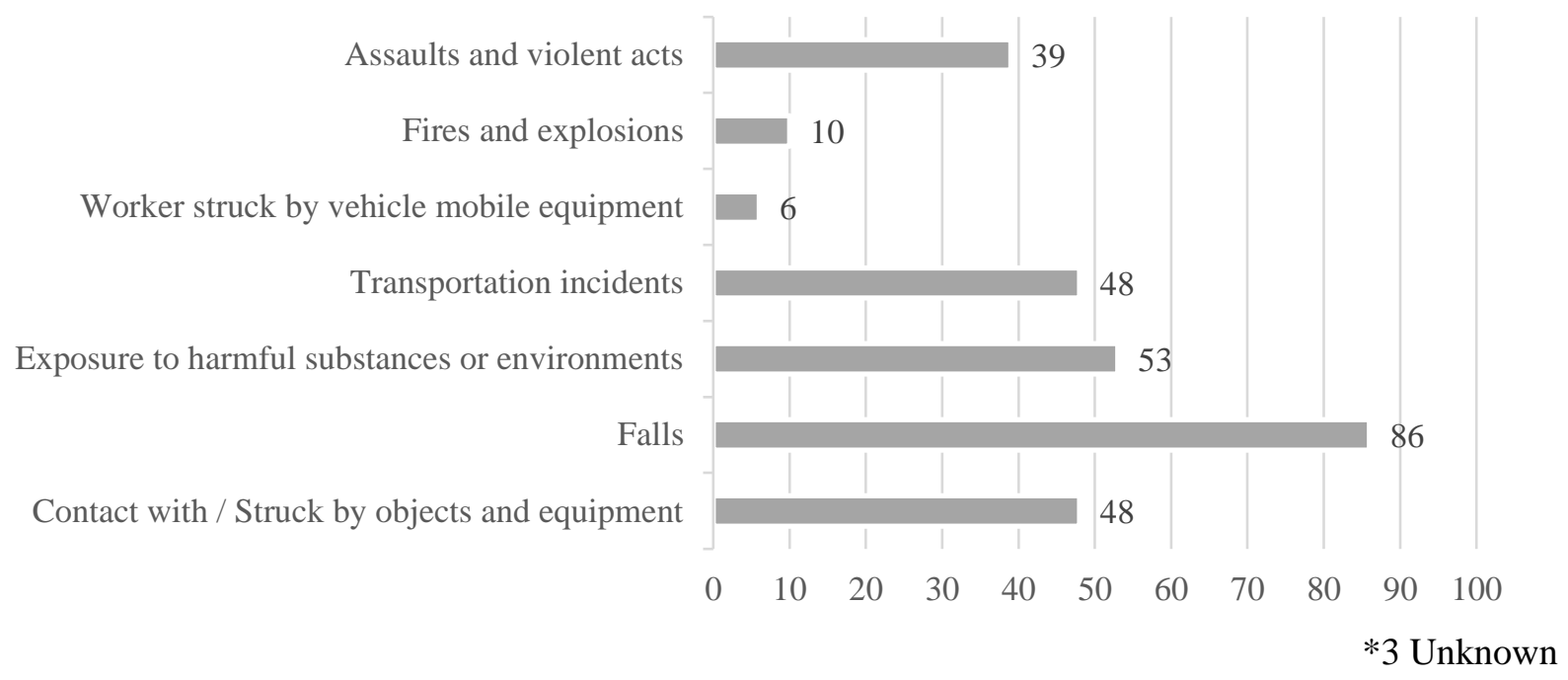

Figure 9: Event Information of FM Fatal Accidents 2008-2012

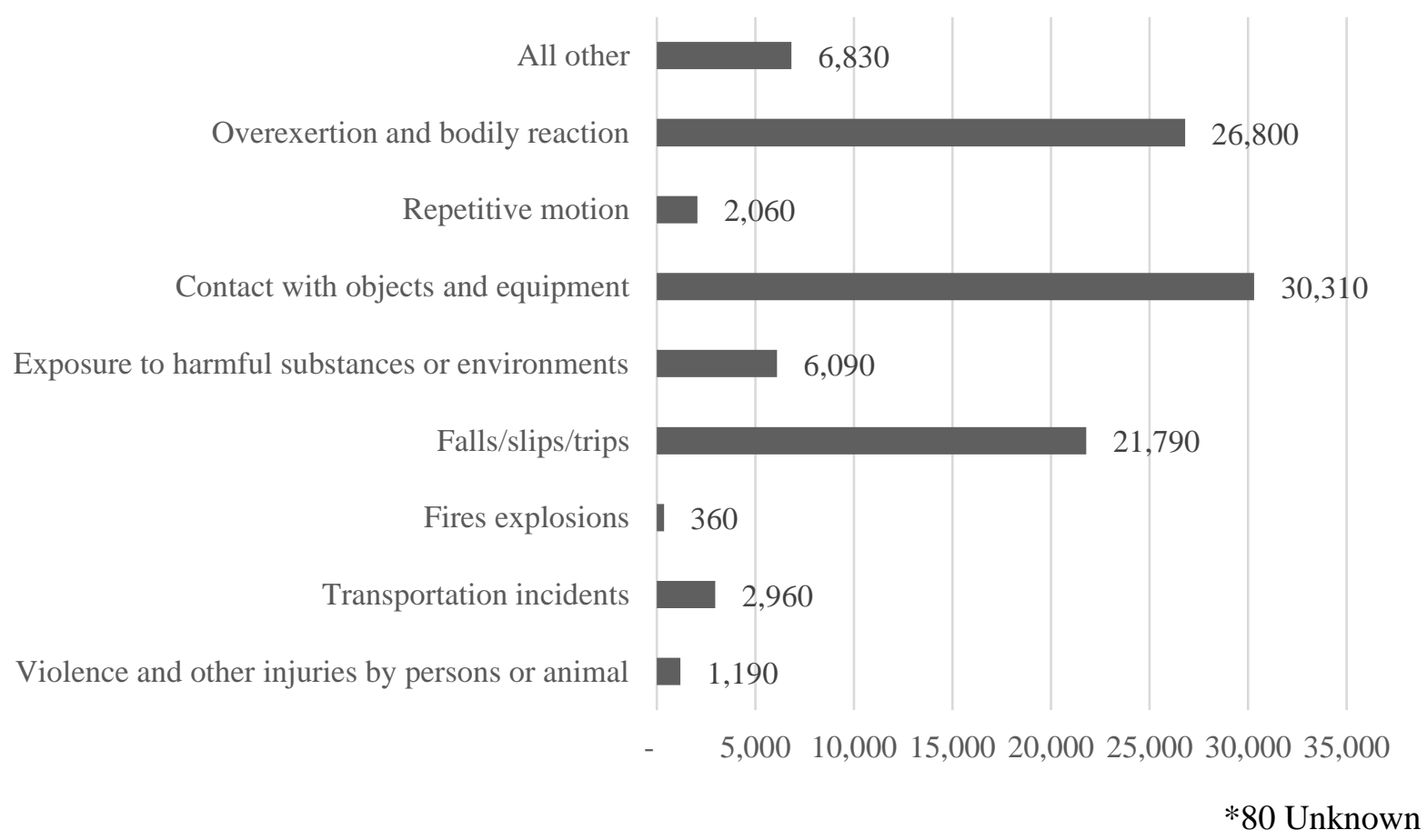

Figure 10: Event Information of FM Non-Fatal Accidents 2008-2012

Analysis of the event data shows that focusing the framework to address falls, exposure to harmful substances and environments (also referred to as hazardous environments and includes electrocution), and contact with / struck by is a relevant direction for the research as these three areas account for roughly $64.5 \%$ of all FM fatalities and $59.1 \%$ of all FM non-fatal incidents. 


\subsubsection{Safety Culture and Human Factors}

Although this research is not intended to address safety culture and human factors, to obtain a comprehensive view of safety within the field of facility management, the perception of safety within an organization must be taken into account. The way facility management staff approaches the safety protocol of work activities will certainly depend on experience, training and available documentation, but will also depend on the worker's values, attitudes, and behavior towards health and safety (International Nuclear Safety Advisory Group 1992). Staff attitudes and behaviors towards safety, also known as safety culture, is often a direct reflection of the organization's culture (Crutchfield and Roughton 2014; Guldenmund 2000; Sorenson 2002). Organizations that take a proactive approach towards the safety culture are often more risk aware, informed, honest, adaptable and resilient (Leighton Contractors 2011).

In addition to the legal and moral obligations to prevent injury and preserve life to the best ability of the organization, an investment in safety culture yields financial benefit through reduced lost time and workers compensation expenses (Vredenburgh 2002). For every $\$ 1$ invested on safety and health programs, a company will typically see a return of $\$ 4-\$ 6$ in lower healthcare costs, increased productivity and higher employee morale (Occupational Safety and Health Administration 2014).

The role that human decision making plays in the implementation of safety protocol cannot be understated. Human error, "a deviation from the performance of a specified or prescribed sequence of actions," accounts for $70-80 \%$ of operational accidents (Leveson 2004). This deviation can be due to any number of reasons, from an increase in system complexity to new hazard types. Similar to the role of safety culture, to minimize the amount of human error within a system, effective approaches will address the goals and the motives behind why a human approaches a solution, as well as how that information is presented.

\subsection{Building Information Modeling}

Building Information Modeling (BIM) is the process of creating a computer-generated model containing precise geometry and relevant data for support during construction, fabrication, procurement and facility lifecycle (Eastman et al. 2008). The use of BIM during the design and construction phase is widely accepted and studies have shown a number of benefits to implementing BIM early in a project (Bryde et al. 2013; Yan and Damian 2008). From reduction in design cycle time to a direct impact on construction cost, the use of BIM is slowly replacing the 2-D hand drafting and CAD applications that have been an industry standard for decades (Epstein 2012; Holness 2006). The term "building information modeling," can be dated back nearly twenty years, while the approaches and methodologies that are identifiable with BIM were presented nearly thirty-five years ago (Eastman et al. 2008). Although BIM has been around for two 
decades, the functionality and features of the software have vastly improved as computer technology has evolved.

Today the marketplace boasts dozens of BIM software applications based on the scope the modeler requires. Table 4 provides a short list of the software available in different categories. This table is not intended to be exhaustive, but to provide an idea of the variety of options available in today's marketplace.

Table 4: Available BIM Software by Category - Adapted from (Broquetas 2010)

\begin{tabular}{|l|l|l|}
\multicolumn{1}{|c|}{ Design } & \multicolumn{1}{c|}{ Sustainability } & \multicolumn{1}{c|}{ Construction } \\
\hline Autodesk Revit & Graphisoft EcoDesigner & Autodesk Navisworks \\
\hline Graphisoft ArchiCAD & Autodesk EcoDesigner & Solibri Model Checker \\
\hline Bentley MicroStation & Bentley Tas Simulator & Vico Office Suite \\
\hline Tekla Structures & Autodesk Green Building Studio & Bentley ConstucSim \\
\hline
\end{tabular}

As designers, constructors, and owners continue to experience collaborative and financial benefits from BIM, as well as the ease of data sharing and the reduction in design time, it is likely that the industry will begin using BIM for design and construction as the industry standard in lieu of the exception. This shift has already begun with the development of a U.S. National BIM Standard, developed by the National Institute of Building Sciences buildingSMART alliance (2014).

\subsubsection{BIM for Facility Management}

This section explores how building information modeling has been utilized in facility management. Reviewing how current facilities are implementing BIM will provide an account of the abilities and limitations within these systems. Additionally, by studying research being executed presents advancements to the transference of data, the implementation of BIM systems, and solutions to specific industry issues.

With all the success that BIM has experienced during the design and construction phase, efforts to transfer information to the facility lifecycle phase is in its infancy. The utilization of BIM for facility management, also known as BIM FM, is a relatively new usage of building information modeling. Prior to the mid1990 's, to fully utilize the design and construction information during the facility management phase, the issue of data transference needed to resolved. With dozens of design and construction software programs 
on the market, developed by a number of different vendors, interoperability between them was non-existent. In 1995, a consortium of twelve companies called the Industry Alliance for Interoperability (IAI) developed an object-based data model that utilized non-proprietary translators that could read the building information across a number of software platforms. The resulting data model was known as the Industry Foundation Classes or IFC (AECbytes 2004).

Today, IFC is published, maintained, and updated by the buildingSMART alliance. As a vendorindependent, open standard format, IFC is supported by roughly 150 software applications worldwide (buildingSMART International Ltd. 2014; Teicholz 2013). The interoperability of the IFC format allows designers, contractors and owners to utilize different software through the many phases of the building lifecycle without losing data due to the proprietary nature of individual software. The IFC data model is highly complex, but maintains a simple user interface. Because most software applications support the IFC data model, extraction of data for transference is typically an export option. The same ease is available for users attempting to import an IFC data model. As long as the software supports the IFC model, the information and model can be brought in through the import or appending function of the software.

In December 2005, the National Building Information Model Standard (NBIMS) Development Team introduced a component to the standard known as the Construction Operations Building Information Exchange or COBie (East 2007). COBie was released to improve how information is captured during the design and construction phases, and then turned over to the owner for operations and maintenance. COBie utilizes the open data format provided by IFC to attempt to bridge the gap between design, construction, and O\&M by mapping commonality within the FM process. By approaching FM activities with an open source, interoperable set of standardized attributes, users can then customize the data to suit their facility needs.

Utilizing IFC and COBie for interoperability has allowed project teams to transfer design and construction data to owners at the beginning of the facility management phase, the implementation of these processes remains an uncommon occurrence. As Lucas (2012) described, "the AEC (Architecture, Engineering, Construction) industry information exchange through the facility lifecycle is fragmented and the facility management phase of the lifecycle remains the most disconnected from the rest." A study conducted by the National Institute of Standards and Technology (NIST) states that, "An inordinate amount of time is spent locating and verifying specific facility and project information from previous activities" (Gallaher et al. 2004). Many of the issues related to the implementation of BIM for FM is due to the factors addressed in Section 5.2.1 Data Handover Challenges. 
In today's market, owners, researchers, and software developers have all realized the issues related to data transference from the end of construction to the O\&M lifecycle phase. Owners have attempted to mitigate the issues with data transference by developing BIM-FM requirements and writing detailed contracts, BIM oriented specifications, and issuing BIM Management Plans that provide project specific methods in order to deliver facility data in a format that the owner is able to utilize. Researchers such as Lucas (2012), Kiviniemi and Codinhoto (2014), Lin and Su (Lin and Su 2013), and others have attempted to synthesize and bridge the gap in data loss between the end of construction and the beginning of the FM phase in complex buildings. By utilizing data exchange frameworks, analysis, and modeling, researchers are pursuing a seamless interaction between construction and post-construction phases. Software developers such as Bentley Systems are developing intelligent models (i-models) to intake, organize, and present equipment and facility data from a number of varying software sources into a single model (Cleveland 2014). Middleware solutions, such as EcoDomus, act as a bridge between a BIM model or database and an application. These systems have shown promise for sizeable organizations but are relatively expensive (Parsanezhad and Dimyadi 2014). Cheaper alternatives, such as Navistools, Datatools, and iConstruct, are application developments that target a specific task, but are not comprehensive enough to service all data transference needs.

Although the systems and research being utilized are young and still problematic, studies of organizations that have successfully integrated BIM FM to some extent, often sizeable government organizations, have shown promising results for utilizing BIM throughout the facility lifecycle. One such study shows a Return on Investment (ROI) of about 64\%, with a payback period of 1.56 years (Teicholz 2013). These savings are realized through the intelligent use of the data collected through the design and construction phase and the integration of BIM FM to make better and faster maintenance decisions based on the data.

As owners, researchers, designers, and developers continue to make strides in the use of BIM throughout the building lifecycle, emerging technologies could help support the complex and data-driven information required for FM (Teicholz 2013). Cloud computing, mobile computing, RFID/QR technologies, augmented reality, and sensor data could all be incorporated into BIM models to provide real-time information. Additionally, the continued research into semantic interoperability and the use of semantic tools (extended algorithms, weighing and ranking systems, etc.) and ontologies will provide greater knowledge management for construction personnel (See Section 2.3.2. BIM/Product Model and Ontology for examples.). A number of resources into current and future applications of O\&M information and technologies are presented by Sapp (2015) in the Whole Building Design Guide. 


\subsubsection{BIM/Product Model}

A product model uses an object-oriented data structure to formally classify information to support the exchange of data through a mechanism (Eastman 1999). The mechanism utilized within a product model is an ontology, a set of translations for how information behaves within a system (Gruber 1993; Lucas 2012). Ontologies are often developed to identify domain specific vocabulary, structure domain knowledge, and exchange information (Chandrasekaran et al. 1999). By executing an ontology within a product model, a conceptual schema or framework of data can be properly structured and stored.

The use of ontologies and product models within construction have often been used to synthesize the causeconsequence sequences that are prevalent within the construction industry (Shansolketabi 2013). Lucas (2012) utilized a product model and ontology to evaluate the data transference of facility management information within a healthcare environment. Implementation of the product model and ontology allowed for the development of process models that evaluated the systems failures in HVAC equipment. TurkaslanBulbul (2006) developed ontologies and a product model which provided computational support for a standardization of building commissioning procedures. The resultant product model standardized commissioning of air handling units and provided a data exchange framework for building commissioning information. Tsai et al. (2009) presented an ontology-based framework that syndicates building intelligence. The proposed framework provides a system that enriches BIM models with knowledge functions, enabling the system to automatically generate responses to facility issues. Park et al. (Park et al. 2012), developed a construction knowledge retrieval systems using semantic tools to enable construction specific knowledge management. Others, such as Venugopal et al. (2012) and Yang and Zhang (Yang and Zhang 2006), have utilized semantic interoperability and ontologies for model exchanges and the advancement of IFC.

In a few cases, researchers have evaluated safety using ontologies. Zhang et al. (2014) recently presented an ontology-based sematic modeling system to capture construction safety knowledge. The ontology utilizes construction based safety information, such as the Occupational Safety and Health Administration (OSHA) regulation 1926 and the Occupational Injury and Illness Classification Manual, in an effort to enable more effective inquiry into construction site safety knowledge. Shansolketabi (2013) evaluated safety within a facility management application by utilizing "chain of events" analysis to evaluate mechanical failures due to improper maintenance. Within the evaluation, an ontology was developed using cause-consequence chains to enable automatic generation of event sequences for a selected domain. The resultant cause-consequence model provided potential failures of a boiler system if proper maintenance was not executed. 


\subsubsection{The Use of BIM for Safety}

The role of BIM in safety began in 1990 when Hinze and Wiegand (1992) surveyed 35 major U.S. design firms to evaluate their role in construction workers safety, subsequently laying the groundwork for the implementation of safety within BIM. During this time period, CAD was primarily used by designers during the design phase, therefore surveying major design firms in the United States was a natural starting point. The results showed that only a third of the respondents made any design decisions based on contractor's safety.

In 1997, in response to the studies performed by Hinze and Wiegand (1992), Gambatese et al. (1997) developed a computer program titled, "Design for Construction Safety Toolbox." The tool was intended to "assist designers in recognizing project-specific hazards and implementing the design suggestions into a project's design." This program was the first application of "Prevention through Design" (Prevention through Design: Design for Construction Safety 2014). Prevention through Design (PtD) is a concept of, "addressing occupational safety and health needs in the design process to prevent or minimize the workrelated hazards and risks associated with the construction, manufacture, use, maintenance, and disposal of facilities, materials, and equipment" (The National Institute for Occupational Safety and Health (NIOSH) 2013). PtD in Europe and Australia, place the legal burden of safety on all parties involved in the project, not just the contractor as OSHA requires in the United States (Behm 2005; Gambatese et al. 2008; Hecker et al. 2005; Her Majesty Stationary Office 1994).

Gambatese et al. (2005) have shown that PtD can reduce the percentage of incidence that occur on a construction project; however, the majority of $\mathrm{PtD}$ tools are primarily text based stand-alone checklists that often do not incorporate BIM (Ku and Mills 2010). A good example of this is the CHAIR system (Construction Hazard Assessment Implication Review) developed by Workcover (2001). The CHAIR system relates design decisions directly to the facility management phase by using a series of "guidewords" to prompt the project team in identifying safety hazards. In particular, CHAIR 3 identifies guidewords for the maintenance and repair phase. This system was developed to address the UK's requirement for the project team to address construction safety as noted earlier.

Although PtD has been around for almost 20 years, the utilization of BIM applications for safety is a relatively new concept and the research in this field is in its infancy. Ku and Mills (2010) state that using BIM to better address safety considerations via hazard recognition and design optimization could "create a built environment that successfully integrates safer construction processes." Table 5 identifies the 
applicable research in the "BIM for Safety" field, including the examples provided above. The table is being utilized to organize the research into the following categories:

- $\quad$ Phase - The portion of the building lifecycle the research is addressing.

- Area of Research - The correlation between the three hazard categories that this research addresses (falls, contact with/struck by, and hazardous environments) and the research being presented in the table.

○ Other - Denotes a non-specific categorization.

- All - Identifies research that addresses all three categories.

- BIM Technology - The modeling tools or techniques used in order to achieve the research objective.

○ BIM Design - Requires additional design to implement safety feature (i.e. scaffolding, fencing, etc.)

○ BIM 4D - Utilizes 4D technologies (3D model \& Schedule)

○ Rules Algorithm - Utilizes a rule based system to output safety information

- Virtual Reality - Uses VR to visualize a work environment or process

- Design for Safety - Technique uses to forecast safety hazards using a BIM model

BIM for Safety in this research only refers to safety to humans and does not address safety of materials (e.g. structural integrity) or life-safety systems (fire safety).

Table 5: BIM for Safety Research

\begin{tabular}{|c|c|c|c|c|c|}
\hline Phase & $\begin{array}{l}\text { Area of } \\
\text { Research }\end{array}$ & $\begin{array}{c}\text { BIM } \\
\text { Technology }\end{array}$ & Summary & Title & Author \\
\hline Design & All & $\begin{array}{l}\text { Design for } \\
\text { Safety }\end{array}$ & $\begin{array}{l}\text { Survey identifying the } \\
\text { designers role in } \\
\text { construction safety }\end{array}$ & $\begin{array}{c}\text { Role of } \\
\text { Designers in } \\
\text { Construction } \\
\text { Worker Safety }\end{array}$ & $\begin{array}{l}\text { Hinze and } \\
\text { Wiegand } \\
(1992)\end{array}$ \\
\hline Design & All & $\begin{array}{l}\text { Design for } \\
\text { Safety }\end{array}$ & $\begin{array}{l}400+\text { design suggestions } \\
\text { that alert a designer } \\
\text { when a project-specific } \\
\text { safety hazard is } \\
\text { identified }\end{array}$ & $\begin{array}{l}\text { Tool To Design } \\
\text { For Construction } \\
\text { Worker Safety }\end{array}$ & $\begin{array}{l}\text { Gambatese } \\
\text { et al. (1997) }\end{array}$ \\
\hline
\end{tabular}




\begin{tabular}{|c|c|c|c|c|c|}
\hline Phase & $\begin{array}{l}\text { Area of } \\
\text { Research }\end{array}$ & $\begin{array}{c}\text { BIM } \\
\text { Technology }\end{array}$ & Summary & Title & Author \\
\hline Construction & All & $\begin{array}{l}\text { Virtual } \\
\text { Reality }\end{array}$ & $\begin{array}{l}\text { A database of safety } \\
\text { processes is incorporated } \\
\text { into a "virtually real } \\
\text { project" to allow for a } \\
\text { walkthrough to identify } \\
\text { safety hazards and select } \\
\text { accident prevention }\end{array}$ & $\begin{array}{l}\text { Integration of } \\
\text { virtually real } \\
\text { construction } \\
\text { model and } \\
\text { design-for-safety } \\
\text { process database }\end{array}$ & $\begin{array}{l}\text { Hadikusumo } \\
\text { and } \\
\text { Rowlinson } \\
\text { (2002) }\end{array}$ \\
\hline Design & All & $\begin{array}{l}\text { Design for } \\
\text { Safety }\end{array}$ & $\begin{array}{l}\text { A theoretical basis } \\
\text { developed to provide a } \\
\text { tool that architects, } \\
\text { engineers, construction } \\
\text { managers (CMs) and } \\
\text { specialty contractors can } \\
\text { use to estimate the time, } \\
\text { cost, and worker safety } \\
\text { impacts of specific } \\
\text { design and construction } \\
\text { process alternatives for } \\
\text { their projects }\end{array}$ & $\begin{array}{c}\text { The Link } \\
\text { Between Design } \\
\text { and Process: } \\
\text { Dynamic Process } \\
\text { Simulation } \\
\text { Models of } \\
\text { Construction } \\
\text { Activities }\end{array}$ & $\begin{array}{c}\text { Slaughter } \\
(2003)\end{array}$ \\
\hline $\begin{array}{c}\text { Not } \\
\text { Applicable }\end{array}$ & Other & $\begin{array}{l}\text { Virtual } \\
\text { Reality }\end{array}$ & $\begin{array}{l}\text { Using virtual reality for } \\
\text { hazard identification } \\
\text { training in mining } \\
\text { operations }\end{array}$ & $\begin{array}{l}\text { Implementation } \\
\text { and evaluation of } \\
\text { a VR task-based } \\
\text { training tool for } \\
\text { conveyor belt } \\
\text { safety training }\end{array}$ & $\begin{array}{l}\text { Lucas and } \\
\text { Thabet } \\
\text { (2008) }\end{array}$ \\
\hline Construction & Fall Hazards & BIM 4D & $\begin{array}{l}\text { Uses } 4 \mathrm{D} \text { for site } \\
\text { organization to promote } \\
\text { safety against falls }\end{array}$ & $\begin{array}{l}\text { BIM-based site } \\
\text { layout and safety } \\
\text { planning }\end{array}$ & $\begin{array}{l}\text { Sulankivi et } \\
\text { al. (2009) }\end{array}$ \\
\hline Construction & All & $\begin{array}{l}\text { BIM 4D / } \\
\text { Rules } \\
\text { Algorithm }\end{array}$ & $\begin{array}{l}\text { A conceptual model } \\
\text { that enables forecasting } \\
\text { of safety risks in projects } \\
\text { for different trades. Uses } \\
\text { a knowledge base of } \\
\text { construction activities } \\
\text { and probabilities of loss- } \\
\text { of-control events, } \\
\text { coupled with a project's } \\
\text { construction plan and a } \\
\text { digital building model, } \\
\text { to forecast risk levels for } \\
\text { work teams }\end{array}$ & $\begin{array}{l}\text { 'CHASTE': } \\
\text { construction } \\
\text { hazard } \\
\text { assessment with } \\
\text { spatial and } \\
\text { temporal } \\
\text { exposure }\end{array}$ & $\begin{array}{l}\text { Rozenfeld et } \\
\text { al. (2009) }\end{array}$ \\
\hline
\end{tabular}




\begin{tabular}{|c|c|c|c|c|c|}
\hline Phase & $\begin{array}{l}\text { Area of } \\
\text { Research }\end{array}$ & $\begin{array}{c}\text { BIM } \\
\text { Technology }\end{array}$ & Summary & Title & Author \\
\hline Construction & Fall Hazards & $\begin{array}{l}\text { BIM 4D / } \\
\text { Rules } \\
\text { Algorithm }\end{array}$ & $\begin{array}{l}\text { A rule based system that } \\
\text { analyzes design } \\
\text { information to } \\
\text { automatically detect } \\
\text { working-at-height } \\
\text { hazards }\end{array}$ & $\begin{array}{l}\text { An integrated } \\
\text { safety } \\
\text { management with } \\
\text { construction } \\
\text { management } \\
\text { using 4D CAD } \\
\text { mode }\end{array}$ & $\begin{array}{l}\text { Benjaoran } \\
\text { and Bhokha } \\
\quad(2010)\end{array}$ \\
\hline $\begin{array}{c}\text { Not } \\
\text { Applicable }\end{array}$ & Other & BIM Design & $\begin{array}{l}\text { Uses computer image } \\
\text { generation for job } \\
\text { simulation (CIGJS) to } \\
\text { review potential safety } \\
\text { hazards in occupational } \\
\text { settings. This is not } \\
\text { specifically geared } \\
\text { towards construction, } \\
\text { but could be utilized as } \\
\text { such }\end{array}$ & $\begin{array}{l}\text { Computer image } \\
\text { generation for job } \\
\text { simulation: An } \\
\text { effective } \\
\text { approach to } \\
\text { occupational Risk } \\
\text { Analysis }\end{array}$ & $\begin{array}{l}\text { Patucco et } \\
\text { al. (2010) }\end{array}$ \\
\hline Construction & $\begin{array}{c}\text { Falls \& } \\
\text { Hazardous } \\
\text { Environments }\end{array}$ & $\begin{array}{l}\text { BIM 4D / } \\
\text { Rules } \\
\text { Algorithm }\end{array}$ & $\begin{array}{l}\text { 1) Uses safety codes to } \\
\text { automatically generate } \\
\text { Dynamic Virtual Fences } \\
\text { (DVF) for collision } \\
\text { prevention \& fall } \\
\text { protection } \\
\text { 2) Uses Real-Time } \\
\text { Location Systems } \\
\text { (RTLS) for worker } \\
\text { tracking to provide } \\
\text { warnings when } \\
\text { approaching hazardous } \\
\text { areas }\end{array}$ & $\begin{array}{c}\text { Automatic } \\
\text { Generation of } \\
\text { Dynamic Virtual } \\
\text { Fences As Part of } \\
\text { BIM-Based } \\
\text { Prevention } \\
\text { Program for } \\
\text { Construction } \\
\text { Safety }\end{array}$ & $\begin{array}{l}\text { Hammad et } \\
\text { al. (2012) }\end{array}$ \\
\hline $\begin{array}{l}\text { Design and } \\
\text { Construction }\end{array}$ & All & $\begin{array}{l}\text { Virtual } \\
\text { Reality / } \\
\text { BIM 4D }\end{array}$ & $\begin{array}{l}\text { Explores relationships } \\
\text { between construction } \\
\text { safety and digital design } \\
\text { practices with the aim of } \\
\text { fostering and directing } \\
\text { further research. It } \\
\text { surveys state-of-the-art } \\
\text { research on databases, } \\
\text { virtual reality, } \\
\text { geographic information } \\
\text { systems, 4D CAD, } \\
\text { building information } \\
\text { modeling and sensing } \\
\text { technologies }\end{array}$ & $\begin{array}{c}\text { Construction } \\
\text { safety and digital } \\
\text { design: A review }\end{array}$ & $\begin{array}{l}\text { Zhou et al. } \\
\text { (2012) }\end{array}$ \\
\hline
\end{tabular}




\begin{tabular}{|c|c|c|c|c|c|}
\hline Phase & $\begin{array}{c}\text { Area of } \\
\text { Research }\end{array}$ & $\begin{array}{c}\text { BIM } \\
\text { Technology }\end{array}$ & Summary & Title & Author \\
\hline Construction & Fall Hazards & $\begin{array}{c}\text { Rules } \\
\text { Algorithm / } \\
\text { BIM 4D }\end{array}$ & $\begin{array}{l}\text { Fall hazard safety issues } \\
\text { unknowingly built into a } \\
\text { construction schedule } \\
\text { can be identified by } \\
\text { utilizing Automated } \\
\text { Safety Checking in a 4D } \\
\text { simulation application }\end{array}$ & $\begin{array}{l}\text { Utilization of } \\
\text { BIM-based } \\
\text { Automated } \\
\text { Safety Checking } \\
\text { in Construction } \\
\text { Planning }\end{array}$ & $\begin{array}{l}\text { Sulankivi et } \\
\text { al. (2013) }\end{array}$ \\
\hline Design & Fall Hazards & $\begin{array}{l}\text { BIM Design } \\
\text { / Rules } \\
\text { Algorithm }\end{array}$ & $\begin{array}{l}\text { Algorithms that } \\
\text { automatically analyze a } \\
\text { building model to detect } \\
\text { safety hazards and } \\
\text { suggest preventive } \\
\text { measures to users are } \\
\text { developed for different } \\
\text { cases involving fall } \\
\text { related hazards }\end{array}$ & $\begin{array}{c}\text { Building } \\
\text { Information } \\
\text { Modeling (BIM) } \\
\text { and Safety: } \\
\text { Automatic Safety } \\
\text { Checking of } \\
\text { Construction } \\
\text { Models and } \\
\text { Schedules }\end{array}$ & $\begin{array}{l}\text { Zhang et al. } \\
\text { (2013) }\end{array}$ \\
\hline Construction & All & $\begin{array}{l}\text { Rules } \\
\text { Algorithm }\end{array}$ & $\begin{array}{l}\text { Utilizes construction } \\
\text { based safety } \\
\text { information, such as the } \\
\text { Occupational Safety and } \\
\text { Health Administration } \\
\text { (OSHA) regulation } 1926 \\
\text { and the Occupational } \\
\text { Injury and Illness } \\
\text { Classification Manual, } \\
\text { in an effort to enable } \\
\text { more effective inquiry } \\
\text { into construction site } \\
\text { safety knowledge } \\
\text { through the use of an } \\
\text { ontology }\end{array}$ & $\begin{array}{l}\text { Ontology-Based } \\
\text { Semantic } \\
\text { Modeling of } \\
\text { Safety } \\
\text { Management } \\
\text { Knowledge }\end{array}$ & $\begin{array}{l}\text { Zhang et al. } \\
\text { (Zhang et al. } \\
\text { 2014) }\end{array}$ \\
\hline Construction & All & $\begin{array}{c}\text { Rules } \\
\text { Algorithm }\end{array}$ & $\begin{array}{l}\text { Identifies focal points of } \\
\text { occupational accidents } \\
\text { as well as risks \& } \\
\text { hazards influencing the } \\
\text { safety of construction } \\
\text { workers. Determines the } \\
\text { job hazards related to } \\
\text { construction process. } \\
\text { After linking to a 3D } \\
\text { building model, the } \\
\text { results are demonstrated } \\
\text { with the commercial } \\
\text { BIM software ceapoint } \\
\text { desiteMD }\end{array}$ & $\begin{array}{l}\text { Model-Based } \\
\text { Construction } \\
\text { Work Analysis } \\
\text { Considering } \\
\text { Process-Related } \\
\text { Hazards }\end{array}$ & $\begin{array}{l}\text { Melzner et } \\
\text { al. (Melzner } \\
\text { et al. 2013) }\end{array}$ \\
\hline
\end{tabular}




\begin{tabular}{|c|c|c|c|c|c|}
\hline Phase & $\begin{array}{c}\text { Area of } \\
\text { Research }\end{array}$ & $\begin{array}{c}\text { BIM } \\
\text { Technology }\end{array}$ & Summary & Title & Author \\
\hline Construction & Fall Hazards & $\begin{array}{l}\text { BIM Design } \\
\text { / BIM 4D }\end{array}$ & $\begin{array}{l}\text { Investigated the } \\
\text { effectiveness of BIM } \\
\text { technologies in } \\
\text { developing, } \\
\text { communicating and } \\
\text { implementing a } \\
\text { construction site safety } \\
\text { plan. Four-dimensional } \\
\text { (4D) phasing } \\
\text { simulations, 3D walk- } \\
\text { throughs and 3D } \\
\text { renderings were utilized } \\
\text { for identifying hazards } \\
\text { and communicating } \\
\text { safety management plan } \\
\text { to the workers }\end{array}$ & $\begin{array}{l}\text { A BIM-based } \\
\text { Approach for } \\
\text { Communicating } \\
\text { and } \\
\text { Implementing a } \\
\text { Construction Site } \\
\text { Safety Plan }\end{array}$ & $\begin{array}{c}\text { Azhar and } \\
\text { Behringer } \\
\text { (Azhar and } \\
\text { Behringer } \\
\text { 2013) }\end{array}$ \\
\hline
\end{tabular}

The following tables present the "Area of Research" and "BIM Technology" usage within Table 5. Table 6 presents the categories identified under "Area of Research" and the ratio to the total percentage of each area, while Table 7 does the same with "BIM Technology."

Table 6: Categorization of Area of Research and Percentage of Each Category

\begin{tabular}{|l|c|}
\hline \multicolumn{1}{|c|}{ Area of Research } & Percent of Literature \\
\hline All (8) & $50 \%$ \\
\hline Falls Only (5) & $31 \%$ \\
\hline Other (2) & $13 \%$ \\
\hline Falls and Hazardous Environment & $6 \%$ \\
\hline
\end{tabular}


Table 7: Categorization of BIM Technology and Percentage of Each Category

\begin{tabular}{|l|c|}
\hline \multicolumn{1}{|c|}{ BIM Technology } & Percent of Literature \\
\hline BIM 4D (7) & $30 \%$ \\
\hline Rules Algorithm (7) & $30 \%$ \\
\hline BIM Design (3) & $13 \%$ \\
\hline Design for Safety (3) & $13 \%$ \\
\hline Virtual Reality (3) & $13 \%$ \\
\hline
\end{tabular}

By mapping the "Area of Research" to the "BIM Technology," tools and techniques can be correlated to issues that this research is addressing. Figure 11 presents this mapping in order to organize the findings so it can be utilized during the development of the proposed framework (see Section 4). Line weights indicate the frequency of correlations.

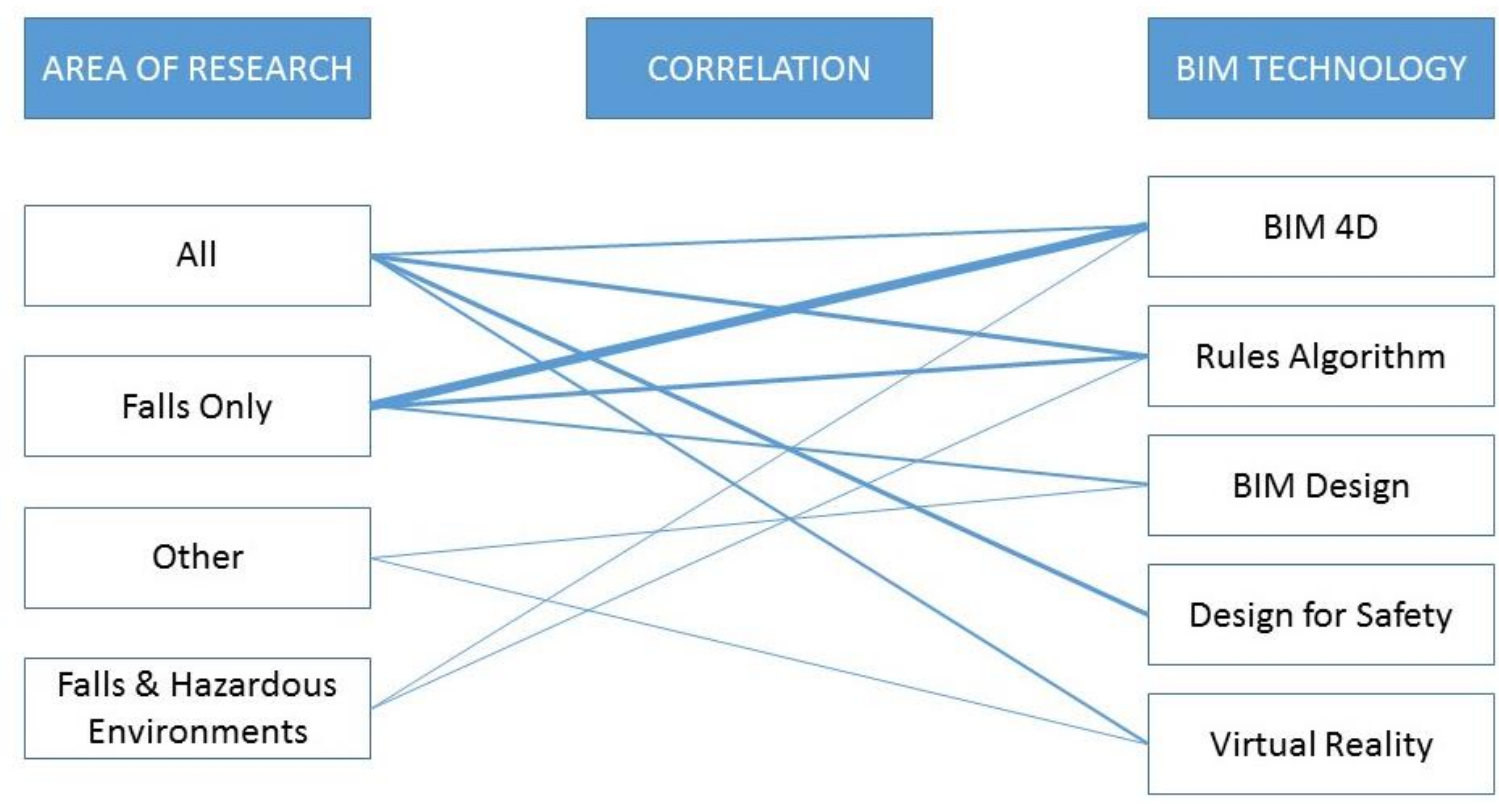

Figure 11: Correlation of "Area of Research" and "BIM Technology"

\subsection{Analysis of Literature Review}

As the FM industry continues to see incidence rates well over the national occupational average and an upward trend in fatalities, a fundamental shift in how safety information is disseminated and presented must take place. As the literature review has shown, a great deal of documentation has been produced in order to create a safe working environment for FM workers; however, this documentation only adds to the problem by creating even more sources of information requiring extensive reference prior to the start of an FM task. 
The incorporation of BIM into facilities management has attempted to improve where the documentation is stored and how it is presented but is rarely utilized due to "handover issues." Additionally, this information is rarely safety oriented, but instead is more asset and O\&M based. Research being done to integrate BIM and safety has shown promise; however, a substantial amount of this research has been geared towards a safe working environment during the construction phase. As a result of the analysis conducted on current trends and technologies, the following summarizes the current challenges faced in each of the three areas of investigation.

Bureau of Labor Statistics data has shown, the upward trend of accidents within the field would indicate that FM workers are not executing tasks utilizing the appropriate safety information. This could be due to any combination of factors, including availability of information, safety culture, time constraints, or expertise. By adding convenience in obtaining information and simplifying the interface with which that data is presented, the likelihood of reference, retrieval, and execution for the three hazard types this research is addressing will improve. This increased convenience will shorten the amount of time and effort an individual must spend in obtaining comprehensive safety information, expediting the reference timeframe and providing more time for the execution of the task. Additionally, simplifying the process should improve the worker's attitude toward referencing the safety information, thus positively shifting the culture.

Software advancements and research done in building information modeling in regards to facilities management has made immense steps within the last decade. The issues with data transference has been considered and continues to be addressed today. Although these systems are not seamless and the industry still experiences issues with data capture and transference, through advancements in IFC, COBie, i-models, middleware, and research, the flow of information at the completion of a construction project into the FM phase is more streamlined than ever before. To date, much of this data management has focused on the flow of O\&M information, construction as-builts, and asset management, with very few cases focusing on the identification and subsequent transference of relevant safety information. By proactively establishing a protocol for safety, based on the equipment and environment present within the facility and structured within the BIM model, the information that is important to FM personnel can be obtained and presented independently in a BIM-based format, without the need to syphon through significant amounts of information.

Based on the information reviewed in the current market literature, analysis of the utilization of BIM for safety during the FM phase, shows none of the available literature reviewed has focused on the FM phase. However, analyses of the literature can help identify tools and techniques that could be expanded to consider 
the FM phase. By identifying what tools/techniques are being utilized and how those tools/techniques correlate to the hazards that this research attempts to mitigate, parallels to the framework that this research is developing can be made and potentially implemented within the system.

As a result of the analysis conducted on current literature, the following Table 8 summarizes the current challenges faced in each of the three areas of interest that the proposed framework attempts to address.

\section{Table 8: Current Challenges This Research Attempts to Address}

\begin{tabular}{|l|l|}
\hline \multirow{2}{*}{ Safety During Facility Management } & $\begin{array}{l}\text { Information is often fragmented creating } \\
\text { inconvenience in obtaining comprehensive } \\
\text { safety related information. }\end{array}$ \\
\hline \multirow{2}{*}{ BIM for Facility Management } & $\begin{array}{l}\text { Handover/Data transference issues are still } \\
\text { prevalent. }\end{array}$ \\
\cline { 2 - 2 } & Handover information is rarely safety based. \\
\hline BIM for Safety & $\begin{array}{l}\text { Research is heavily focused on the design and } \\
\text { construction phase. }\end{array}$ \\
\hline
\end{tabular}




\section{RESEARCH METHODOLOGY}

This research aims to support safety during the facility management phase by identifying safety relevant information applicable to facility management and delivering that data in a singular BIM-based framework. The methods used in order to execute the research goals is based on qualitative analysis through the theoretical lens of a Six Sigma methodological approach. Define-Measure-Analyze-Design-Verify (DMADV), is a popular phased analytical tool used for the development of processes, services, or products under the Six Sigma methodology, Design for Six Sigma (DFSS) (Rumane 2013). The framework focuses on the development of processes, services, or products through end-user interaction. This interaction can be expressed through qualitative processes (i.e. interviews, case studies, etc.) (Narayanasamy 2015), as is the case in this research. Logan et al. (2005) states,

"Design and new product development often involves qualitative goals and depends on advances in research and development yet to come to fruition... The Design for Six Sigma process is ideally suited to deal with both qualitative and quantitative aspects, and has the added advantage of a smooth transition into the product phase use of Six Sigma principles."

The DMADV framework is flexible enough to be utilized exclusively as a qualitative method or as a mixed methods framework, as presented by Mahasneh (2014). Although frequently implemented in the manufacturing and business worlds, the use of Six Sigma methodologies has been shown to be an effective approach in construction research (Banawi 2013; Koziolek and Derlukiewicz 2012; Lee and Su 2013; Mahasneh 2014; Paslawski 2013; Vilasini et al. 2014).

By structuring the research within the DMADV framework, the methods utilized for data collection, organization, and presentation can be anchored in a tested methodology for the deliverables developed within this research. As Six Sigma is primarily a tool for quality in the manufacturing of products, a correlation to the development of a framework can be extrapolated. By using the steps identified under DMADV, the research design and execution is continuously gearing the development of the framework towards the end user and the end user's needs. In this research, the end user is facility management staff and the end user's needs are the retrieval of safety related information efficiently. Combining the theoretical framework with the researcher's experience and existing literature provides a conceptual framework that, as described by Rossman and Rallis (2012), "underscores the interaction between the inductive (reasoning from the particular to more general statements to theory) and deductive (starting with theory and testing its applicability) processes of research." Figure 12 provides a visual representation of how the DMADV 
theoretical framework will be incorporated into this research. Details of the framework and the methods utilized to execute this methodology are provided in Section 3.1.

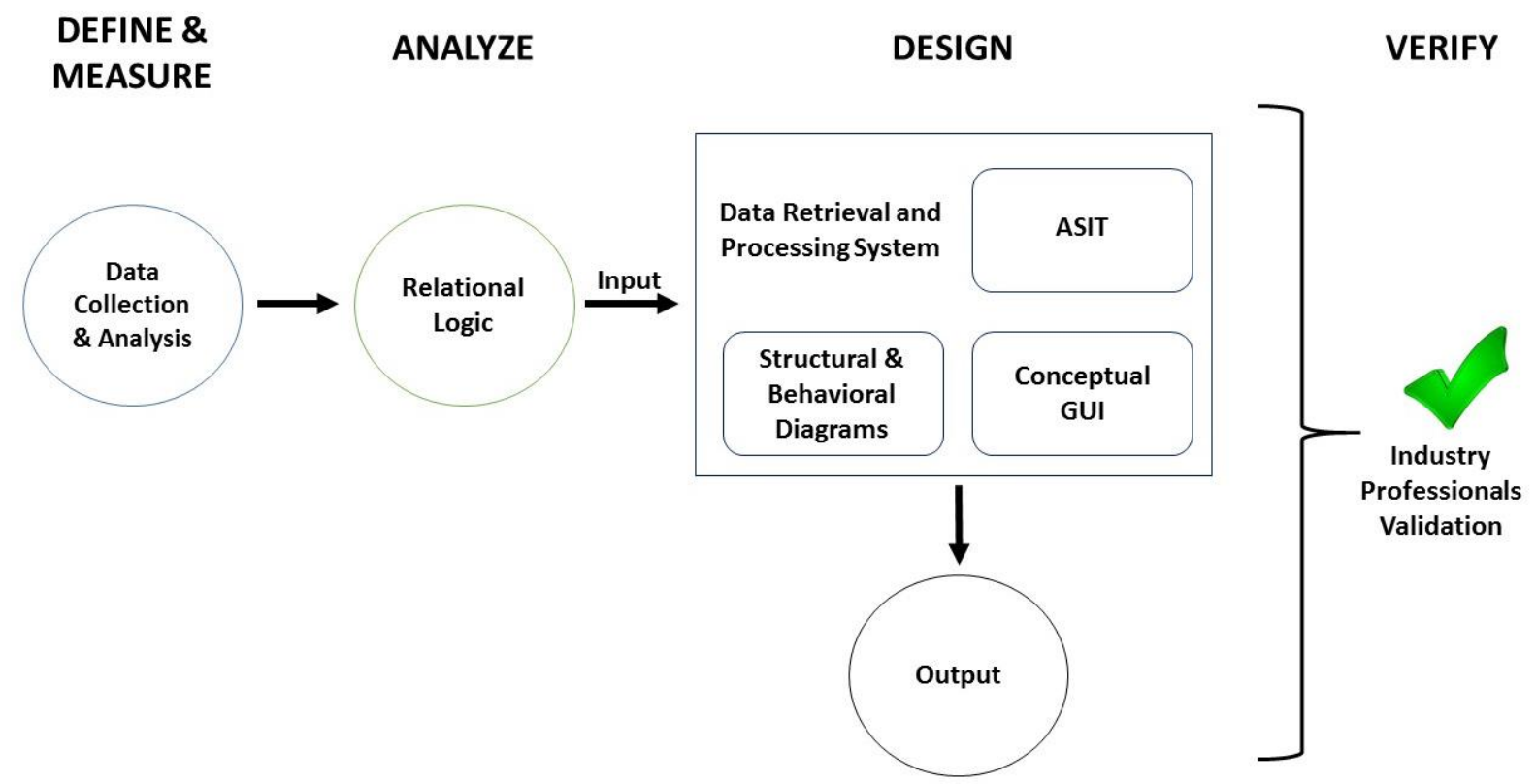

Figure 12: The DMADV Theoretical Framework in this Research

In this chapter, a detailed explanation is presented for the methods this research utilizes within the context of the Six Sigma methodology and the qualitative approach. Section 3.1 provides background on the DMADV framework, presents the methods used to execute research, and presents the research methods within the DMADV theoretical framework, Section 3.2 describes the role of the researcher for this study, and Section 3.3 presents the research trustworthiness and ethical considerations.

\subsection{Research Design}

The following section details the DMADV theoretical framework, identifies the methods being utilized to mitigate the problem statements identified in this research, and describes how these methods are structured within the DMADV theoretical framework. This research utilizes qualitative analysis during data collection in the form of case study analysis, coding of safety related information, and interviews. Data collection is validated by a second round of interviews with industry professionals. The coded data is then organized through mind mapping, standardized through a developed tool, translated into Unified Modeling Language, and placed into a product model and sequence diagram. A conceptual graphical user interface (GUI) utilized in correlation with the diagrams, provides a proof of concept to the system functionality. 


\subsubsection{Theoretical Framework Background}

The Six Sigma methodology was initially developed by Motorola in the mid 1980's to reduce defects in the manufacturing process (Tjahjono and Ball 2010). Initially, Six Sigma was heavily quantitatively based, with a singular goal of minimizing manufacturing defects to 4 Defects per Million (Logan 2005). Since that time, the use of this methodology has expanded into multiple industry sectors and is still being implemented in new sectors today. Additionally, the Six Sigma methodology, has expanded far beyond a single quantitative metric into mixed and qualitative methodologies. Six Sigma is most often utilized when improving the quality and organization of an existing process or product through the use of DefineMeasure-Analyze-Improve-Control (DMAIC). This analytical process provides a systematic approach to the evaluation and continuous improvement to optimize an existing process or product (Koziolek and Derlukiewicz 2012; Sokovic et al. 2010). The DMAIC system has been shown to be an effective tool for existing process/products, but when a new process is to be developed, as is the case in this research, a different approach should be utilized.

Design for Six Sigma (DFSS) has become a worthy predecessor to DMAIC in the application of new process/product development under the Six Sigma methodology (Logan 2005). One of the most frequently reported methodologies for implementing DFSS is the use of the Define-Measure-Analyze-Design-Verify or DMADV (Sokovic et al. 2010). Similar to DMAIC, the DMADV system uses a systematic approach to develop products that have been verified through interaction with the end user. Each phase within the DMADV methodology has a specific task. These phases are defined in the context of this research in Table 9. A full description of the methods utilized in this research and how those methods are structured within the DMADV methodology is demonstrated in the Section 3.1.3.

Table 9: Definition of the DMADV Methodology in the Research Context

\begin{tabular}{|c|c|}
\hline Phase & Definition \\
\hline Define & Identification of the data and information for the stated goals. \\
\hline Measure & $\begin{array}{l}\text { Identification of "benchmark" and verification of data collected during the Define } \\
\text { phase. }\end{array}$ \\
\hline Analyze & $\begin{array}{l}\text { Qualitative Analysis of the information gathered during the Define and Measure } \\
\text { phases. }\end{array}$ \\
\hline Design & $\begin{array}{l}\text { Implementation of the knowledge gained through the Define, Measure, and Analyze } \\
\text { phases. }\end{array}$ \\
\hline Verify & A third party validation of the designed system. \\
\hline
\end{tabular}




\subsubsection{Research Methods for Proposed Framework}

The proposed BIM-based framework and stages are based on a qualitative analysis through the theoretical lens of a Six Sigma methodological approach. Define-Measure-Analyze-Design-Verify (DMADV), is a popular phased analytical tool used for the development of processes, services, or products under the Six Sigma methodology, Design for Six Sigma (DFSS) (Rumane 2013). The BIMbased framework focuses on the development of processes, services, or products through end-user interaction.

The DMADV theoretical framework is used to provide a structure for the methods of data collection and the development of the framework. Figure 13 presents the research methods within the DMADV theoretical framework. The first column in Figure 13 presents the DMADV phase, the second column defines the phase, developed from The Pennsylvania State University (2008), and the third column presents the research methods utilized within this research in the DMADV context. 


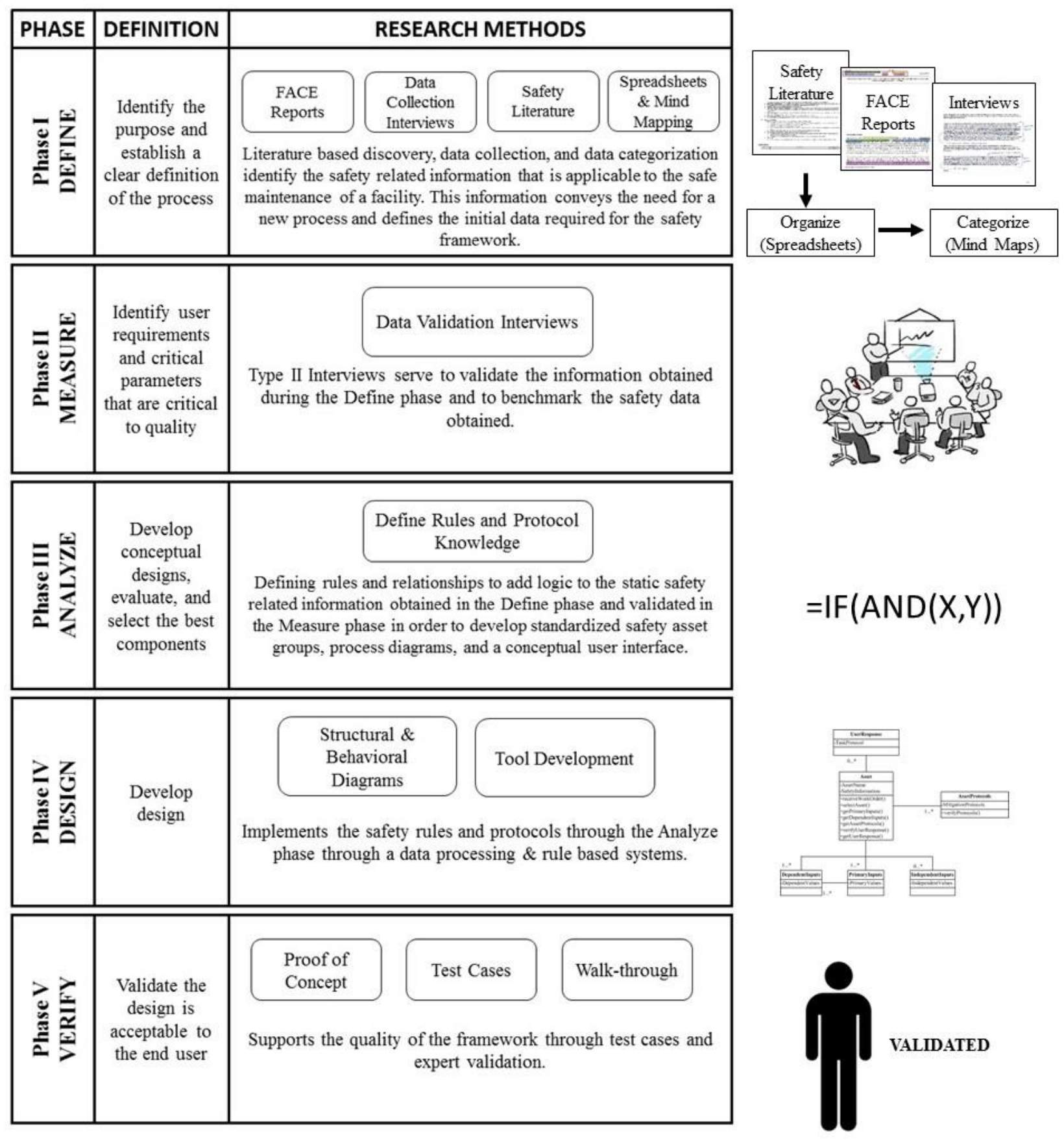

Figure 13: Research Methods within the DMADV Framework 


\section{Phase I: Define}

Using Literature based discovery and data collection, the safety related data that is applicable to the safe maintenance of a facility is identified. In order to obtain safety data, case study analysis of FACE reports, semi-structured open-ended interviews (data collection), and safety related information, such as O\&M Manuals, safety handbooks, OSHA handbooks, etc. are analyzed through qualitative coding. Analysis of this information provides safety data that is utilized to develop safety inputs. The safety data is first organized through Excel Spreadsheets in order to identify commonalities and then categorized using Mind Maps to present a hierarchy of the static information.

\section{Phase II: Measure}

Data Validation Interviews executed during the Measure phase serve to validate the information obtained during the Define phase. In this phase, the interviewee benchmarks the critical parameters and validates that the data obtained in the Define phase is comprehensive, accurate, and commensurate with industry standards.

\section{Phase III: Analyze}

Based on the safety inputs identified in the Define phase and validated through the Measure Phase, relational logic is developed using conditional constructs in order to further structure the safety inputs and begin to define the functionality of the BIM-based framework.

\section{Phase IV: Design}

Develop an approach to standardize asset specific safety properties and deliver the properties from its point of origin to the end user in a singular BIM-based repository. Develop a system to retrieve and process the safety properties in order to be presented to the end user within a conceptual graphical user interface (GUI).

\section{Phase V: Verify}

Supports the quality of the framework that this research develops. Utilizing a Proof of Concept model, test cases, and industry expert walk-throughs to verify the functionality of the developed safety inputs, ASIT, and DRPS. Items from the verify phase will be utilized in future research and further development of the framework.

\subsubsection{Research Methods}

This section presents the research methods being utilized within this research in order to execute the objectives stated in Section 1.5. 


\subsubsection{Data Collection and Organization-Objective No. 1}

Case Study Analysis is an intensive investigation of people, organizations/institutions, events, and occurrences, used to identify phenomena, themes, concepts, or principles from which a theory can be developed or practice improved (Fritz 2008). This research utilizes a modified paradigmatic explanatory multiple case study analysis in the evaluation of Fatality Assessment and Control Evaluation (FACE) reports to illustrate common patterns or themes (Yin 2003). The following bullet points will explain each modifier presented under the type of case study analysis being utilized:

- Modified - The case study analysis in this research analyzes the information that the FACE Reports are presenting in order to pull data points (or codes) from these reports.

- Paradigmatic - A type of case study analysis that uses careful selection of examples to reveal key elements of a phenomenon (Pavlich 2010). This research only uses cases regarding occupational injuries, illnesses, and fatalities to workers in the field of facility management as a result of falls, contact-with/struck-by, or exposure to harmful substances/environments.

- Explanatory - Case study analysis focused on the "how" and "why" a phenomena occurred (Yin 2003).

- Multiple - Research that utilizes more than one case study, is known as a "multiple." This research will utilize a number of FACE Reports to explore differences and similarities within and between cases (Baxter and Jack 2008; Yin 2003).

This research has identified case study analysis as the best method of data extraction from the FACE Reports for a number of reasons. These reasons are presented below:

- Detailed accounts of existing real-life events are available from a reliable source.

- Relevant behaviors cannot be manipulated.

- Direct observation is not viable as events cannot be safely recreated.

- Case study analysis will be combined with safety related documentation and interviews to create "triangulation" in order to develop converging lines of inquiry.

The FACE reports provide an understanding of "how" and "why" fatalities are occurring in the field of facilities management. This information is critical to the execution of Objective No. 1, by identifying FM applicable safety data and the subsequent use of that data to develop safety protocols. In order to achieve this objective, multiple case studies falling under the categories being investigated by this research will be reviewed in order to develop safety codes through qualitative coding. These codes are pieces of information, relevant to the "how" and "why" an accident occurred. Using thematic analysis to evaluate code 
frequencies, co-occurrences, and relationships, the codes can be organized and categorized to support analytical generalizations (Guest et al. 2012; Robson 2011). The FACE Reports are coded by hand or within a .pdf markup software. Codes are placed into an Excel spreadsheet for organization, prior to being further organized utilizing mind mapping.

Each case study reviewed for this research set out to answer four questions. Under what circumstances did the accident occur? (2) Could the accident have been avoided? (3) What measures could have been taken to improve the safety? (4) Could these measures be applied to multiple situations?

Qualitative Coding will also be used as a source of information to develop safety codes from industry standard literature, such as, Operations and Maintenance Manuals, Safety Handbooks, OSHA handbooks, etc. Qualitative coding uses the, "analytical process of organizing raw data into themes that assist in interpreting the data" (Baralt 2012). For this research, qualitative data will be hand coded and placed into an Excel spreadsheet for organization, prior to being further organized utilizing mind mapping. Similar to the case study analysis and interviews, thematic analysis is utilized to find the relevant safety information needed to execute this study. Data collection from industry standard literature requires the use of constructivist theory. The data pulled from these documents is often uncategorized and therefore is at the discretion of the author of the data's importance. This information is later validated through Data Validation Interviews.

Semi-structured, open-ended interviews - Data Collection Interviews with experts in the field of facility management will aid in the compiling and categorization of applicable safety data. Similar to the other data collection methods, Data Collection Interviews are intended to provide codes through qualitative coding of transcripts and thematic analysis. These codes will then be incorporated into the framework, similar to the codes identified through the FACE Reports and safety literature. This research utilizes data saturation for the three hazard areas and therefore the number of participants required is unknown. Interviews are not be fully structured and appear more as a guided conversation rather than structured queries. This method allows for a more fluid/dynamic interview where a consistent line of inquiry is being pursued, however, the format provides an opportunity for follow-up questions and parallel lines of inquiry (Yin 2003). The sampling of interviewees will be purposive, by targeting a particular group of people based on the criteria of knowledge of facility management and safety applications. Participants in this study meet the following inclusion criteria:

- Facility management professional - This could include field staff or supervisory staff

- Over 18 years old 
- Minimum 6 months working at current position - This is to avoid interviews with trainees or individuals not well versed in the field

- Able to speak and read English

The inclusion criteria this research utilizes was strategical situated to obtain qualitative data from FM experts. Gathering safety data from experts provides, in combination with the other data sources, a comprehensive list of safety inputs and protocols.

Semi-structured, open-ended interviews - Data Validation Interviews are used to validate the data compiled through the case study analysis, safety data, and Data Collection Interviews. Data Validation interviews utilize interviewees to validate data saturation and accuracy. Due to the nature of these interviews, the questions asked will be focused more on validation of collected data.

The interviews are audio taped and field notes are taken to allow for qualitative analysis. Interviews last between 60-90 minutes, face-to-face with the lead researcher, at a location identified at interviewee's discretion. If a face-to-face interview is not possible due to travel restrictions, a virtual meeting (i.e. FaceTime or Skype) is an acceptable alternative. Participants are identified by locating facilities that have staff that could meet the inclusion criteria, for example, contacting the administrative office or FM department directly at a water treatment facility, school, university, or factory. Once an FM supervisor is identified at the facility, a phone call will be made to verify the contact information with the supervisor, confirm compliance with inclusion criteria, and obtain an interest level of study participation. If the participant is interested in participating, a recruitment email will be sent outlining the details of the study, as well as other IRB required information. Snowballing, or allowing the selected participant to provide names of other potential participants, is utilized on a needed basis. Upon completion of the interview, participants are debriefed by describing the process of member checking (see Section 3.3.2) and are notified of the right to drop out of the study at any time. Additional information regarding the interviews including the role of the researcher, quality and rigor, informed consent, and confidentiality are discussed in detail in Section 3.2 and Section 3.3.

Mind Mapping is a graphic technique, that expresses radiant thinking, by allowing a user to show relationships among various concepts and ideas on a single page (Buzan and Buzan 1996; Mento et al. 1999). Within a mind map, the subject of attention appears as the central image, with key themes (or words) radiating around the subject as branches. These branches can be represented utilizing a hierarchical system, with items of lesser importance radiating further from the main subject. The mind mapping technique is 
utilized in this research to organize the coded qualitative data received from the case studies, interviews, and safety information. Organizing the data across the three data collection methods provides a singular graphical reference for the coded data. By organizing the information in a graphical hierarchy, the transition into a product model and sequence diagram is simplified. The collection of data through the qualitative analysis and the organization of that data through mind mapping, executes the requirements further discussed in Objective No. 1.

\subsubsection{Data Transfer-Objective No. 2}

Utilizing existing literature and simulated tests on a number of software, a data transference mechanism is identified. Execution of this Objective will aid in the mitigation of "handover issues" that are present within the industry.

\subsubsection{DRPS Development - Objective No. 3}

A Product Model is developed in order to further organize and add logic to the information obtained through the FACE reports, interviews, and safety information. The product model for this research utilizes the Unified Modeling Language (UML) Classification within a Class Diagram for the identified attributes as a static representation of a knowledge base.

Sequence Diagrams, a type of "Behavioral Diagram," presents how objects interact in a particular scenario over a period of time (Pilone and Pitman 2005; Visual Paradigm 2016). For this research, a sequence diagram is developed to present how the product model classes interact and the system retrieves the relevant safety information.

\subsubsection{Conceptual GUI Development-Objective No. 3}

Using Java Eclipse, a conceptual representation of the graphical user interface (GUI) for the system is developed. The conceptual GUI is utilized to visually present the interaction between the FM worker and the DRPS.

\subsubsection{Validation Methods-Objective No. 4}

Utilizing the Conceptual GUI in coordination with the UML Class and Sequence Diagrams, a Proof of Concept is presented through Test Cases.

Cognitive Walk-Through is utilized to present the proof of concept model to an industry expert in order to validate the developed system. 


\subsubsection{Limitations to Stated Methods}

The following section address the limitations of this study within the context of the above stated methods.

Method Limitation No. 1: Interviews with industry professionals for data collection and validation will take place within driving distance of Blacksburg, VA and therefore may not include considerations applicable to other regions of the United States or internationally.

Method Limitation No. 2: Sample selection is purposive, with specific inclusion criteria, and is not intended to accommodate random sampling. Therefore, it is likely that certain sections of FM staff will not be utilized for data collection or validation.

\subsection{The Role of the Researcher}

The role of the researcher in this research is to study phenomena which has already taken place and evaluate qualitative data from these phenomena in order to provide a solution for the stated problems. To execute this task, the researcher maintains a role of observer and interviewer. The researcher and participants do not attempt recreate or engage in any of the events being evaluated, as this would create a significant safety hazard and would be irresponsible. As an interviewer, discussions take place regarding facility management (FM) protocol and safety with industry experts, recruited based on their knowledge of FM. There is no previous personal or work-related relationship with the individuals and because of this, power relationships are not an issue. In order to mitigate any perceived researcher bias, questions are guided only toward facts and opinions of the interviewee. Interjections by the interviewer only come in the form of follow-up questions/probes and clarifications. A conscious understanding by the researcher of how questions are asked, the tone in which they are stated, and the body language presented during the interview attempt to mitigate any response bias.

The lead researcher in this study has spent 6 years of his professional career working with a number of the individuals and documents that this research utilizes. This include construction documentation, working with Operations staff, project closeout and turnover, and building information modeling. This time in the field has allowed the author to draw on previous experience in order to execute the research being presented in this study. 


\subsection{Research Considerations}

In any research study, considerations for quality and rigor must be made and enacted in order for findings to be valid. These considerations are even more important when the data collection methods for the study are qualitatively based. In addition, the use of human interaction through interviews carries ethical considerations in order to eliminate the potential for harm to the study participants. This section will present the methods that are used in order to maintain a trustworthy and ethical study.

\subsubsection{Trustworthiness Using Qualitative Methods}

Classical science has evolved around a core principal that studies must be refutable and replicable. Qualitative research, by nature, cannot be completely replicable as the data being collected relies on the reflexivity (awareness of self and others) of the researcher and the information being provided by the study participants at that moment in their experience. Anfara et al. (2002) describes this paradigm and offers a solution that has over time, emerged as a staple in qualitative research,

"We operate from the basic premise that how researchers account for and disclose their approach to all aspects of the research process are key to evaluating their work substantively and methodologically. Central to this premise are the core elements of classical science - refutability and replicability. Because one of the "difficulties" with qualitative research is the recognition that it is not, in the "classical science" sense, replicable, we recommend analytic openness on the grounds of refutability and freedom from bias."

To obtain the trustworthiness in qualitative research, Guba and Lincoln (1982) developed now widely accepted criteria as counterparts to the conventional (classical science) terms for assessing research quality and rigor. Table 10 presents the conventional terms utilized in quantitative research and the qualitative counterpart developed by Guba and Lincoln. Additionally, this table defines what each term means in the qualitative sense. 
Table 10: Comparison of Terms for Quality and Rigor (Adapted from Guba and Lincoln (1982)

\begin{tabular}{|l|l|l|}
\hline \multicolumn{1}{|c|}{ Quantitative Term } & \multicolumn{1}{|c|}{ Qualitative Term } & \multicolumn{1}{c|}{ Qualitative Term Definition } \\
\hline Internal Validity & Credibility & $\begin{array}{l}\text { The relationship between the data of an inquiry and } \\
\text { the phenomena those data represent. The data being } \\
\text { produced through qualitative inquiry is providing } \\
\text { believable results. }\end{array}$ \\
\hline External Validity & Transferability & $\begin{array}{l}\text { Provide "thick descriptions" through a vicarious } \\
\text { experience and a transferability of hypotheses to a } \\
\text { second context for the reader. }\end{array}$ \\
\hline Reliability & Dependability & Stability in the data through analytical openness. \\
\hline Objectivity & Confirmability & $\begin{array}{l}\text { Verification of the data through methods of } \\
\text { validation. }\end{array}$ \\
\hline
\end{tabular}

\subsubsection{Trustworthiness in this Research}

This research utilizes standards set forth by Guba and Lincoln during the data collection phase, as well as uses validation methods to maintain a commensurate level of quality and rigor to the overall framework development. During the data collection phase, the terminology Guba and Lincoln presented (credibility, transferability, dependability, and confirmability), can be utilized in order to insure trustworthiness when utilizing qualitative methods. Table 11 presents the means that this research utilizes in order to maintain trustworthiness during data collection and analysis.

Table 11: Means of Trustworthiness in this Research
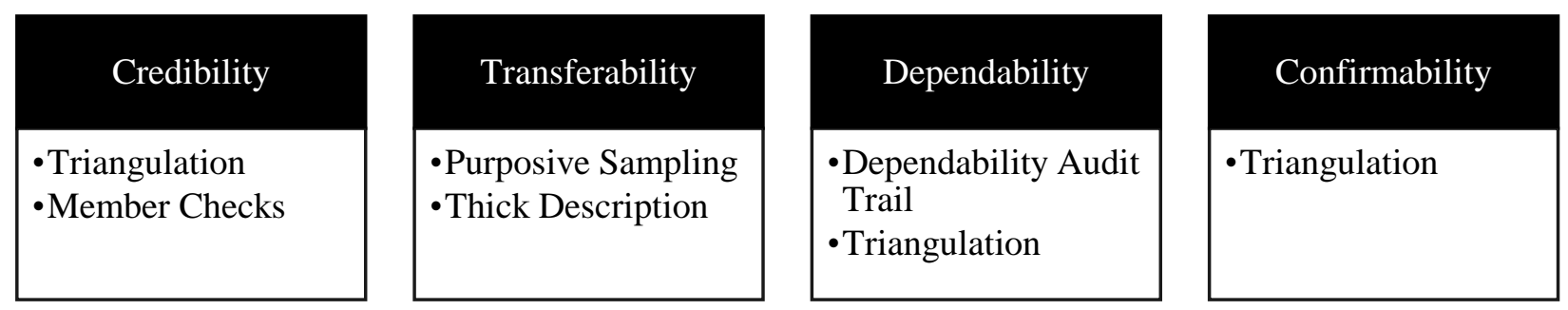

- Triangulation cross-checks data and interpretations by verifying themes across from a variety of data sources. For this research, triangulation will occur by verifying themes across the interviews, case studies, and safety information. For example, FACE Reports are a retelling of how an accident occurred. This type of data is susceptible to human error and omission. Triangulation is utilized in order to mitigate an outlying occurrence in the data by comparing the data point to other sources.

- Member Checks verify the data and interpretations by checking with the individuals who solicited the information. In this study, member checks are used to verify the information gathered during 
the interview phase. At the conclusion of the interview, the data received from the solicitor will be synthesized and presented to the solicitor. Upon review by the solicitor, a written confirmation, in the form of an email, verifies that the data and interpretations were understood and conveyed in a manner in which the solicitor intended. If data or interpretations were misrepresented, the information will be adjusted and noted in the record. Interviewees have two weeks (14 days) from the time the member check is sent out to verify the information. If no response is received by the interviewee within the two-week period, verification of the data and interpretations are implied.

- Purposive Sampling is utilized in this research. See Section 2.3.1 Research Methods for additional information on purposive sampling, including the inclusion criteria for this study.

- Thick Description is a technique used to provide enough information about a context to impart a vicarious experience and a transferability of a hypothesis to a second context for the reader. Thick descriptions are utilized in this research to depict the tone, surroundings, and feel of the interviews.

- Dependability Audit Trail is an accounting of the methodological steps and decisions made regarding the research. When a decision is made regarding the research, that decision is dated and noted in a running word document. The audit trail begins at the acceptance of the research proposal and is maintained throughout the data collection and data analysis phase of the research.

In addition to the means identified for the trustworthiness of the qualitative data collection of this research, a number of additional verifications, as shown in Section 3.1.2 Research Methods, are being utilized to demonstrate quality to the framework that this research develops. Continuous industry expert validation, proof of concepts, and a cognitive walk-through are steps beyond the data collection and analysis in order to validate the developed framework. All of these means are intended to produce a trustworthy study that maintains analytical openness and utilizes feedback from industry professional to validate the framework.

\subsubsection{Ethical Considerations}

This research follows the protocols and standards set forth by the Virginia Tech Internal Review Board (IRB) to verify compliance with the established standards of human research. Following the guidelines set forth by the IRB and following the accepted protocols for human research, ensures this research will not violate any ethical considerations including, informed consent, unwanted dissemination of personal or company information, recruitment, data collection, and data storage. Additionally, the use of methods such as member checks following an interview, eliminates unintended misrepresentation of the interviewee. 


\section{DATA COLLECTION, CATEGORIZATION, AND VALIDATION}

As stated in Chapter 3, data collected in this research utilizes triangulation through Data Collection Interviews, FACE report coding, and safety information coding. This chapter details out how the coded information was obtained and the results from data collection. This information is utilized to develop safety inputs and protocols that are installed within the developed framework. With reference to Figure 14, six steps are defined:

1. The data collection methodology utilized in this research involves qualitative coding of FACE Reports, Data Collection interview transcripts, and safety literature. Using three forms of qualitative data sources allows for triangulation, adding the necessary rigor and validation to the identified safety inputs and protocols.

2. The relevant extracted information from each data source is organized into spreadsheets. The Xaxis in each spreadsheet is specific to the data being collected from each source. However, the Xaxis evolves as more data is collected (see step 6).

3. Organized data is coded and attributes are extracted.

4. Attributes can be segregated into safety inputs and protocols through knowledge development. These inputs and protocols are utilized within the framework for the three hazard types that this research is addressing; falls, exposure to harmful substances and environments, contact with/struck by.

5. Patterns and commonalities emerge among the three data sources.

6. As more sources were coded, patterns began to emerge and commonalities within the three sources became apparent. As patterns between the data sources began to link, an iterative update of the spreadsheet headings was executed in order to further process the data.

The following sections provide details to the data collection sources and examples of how safety inputs and protocols are extracted from the text for each of the three sources of relevant safety information. 


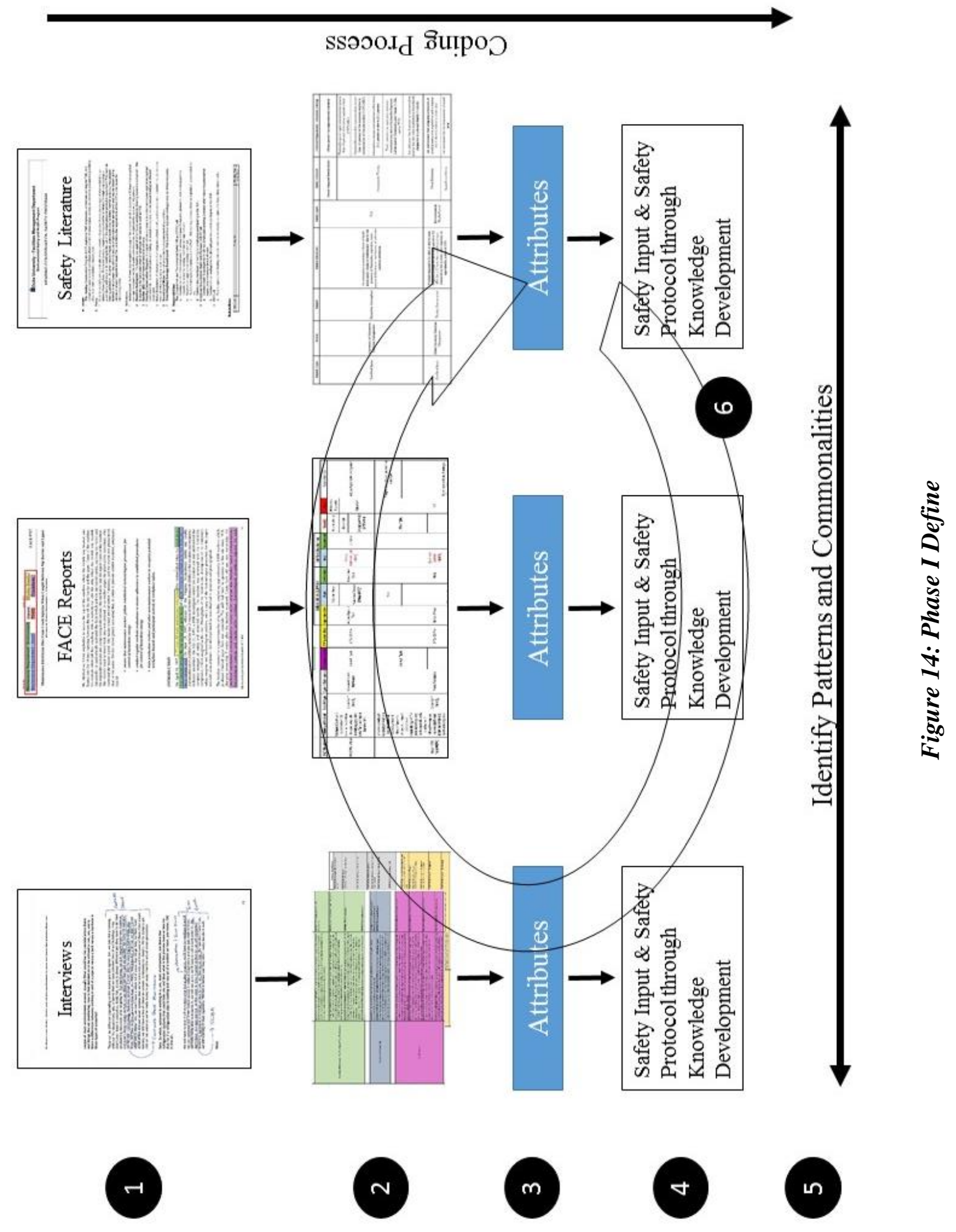




\subsection{FACE Reports}

Fatality Assessment and Control Evaluations or FACE Reports (National Institute for Occupational Safety and Health 2014), provide detailed reviews of fatal accidents that take place in a work environment. Through the FACE program, the National Institute of Safety and Health (NIOSH) conducts investigations into employer-reported fatal incidence. These detailed reports provide demographic, work environment, accident, and prevention information for each incident. In order to utilize FACE reports for this research, a comprehensive review of the 613 records available for review on the Center for Disease Control and Prevention - NIOSH website was completed, identifying facility management specific incidences for detailed evaluation (National Institute for Occupational Safety and Health 2014). Of the 613 available records, 34 records were specific to the maintenance and repair of a facility falling under the three categories that this research is reviewing. These 34 records were identified through the use of the "find" function using the terms, "facility management," "maintenance," and "repair." The search results that yielded farming data were not utilized and are not included in the 34 records. The breakdown of the FACE reports coded in this research are presented in Table 12

Table 12: Breakdown of FACE Reports Utilized in this Research

\begin{tabular}{|c|c|c|c|}
\hline \# & Falls & Contact With/Struck By & Harmful Environments \\
\hline 1 & California Report 07CA007 & FACE 9717 In-House & California Report 11CA008 \\
\hline 2 & FACE Report No. 9013 & Oregon FACE Report 04OR003 & FACE Report No. 9104 \\
\hline 3 & Massachusetts 11-MA-008-01 & Oregon FACE Report 05OR008 & FACE Report No. 9014 \\
\hline 4 & FACE Report No. 9801 & California Report 00CA007 & FACE Report No. 8928 \\
\hline 5 & FACE Report No. 9104 & New York Report 02NY096 & Colorado Report 91CO074 \\
\hline 6 & Michigan Report No. 10MI006 & California Report 98CA004 & Oregon Report 04OR037 \\
\hline 7 & NY FACE Report 07NY080 & California Report 00CA009 & Alaska FACE Report 91-13 \\
\hline 8 & FACE Report 9621 & Virginia FACE Report No. 9239 & Washington 04WA080 \\
\hline 9 & Texas Report 98TX13301 & FACE Report No. 2002-05 & FACE Report No. 88-21 \\
\hline 10 & FACE Report 9506 & FACE Report No. 2006-02 & FACE Report No. 8610 \\
\hline 11 & & & California Report 06CA008 \\
\hline 12 & & & FACE Report 89-18 \\
\hline 13 & & & FACE Report 91-32 \\
\hline 14 & & & FACE Report 89-19 \\
\hline
\end{tabular}

Qualitative analysis of the FACE Reports used a color scheme in order to identify the applicable codes. For example, a yellow highlight indicates "Worker Details," while a purple highlight references a "Procedural Failure." In order to begin to identify patterns and correlate the data collected from the FACE Reports, an Excel spreadsheet was used for organizational purposes. During the organizational phase of the FACE Report data collection, an iterative process was used in order to identify the appropriate categories in the 
X-axis of the spreadsheet. As more FACE Reports were read and organized the nodes evolved. Figure 15 graphically presents the evolution of the X-axis nodes, while Figure 16 presents an image of the spreadsheet evolution.

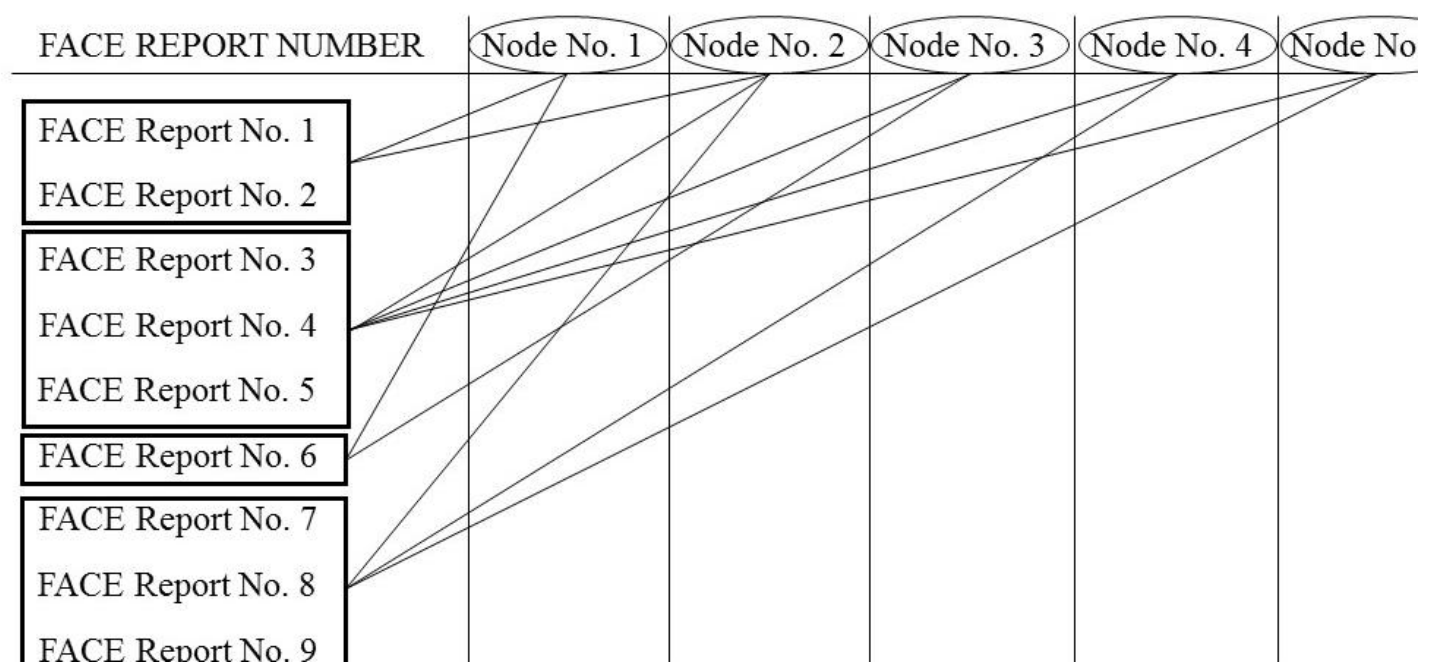

Figure 15: Evolution of the Main X-axis Nodes in the FACE Report Spreadsheet 


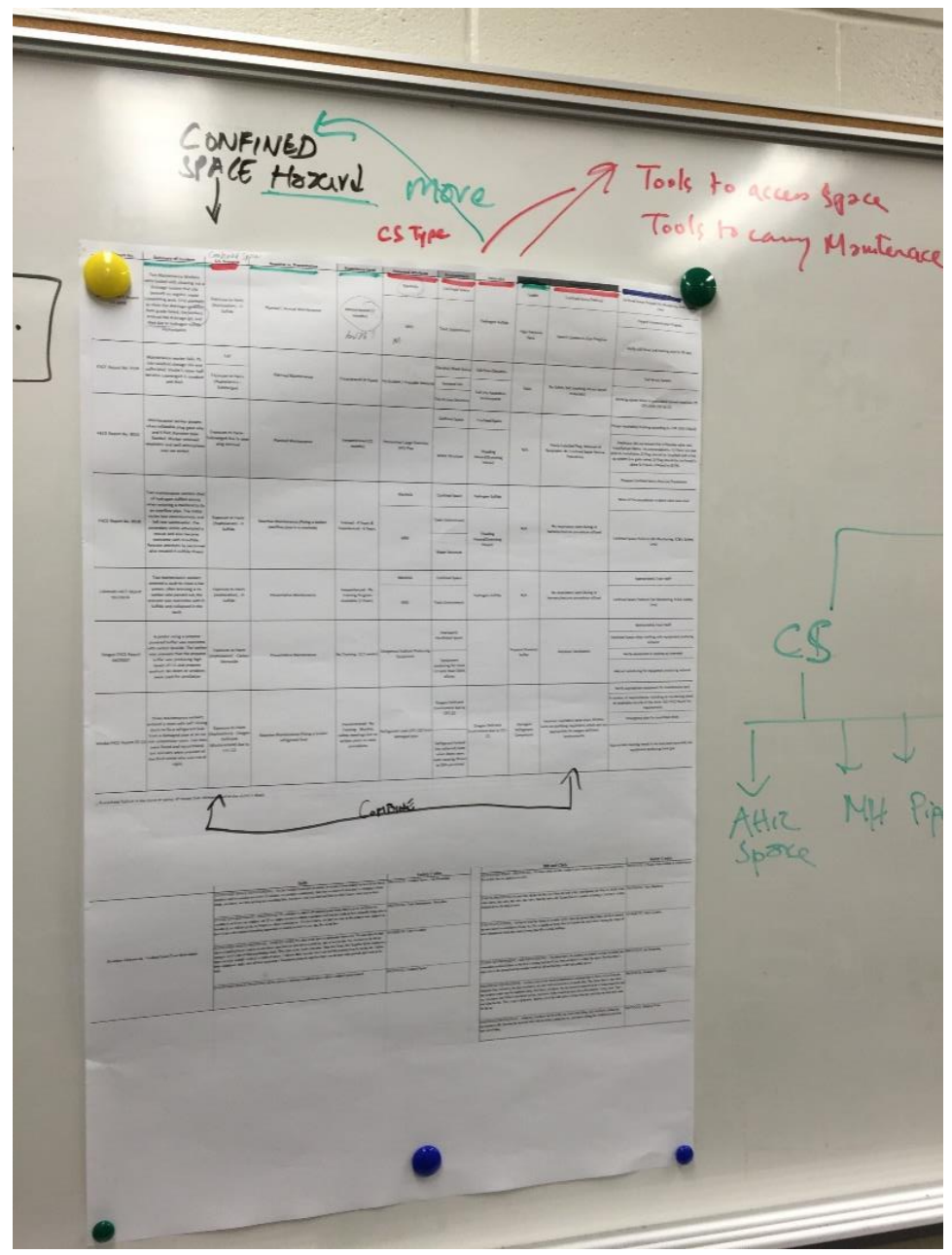

Figure 16: Image of the FACE Report Spreadsheet Evolution

Ultimately, the $\mathrm{X}$-axis nodes evolved into the following eleven categories:

1. FACE Report Identification Number

2. Summary of Incident

3. Tools to Carry Out Maintenance

4. Tools to Access Space

5. Reactive vs. Preventative Maintenance

6. Experience Level

7. Potential Attribute(s)

8. Environment

9. Hazard(s)

10. Procedural Failure(s)

11. Hazard Control(s) 
Figure 17 presents a single, contact with/struck by FACE Report regarding a maintenance electrician who was crushed to death when a limit switch was activated by the victim, who was leaning into the equipment (National Institute for Occupational Safety and Health 1997). In addition, the figure features how the information is organized within the Excel spreadsheet. The following is the summary as described in the FACE Report.

"On April 18, 1997, a 37-year-old male maintenance electrician (the victim died when his lower torso was crushed between the nip barrier (a wire-mesh gate) and the upper frame of a paper rewinder machine at a paper-manufacturing facility. Without first de-energizing, locking out, and tagging the machine, the victim began to replace the arm for the limit switch that controlled upward movement of the nip barrier. He climbed an 8-foot stepladder to access the top of the machine where the switch was located, and leaned into the 16-inch opening between the top of the nip barrier and the upper frame of the machine..."
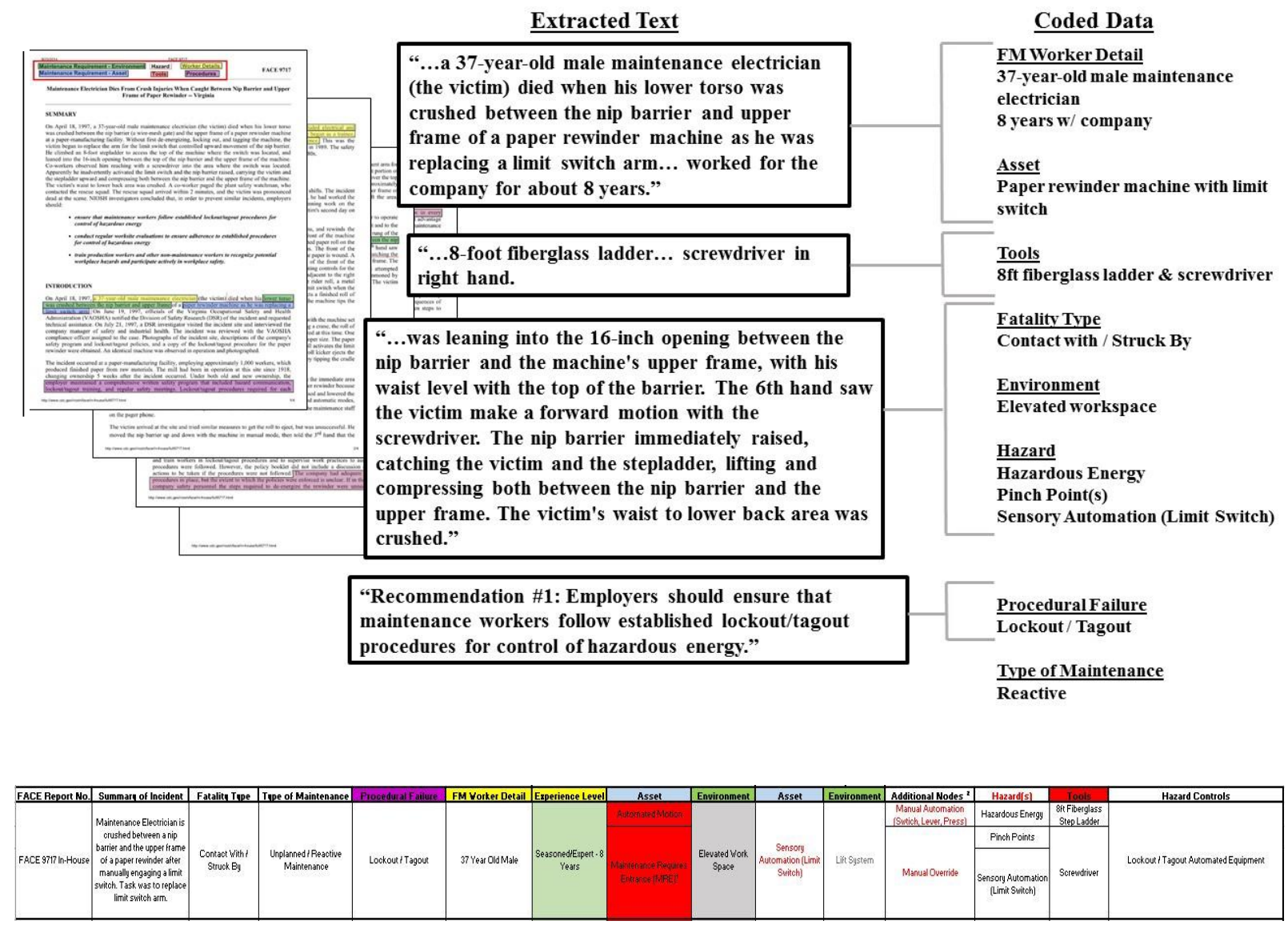

Figure 17: Coding Methodology of FACE Report - Example 
Based on the details provided from the FACE Report and the coded data, the following safety information can be extracted:

- $\quad$ Lockout / Tagout

- Hazardous Energy

- Pinch Points

- Automated Equipment (Limit Switch)

- $\quad$ Lift System (Ladder)

Additional analysis of the extracted safety data yields two types of information, safety input and safety protocol data. Safety input data are the inherent risks associated with a work activity, while safety protocol data are the mitigation techniques utilized in order to avoid the risk(s). To decipher which category the above attributes fall under, a knowledge category identifies preliminary links between the inputs and the protocols. These preliminary links will be greatly expanded upon during the Mind Mapping and DRPS development, but initially serve as an organizational step. Table 13 presents the safety attributes within the designated category for the above example. "Hazard Type" indicates which of the three hazard areas these attributes fall under:

- Harmful Substance and Environment - HARM

- Contact With / Struck By - CW/SB

- Falls - FALL

Table 13: Safety Attributes within the Designated Category - FACE Reports

\begin{tabular}{|c|c|c|c|}
\hline $\begin{array}{c}\text { Safety Input (Hazard } \\
\text { Type) }\end{array}$ & Knowledge & Safety Protocol & Knowledge \\
\hline $\begin{array}{c}\text { Hazardous Energy } \\
\text { (HARM) }\end{array}$ & $\begin{array}{c}\text { Relates to } \\
\text { Lockout/Tagout }\end{array}$ & Lockout / Tagout & $\begin{array}{l}\text { Relates to HARM and } \\
\text { CW/SB Inputs }\end{array}$ \\
\hline Pinch Points (CW/SB) & $\begin{array}{c}\text { Relates to } \\
\text { Lockout/Tagout }\end{array}$ & $\begin{array}{l}\text { Lift System- } \\
\text { Ladder }\end{array}$ & Relates to FALL Inputs \\
\hline $\begin{array}{l}\text { Automated Equipment- } \\
\text { Limit Switch (CW/SB) }\end{array}$ & $\begin{array}{c}\text { Relates to } \\
\text { Lockout/Tagout }\end{array}$ & & \\
\hline
\end{tabular}

By continuing the process of FACE report data collection and analysis for all 34 records, a comprehensive list of safety inputs and knowledge can be developed. Appendix 10.2-Appendix 10.5 presents all of the FACE Report data collection and analysis sheets. 


\subsection{Data Collection Interviews}

This research utilizes two types of interviews in order to obtain safety inputs and to validate the information developed from the three data collection instruments. These two types of interviews provide necessary trustworthiness in qualitative research. The interviews were conducted in the manner stated in Section 3.1.2 Research Methods and utilized purposive sampling, member checks, and thick descriptions. The first interview utilized was the Data Collection Interview, as described in Section 3.1.3.1 Data Collection \& Organization - Objective 1. The Data Collection Interview is used to identify safety inputs and protocols to be input into the framework. Table 14 presents the professional information for the six participants that were included in the Data Collection Interviews. Three data collection interviews took place, two in August 2015 and one in February 2016. In an effort to cover a wide range of expert opinions, participant job titles and responsibility range from upper management to skilled labor. As stated in Chapter 3, the participant's names and companies have been excluded from this list in order to maintain anonymity. All the names presented in Table $\mathbf{1 4}$ are randomly selected pseudonyms.

Table 14: Data Collection Interview Participant Information

\begin{tabular}{|c|c|c|c|c|c|}
\hline$\#$ & $\begin{array}{l}\text { Date } \\
\text { Interviewed }\end{array}$ & Pseudonym & Position & Company Type & Staff Size \\
\hline 1 & $8 / 11 / 15$ & Sally & $\begin{array}{l}\text { Safety Manager for } \\
\text { Facilities } \\
\text { Management }\end{array}$ & $\begin{array}{l}\text { Large University: } \\
\text { Southeast Region } \\
\text { USA }\end{array}$ & $500+$ \\
\hline \multirow[t]{2}{*}{2} & $8 / 13 / 15$ & Chris & $\begin{array}{l}\text { Director of Utilities } \\
\text { and Energy }\end{array}$ & $\begin{array}{l}\text { Large University: } \\
\text { Southeast Region } \\
\text { USA }\end{array}$ & $30+$ \\
\hline & $8 / 13 / 15$ & Bill & $\begin{array}{l}\text { Assistant Director of } \\
\text { Utilities and Energy }\end{array}$ & $\begin{array}{l}\text { Large University: } \\
\text { Southeast Region } \\
\text { USA }\end{array}$ & $30+$ \\
\hline \multirow{3}{*}{3} & 2/4/16 & Joe & Operations Manager & $\begin{array}{l}\text { Civic Center w/ } \\
\text { Arena \& Ice Rink } \\
\text { Southeast Region } \\
\text { USA }\end{array}$ & $100-150$ \\
\hline & $2 / 4 / 16$ & Dan & $\begin{array}{l}\text { Maintenance } \\
\text { Supervisor }\end{array}$ & $\begin{array}{l}\text { Civic Center w/ } \\
\text { Arena \& Ice Rink } \\
\text { Southeast Region } \\
\text { USA }\end{array}$ & $\sim 30$ \\
\hline & $2 / 4 / 16$ & Tim & Electrician & $\begin{array}{l}\text { Civic Center w/ } \\
\text { Arena \& Ice Rink } \\
\text { Southeast Region } \\
\text { USA }\end{array}$ & 2 \\
\hline
\end{tabular}


Upon completion of the interviews, the recordings were transcribed into a word document. This Word document was coded by hand and placed into a spreadsheet for organization and identification of applicable safety attributes. Along with demographic information about the interviewee, relevant quotes from the interview were segregated into six categories:

1. Falls

2. Hazardous Environment - Electrical

3. Hazardous Environment - Confined Space / Toxic Environment

4. Contact With / Struck By

5. Dual Processes (Statements that refer to more than one hazard in a single FM task)

6. Outside Factors to Safety - Environment

By aggregating full quotes into the six categories, applicable safety input and safety protocol data could then be extracted within the proper context. Figure 18 presents a sample of the coding methodology utilized for the Data Collection interviews and organization of the extracted text and coded data in an Excel Spreadsheet. 


\section{Extracted Text}

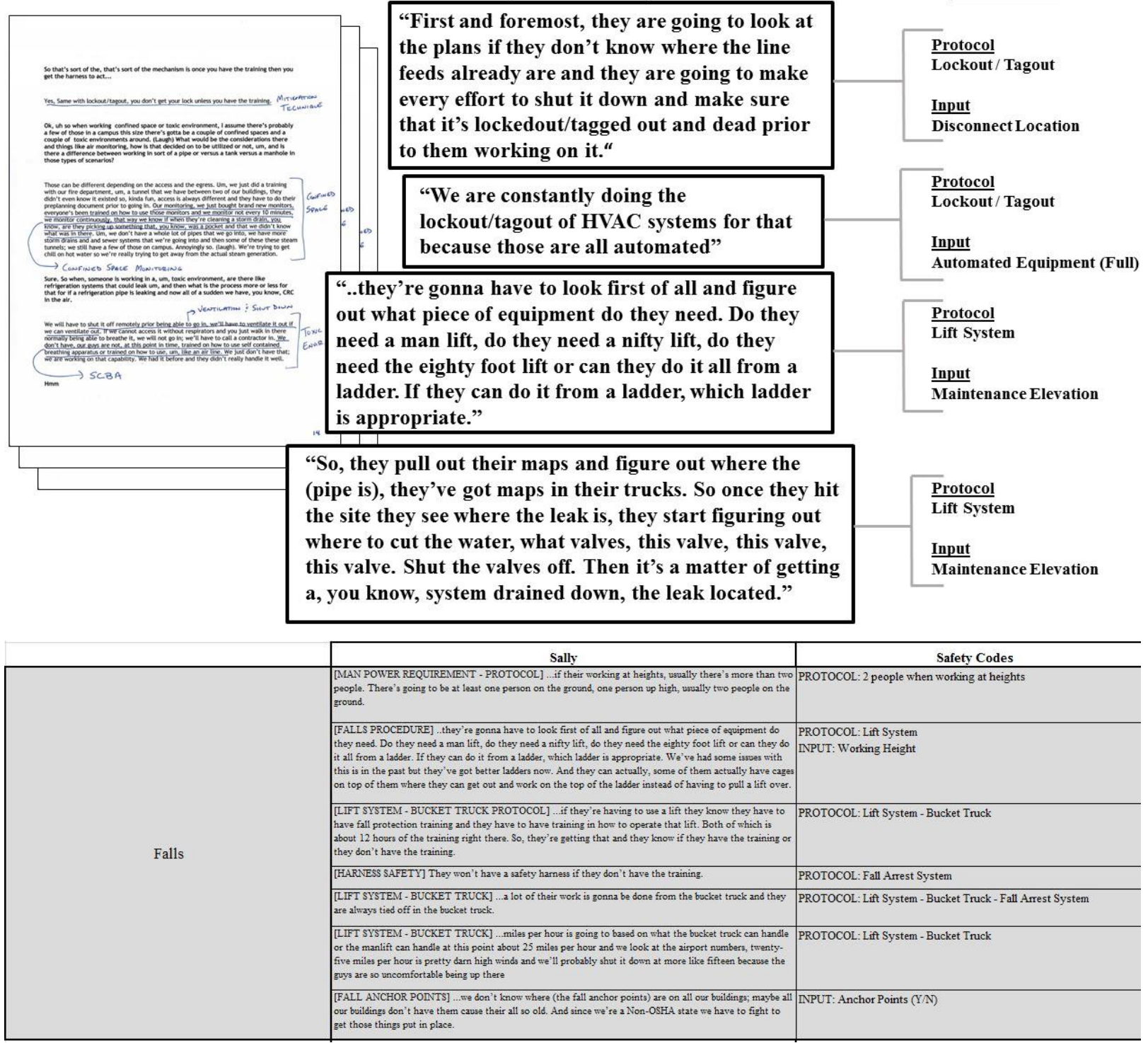

Figure 18: Coded Data Collection Interview and Organization - Example

Based on the above examples additional safety inputs and safety protocols can be extracted as presented in Table 15. In some instances, redundant inputs and protocols from the FACE Report and safety literature examples are identified. The redundancy within the data collection represents the confirmation of a data point between two or more sources, known as triangulation. 
Table 15: Safety Attributes within the Designated Category - Data Collection Interviews

\begin{tabular}{|c|c|}
\hline $\begin{array}{c}\text { Safety Input (Hazard } \\
\text { Type) }\end{array}$ & Knowledge \\
\hline $\begin{array}{l}\text { Disconnect Location } \\
\text { (HARM \& CW/SB) }\end{array}$ & $\begin{array}{c}\text { Relates to } \\
\text { Lockout/Tagout }\end{array}$ \\
\hline $\begin{array}{c}\text { Automated Equipment } \\
(\mathrm{CW} / \mathrm{SB})\end{array}$ & $\begin{array}{c}\text { Relates to } \\
\text { Lockout/Tagout }\end{array}$ \\
\hline $\begin{array}{c}\text { Maintenance Elevation } \\
\text { (FALL) }\end{array}$ & $\begin{array}{l}\text { Relates to Lift } \\
\text { Systems }\end{array}$ \\
\hline
\end{tabular}

\section{Safety Protocol Knowledge}

Lockout / Tagout

Relates to HARM and

Lift System-Man lift \& Ladder
CW/SB Inputs

Relates to FALL Inputs

The Data Collection interview questions and the data collection and analysis spreadsheets for the three interview transcripts are presented in Appendix 10.6-Appendix 10.9.

\subsection{Safety Literature}

Safety literature in the context of this research is any document that could provide insight into the safe maintenance of a facility applicable to the three hazard types that this research attempts to mitigate - falls, contact with / struck by, and exposure to harmful substances or environments. These documents could be a safety checklist, handbook, O\&M manual, OSHA pamphlet, etc. Review of the safety literature yielded seven categories used to code the information:

1. Hazard Type (Falls, Contact With / Struck By, Harmful Substances and Environments)

2. Source

3. Hazard

4. Hazard Definition

5. Safety Input

6. Safety Protocol

7. Protocol Requirements / Minimum Testing

Table 16 presents all of the safety information sources utilized in this research. In correlation with the other two sources of safety data, collection of safety inputs and protocols ceased when data saturation became apparent. 
Table 16: Safety Information Sources

Safety Information Data Collection Sources

\begin{tabular}{|l|l|}
\hline Environmental Protection Agency (2015) & United States Department of Labor (2007) \\
\hline Duke University (2012) FM Safety Sheet & United States Department of Labor (2011) \\
\hline Creighton University Facilities Management (2010) & Department of the Navy (2013) \\
\hline University of Minnesota Facilities Management (2008) & Coastal Carolina University (2003) \\
\hline Alberta Government (2014) & Office of Compliance (2010) \\
\hline
\end{tabular}

Figure 19 presents examples from Duke University (2012) and University of Maryland Facilities Management (2012) of the type of safety literature utilized in order to obtain additional safety inputs and safety protocols and how the data was organized within the Excel Spreadsheet.

\section{Extracted Text}

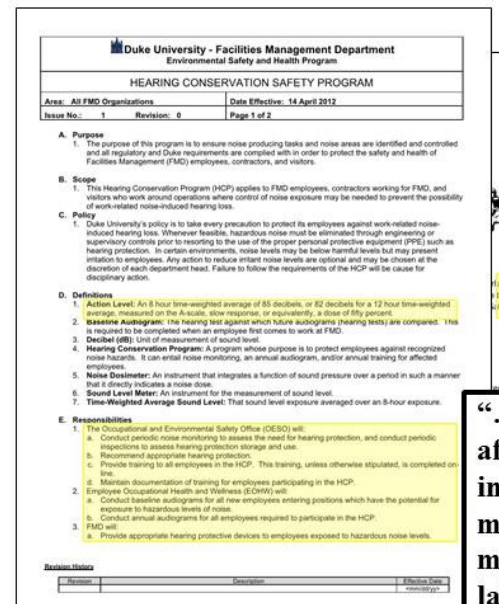
laboratory hazard signs.
"An 8 hour time-weighted average of 85 decibels, or 82 decibels for a 12 hour timeweighted average, measure on the $A$-scale, slow response, or equivalently, a dose of fifty percent."

3
Coded Data

Protocol

Hearing Protection

Input

Environmental Decibel Level

Protocol

Noise Monitoring

assess the need for hearing protection..."

"...Employees learn how substances enter and affect the body, how to maintain a chemical inventory for their area, how to obtain and read material safety data sheets (MSDS), how to maintain required labeling, and how to read

\begin{tabular}{|c|c|c|c|c|c|c|}
\hline Hazard Type & Source & Hazard & Hazard Definition & Safety Input & Safety Protocol & Protocol Requiremets / Minimum Testing \\
\hline \multirow[t]{2}{*}{ Confined Space } & \multirow[t]{2}{*}{$\begin{array}{c}\text { Duke University } \\
\text { Facilities Management }\end{array}$} & \multirow[t]{2}{*}{ Hearing Loss } & \multirow{2}{*}{$\begin{array}{l}\text { Workers exposed to } 85 \mathrm{dBA} \text { or above } \\
\text { time-weighted average for an } 8 \text { hour } \\
\text { period or } 82 \mathrm{dBA} \text { for a } 12 \text { hour time- } \\
\text { weighted average, measured on the } \mathrm{A} \text { - } \\
\text { scale, slow response, or equivalently, a } \\
\text { dose of } 50 \%\end{array}$} & \multirow[t]{2}{*}{$\begin{array}{l}\text { Environmental } \\
\text { Decibel Level }\end{array}$} & Noise Dosimeter & $\begin{array}{c}\text { An instrucment that integrates a function } \\
\text { of sound pressure over a period in such a } \\
\text { manner that it directly indicates a noise } \\
\text { dose }\end{array}$ \\
\hline & & & & & Sound Level Meter & $\begin{array}{l}\text { An instrument for the measurement of } \\
\text { sound level }\end{array}$ \\
\hline
\end{tabular}

Figure 19: Coded Safety Literature and Organization - Example

From the above safety literature examples we can identify additional safety inputs and safety protocols applicable to the three safety hazards that this research is evaluating. Table 17 presents the extracted data from the examples. 
Table 17: Safety Attributes within the Designated Category - Safety Literature

\begin{tabular}{|c|c|}
\hline $\begin{array}{c}\text { Safety Input (Hazard } \\
\text { Type) }\end{array}$ & Knowledge \\
\hline $\begin{array}{c}\text { Environment Decibel } \\
\text { Level (HARM) }\end{array}$ & $\begin{array}{c}\text { Relates to } \\
\text { Hearing } \\
\text { Protection \& } \\
\text { Noise Mon. }\end{array}$ \\
\hline $\begin{array}{c}\text { Chemical Environment } \\
\text { (HARM) }\end{array}$ & $\begin{array}{c}\text { Relates to MSDS } \\
\text { Protocols }\end{array}$ \\
\hline
\end{tabular}

\begin{tabular}{|c|c|}
\hline Safety Protocol & Knowledge \\
\hline Hearing Protection & $\begin{array}{c}\text { Relates to HARM } \\
\text { Inputs }\end{array}$ \\
\hline SDS Protocol & $\begin{array}{c}\text { Relates to HARM } \\
\text { Inputs }\end{array}$ \\
\hline Noise Monitoring & $\begin{array}{c}\text { Relates to HARM } \\
\text { Inputs }\end{array}$ \\
\hline
\end{tabular}

Appendix 10.10 presents the safety information data collection and analysis spreadsheet.

\subsection{Data Categorization and Mind Mapping}

Upon reaching data saturation, the acquired safety data from the three methods of data collection is comprehensively coded, renamed, and categorized into the three specific hazard areas that this research is attempting to mitigate. The identified FM hazards, known as safety inputs, are associated with applicable hazard mitigation techniques (safety protocols), known as knowledge. In addition, production information for the safety inputs, known as data sourcing, is identified. Production encompasses information relating to the identification of the phase of the building lifecycle the information is produced, who is typically responsible for the creation of the asset or area causing the hazard, and what method of data transfer is likely for that information. Data sourcing information was correlated through constructivist theory and is a result of author experience. Using the data sourcing information in correlation with the safety inputs and associated protocols creates the backbone for the data loading and transfer phases addressed in Chapter 5. Table 18 presents a partial example of the initial categorization of the HARM safety inputs and protocols.

In order to further organize the data, the safety inputs and protocols is placed into mind maps. Figure 20 presents an example Mind Map of the Contact With/Struck By (CW/SB) category. In order to develop the Mind Maps, the safety inputs and protocols were further structured by an inherent hierarchy. For example, "Hazardous Energy" is identified through the data collection as a safety input requiring mitigation during an FM task. In order to mitigate "Hazardous Energy," a FM worker would need to acquire additional information regarding the hazardous energy, such as "Voltage", "Disconnect Location", etc. Structuring the safety information into mind maps aids in the establishment of the Asset Safety Identification Tool (Chapter 5) and the Data Retrieval and Processing System (Chapter 6), while secondarily acting as a 
graphical tool for industry professionals to use during the data validation interviews presented in Section 4.5 Data Validation.

Table 18: Initial Categorization Safety Inputs, Data Sourcing, and Protocols (Partial)

\section{HARMFUL ENVIRONMENTS \& SUBSTANCES (HARM)}

\begin{tabular}{|c|c|c|c|c|}
\hline \multicolumn{5}{|c|}{ DATA SOURCING } \\
\hline Safety Input & $\begin{array}{l}\text { Typical } \\
\text { Production } \\
\text { Phase }\end{array}$ & $\begin{array}{l}\text { Primary } \\
\text { Developer }\end{array}$ & $\begin{array}{l}\text { Method of } \\
\text { Transfer }\end{array}$ & $\begin{array}{l}\text { Knowledge: Relates } \\
\text { to Safety Protocol }\end{array}$ \\
\hline \multirow{4}{*}{ Hazardous Energy } & \multirow{4}{*}{ Design } & \multirow{4}{*}{$\mathrm{A} / \mathrm{E}$} & \multirow{4}{*}{$\begin{array}{c}\text { Native BIM } \\
\text { Model }\end{array}$} & Lockout/Tagout \\
\hline & & & & Hot Sticks/Metering \\
\hline & & & & Permits \\
\hline & & & & Arc Flash Protection \\
\hline $\begin{array}{l}\text { Elec. Disconnect } \\
\text { Location(s) }\end{array}$ & Design & $\mathrm{A} / \mathrm{E}$ & $\begin{array}{l}\text { Native BIM } \\
\text { Model }\end{array}$ & Lockout/Tagout \\
\hline \multirow{4}{*}{ Voltage } & \multirow{4}{*}{ Design } & \multirow{4}{*}{$\mathrm{A} / \mathrm{E}$} & \multirow{4}{*}{$\begin{array}{l}\text { Native BIM } \\
\text { Model }\end{array}$} & Lockout/Tagout \\
\hline & & & & Arc Flash Protection \\
\hline & & & & Permits \\
\hline & & & & Hot Sticks/Metering \\
\hline
\end{tabular}




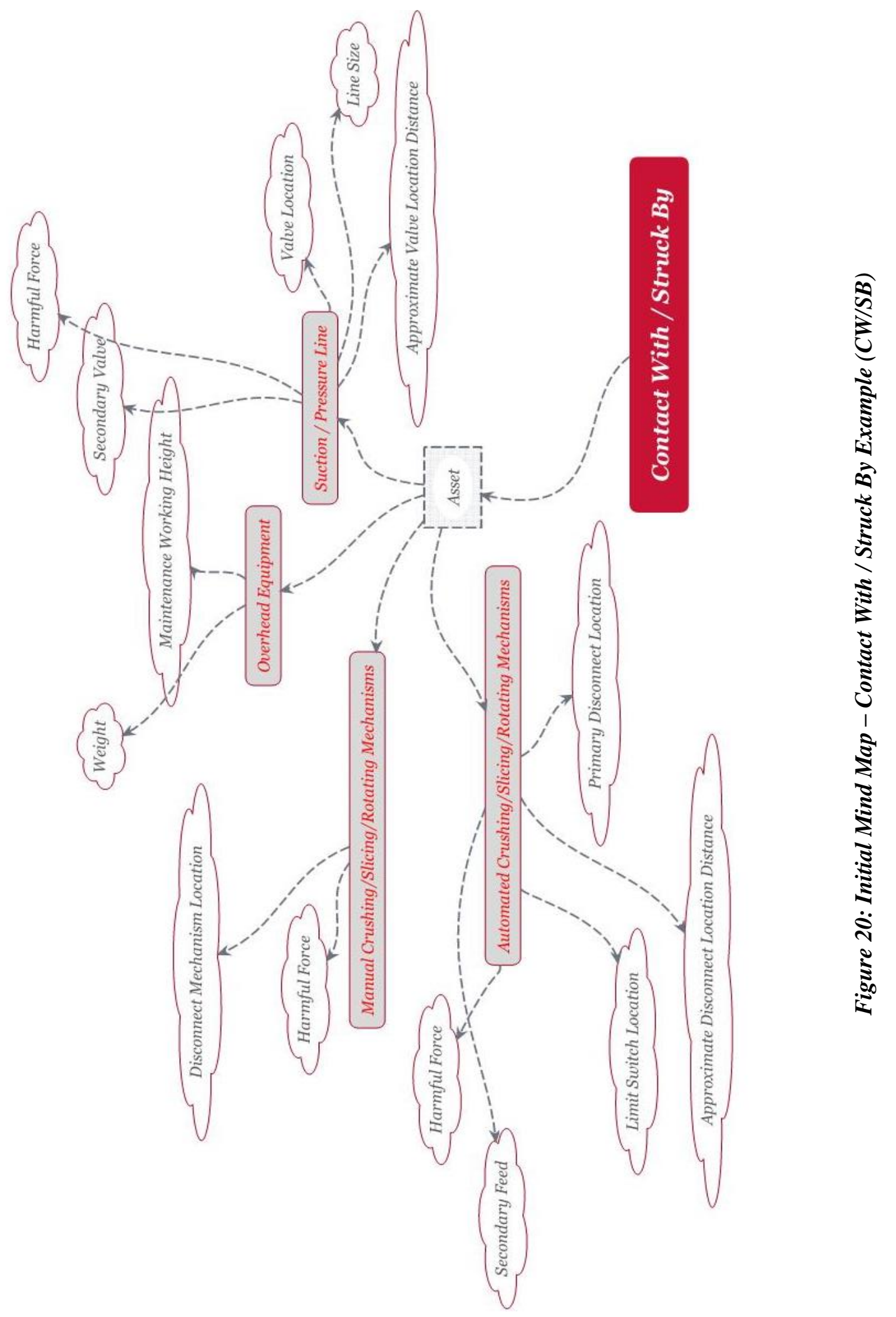




\subsection{Data Validation}

In order to identify a comprehensive list of safety inputs, protocols, and typical data sourcing, industry professionals are utilized to validate the collected data. This is executed through the Data Validation interview as presented in Section 3.1.3.1 Data Collection \& Organization-Objective 1. Table 19 presents the participants that were utilized for the Data Validation interviews.

\section{Table 19: Data Validation Interview Participants}

\begin{tabular}{|l|l|l|l|}
\hline Date Interviewed & Pseudonym & Position & \multicolumn{2}{|l|}{ Company Type } \\
\hline $6 / 17 / 16$ & Ryan & Facilities Safety Inspector & $\begin{array}{l}\text { Large University: East } \\
\text { Region USA }\end{array}$ \\
\hline $6 / 21 / 16$ & Sally & $\begin{array}{l}\text { Safety Manager for Facilities } \\
\text { Management }\end{array}$ & $\begin{array}{l}\text { Large University: Southeast } \\
\text { Region USA }\end{array}$ \\
\hline
\end{tabular}

Utilizing triangulation through the three methods of data collection and the use of Data Validation interviews for the validation of the collected data, the developed safety inputs and protocols can now be utilized by the safety framework. The complete list of the 28 safety inputs, associated knowledge, and sourcing is presented in Table 20. Appendix 10.12 Data Validation Interview Data Adjustments / Additions presents the initial data inputs, sourcing, and protocols with the variations made to the list through the Data Validation interviews.

Table 20: Safety Inputs and Protocols

HARMFUL ENVIRONMENTS \& SUBSTANCES (HARM)

\begin{tabular}{|c|c|c|c|c|}
\hline \multicolumn{5}{|c|}{ DATA SOURCING } \\
\hline Safety Input & $\begin{array}{l}\text { Typical } \\
\text { Production } \\
\text { Phase }\end{array}$ & $\begin{array}{l}\text { Primary } \\
\text { Developer }\end{array}$ & $\begin{array}{l}\text { Method of } \\
\text { Transfer }\end{array}$ & $\begin{array}{l}\text { Knowledge: Relates to } \\
\text { Safety Protocol }\end{array}$ \\
\hline \multirow{4}{*}{ Hazardous Energy } & \multirow{4}{*}{ Design } & \multirow{4}{*}{$\mathrm{A} / \mathrm{E}$} & \multirow{4}{*}{$\begin{array}{l}\text { Native BIM } \\
\text { Model }\end{array}$} & Lockout/Tagout \\
\hline & & & & Hot Sticks/Metering \\
\hline & & & & Permits \\
\hline & & & & Arc Flash Protection \\
\hline Elec. Disconnect Location(s) & Design & $\mathrm{A} / \mathrm{E}$ & $\begin{array}{l}\text { Native BIM } \\
\text { Model }\end{array}$ & Lockout/Tagout \\
\hline \multirow{4}{*}{ Voltage } & \multirow{4}{*}{ Design } & \multirow{4}{*}{$\mathrm{A} / \mathrm{E}$} & \multirow{4}{*}{$\begin{array}{l}\text { Native BIM } \\
\text { Model }\end{array}$} & Lockout/Tagout \\
\hline & & & & Arc Flash Protection \\
\hline & & & & Permits \\
\hline & & & & Hot Sticks/Metering \\
\hline Approx. Disconnect Distance & Design & $\mathrm{A} / \mathrm{E}$ & $\begin{array}{l}\text { Native BIM } \\
\text { Model }\end{array}$ & Lockout/Tagout \\
\hline
\end{tabular}




\begin{tabular}{|c|c|c|c|c|}
\hline \multirow{2}{*}{ Asbestos Potential } & \multirow{2}{*}{ FM } & \multirow{2}{*}{ FM Staff } & \multirow{2}{*}{ Direct Input } & Asbestos Management \\
\hline & & & & Respiratory Protection \\
\hline \multirow{2}{*}{ Environment Decibel Level } & \multirow{2}{*}{ Construction } & \multirow{2}{*}{$\begin{array}{c}\text { Manufacturer } \\
/ \\
\text { Subcontractor }\end{array}$} & \multirow{2}{*}{ Non-3D } & $\begin{array}{c}\text { Hearing Protection / Noise } \\
\text { Dampening }\end{array}$ \\
\hline & & & & Noise Monitoring \\
\hline \multirow{8}{*}{ Environmental Air Quality } & \multirow{8}{*}{ Design } & \multirow{8}{*}{$\mathrm{A} / \mathrm{E}$} & \multirow{8}{*}{$\begin{array}{l}\text { Native BIM } \\
\text { Model }\end{array}$} & $\begin{array}{c}\text { SDS / Chemical } \\
\text { Management }\end{array}$ \\
\hline & & & & Respiratory Protection \\
\hline & & & & Refrigerant Management \\
\hline & & & & PCB Management \\
\hline & & & & Silica Management \\
\hline & & & & Asbestos Management \\
\hline & & & & Lead Management \\
\hline & & & & Permits \\
\hline \multirow{9}{*}{$\begin{array}{c}\text { Manhole / Tank / Confined } \\
\text { Space }\end{array}$} & \multirow{9}{*}{ Design } & \multirow{9}{*}{$\mathrm{A} / \mathrm{E}$} & \multirow{9}{*}{$\begin{array}{l}\text { Native BIM } \\
\text { Model }\end{array}$} & $\begin{array}{c}\text { Hearing Protection / Noise } \\
\text { Dampening }\end{array}$ \\
\hline & & & & Noise Monitoring \\
\hline & & & & $\begin{array}{c}\text { SDS / Chemical } \\
\text { Management }\end{array}$ \\
\hline & & & & Air Monitoring \\
\hline & & & & Temperature Monitoring \\
\hline & & & & Valve Location(s) \\
\hline & & & & Confined Space Protocol \\
\hline & & & & Permits \\
\hline & & & & Ventilation \\
\hline \multirow{4}{*}{$\begin{array}{l}\text { Oxygen Deficient / Oxygen } \\
\text { Enriched / Carbon Monoxide } \\
\text { Environment }\end{array}$} & \multirow{4}{*}{ Design } & \multirow{4}{*}{$\mathrm{A} / \mathrm{E}$} & \multirow{4}{*}{$\begin{array}{l}\text { Native BIM } \\
\text { Model }\end{array}$} & Permits \\
\hline & & & & Air Monitoring \\
\hline & & & & Ventilation \\
\hline & & & & Respiratory Protection \\
\hline \multirow{3}{*}{ Particulate Environment } & \multirow{3}{*}{ Design } & \multirow{3}{*}{$\mathrm{A} / \mathrm{E}$} & \multirow{3}{*}{$\begin{array}{l}\text { Native BIM } \\
\text { Model }\end{array}$} & Air Monitoring \\
\hline & & & & Ventilation \\
\hline & & & & Respiratory Protection \\
\hline Exhaust Producing Asset & FM & FM Staff & Direct Input & Ventilation \\
\hline \multirow{3}{*}{ Flooding Potential } & \multirow{3}{*}{ Design } & \multirow{3}{*}{$\mathrm{A} / \mathrm{E}$} & \multirow{3}{*}{$\begin{array}{l}\text { Native BIM } \\
\text { Model }\end{array}$} & Inflatable Valve \\
\hline & & & & Valve Location \\
\hline & & & & Safety Line \\
\hline Hazardous Asset Temperature & Design & $\mathrm{A} / \mathrm{E}$ & $\begin{array}{l}\text { Native BIM } \\
\text { Model }\end{array}$ & $\begin{array}{c}\text { Burn Mitigation / Frostbite } \\
\text { Mitigation / Hypo } \\
\text { (Hyper)thermia }\end{array}$ \\
\hline
\end{tabular}




\begin{tabular}{|c|c|c|c|c|}
\hline \multirow[t]{2}{*}{ Outdoor Environment } & \multirow[t]{2}{*}{ Design } & \multirow[t]{2}{*}{$\mathrm{A} / \mathrm{E}$} & \multirow{2}{*}{$\begin{array}{l}\text { Native BIM } \\
\text { Model }\end{array}$} & $\begin{array}{c}\text { Weather Considerations - } \\
\text { Heat, Cold, Precipitation, } \\
\text { Wind }\end{array}$ \\
\hline & & & & $\begin{array}{c}\text { Nature - Animals, } \\
\text { Allergens, Plant Life }\end{array}$ \\
\hline \multirow{2}{*}{ Lead Potential } & \multirow{2}{*}{ FM } & \multirow{2}{*}{ FM Staff } & \multirow{2}{*}{ Direct Input } & Lead Management \\
\hline & & & & Respiratory Protection \\
\hline \multirow{6}{*}{$\begin{array}{l}\text { Hazardous Chemical } \\
\text { Production / Storage }\end{array}$} & \multirow{6}{*}{ Design } & \multirow{6}{*}{$\mathrm{A} / \mathrm{E}$} & \multirow{6}{*}{$\begin{array}{l}\text { Native BIM } \\
\text { Model }\end{array}$} & $\begin{array}{c}\text { SDS / Chemical } \\
\text { Management }\end{array}$ \\
\hline & & & & Air Monitoring \\
\hline & & & & Secondary Containment \\
\hline & & & & Ventilation \\
\hline & & & & Respiratory Protection \\
\hline & & & & Valve Location \\
\hline \multirow{2}{*}{ Radiation } & \multirow{2}{*}{ Design } & \multirow{2}{*}{$\mathrm{A} / \mathrm{E}$} & \multirow{2}{*}{$\begin{array}{l}\text { Native BIM } \\
\text { Model }\end{array}$} & Monitoring \\
\hline & & & & Radiation Management \\
\hline \multirow{2}{*}{$\begin{array}{l}\text { Polychlorinated Biphenyls } \\
\text { (PCB) Potential }\end{array}$} & \multirow{2}{*}{ FM } & \multirow{2}{*}{ FM Staff } & \multirow{2}{*}{ Direct Input } & PCB Management \\
\hline & & & & Respiratory Protection \\
\hline
\end{tabular}

FALLS (FALL)

\begin{tabular}{|c|c|c|c|c|}
\hline \multicolumn{5}{|c|}{ DATA SOURCING } \\
\hline Safety Input & $\begin{array}{l}\text { Typical } \\
\text { Production } \\
\text { Phase }\end{array}$ & $\begin{array}{l}\text { Primary } \\
\text { Developer }\end{array}$ & $\begin{array}{l}\text { Method of } \\
\text { Transfer }\end{array}$ & $\begin{array}{l}\text { Knowledge: Relates to } \\
\text { Safety Protocol }\end{array}$ \\
\hline \multirow{3}{*}{$\begin{array}{c}\text { Maintenance Requires Lift } \\
\text { System }\end{array}$} & \multirow{3}{*}{ All } & \multirow{3}{*}{ All } & \multirow{3}{*}{$\begin{array}{l}\text { Native BIM } \\
\text { Model, Non- } \\
\text { 3D, or Direct } \\
\text { Input }\end{array}$} & Lift System \\
\hline & & & & Working Height \\
\hline & & & & $\begin{array}{c}\text { Fall Arrest System / } \\
\text { Anchorage }\end{array}$ \\
\hline \multirow{5}{*}{ Roof / Deck } & \multirow{5}{*}{ Design } & \multirow{5}{*}{$\mathrm{A} / \mathrm{E}$} & \multirow{5}{*}{$\begin{array}{l}\text { Native BIM } \\
\text { Model }\end{array}$} & Lift System \\
\hline & & & & $\begin{array}{l}\text { Chemical Venting } \\
\text { (Industrial Hygiene) }\end{array}$ \\
\hline & & & & $\begin{array}{c}\text { Fall Arrest System / } \\
\text { Anchorage }\end{array}$ \\
\hline & & & & Barricading / Notification \\
\hline & & & & Outdoor Environment \\
\hline \multirow{4}{*}{ Ledge / Hole } & \multirow{4}{*}{ Design } & \multirow{4}{*}{$\mathrm{A} / \mathrm{E}$} & \multirow{4}{*}{$\begin{array}{l}\text { Native BIM } \\
\text { Model }\end{array}$} & Lift System \\
\hline & & & & $\begin{array}{c}\text { Fall Arrest System / } \\
\text { Anchorage }\end{array}$ \\
\hline & & & & Safety Line \\
\hline & & & & Barricading / Notification \\
\hline
\end{tabular}




\section{CONTACT WITH / STRUCK BY (CW/SB)}

\begin{tabular}{|c|c|c|c|c|}
\hline \multicolumn{4}{|c|}{ DATA SOURCING } & \multirow[b]{2}{*}{$\begin{array}{l}\text { Knowledge: Relates to } \\
\text { Safety Protocol }\end{array}$} \\
\hline Safety Input & $\begin{array}{l}\text { Typical } \\
\text { Production } \\
\text { Phase }\end{array}$ & $\begin{array}{l}\text { Primary } \\
\text { Developer }\end{array}$ & $\begin{array}{l}\text { Method of } \\
\text { Transfer }\end{array}$ & \\
\hline \multirow{2}{*}{$\begin{array}{c}\text { Manual } \\
\text { Crushing/Rotating/Slicing } \\
\text { Mechanism }\end{array}$} & \multirow{2}{*}{ Construction } & \multirow{2}{*}{$\begin{array}{c}\text { Manufacturer } \\
/ \\
\text { Subcontractor }\end{array}$} & \multirow{2}{*}{$\begin{array}{l}\text { Native BIM } \\
\text { Model or } \\
\text { Non-3D }\end{array}$} & Lockout/Tagout \\
\hline & & & & Barricading / Notification \\
\hline \multirow{4}{*}{$\begin{array}{c}\text { Automated } \\
\text { Crushing/Rotating/Slicing } \\
\text { Mechanism }\end{array}$} & \multirow{4}{*}{ Construction } & \multirow{4}{*}{$\begin{array}{l}\text { Manufacturer } \\
\text { / } \\
\text { Subcontractor }\end{array}$} & \multirow{4}{*}{$\begin{array}{l}\text { Native BIM } \\
\text { Model or } \\
\text { Non-3D }\end{array}$} & Lockout/Tagout \\
\hline & & & & Hot Sticks/Metering \\
\hline & & & & Permits \\
\hline & & & & Barricading / Notification \\
\hline \multirow{4}{*}{ Suction / Pressure Line } & \multirow{4}{*}{ Design } & \multirow{4}{*}{$\mathrm{A} / \mathrm{E}$} & \multirow{4}{*}{$\begin{array}{l}\text { Native BIM } \\
\text { Model }\end{array}$} & Safety Line \\
\hline & & & & $\begin{array}{c}\text { Burn Mitigation / Frostbite } \\
\text { Mitigation / Hypo } \\
\text { (Hyper)thermia }\end{array}$ \\
\hline & & & & Valve Location(s) \\
\hline & & & & Barricading / Notification \\
\hline Valve Location(s) & Design & $\mathrm{A} / \mathrm{E}$ & $\begin{array}{c}\text { Native BIM } \\
\text { Model } \\
\end{array}$ & Line Isolation \\
\hline \multirow{4}{*}{ Overhead Equipment } & \multirow{4}{*}{ All } & \multirow{4}{*}{ All } & \multirow{4}{*}{$\begin{array}{l}\text { Native BIM } \\
\text { Model, Non- } \\
\text { 3D, or Direct } \\
\text { Input }\end{array}$} & Barricading / Notification \\
\hline & & & & Approach Vector \\
\hline & & & & Support Structure \\
\hline & & & & Equipment Weight \\
\hline $\begin{array}{c}\text { Approx. Valve Location } \\
\text { Distances } \\
\end{array}$ & Design & $\mathrm{A} / \mathrm{E}$ & $\begin{array}{c}\text { Native BIM } \\
\text { Model } \\
\end{array}$ & Line Isolation \\
\hline Asset Support Structure & Design & $\mathrm{A} / \mathrm{E}$ & $\begin{array}{c}\text { Native BIM } \\
\text { Model }\end{array}$ & Approach Vector \\
\hline
\end{tabular}

Mind Mapping is utilized to further structure the safety information. By organizing and categorizing (analyzing) the relevant safety properties, a hierarchy of information can be established. This hierarchy will aid in the rule and relationship development during the Analyze Phase of this research. Figures 21, 22, \& 23 present the hierarchical Mind Mapping of the three hazard types, utilizing the safety inputs and protocols in Table 20. 


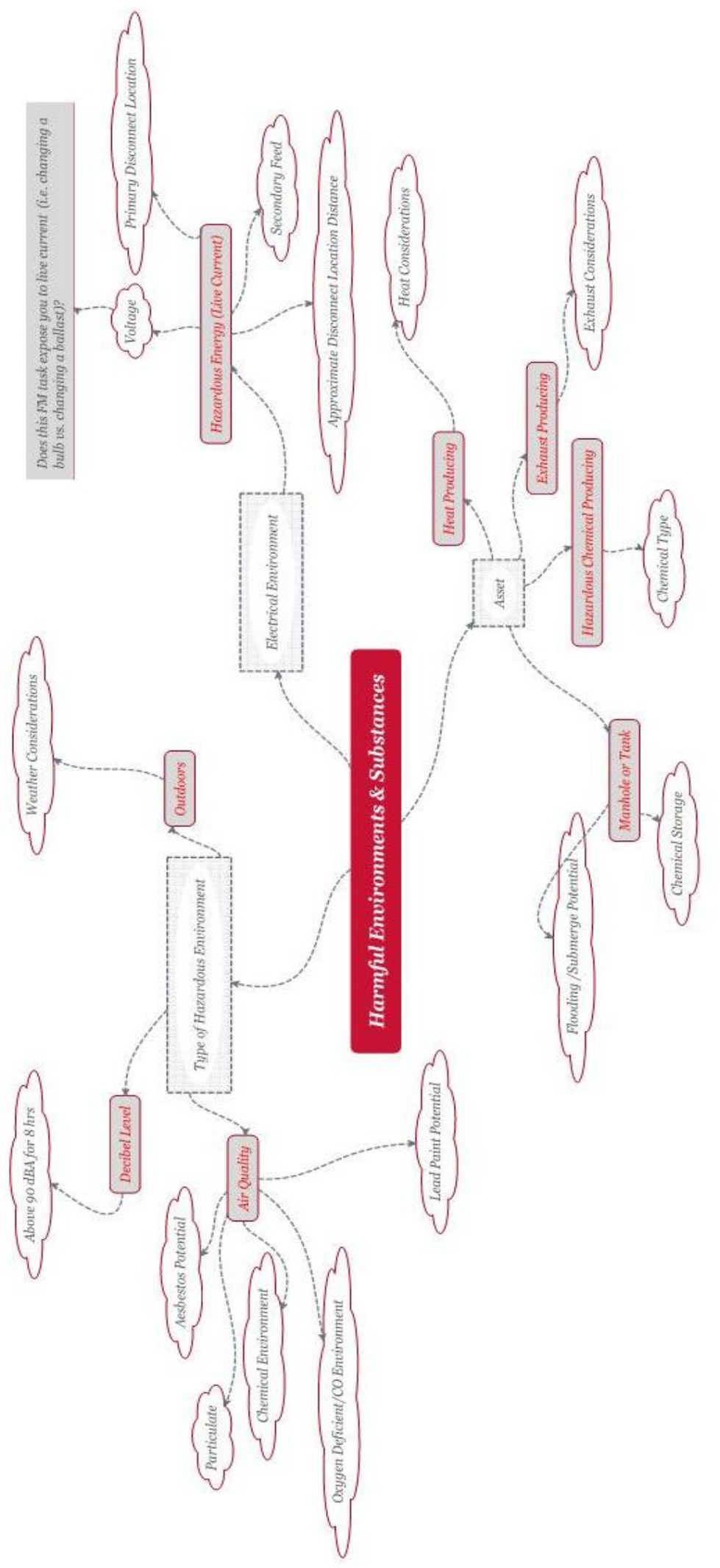

ปิ) 

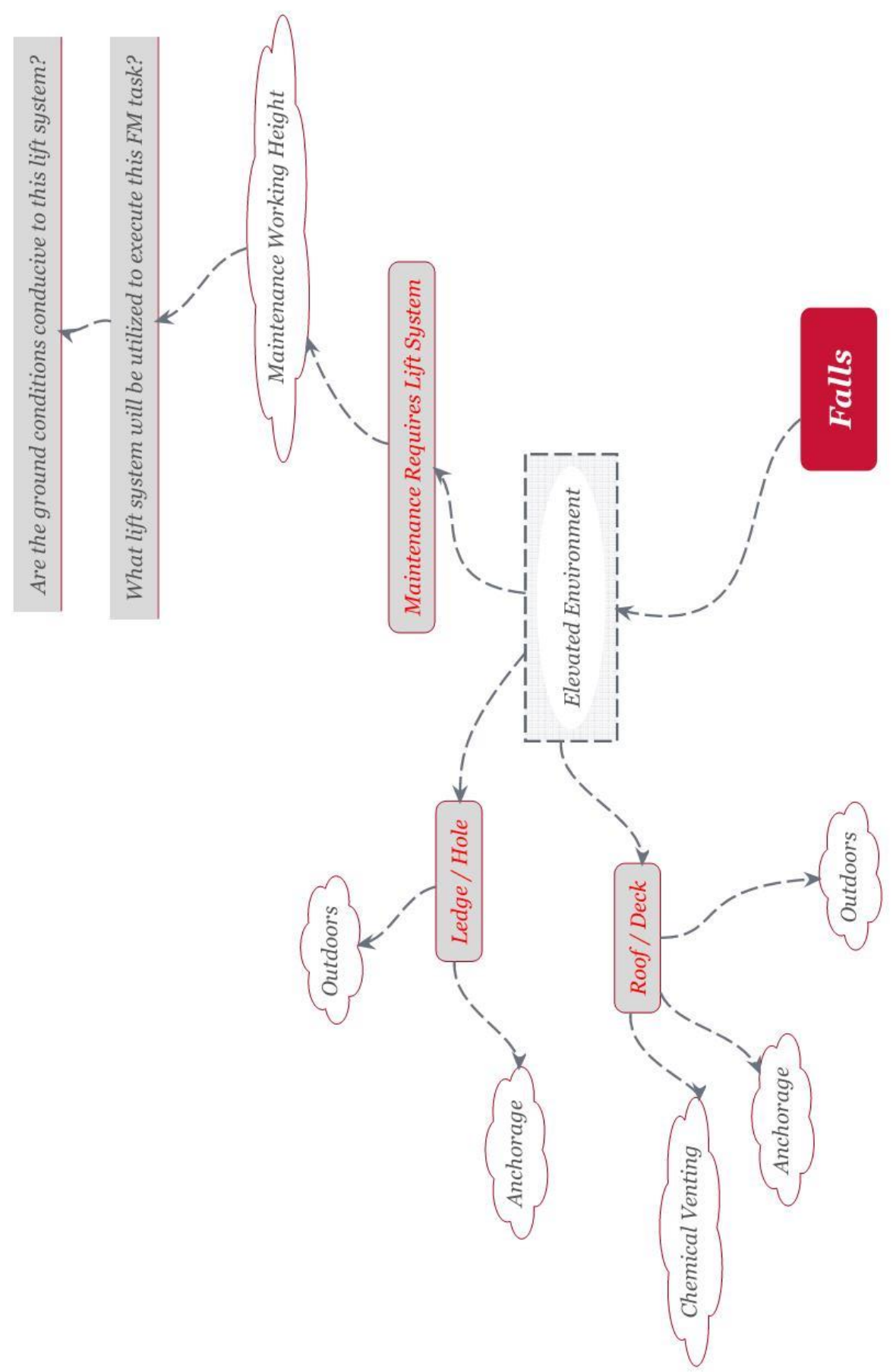

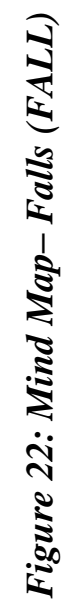




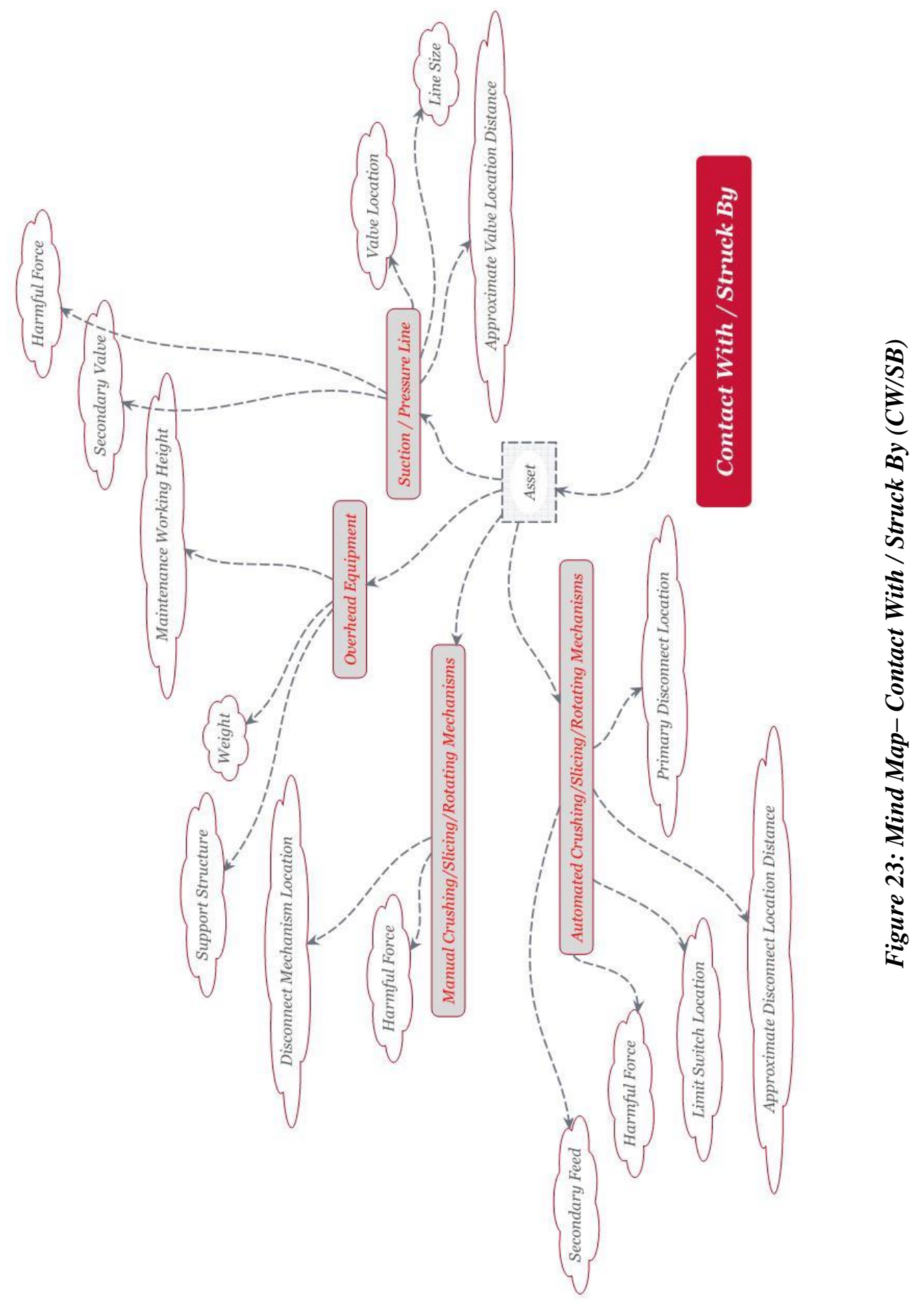




\subsection{Fundamental Safety Protocols}

This chapter has identified the safety inputs, data sourcing, and associated protocols required to develop a DRPS that will present task-specific safety information to FM workers prior to a FM task. The information is intended to mitigate the hazard areas that this research addresses; however, during this data collection phase, three "Fundamental Safety Protocols" arose regardless of the hazard being mitigated or the task being executed. These three protocols transcend the task-specific nature of each task and apply in every scenario. Because these assumptions are required in every scenario regardless of hazard type being mitigated, they are not presented in Table 20. However, these inputs will always be presented in the protocol sheet produced by the DRPS (Chapter 6) regardless of the FM task being executed. They are as follows:

- PPE - A FM worker must be aware of the standard PPE requirements for executing maintenance tasks as required by OSHA or the facility of employment.

- Worker Qualifications - A FM worker must not attempt tasks that they are not qualified to execute. Research has shown that a portion of workers will attempt tasks that they have not been formally trained to conduct.

- Condition Assessment - Prior to the start of any FM task, a condition assessment of the asset requiring maintenance, the environment, and the tools being utilized must be completed. By nature, repair and maintenance work is needed when assets malfunction or break, potentially creating a hazard that could not be foreseen. For example, a broken pipe has created a sharp or serrated edge. This type of hazard is not a condition of the asset or environment, but of the energy causing the asset malfunction. Mitigation of this hazard could require additional tools, PPE, or approach vectors, but ultimately would be at the worker's discretion.

\subsection{Data Collection Conclusions}

Completion of the Measure Phase, using data validation through Data Validation interviews, allows for the knowledge collected in the Define Phase to be expanded upon and rules/logic to be developed under the Analyze Phase. These rules are implemented within the Design Phase and the system is validated through the Verify Phase. The rules and subsequent implementation are utilized in both the data loading (Chapter 5) and the DRPS (Chapter 6). The next Chapter will address two problems that the framework must address to properly function:

1. Comprehensively identify safety information required for each asset (Problem Statement No. 1).

2. Transfer the data from the design and construction phase to the FM phase. 


\section{DATA LOADING AND DATA TRANSFER}

In order to utilize a system that delivers safety information to facility management staff efficiently, an understanding of where and how data is populated and stored throughout the lifecycle of a facility is of paramount importance. During the design and construction phase of a project, relevant safety information applicable to the safe maintenance of a facility is created. This information can be anything from basic location-based information to complex safety information. Regardless of the type of information, in order to deliver it comprehensively to the end user at the completion of a project, the requirements for what data is input and how the data is transferred needs to be addressed within the prime contract(s).

This chapter will address two phases of the overall proposed BIM-based safety framework as presented in Figure 24.

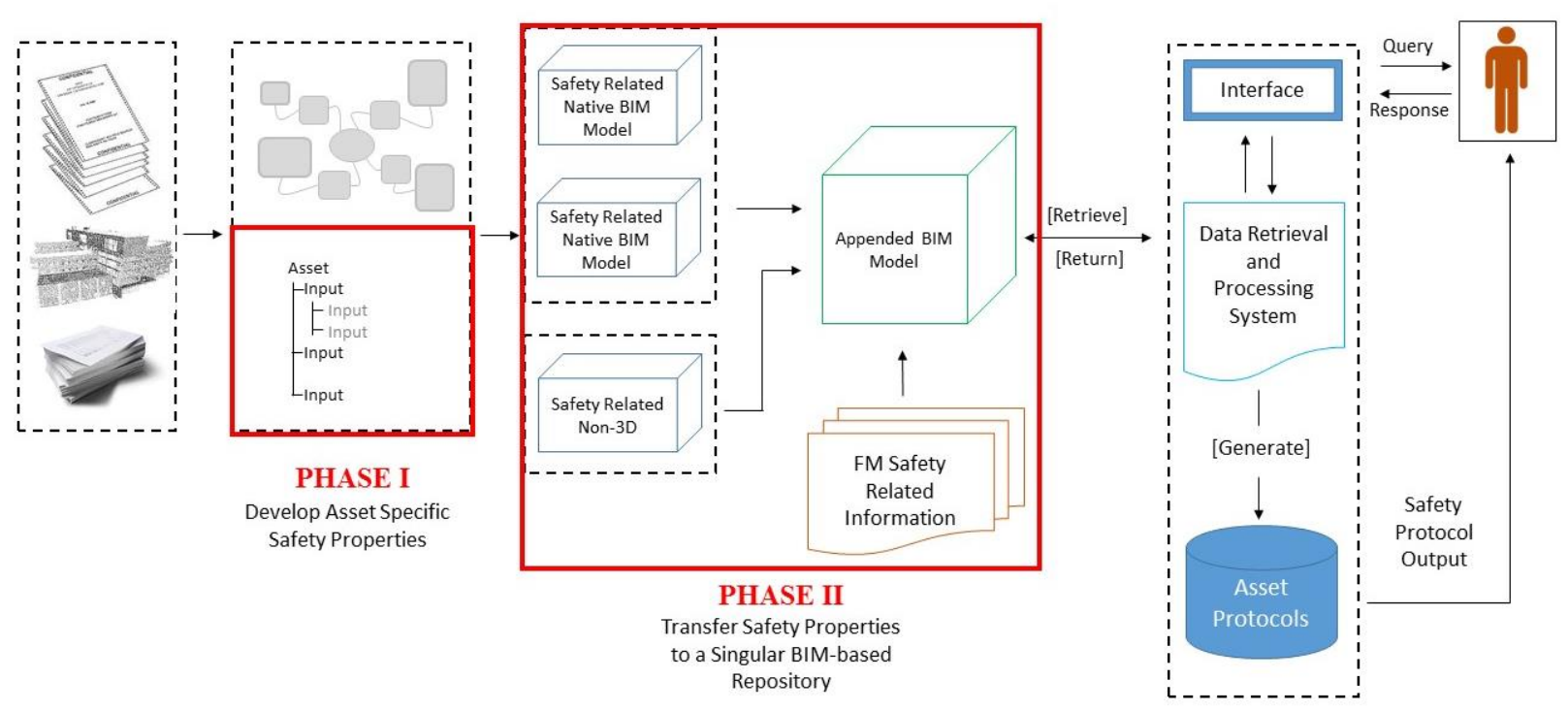

Figure 24: Phase I and Phase II of the BIM-Based Safety Framework

Phase I on Figure 24 presents the Asset Safety Identification Tool (ASIT), a research tool developed in order to verify that the applicable safety inputs are assigned to each piece of equipment based on that equipment's asset group. This tool is utilized in order to create standard safety properties for each asset type relative to facilities management. As noted in Problem Statement No. 1 of this research, "applicable project related safety information is often available at the handover stage from construction to facility management, but is not appropriately identified for facility management tasks." By standardizing the structure of relevant safety information for facility specific assets, a positive step is made towards mitigating safety concerns due to unidentified hazards for a given asset. 
Phase II addresses data transfer - the mechanism that this research utilizes in order to transfer the relevant safety data from its data source to an appended Navisworks model. Although the safety data at this phase has already been identified and structured, the entities creating the information may utilize a number of different mechanisms to transfer the data. Phase II presents a dataflow mechanism utilizing existing software in order to store the data from multiple platforms to a single appended Navisworks model. This model acts as a safety data repository and a platform in which the DRPS can be integrated. The DRPS (Phase III) is presented in Chapter 6 Data Retrieval and Processing System (DRPS).

\subsection{Phase I - Asset Safety Identification Tool (ASIT)}

Chapter 4 of this research identified safety inputs that require a value assigned by the appropriate contract entity (data sourcing) prior to the utilization of the proposed DRPS. The Asset Safety Identification Tool (ASIT) presented in this section serves two purposes in support of the framework:

1. Develops standard, comprehensive safety information for specific FM asset groups.

2. Mitigates the unidentified and unstructured nature of safety information for specific assets when it is submitted to the FM Staff after the design and construction phase.

The ASIT is a tool that when executed can produce safety properties for an asset group by running the asset information through the automated spreadsheet. The ASIT guides the user through safety property development by requiring responses to queries based on the safety inputs identified in Table 20: Safety Inputs and Protocols. Evaluation of the safety inputs revealed a meronomy hierarchy (part-whole relationship) between the inputs. The ASIT captures and processes the meronomy hierarchy using conditional constructs in the form of "if-then" statements. Using information regarding an asset group, a standardized set of safety properties can be developed.

For example, consider three safety inputs identified in Table 20.

- Hazardous Energy / Live Current

- Disconnect Location

- Voltage

In order to perform maintenance on any asset, a FM worker would want to know if the asset requiring maintenance had hazardous energy, a disconnect location, and a voltage. However, evaluation of the inputs reveals the meronomy hierarchy. If the asset does not have hazardous energy / live current, 
then there won't be a disconnect location or voltage to consider. Therefore, a formula can be developed to capture the relational data between the inputs.

IF ("Hazardous Energy / Live Current" = TRUE), THEN "Disconnect Location"

AND "Voltage" > ""

IF ("Hazardous Energy / Live Current" = FALSE), THEN ""

The above states that if the Hazardous Energy / Live Current is "True", then a value must be assigned to Disconnect Location and Voltage. If Hazardous Energy / Live Current is "False", no additional information is required.

The safety inputs within the ASIT are structured within three categories, primary, dependent, and independent. In the above example, Hazardous Energy / Live Current is a primary input. A "yes" response to a primary input, prompts the ASIT to require additional values through dependent inputs. The dependent inputs in the above example are Disconnect Location and Voltage. An independent input is similar to a primary input, requiring a "yes" or "no" response, but does not require additional information and therefore does not carry any dependent inputs. An example of an independent input from Table 20 is Outdoor Environment. A "yes" response to Outdoor Environment notifies the FM user that the asset requiring maintenance is outdoors, but safety protocols associated with the outdoor environment (heat mitigation, cold mitigation, weather considerations, etc.) are variable and therefore are not be stored within the asset.

Using the ASIT, a FM department can develop a comprehensive list of safety properties based on the assets within their facility. These safety properties can then be stored within the singular BIM-based repository and values can be assigned for each asset (Section 5.2 Data Transfer). Through continuous iteration and the use of diverse test cases, a standardized list of safety properties can be developed for all asset groups.

Appendix 10.13 presents the complete list of the interrelated safety inputs as they are formulated in the ASIT. Figure 25 graphically identifies the various parts of the ASIT with a text description of each part on the following page. 


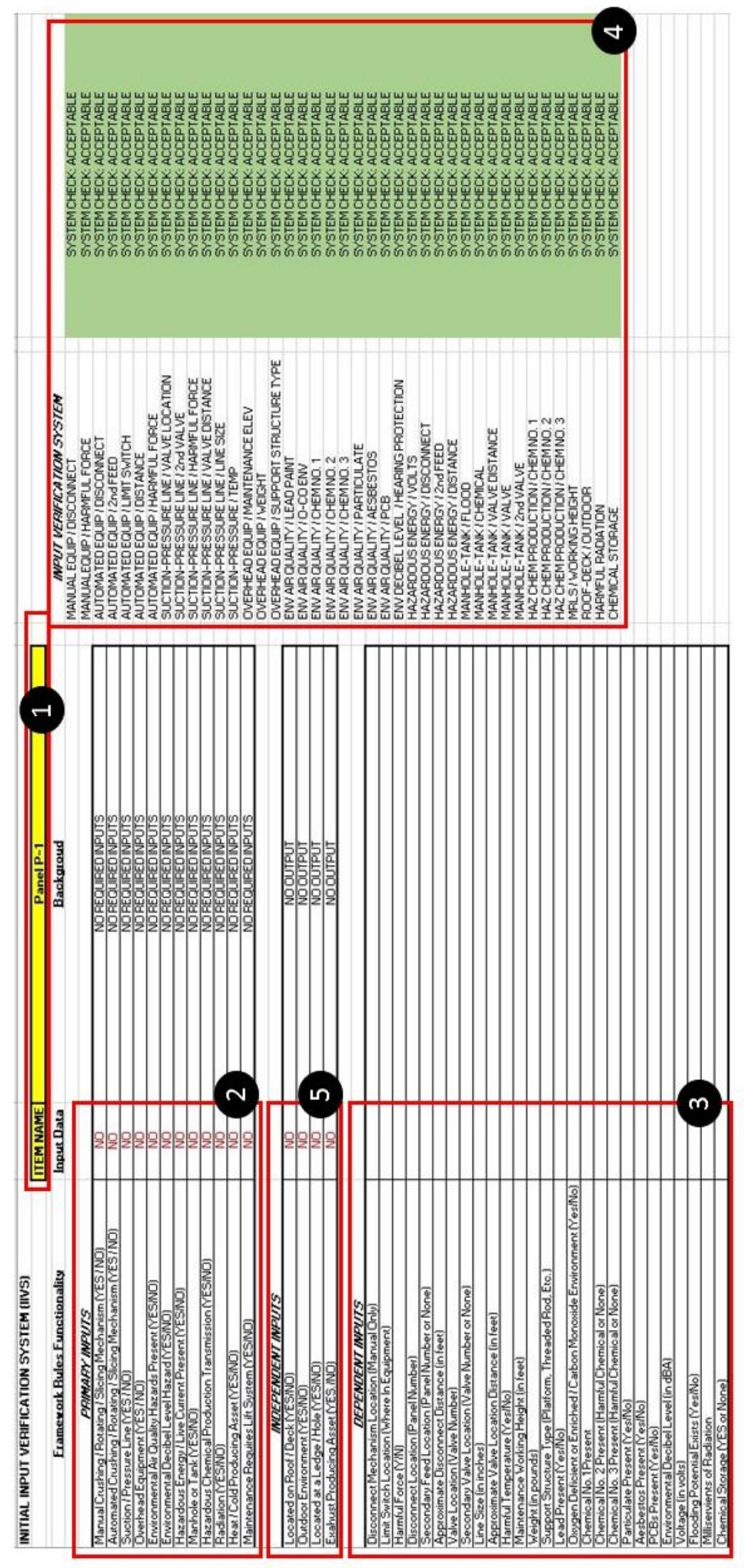

בิ 
1. Item Name - The name of the piece of equipment as it is presented in the model. The name in this box must match the name in the model in order to enact the data transfer presented later in this chapter. Each piece of equipment requiring data loading will have its own worksheet within the spreadsheet.

2. Primary Inputs - A user will respond "Yes" or "No" to the 11 Primary Inputs. A "Yes" response in this section will prompt the user to input additional Dependent Inputs.

3. Dependent Inputs - Based on a "Yes" response to a Primary Input, asset specific information is needed in order to deliver detailed asset information to the FM user. The type of value is dictated by the Dependent Input. For example, the Dependent Input "Harmful Force" requires either a "Yes" or a "No" value, while the "Weight" input requires an input in pounds. Figure 26 presents an example of an active Dependent Input section. In this example, the Primary Input "Hazardous Energy" is given a "Yes" response as Panel P-1 maintains an electrical current. Due to this "Yes" response, the user must populate the values for the associated "Dependent Inputs."
a. Disconnect Location (Panel Number)
b. Secondary Feed Location (Panel Number or None)
c. Approximate Disconnect Distance (in feet)
d. Voltage (in volts)

In order to aid the developer during the safety property identification phase, conditional formatting is used when a safety input requires a value. An example of this is presented in Figure 26 with the inputs requiring a value being highlighted green.

4. Input Verification System (IVS) - In addition to the conditional formatting of the Dependent Inputs, the IVS runs a system check to verify that all of the inputs requiring values have been executed. When presenting a red color, a value is missing for the applicable input. In addition, the IVS will notify the user of what error is occurring within the input system. Upon populating applicable values, the IVS will change back to green and read "System Check: Acceptable." This error checking is depicted in Figure 26.

5. Independent Inputs - Require a "Yes" or "No" response, however, a "Yes" response does not require additional information (i.e. these inputs do not have corresponding Dependent Inputs). These inputs play a role in the DRPS, but during safety property identification only require the binary response.

Figure 27 schematically presents the workflow of the ASIT system. 


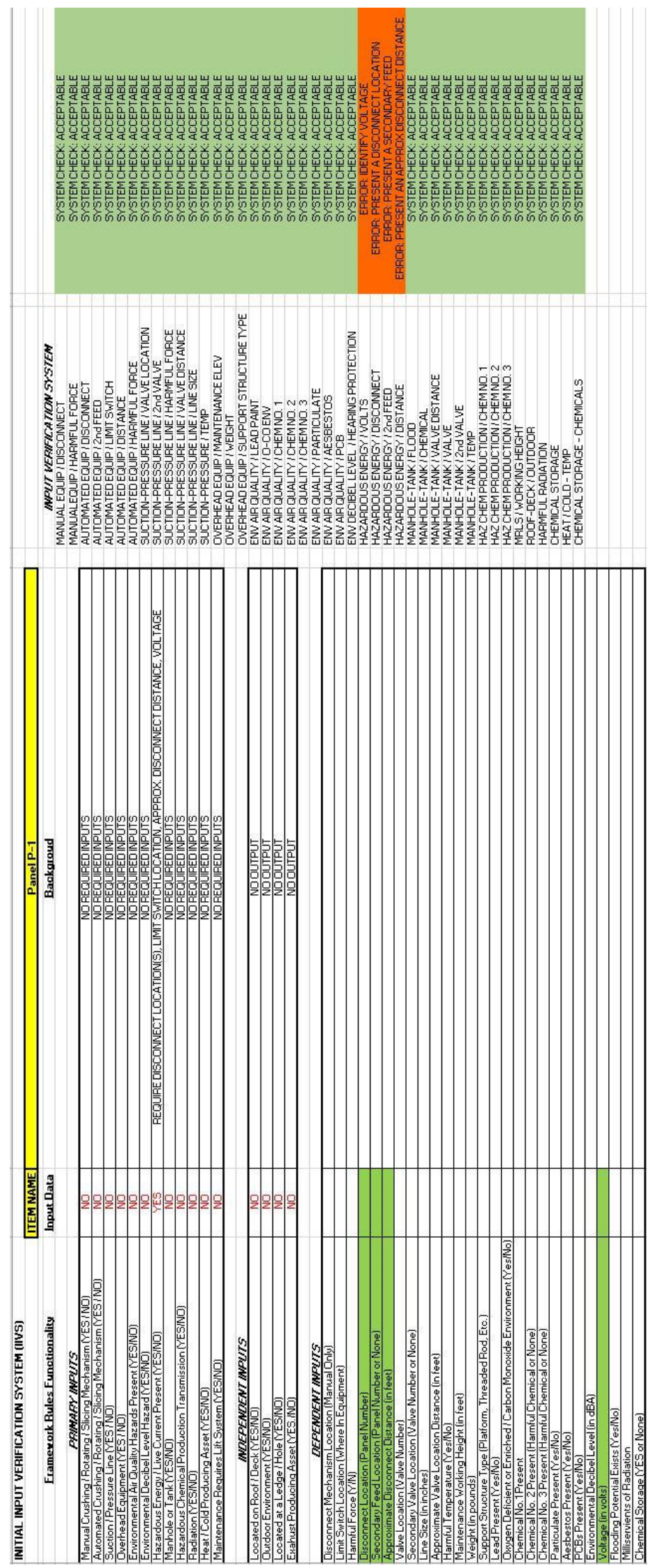

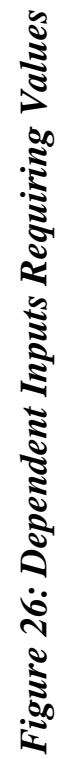



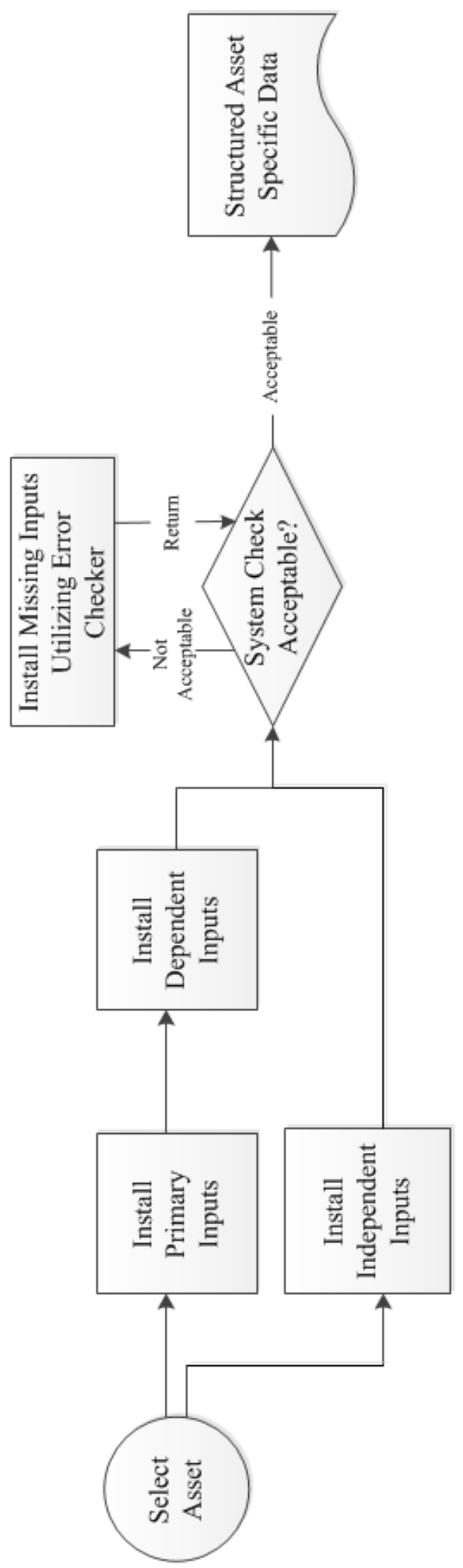

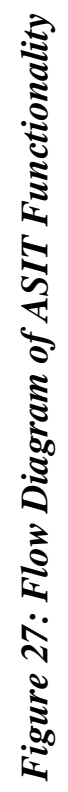

әseपd InduI 
In order to test the ASIT functionality, four diverse assets are run through the system in order to develop standard safety properties for ducts, industrial coal-fired boilers, paper rewinders, and ammonia tanks on an ice rick compressor package. Using known O\&M information regarding the asset type and the ASIT, the following Tables 21-24 present the standardized safety properties for each of the four assets tested.

Table 21: ASIT Developed Safety Properties for Ducts

\begin{tabular}{|c|c|c|}
\hline Input Type & Inputs & Value \\
\hline Primary & Overhead Equipment & $<$ User Input Required $>$ \\
\hline Dependent & Maintenance Working Height & <User Input Required > \\
\hline Dependent & Weight & <User Input Required $>$ \\
\hline Dependent & Support Structure Type & <User Input Required $>$ \\
\hline Primary & Maintenance Requires Lift System & $<$ User Input Required $>$ \\
\hline Dependent & Maintenance Working Height & $<$ User Input Required $>$ \\
\hline Primary & Environmental Decibel Level & $<$ User Input Required $>$ \\
\hline Dependent & Environmental Decibel Level (in dBA) & $<$ User Input Required $>$ \\
\hline Primary & Environmental Air Quality & <User Input Required $>$ \\
\hline Dependent & Asbestos Present & <User Input Required > \\
\hline Dependent & Particulate Present & $<$ User Input Required $>$ \\
\hline Independent & Outdoor Environment & $<$ User Input Required $>$ \\
\hline
\end{tabular}

Table 22: ASIT Developed Safety Properties for IndustrialCFBoiler

\begin{tabular}{|c|c|c|}
\hline Input Type & Inputs & Value \\
\hline Primary & Environmental Air Quality & <User Input Required> \\
\hline Dependent & Oxygen Deficient/Enriched/CO Env. & <User Input Required > \\
\hline Dependent & Particulate Present & <User Input Required $>$ \\
\hline Primary & Environmental Decibel Level & <User Input Required> \\
\hline Dependent & Environmental Decibel Level (in dBA) & <User Input Required > \\
\hline Primary & Hazardous Energy/Live Current Present & Yes \\
\hline Dependent & Disconnect Location & <User Input Required > \\
\hline Dependent & Secondary Feed Location & <User Input Required $>$ \\
\hline Dependent & Approximate Disconnect Distance & <User Input Required $>$ \\
\hline Dependent & Voltage & $<$ User Input Required $>$ \\
\hline
\end{tabular}




\begin{tabular}{|c|c|c|}
\hline Primary & Manhole / Tank / Confined Space & Yes \\
\hline Dependent & Valve Location & <User Input Required> \\
\hline Dependent & Secondary Valve Location & <User Input Required> \\
\hline Dependent & Approximate Valve Distance & <User Input Required> \\
\hline Dependent & Harmful Temperature & <User Input Required> \\
\hline Primary & Heat/Cold Producing Asset & Yes \\
\hline Dependent & Harmful Temperature & Yes \\
\hline
\end{tabular}

Table 23: ASIT Developed Safety Properties for PaperRewinder

\begin{tabular}{|c|c|c|}
\hline Input Type & Inputs & Value \\
\hline Primary & $\begin{array}{l}\text { Automated Crushing/Rotating/Slicing } \\
\text { Mechanism }\end{array}$ & Yes \\
\hline Dependent & Limit Switch Location & <User Input Required $>$ \\
\hline Dependent & Harmful Force & $<$ User Input Required $>$ \\
\hline Dependent & Disconnect Location & $<$ User Input Required $>$ \\
\hline Dependent & Secondary Feed & <User Input Required $>$ \\
\hline Dependent & Approximate Disconnect Distance & $<$ User Input Required $>$ \\
\hline Primary & Hazardous Energy & Yes \\
\hline Dependent & Voltage & $<$ User Input Required $>$ \\
\hline Dependent & Disconnect Location & $<$ User Input Required $>$ \\
\hline Dependent & Secondary Feed & $<$ User Input Required $>$ \\
\hline Dependent & Approximate Disconnect Distance & <User Input Required $>$ \\
\hline Primary & Maintenance Requires Lift System & <User Input Required > \\
\hline Dependent & Working Height & $<$ User Input Required $>$ \\
\hline
\end{tabular}

Table 24: ASIT Developed Safety Properties for AmmoniaTank

\begin{tabular}{|c|l|c|}
\hline Input Type & Inputs & Value \\
\hline Primary & Suction/Pressure Lines & Yes \\
\hline Dependent & Valve Location & <User Input Required> \\
\hline Dependent & Line Size & $<$ User Input Required> \\
\hline Dependent & Harmful Force & <User Input Required> \\
\hline Dependent & Secondary Valve Location & <User Input Required> \\
\hline Dependent & Approximate Valve Location Distance & <User Input Required> \\
\hline
\end{tabular}




\begin{tabular}{|c|c|c|}
\hline Primary & Environmental Air Quality & Yes \\
\hline Dependent & Chemical No. 1 Present & Liquid Anhydrous Ammonia \\
\hline Dependent & Chemical No. 2 Present & $<$ User Input Required> \\
\hline Primary & Environmental Decibel Level Hazard & $\langle$ User Input Required $>$ \\
\hline Dependent & Environmental Decibel Level & $<$ User Input Required $>$ \\
\hline Primary & Manhole / Tank / Confined Space & Yes \\
\hline Dependent & Valve Location & $<$ User Input Required $>$ \\
\hline Dependent & Secondary Valve Location & $<$ User Input Required $>$ \\
\hline Dependent & Harmful Temperature & <User Input Required $>$ \\
\hline Dependent & Approximate Valve Location Distance & $<$ User Input Required $>$ \\
\hline Dependent & Chemical Storage & $<$ User Input Required $>$ \\
\hline Primary & $\begin{array}{l}\text { Hazardous Chemical } \\
\text { Production/Transmission }\end{array}$ & Yes \\
\hline Dependent & Chemical No. 1 Present & Liquid Anhydrous Ammonia \\
\hline Primary & Heat / Cold Producing Asset & Yes \\
\hline Dependent & Harmful Temperature & <User Input Required > \\
\hline Primary & Overhead Equipment & <User Input Required > \\
\hline Dependent & Maintenance Working Height & $<$ User Input Required $>$ \\
\hline Dependent & Weight & <User Input Required $>$ \\
\hline Dependent & Support Structure Type & $<$ User Input Required $>$ \\
\hline Primary & Maintenance Requires Lift System & <User Input Required $>$ \\
\hline Dependent & Maintenance Working Height & $<$ User Input Required> \\
\hline Independent & Outdoor Environment & $<$ User Input Required $>$ \\
\hline
\end{tabular}

In Chapter 7 System Architecture Validation, the developed standardized safety properties are validated based on specific FM test cases. Utilizing the particulars presented in each test case (i.e. environment, asset location, actor, etc.), values are assigned to the asset and comprehensive safety information is available for the DRPS to process.

As more assets utilize the ASIT for safety property identification, an expanding list of assets are available to design, construction, and FM users for data loading.

For each asset group in any project, the standardized safety properties must be stored by the design/construction professionals within one of two repositories. The properties can be input into a native 
model (e.g. the mechanical model developed by the mechanical subcontractor), or the information can remain within the ASIT spreadsheet. As an Excel spreadsheet the properties and values stored in the ASIT can be easily copied over in order to execute a data transfer as presented in Section 5.6 Data Transfer Mechanism for BIM-Based Framework of this chapter. Regardless of the method of storage, transferring the safety data from the design and construction phase to the FM phase is challenge that requires resolution in order for the BIM-based Framework to function properly. The next section will cover Phase II - Data Transfer, presenting the challenges of data transfer, the current market solutions, and the transfer mechanism this research utilizes.

\subsection{Phase II - Data Transfer}

Upon completion of Phase I, applicable safety data needs to be transferred into an Appended BIM Model as presented in Figure 24. In an effort to continuously streamline the process of data transfer, a significant amount of research and development has been placed on the utilization of building information models as O\&M data storage repositories. Utilizing BIM to store O\&M data throughout the lifecycle of the project allows for an Owner to obtain a comprehensive model at the completion of the project. However, this process of BIM data transference is still in its infancy and although great strides have been made, many handover issues still exist (Cleveland 2014). This section will explore the use of building information models as a data transference tool for handover of applicable data to be used during the facilities management phase.

\subsubsection{Data Handover Challenges}

The transfer of data from the design and construction phases to the facility management phase has remained greatly unchanged for years. Hard copy/electronic O\&M manuals, file sharing, and project management hubs, is currently the industry standard. The handover process of information necessary for facility management (e.g. as-built drawings, operations and maintenance manuals, parts list, etc.) continues to deliver such information using a combination of paper printed documents and digital versions of printed documents. This combination of paper-based and digital-based formats have proved unsuitable for the use, maintenance, and management of such information. The majority of the information delivered is still held in documents that do not have a formal structure, making it difficult and time consuming for direct input and interpretation by the computerized facility management system. Those who need to utilize the information provided must go through the additional step of reading the documents, synthesizing and extracting the needed information, and transforming the data into a structured format before inputting the information into the FM system. 
Moreover, design and construction documents are not typically turned over to the FM staff until the end of the construction phase. Waiting until the completion of the construction phase / project handover to deliver the needed information, in a largely unstructured format, results in several problems. Mainly, requiring FM staff to recreate and assemble information that has been developed by others, resulting in a slowed handover process, a reduction in the ability to find all the necessary information, and an increased chance of errors and omissions through the time-constrained transcription process.

In addition, issues associated with the current approach of information transfer is the suitability of the information format for later use during operations and maintenance. Fallon and Palmer (2006) defined four major categories of information forms and formats as shown in Figure 28. Information is either structured or unstructured. Both can be proprietary or standard.

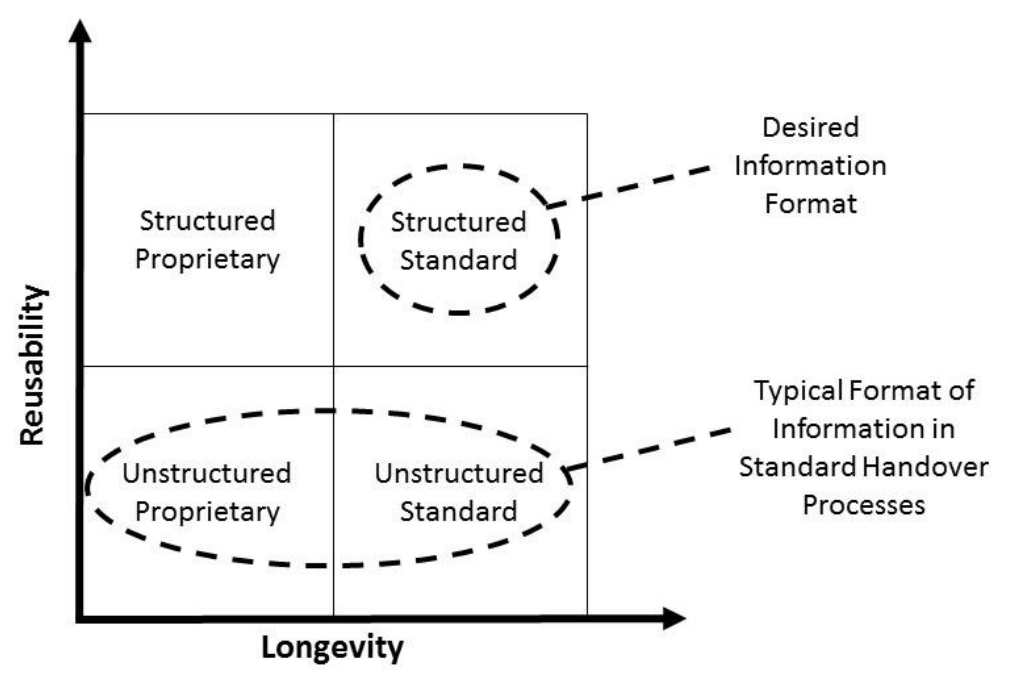

Figure 28: Longevity and Reusability of Information Forms and Formats (adopted from Fallon and Palmer, 2006)

"Structured data" can be accessed and manipulated directly by computer programs without human intervention and is the preferred form to be used in downstream automated processes or when regular updates are required. This form allows for automated — and therefore cost effective — search, retrieval, and update, while maintaining the intelligent information content. Structured information may be quantitative, descriptive, or graphical. There are a number of proprietary structured data models. Examples of structured data formats include information fields defined by the Construction Operation Building Information Exchange (COBie) (East 2007).

"Unstructured Data" is any data that cannot be machine-interpreted, requiring manually intensive interpretation and transcription by the facility staff. Examples include electronic images and electronic .pdf 
documents. This form of information is suitable for read only access but unsuitable for an automated framework.

Proprietary formats, also referred to as "native" formats, are created by and are the property of specific software vendors such as CAD programs. Longevity of such formats is sensitive to changes and modifications made by the vendor or discontinuation of the product, rendering the proprietary data unusable.

Standard formats include "Ad hoc" standards or "Formal" standards. "Ad hoc" standards refer to proprietary formats that have been made publically available and are supported by multiple vendors with published specifications. This assures data longevity. Examples include DXF and PDF. "Formal" standards are supported and maintained by official standards organizations such as the Industry Foundation Classed (IFC) maintained by the Building Smart Alliance (National Institute of Building Sciences buildingSMART alliance 2014). In addition to longevity of data stored in this format, it is also more flexible and useful given the format is typically developed through a consensus process.

The majority of the information submitted at handover is unstructured, with some being proprietary, and others standard. This is represented by the two lower left and right quadrants in Figure 29. The most efficient format for the purpose of supporting maintenance and operations is structured and standard information. As shown in the top right quadrant of Figure 29. Utilizing a standard and structured format greatly mitigates the need to identify, synthesize, and transform the data.

Within the construction industry and amongst facility owners, there is an interest in the utilization of BIM during the FM phase (Becerick-Gerber et al. 2012). The use of BIM as a repository of information is an intuitive step in the evolution of the submittal and O\&M process, however the usage of BIM for data transference continues to be rare. The minimal use of BIM as a data transference tool can be attributed to a number of issues. Issues such as model updates, a shortage of BIM skills by FM staff, a lack of collaboration between project and end user stakeholders, contract and legal framework, and interoperability, all contribute to the low utilization of BIM for FM (Becerick-Gerber et al. 2012; East and Brodt 2007; Kelly et al. 2013; Teicholz 2013).

Arguably, the most complex issue faced by project teams, and where a significant amount of research has taken place, is interoperability. Within any project, a number of software may be utilized to design the facility, plan the work, store and exchange the information, and execute FM tasks. This creates a mismatch 
of file extensions and proprietary systems that cannot communicate together (Cleveland 2014). Recently, a significant push has been made to incorporate operations and maintenance information into BIM models for facilities maintenance and the linking to FM software. Through the use of BIM interoperability, virtual databases, and add-ons such as COBie, an improvement in O\&M storage and retrieval has been achieved to some extent; however, these systems are still not evolved enough to handle all the interoperability. Due to the remaining inefficiencies within the system interoperability, a number of current market solutions have been developed to work-around the issues.

\subsubsection{BIM-FM Data Transfer Current Market Solutions}

The industry is quite aware of the handover issues and a great deal of research and development has been done in order to mitigate the problems. To date, five broad strategies for data handover exist in order to circumvent current market deficiencies - hard entry, interoperability, middleware, Open Database Connectivity (ODBC), and Application Program Interface (API). The decision to utilize one or more of these strategies, independently or in tandem, is based on a number of factors including existing hardware and software infrastructure, worker training, budget, and user system goals. A greater explanation of each strategy are as follows:

1. Hard Entry - Utilizes attribute and value data inputs. Inputs can be stored in a 3D model or nonmodel format (i.e. Microsoft Excel).

2. Interoperability - Utilizes software compatibility to transfer relevant information from the native/design file to a different software.

3. Middleware - A compatibility "bridge" that allows for non-interoperable software applications to transfer information (i.e. EcoDomus).

4. Open Database Connectivity (ODBC) - An open standard application for accessing an existing database.

5. Application Program Interface (API) - Sends a programming request to a data source with instructions on how to develop a response, and returns the response.

Often, in order to effectively execute data handover, two or more strategies may be utilized in tandem. Although handover issues are complex and current market systems remain imperfect, many problems can be circumvented with proper planning. Utilizing data exchange frameworks, analysis, and modeling, researchers and developers are actively working to synthesize and seamlessly bridge the gap between design, construction, and FM. 
This section reviews three real life examples that utilized data transference and data storage in order to utilize BIM for FM. All of the examples below were executed in order to transfer O\&M information in lieu of the safety information that is transferred within the proposed safety framework. Although the variation on data type exists, the processes/procedures employed are still relevant. This section will not present all the possible methods or software combinations and is not intended to present a comprehensive list of all BIM-FM examples, rather it is intended to show that data transference is possible if the appropriate planning takes place. It should be noted that although these instances would be considered successful data transference examples, each case identified limitations and issues associated with the transfer.

The examples presented here utilize summaries and schematic graphics to present data transference. Schematic graphics are intended to present a general flow of data between phases and software. The following examples all utilize Autodesk Revit in some capacity as a design software and/or O\&M information repository. This circumstance is a reflection of the software popularity, not an inclusion criteria of this section.

\subsubsection{Case 1: Using Hard Data Entry, Interoperability, \& Middleware [EcoDomus] for BIM-FM Data Exchange}

The USC School of Cinematic Arts initiated a project in 2007 requiring the construction of six buildings in three phases (Teicholz 2013). BIM was utilized for architectural, structural, and MEP disciplines and the implementation of BIM-FM was required from the start of the project. The USC case study is unique in the fact that as the phases progressed, so too did the requirements for BIM-FM implementation. This case presents a project team that evolved along with the technologies and transference limitations. Figure 29 graphically presents the data transference path for the USC Case Study. 


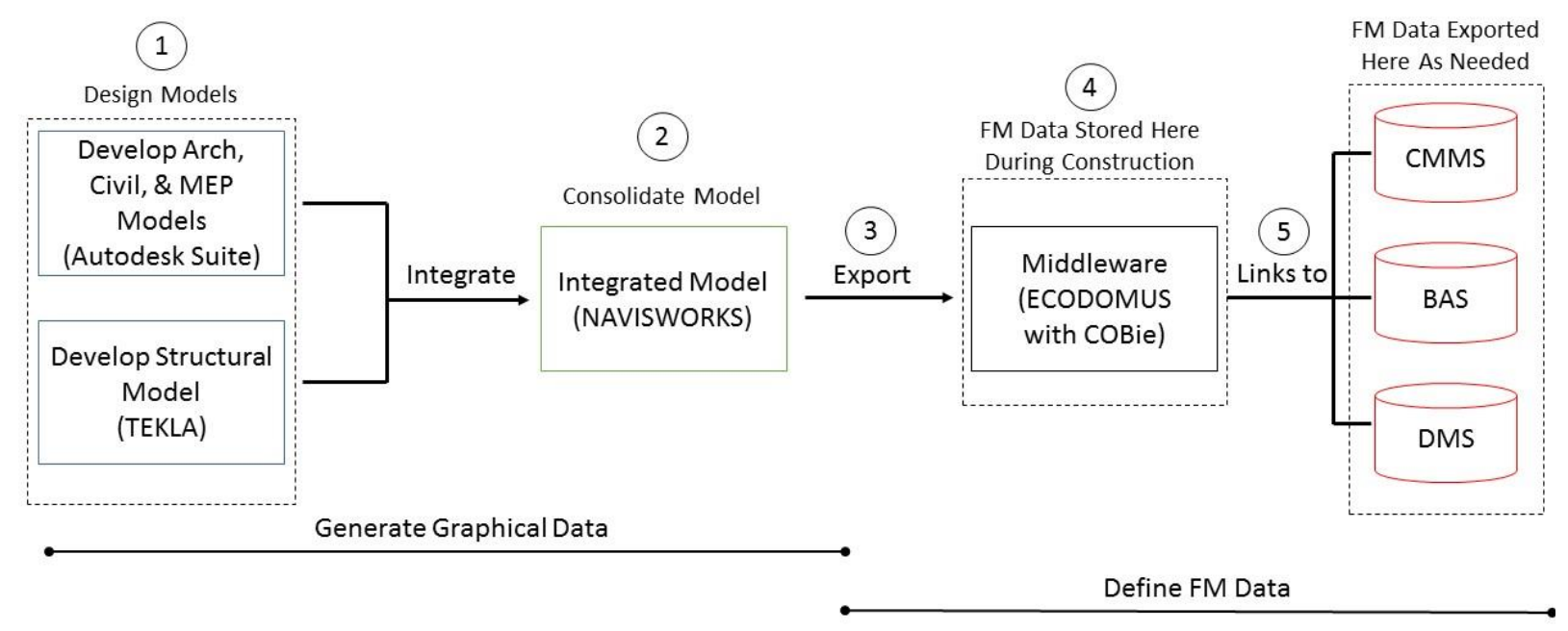

Figure 29: Data Transference Path for USC Case Study

The following presents the flow of data utilizing the numbers presented within Figure 29.

1. Architecture, MEP, Civil, and some structural models were created in the Autodesk suite, while the structural engineer utilized Tekla for structural design.

2. The various models were appended into Navisworks to create a comprehensive model and utilize tools such as clash detective for trade coordination. The data being transferred at this point is largely graphical.

3. The comprehensive model along with some FM applicable data was exported to EcoDomus, a middleware software (EcoDomus Inc. 2016).

4. EcoDomus was the primary repository for FM applicable information, as well as the mechanism for transferring data to USC's three online based FM software (CMMS, BAS, and DMS). Much of the data placed into EcoDomus was hard entered into the system or transferred from models via COBie.

5. EcoDomus software also maintained direct links to the online based FM software for easy reference.

As previously mentioned, the project team was aware of the requirements for implementing BIM-FM, but a detailed understanding of what that entailed continued to evolve along with the project. Aside from these planning issues, a few software limitations were identified within this case study. These limitations are presented below. 
- At the time of this case study, direct transference could not be executed between the BIM authoring tools (Revit \& Navisworks) to the CMMS System. This required the use of EcoDomus, as a true middleware, for items created in Revit that are better suited to be stored in the CMMS System (i.e. component schedules).

- Not all subs maintained a Revit license and therefore the use of 3D models during the construction phase needed to be exported as 3D DWGs and imported into compatible software.

- The use of COBie wasn't implemented until Phase 3 (the final phase) and therefore a definition of what data was needed to be transferred and how that data is stored was vague and disorganized leading up to that point.

This case study presents a number of complex software and planning issues. In spite of that fact, it stands as an excellent example of problem mitigation and team collaboration. This case successfully transferred and organized significant amounts of data utilizing software with minimal interoperability. The use of middleware (EcoDomus) played a large role in this implementation, as a data storage and transference mechanism. As interoperability continues to evolve between FM software and design/coordination software, the cost and software requirements for BIM-FM will likely improve.

\subsubsection{Case 2: Using Interoperability \& Middleware [FM Interact] for BIM-FM Data Exchange}

Mathworks, Inc. initiated a project in 2005 to add a building to their existing corporate campus (Teicholz 2013). A major factor in the award of the contract was the implementation of BIM. Similar to many owners implementing BIM-FM for the first time, the level of development and requirements for data evolved along with the project. Additionally, many of the subcontractors had various levels of 3D modeling capabilities and therefore a third party BIM consultant was utilized to create a comprehensive Revit model. Figure 30 presents the data transference path for this project. 


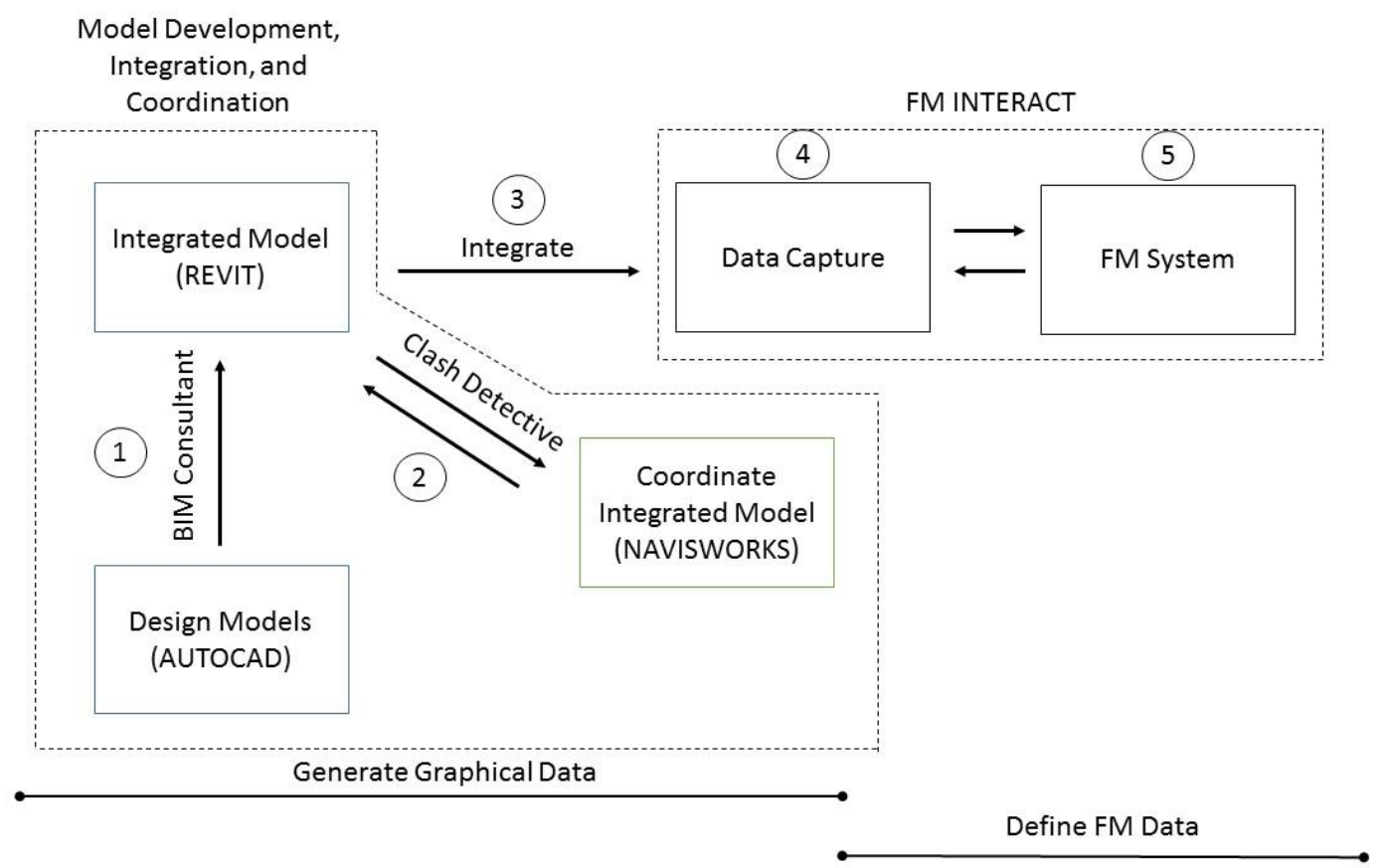

Figure 30: Data Transference Path for MathWorks, Inc. Case Study

The following presents the flow of data utilizing the numbers presented within Figure 30.

1. A BIM Consultant is contracted to develop 2D AutoCAD models, completed by the various project team entities, into a comprehensive 3D model with a link to the existing 3D architecture shell, core, and interiors. Ultimately, a linked Revit model would serve as the final comprehensive model. Data during this phase of the project is almost exclusively graphical.

2. The linked model uses clash detective within Navisworks to prevent conflicts within the 5 linked models.

3. The BIM model, along with the equipment model, properties, and manufacturing information assigned to each piece of equipment, is integrated into FM Interact. These systems have built in interoperability.

4. Once integrated into FM Interact, data can be added or adjusted within the Revit model through the FM Interact add-in.

5. FM staff can access important FM data and model graphics through the FM Interact online portal.

The limitations encountered by the project team are as follows.

- As with many projects involving subcontractors, not all project entities had 3D capabilities. In many cases, implementation of BIM-FM would require the 2D drawings be developed into a 3D 
model. In addition to the need to redesign the subcontractor's drawings into a 3D model, which takes time and money, relevant data needs to be added later as the 3D models become available.

- Initially, the owner was not aware of the information they would need for FM. COBie was later used as a reference source, but not as a data transfer mechanism. Without using COBie as a data transfer mechanism, applicable information had to be hard entered into the system.

This case study is a good example of a project that contains varying levels of 3D modeling capabilities. The implementation of BIM-FM requires that all applicable systems be modeled. Without these capabilities, additional considerations and cost allocations are needed to get these systems into the appropriate format.

\subsubsection{Case 3: Using Interoperability and Proprietary Systems [BIMFMM] for BIM- FM Data Exchange}

The BIMFMM Case study is a research project executed by Lin and Su (2013) that utilizes Revit, Navisworks, Open Database Connectivity (ODBC) and a number of subsystems to create the BIMFMM mobile system. The BIMFMM system was designed to allow for FM staff to access real-time BIM models and relevant FM data within the field utilizing a mobile device. Figure 31 presents the data path of relevant FM information when implementing the BIMFMM system.

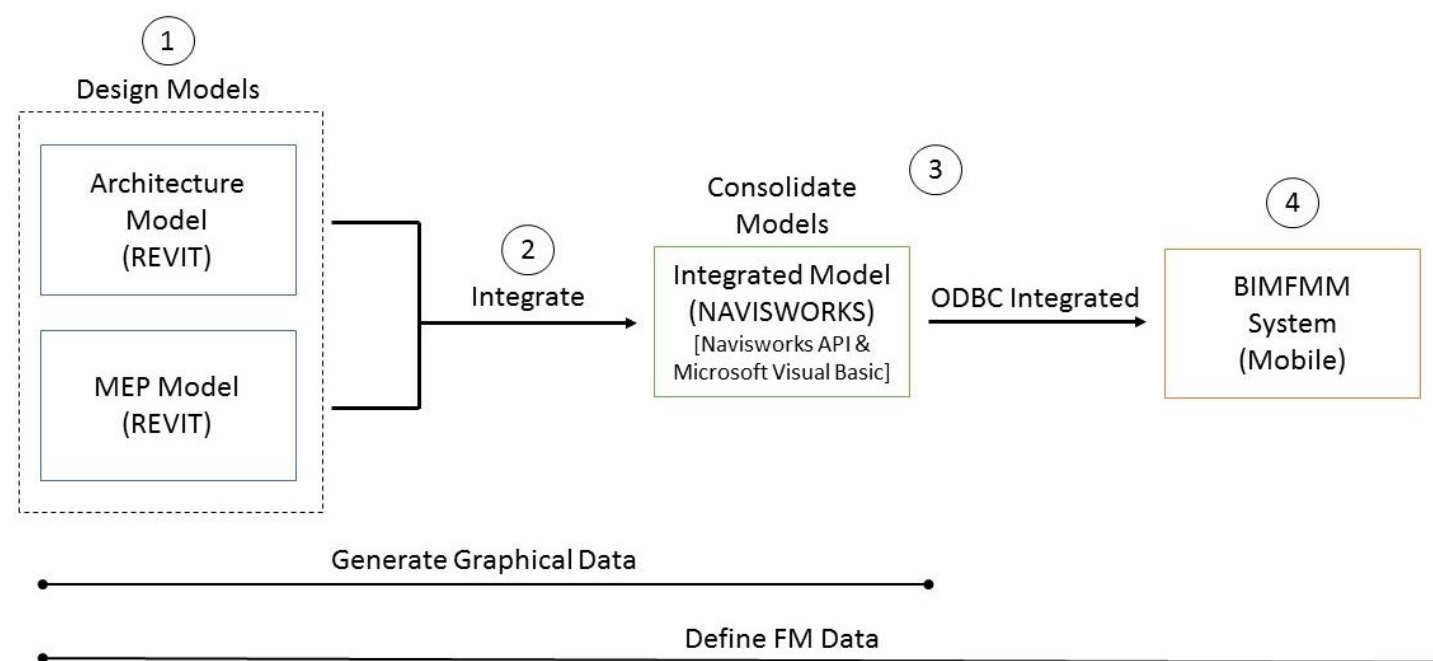

Figure 31: Data Transference Path for BIMFMM Case Study

The following presents the flow of data utilizing the numbers presented within Figure 31 . 
1. Revit Architecture and Revit MEP are utilized for graphical representation of the facility, as well as data storage. The BIM models retained basic description data, parameter-related information, maintenance records, and facility maintenance management reports.

2. Navisworks was utilized as a model integrator to create a comprehensive model.

3. Information integration between the 3D models was achieved by writing code using the Navisworks API and Microsoft Visual Basic.NET programming language. This was done in order to filter the needed information and eliminate the information that was not relevant to the system. Lin and $\mathrm{Su}$ (2013) describe the system integration as follows,

"The BIMFMM system was developed by integrating the $3 D$ BIM models of facilities and maintenance-related information using Navisworks API programming. Open Database Connectivity $(O D B C)$ was utilized to integrate acquired data from different software programs and all maintenance information, such that BIM files can be exported to an ODBC database for connection with the BIMFMM system."

4. The BIMFMM system utilizes a number of internet-based subsystem modules and SQL servers to provide up-to-date information to the mobile devices in the field.

The limitations encountered by the project team are as follows.

- BIM models require constant updates as new equipment is purchased, maintained, or replaced. A system needs to be in place to address these future updates.

- As data is integrated within the BIM models, the NWD files become quite large and cumbersome to download from the SQL servers. Downloads would range from 2-5 minutes.

\subsubsection{Summary}

As the above examples show, there are multiple ways to circumvent the interoperability issues inherent with varying software applications and various levels of modeling capabilities. To date, there is no single accepted method of data transfer between platforms. By continuously testing new and varying transfer processes, valuable "take-aways" can be identified from a given system based on the success or failure of a transfer mechanism. Through the lessons learned, the data transfer mechanisms employed within the proposed safety framework is developed.

\subsubsection{Data Transfer Mechanism for BIM-Based Safety Framework}

For this research, two basic input phases are utilized as shown in Figure 32, a combined design and construction phase and a FM phase. Through multiple avenues of input, from different contract entities, at 
various times in a building's lifecycle, safety relevant information can be stored within the appended Navisworks model.

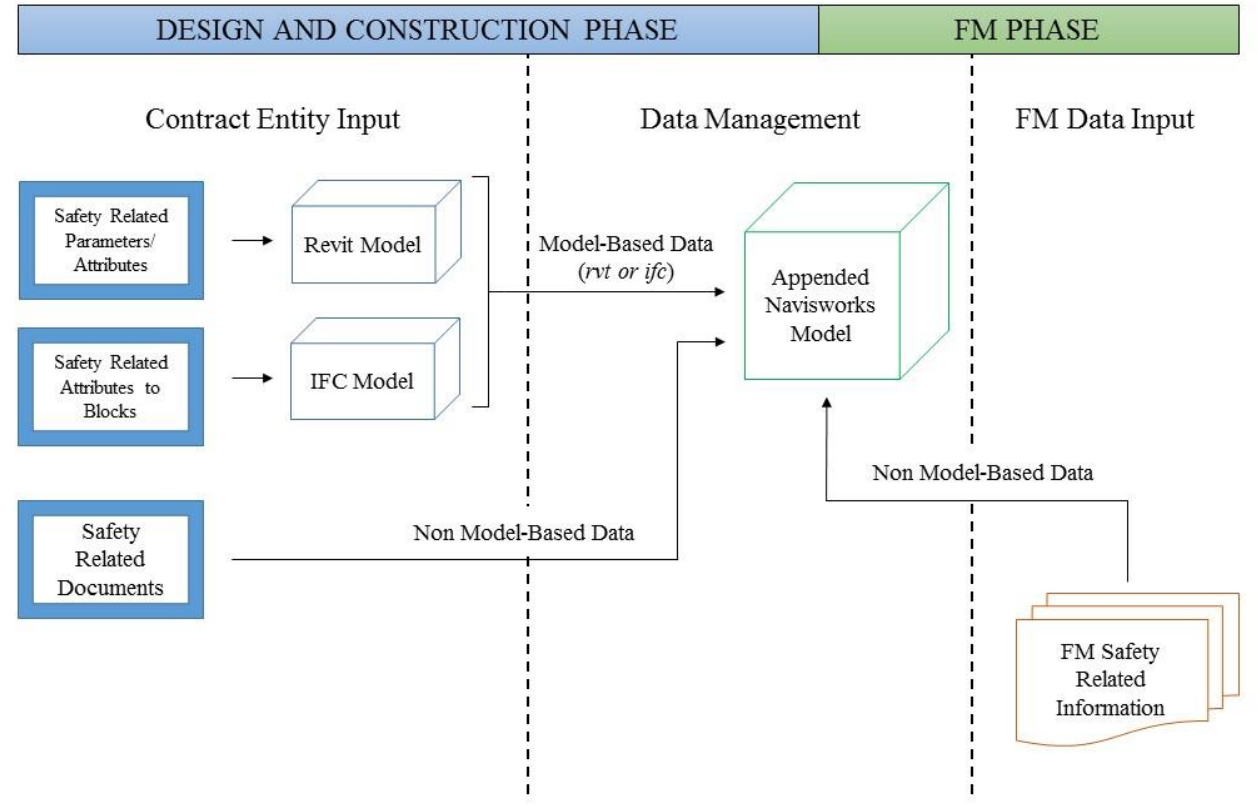

Figure 32: Data Inputs at Three Input Phases

In conjunction with the multi-phased input, this research incorporates two of the four data handover strategies presented in Section 5.2.2 - Interoperability \& Hard Entry.

1. Data Input Through Interoperability:

Relevant safety information captured by the design/build team (i.e. contract entity input) are captured and stored as parameters or attributes in the native models that are directly imported (i.e. appended) in the federated Navisworks model. This model-based approach for data capture and exchange can use various file formats for the 3D model (populated with the necessary safety relevant data) including .rvt or .ifc formats.

2. Hard Entry:

Non model-based relevant safety data produced during the design and construction phases or additional needed safety information defined by FM staff during the O\&M phase are entered into the appended Navisworks model using direct input, utilizing two data entry tools: Selection Inspector and DataTools. 
Figure 32 graphically presents the overall data transfer mechanism employed within the BIM-based safety framework, while Section 5.2.5 provides a proof concept example.

The following is a detailed description of the different steps of Figure 33:

1. Relevant safety information generated by various contract entities (designers, consultants, GC, and trades) are captured and stored as parameters/attributes in corresponding native models and appended into a single federated BIM using Navisworks. This model-based approach of data loading a model is efficient, as interoperability between many file extensions and Navisworks allows for a graphical and data transfer.

When appended in the Navisworks environment, safety data from each model is grouped under a different tab with a system-defined name dependent on the native file format imported.

In order to make changes to the model-based data, add other non model-based data (generated by contract entities or FM staff), or reorganize and group all data under a single tab with a user defined name, data needs to be exported, modified, then imported back to the federated model. This is achieved using the export and import data manipulation tools from Navisworks: Selection Inspector and DataTools respectively. 


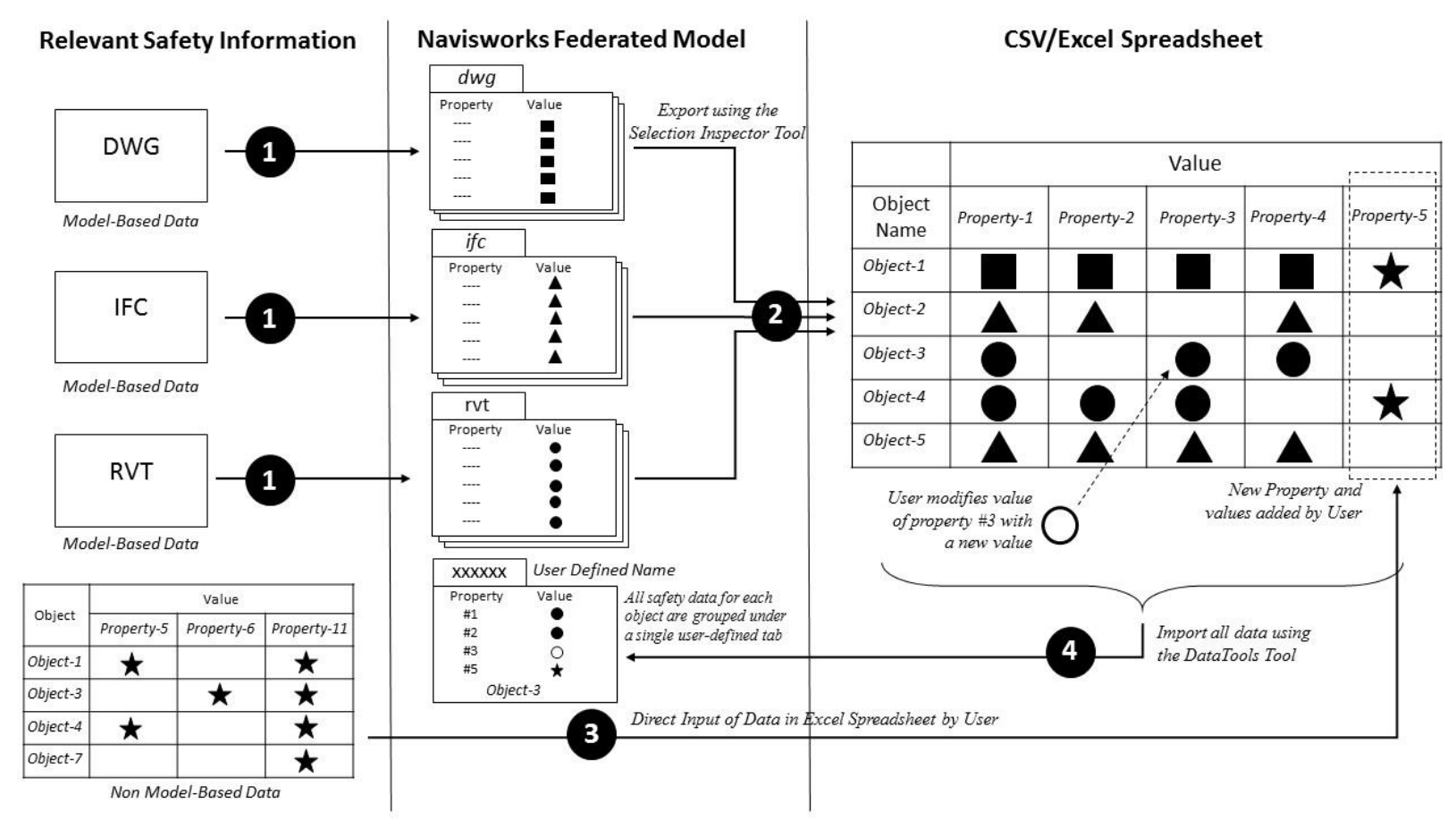

Figure 33: Data Transfer Mechanism within the Safety Framework

2. Using the Selection Inspector tool in Navisworks, appended safety information can be filtered for graphical objects representing the specific equipment targeted for maintenance. Data is the exported to a CSV file and save as an Excel file for modifications. Edits to the data can be manually entered in the Excel spreadsheet

3. Non model-based relevant safety data is directly input by the user in the excel spreadsheet. The user can define new properties (columns) and values for the corresponding objects, or can modify existing properties exported.

4. Once all modifications have been made and new properties and values are added, all data is imported back into the appended Navisworks model using the DataTools function. A user-defined name can be given for the new tab under which all appended safety information will be saved for each graphical object.

a. Additional safety data can be added directly within Navisworks with the Add New User Data Tab function. This allows for some flexibility of data input as long as the amount of information to be added is small.

Once all data modifications and manipulation are complete, all relevant safety information for the equipment targeted for maintenance can be exported and used to interact with the DRPS. 


\subsubsection{Data Transfer Mechanism Proof of Concept}

This section presents an example of the data transfer mechanism described in Figure 32. The proof of concept model, presented in Figure 34, is a representative model of a compressor room in an ice skating rink. This model presents a partial compressor package and electrical system with many of the room and compressor elements removed in order to provide clarity to the equipment being utilized within the proof of concept. By presenting a compressor room, a number of hazards can be presented in a single space. Hazards present within an ice skating compressor room include hazardous energy, chemicals (ammonia), automated rotating mechanisms (motors), hazardous decibel levels, overhead equipment, pressurized lines, and heat producing assets. In addition, Figure 34 presents the equipment that this proof of concept will utilize, a $480 \mathrm{~V}$ electrical panel, a pump, and a compressor.

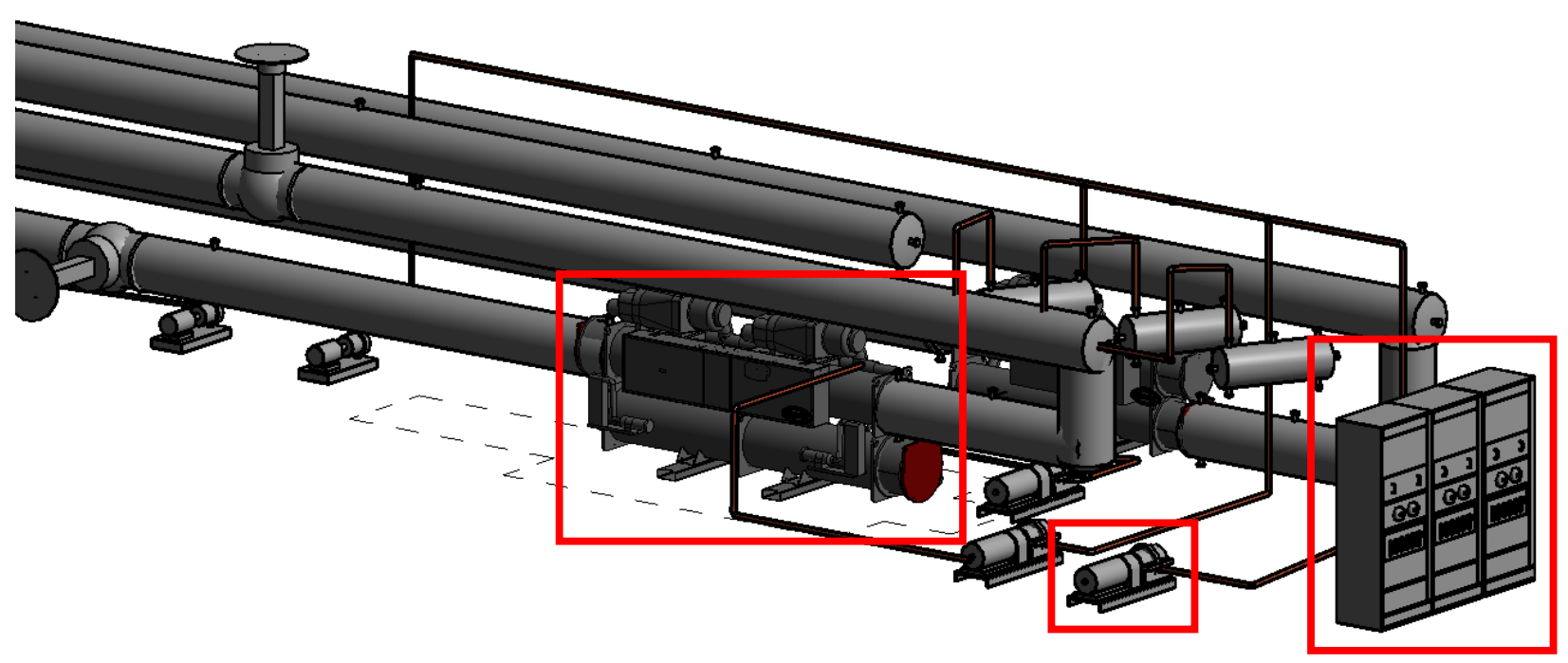

Figure 34: Proof of Concept Model-Compressor Room

In this proof of concept, applicable safety information is loaded into the graphical model through three data transfer mechanisms. First, existing information is input into the native design file developed in Revit 2016. This is executed through the use of shared/project parameters that are assigned to pieces of equipment. This phase simulates the input of data at the design/construction phase, or the "Native BIM-Model" method of transfer presented in Table 20. Once shared or project parameters are added to the project and input into the various applicable equipment, the properties and associated values can be manipulated through Schedules/Quantities in Revit 2016. Completion of the "Native BIM-Model" input phase is succeeded by a model export from Revit 2016 into Navisworks Manage 2016. Bringing the design model into Navisworks allows for other models in compatible formats (.rvt, .ifc, .dwg), executed by others, to be brought into a single model space for coordination. Once appended into Navisworks, the safety inputs within the various models are available in the "Properties Tabs" of each piece of equipment. The name of this tab, the 
presentation of the safety inputs, and the other properties available within each tab is a function of the native file extension being brought into Navisworks.

Table 25 presents the "Native BIM-Model" inputs utilized in this proof of concept and Figure 35 presents how the information is presented in Navisworks Manage 2016.

Table 25: Proof of Concept Data Inputs

\begin{tabular}{|l|l|l|}
\hline Equipment & Property & Value \\
\hline \multirow{5}{*}{ Pump } & Electrical Disconnect Location & Panel 103A \\
\cline { 2 - 3 } & Voltage & $460 \mathrm{~V}$ \\
\cline { 2 - 3 } & Approx. Disconnect Distance (in feet) & 4 \\
\cline { 2 - 3 } & Valve Location & V106 \\
\cline { 2 - 3 } & Approx. Valve Distance (in feet) & 8 \\
\cline { 2 - 3 } & Heat Producing Asset & Caution - Hot \\
\hline \multirow{5}{*}{ Electrical Panel } & Voltage & $480 \mathrm{~V} / 3-0 \mathrm{VA}$ \\
\hline & Electrical Disconnect Location & Local \\
\cline { 2 - 3 } & Approx. Disconnect Distance (in feet) & 0 \\
\hline & Voltage & $480 \mathrm{~V} / 3 \mathrm{ph} / 60 \mathrm{~Hz}$ \\
\cline { 2 - 3 } & Electrical Disconnect Location & Panel 103B \\
\cline { 2 - 3 } & Approx. Disconnect Distance (in feet) & 12 \\
\cline { 2 - 3 } & Automated Rotation & Yes \\
\cline { 2 - 3 } & Heat Producing Asset & Caution - Hot \\
\hline
\end{tabular}

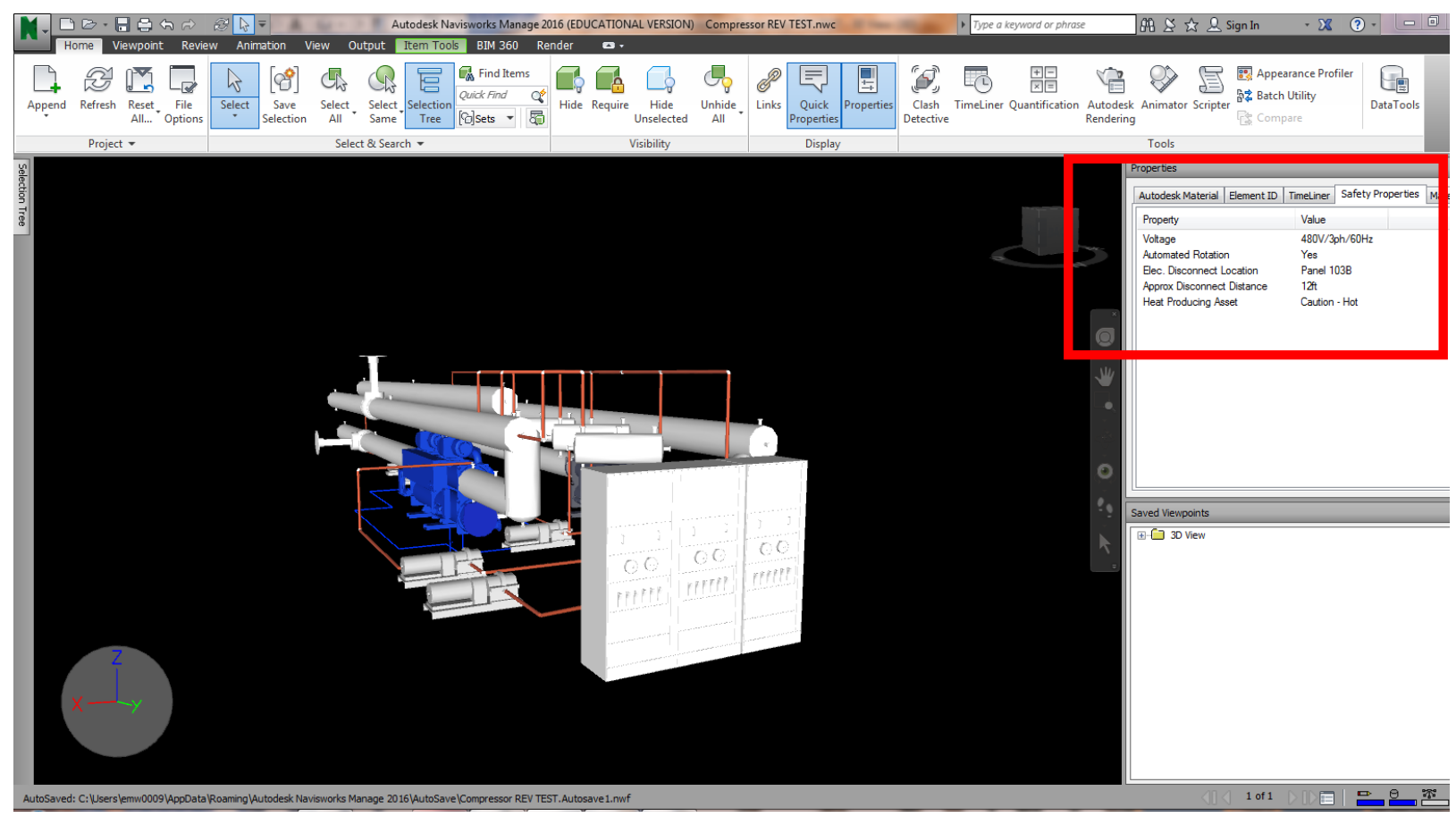

Figure 35: "Native BIM-Model” Inputs in Navisworks Manage 2016 
Beyond the "Native BIM-Model" inputs, and as additional safety inputs become available throughout the building's lifecycle, two additional mechanisms of data transfer can be utilized. "Non 3D" and "Direct Input" data inputs are both represented within this phase and are executed using one of two data transfer mechanisms:

1. Add New User Data Tab in Navisworks 2016

2. Using Selection Inspector and DataTools to add data externally in an Excel file and reimport into the model.

Although both data transfer mechanisms result in the same comprehensive, safety data loaded model, the selection of which mechanism is important for efficiency and organization. The use of Add New User Data Tab is most efficient when inputting safety inputs for a single, standalone piece of equipment. This scenario is likely when FM staff install a new or updated piece of equipment and the safety inputs need to be added to the graphical element. Using Selection Inspector and DataTools is utilized when bulk additions or inputs are required for a system. This transfer mechanism is also recommended for most "Non 3D" data inputs as the information is likely in various formats (.doc, .pdf, .xls, etc.) when being submitted to the FM entity.

\subsubsection{1. $\quad$ Add New User Data Tab}

Once appended into the Navisworks model, additional relevant safety data that was not input into the native model, can be added using Properties > Add New User Data Tab. The use of Add New User Data Tab is a viable solution for adding information to individual pieces of equipment. For bulk data loading, the utilization of DataTools and Selection Inspector is recommended. To add a new tab, right click in the properties space and Add New User Data Tab. This creates a blank tab titled "User Data." Right clicking in this new tab allows the user to Rename Tab and Insert New Property. Figure 36 presents the new safety property input into the compressor package. 


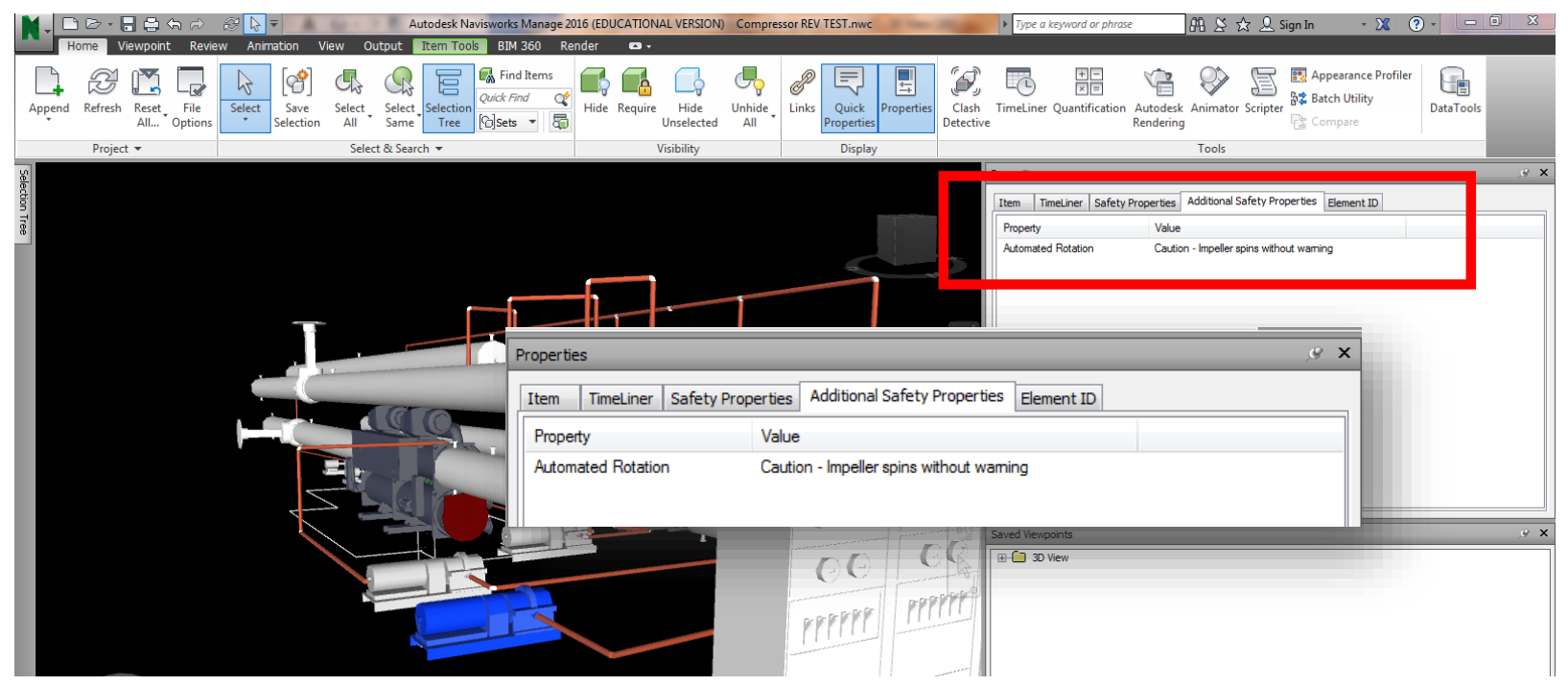

Figure 36: User Defined Safety Property Using “Add New User Data Tab”

\subsubsection{2. $\quad$ Selection Inspector and Data Tools}

The utilization of Selection Inspector and DataTools within Navisworks is a viable option to add or adjust multiple properties to a number of graphical elements. This process, although more labor intensive than previously presented processes, allows for data loading of multiple graphics. For this proof of concept model, a typical concern within a compressor room is the significant environmental decibel level that is generated when the compressor system is running. Because of this, hearing protection is required in the compressor room. In order to add this safety property to the environment (room), all pieces of equipment will inherent this safety property. By doing this, the safety property will be presented regardless of the user's equipment selection.

In order to add additional safety properties or adjust existing user defined properties to each piece of equipment, Selection Inspector and DataTools are utilized export and re-import safety information. Selection Inspector allows for the filtering of specific properties for selected pieces of equipment. This step is important in order to identify which properties a user wants to adjust or place into a new properties tab. DataTools is utilized in order to re-import the added/adjusted information back into the graphical model. Figure 37 presents the relevant information that needs to be exported using Selection Inspector for this proof of concept, while Figure $\mathbf{3 8}$ shows the added information in the exported CSV file. It is important to note that this type of data manipulation requires Microsoft Office 64-bit. 
- Property: Environment Decibel Level(dBA)

- Value: 105

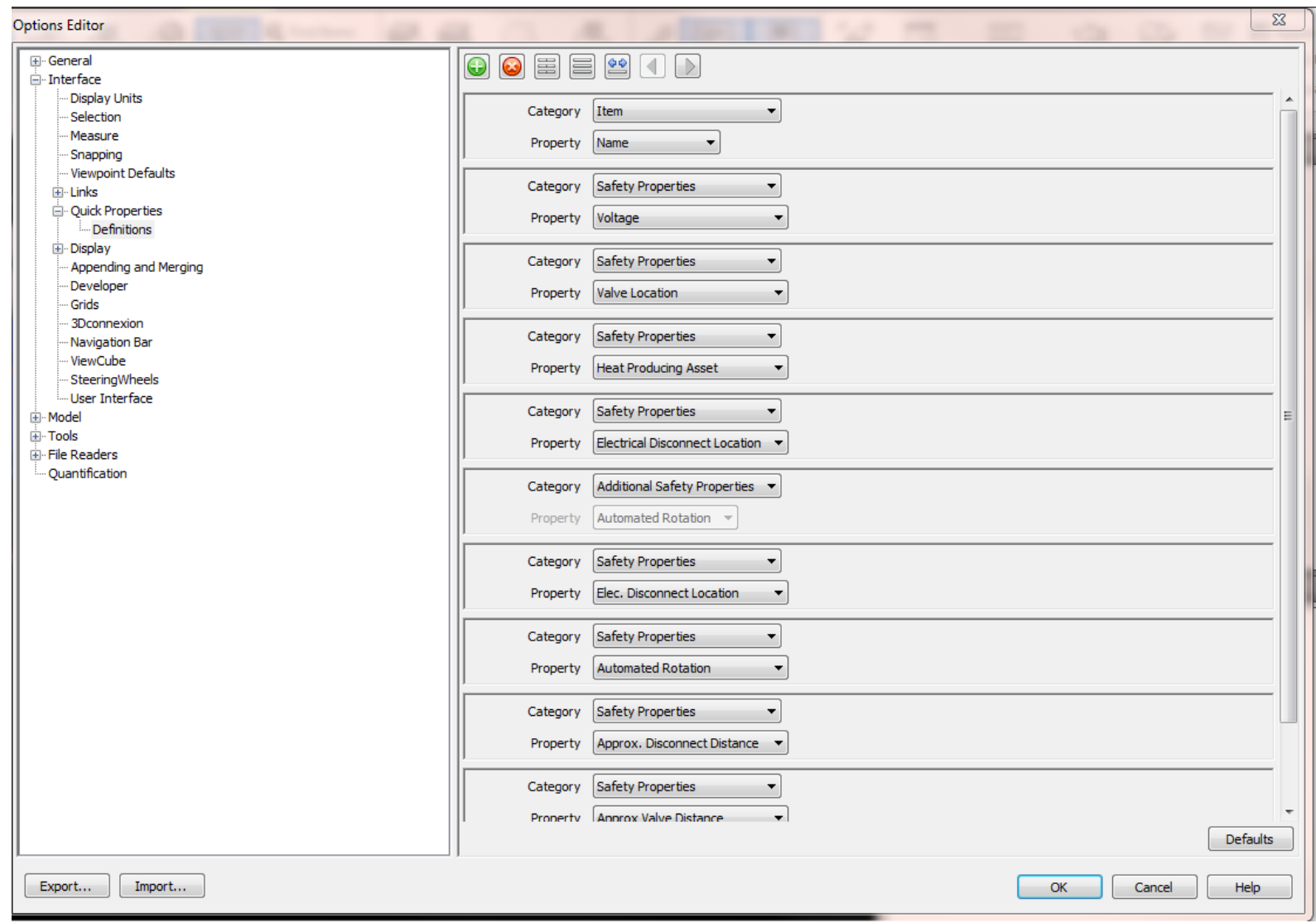

Figure 37: Selection Inspector Export 


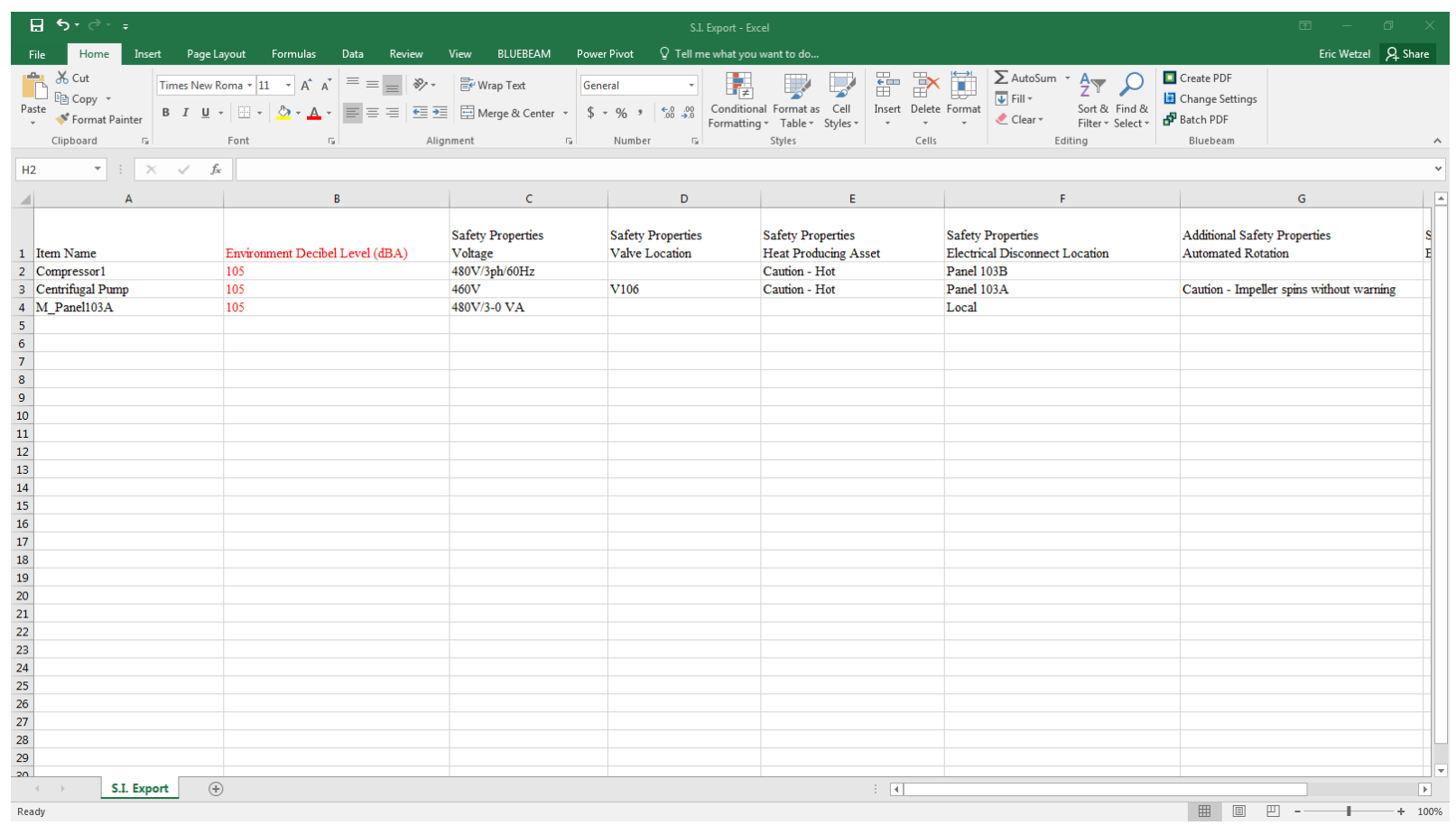

Figure 38: Exported Safety Information and Added “Environment Decibel Level (dBA)” Data

Once the new information is added to the Excel Worksheet, the file needs to be saved as an XLS. This needs to be done in order to reimport using DataTools. At this stage, Navisworks should be reopened and Data Tools launched. A new DataTools Link needs to be added. Figure 39 presents the information that must be added in order to map the Excel file to the graphics of the Navisworks file.

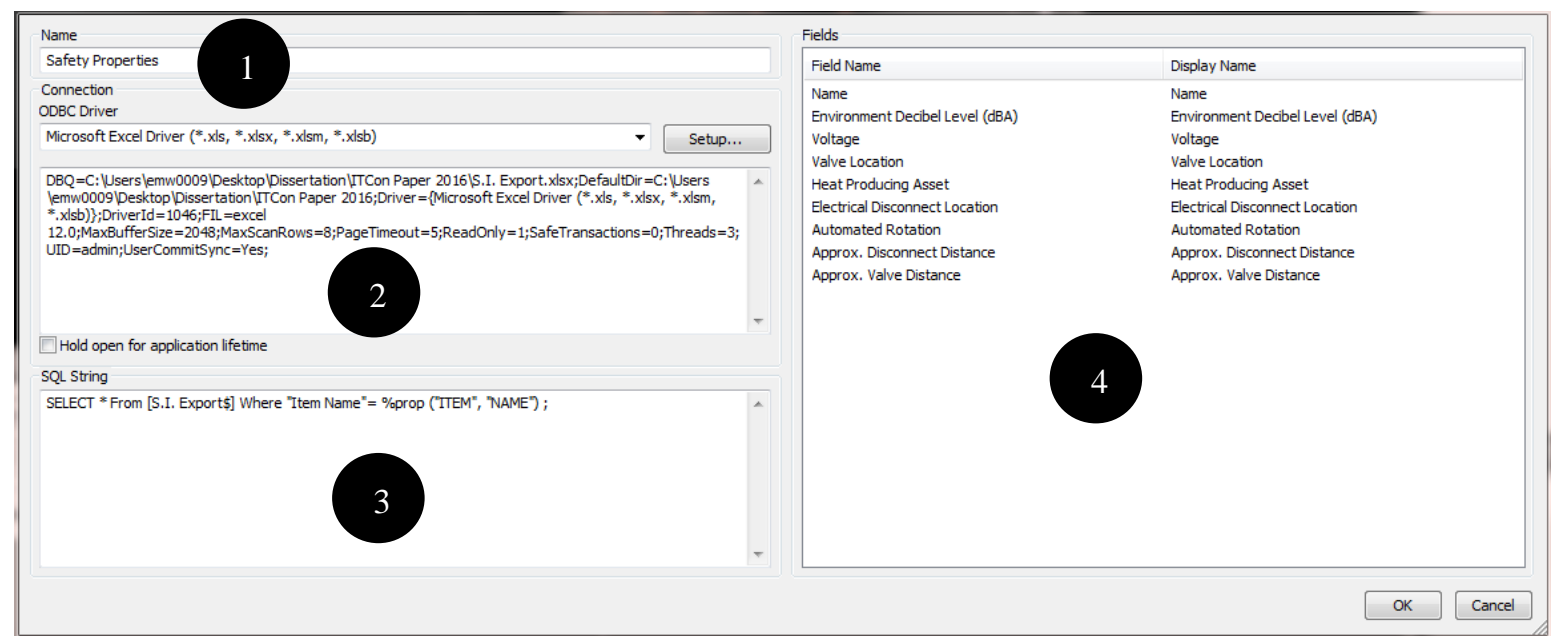

Figure 39: Data Tools Information 
1. A name is given to the new DataTools Link. This name will become the name of the properties tab in Navisworks that includes all of the properties identified in the "Fields" area.

2. Using an ODBC Driver connection, a link is made between the Excel file and the Navisworks file. Upon selecting the file in Setup. The mapping of the two files is automatically generated.

3. An SQL String needs to be written in order to link the information in the Excel file to the applicable graphics in the Navisworks file. This is executed by mapping the specific equipment name, known as "Item Name" in Navisworks, to the "Item Name" column in Excel. The SQL String reads as follows:

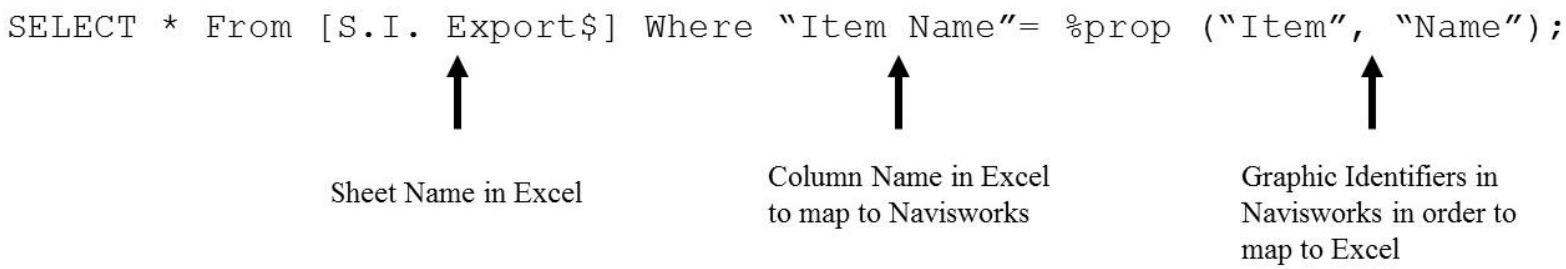

4. User needs to input the Field Name they wish to see under the new developed "Safety Properties" tab. For this example, the "Name" of the piece of equipment and the added "Environment Decibel Level" property will be mapped to the graphics. Only properties that have values will be transferred to individual graphics. Figure $\mathbf{4 0}$ presents the added safety properties in the newly developed "Safety Properties" tab for the electrical panel upon the execution of the DataTools process. 


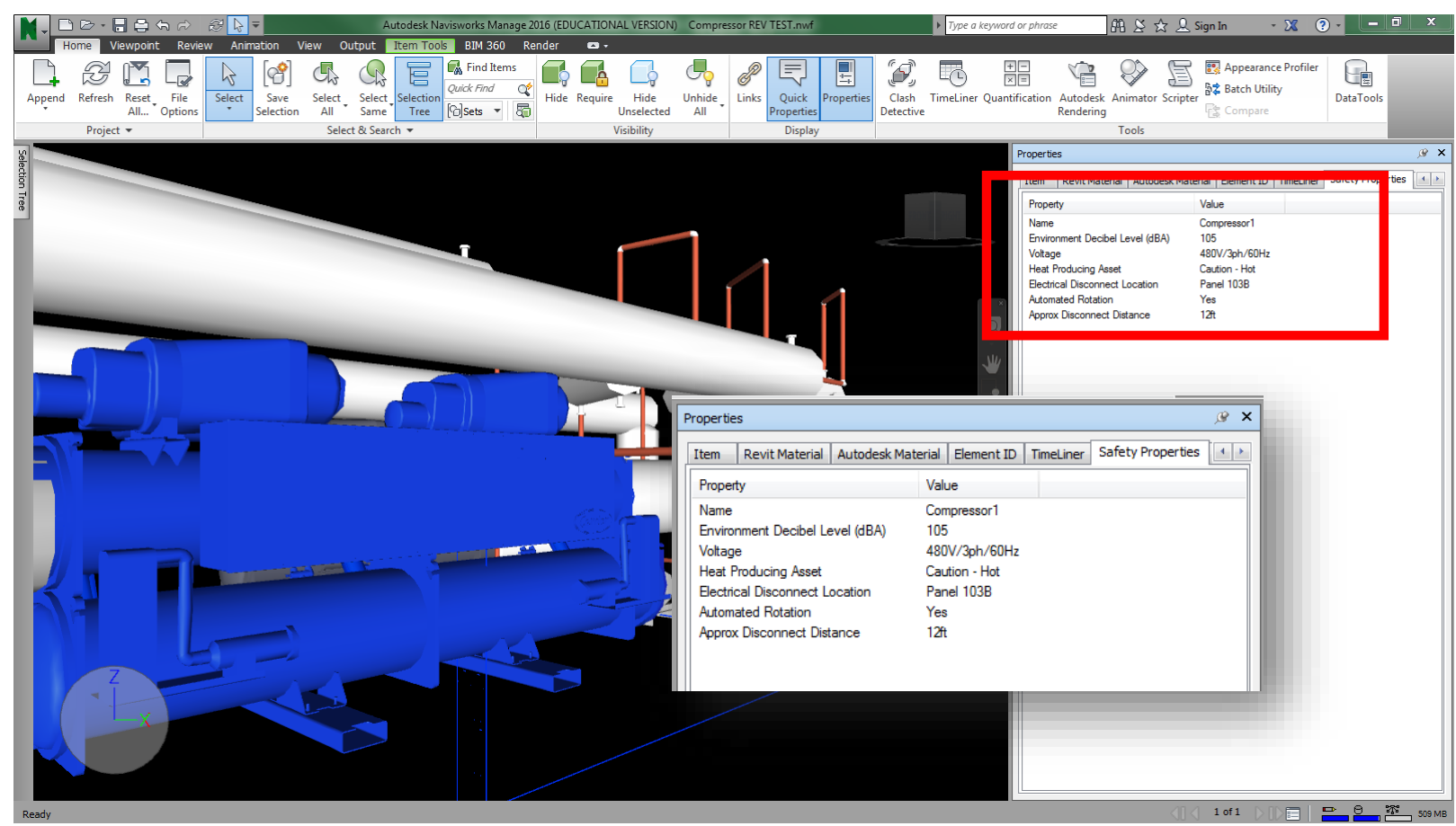

Figure 40: Added Safety Properties Under New "Safety Properties" Tab

This process can be repeated to add or update information. Once all the relevant safety information is stored in the Integrated BIM Model, launching the DRPS adds logic and guides the information exchange of the safety data. As described in Wetzel and Thabet (2015), "Based on the values assigned to the properties, rules and process models guide the information logic and present the information via a graphical user interface (interface). 


\section{DATA RETRIEVAL AND PROCESSING SYSTEM (DRPS)}

Once all the relevant safety information is stored in the Appended BIM Model, launching the DRPS adds logic and guides the information exchange of the safety data. Figure 41 identifies this final step within the BIM-Based Safety Framework as Phase III.

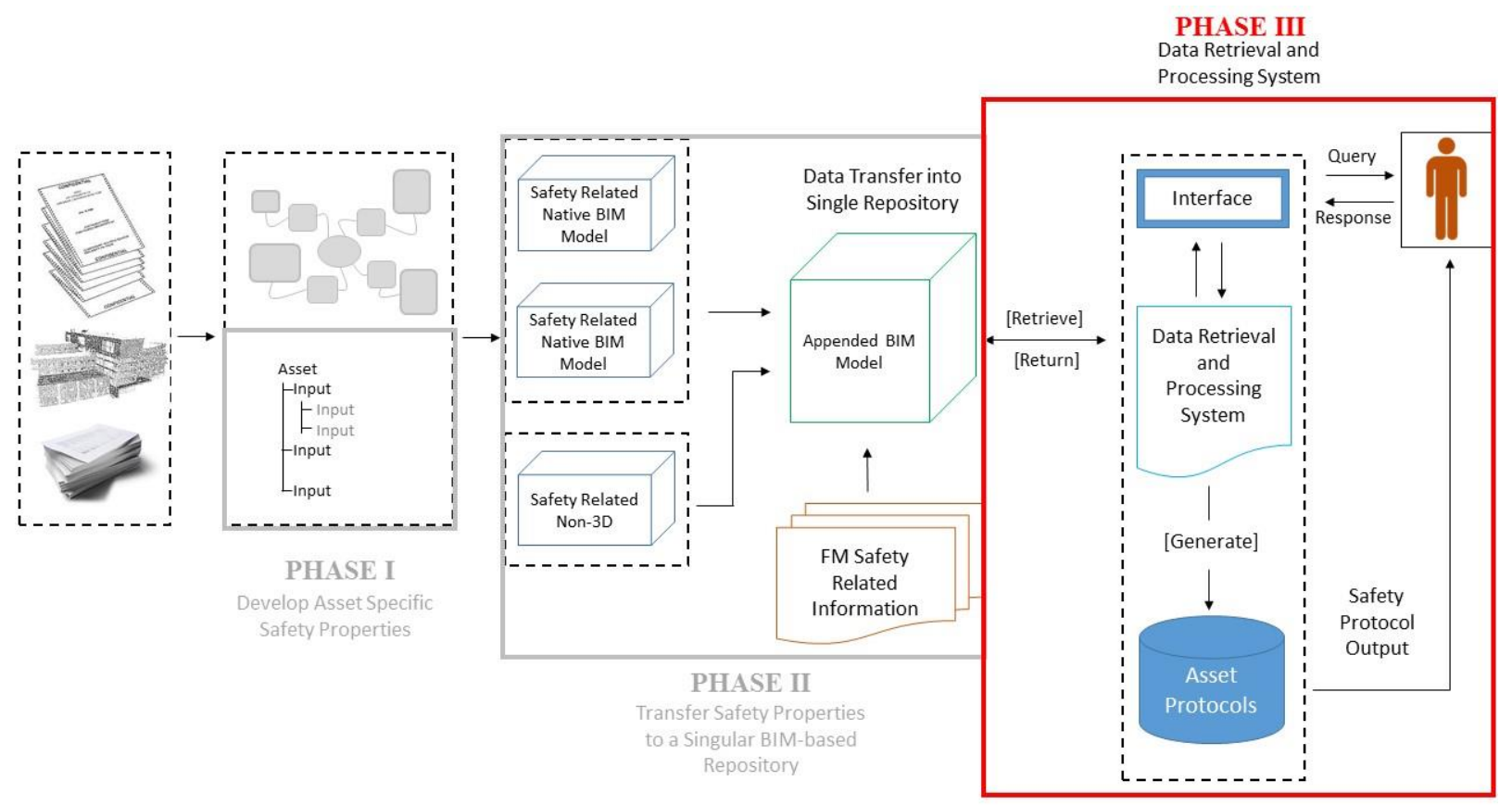

Figure 41: Phase III of the BIM-Based Safety Framework

The DRPS has two primary functions. First, retrieve the asset specific safety inputs and values stored within the Appended BIM Model. Accessing the data is the first step in order to enact the data processing on the returned results. The second function of the DRPS is to recognize FM tasks that require user input and subsequently launch the query system. In order to properly present the safety hazards and appropriate protocols to the FM users, the decision making of the user must be taken into account. Although many of the safety hazards and protocols can be presented to the user based strictly on the asset being maintained or the environment in which the asset resides, the FM user will make decisions that will impact the safety of the FM task. An example of this is the selection of a lift system in order to execute a task at elevation. The protocols regarding work on a ladder differ significantly from working on a man lift. In order to present task-specific safety information to a FM worker, all of the factors regarding the asset, environment, and approach must be known. Other examples of task specific queries are depicted in Table 26. This list is nonexhaustive and while some standard queries can be loaded into the system, in a commercial application, the task specific queries within the DRPS would need to be customized to the particular facility. 
Table 26: Task Specific Queries Launched by the DRPS (Examples)

\begin{tabular}{|l|l|l|}
\hline Safety Input(s) & \multicolumn{2}{l|}{ Protocol } \\
\hline $\begin{array}{l}\text { Maintenance Requires a Lift } \\
\text { System }\end{array}$ & $\begin{array}{l}\text { Identify the lift system utilized } \\
\text { for this FM task: <list available } \\
\text { lift system> }\end{array}$ & Lift system specific \\
\hline Voltage & $\begin{array}{l}\text { Yes or No, this FM activity } \\
\text { requires exposing live current? } \\
\text { <radio button selection> }\end{array}$ & Lockout/Tagout \\
\hline $\begin{array}{l}\text { Environmental Air Quality } \\
\text { Hazards Present }\end{array}$ & $\begin{array}{l}\text { Yes or No, this FM task } \\
\text { requires welding? <radio button } \\
\text { selection> }\end{array}$ & $\begin{array}{l}\text { Welding in toxic, gaseous, } \\
\text { chemical, and/or enriched } \\
\text { environments }\end{array}$ \\
\hline $\begin{array}{l}\text { Maintenance Requires Lift } \\
\text { System + Outdoor Environment } \\
+ \text { Mechanized Lift System } \\
\text { Selection }\end{array}$ & $\begin{array}{l}\text { Yes or No, the current wind } \\
\text { speed is within the specified } \\
\text { safety considerations for this } \\
\text { lift system? <radio button } \\
\text { selection> }\end{array}$ & Lift system specific \\
\hline
\end{tabular}

Retrieving stored asset information, analyzing that information, and ultimately presenting the applicable, task-specific protocols to the FM worker is the primary objective of the DRPS. This chapter will present the system architecture required to execute this objective, validate the system architecture through various FM test cases, and present a proof of concept by presenting the conceptual GUI within the sequence diagrams in the context of a selected test case.

\subsection{System Architecture}

In order to present the functionality of the DRPS, three platforms are utilized - a product model, a sequence diagram, and a conceptual GUI. A product model uses an object-oriented data structure to formally classify information to support the exchange of data through a mechanism (Eastman 1999). By executing a product model through a UML Class Diagram, a type of "Structural Diagram," a conceptual schema or framework of data can be properly structured and stored (Pilone and Pitman 2005). A sequence diagram, a type of "Behavioral Diagram," presents how objects interact in a particular scenario over a period of time (Visual Paradigm 2016). For this research, a sequence diagram is developed to present how the product model classes interact and the system retrieves the relevant safety information. Finally, a conceptual GUI is utilized in order to present the interface between the user and the DRPS. The use of a conceptual GUI in correlation to the product model and sequence diagram in the context of a test case presents a proof concept. 


\subsubsection{Product Model and Sequence Diagram Development}

The product model (UML class diagram) in this research is developed based on the information obtained through the first two phases of the Six Sigma methodology - Define and Measure. Establishing what safety information is relevant to FM workers and how that information is related, sets the baseline for the product model development. Figure 42 presents the UML class diagram for the DRPS.

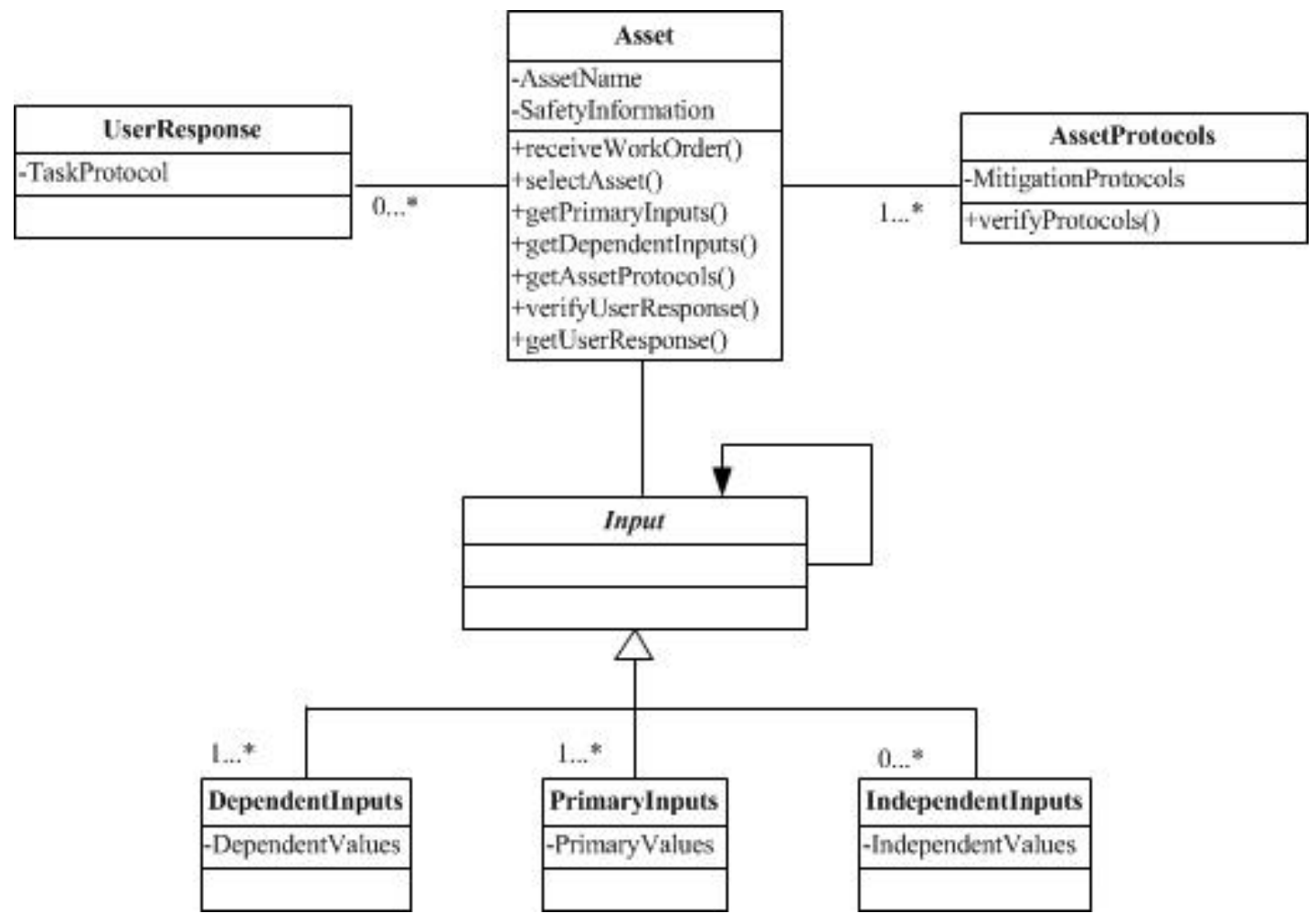

Figure 42: DRPS Product Model

The developed product model uses a single core class and five subclasses in order to execute delivery of safety information. As set, the core class, is a robust class where the initial event takes place and is required to distribute tasks, obtain the returned information, verify additional user input, and present the comprehensive safety information. DependentInputs, PrimaryInputs, and IndependentInputs, are all dependent classes to the abstract class Input. The Input class is a subclass to Asset and has no operation. The Input subclass holds the properties and values for each stored asset. UserResponse stores the task specific inputs that may or may not return a value. These inputs are presented only when a query system response is required. The final subclass, AssetProtocols, maintains the hazard mitigation techniques for each input. 
As shown in the product model, each class serves a function in the system architecture. In order to present how the classes interact within the system over a period of time, a sequence diagram is developed. The sequence diagram (Figure 43) graphically depicts the steps that the DRPS utilizes from receipt of an event to the delivery of the safety information.

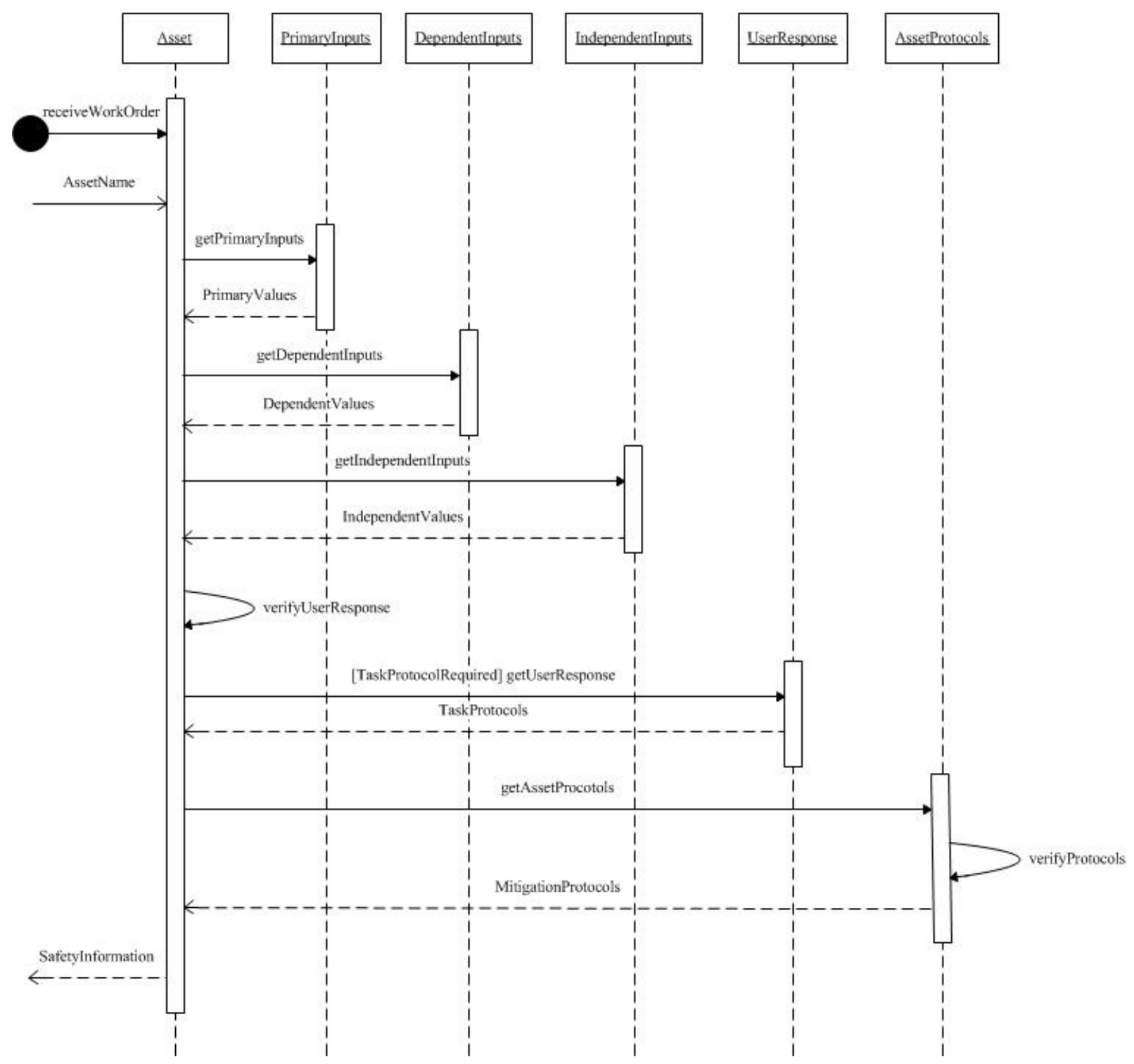

Figure 43: DRPS Sequence Diagram

The initiation event in a FM task is often the receipt of the work order with an asset requiring maintenance. Within the sequence diagram, this event prompts the user to select which Asset is requiring maintenance. Upon receipt of the Asset, the system can retrieve the stored PrimaryInputs, 
DependentInputs, and IndepentdentInputs for that specific asset. Once all the safety inputs are returned, the system will call on itself to VerifyUserResponse. By evaluating the returned inputs, the system will check to see if additional task specific information is required by the user. For example, the selection of a lift system. The UserResponse is not required in all FM tasks and therefore is guarded by [TaskProtocolRequired]. If the specific task does require task specific information from the user, the system will initiate the query system and retrieve the responses. With all of the inputs returned, the system can retrieve the applicable AssetProtocols. Before returning the protocols, the system will once again call on itself to evaluate the information through VerifyProtocols. This function verifies the retrieved protocols and executes the following tasks if required:

- Eliminate Protocol Redundancies - As shown in Table 20 a number of Safety Inputs share Safety Protocols (e.g. Hazardous Energy \& Automated Crushing/Slicing/Rotating Mechanism). In order to eliminate congestion or confusion in the output to the user, the DRPS will eliminate redundancies of returned protocols and only present the information once.

- Eliminate Non-Applicable Protocols Based on User Response - Each Safety Input has a set of Safety Protocols that should be delivered if a value is available for the DRPS to return. In most cases this functionality works without intervention from the DRPS; however, in some instances a user response will require a decision to be executed by the DRPS in order to present the correct protocol.

For example, the user selects "Ladder" as their lift system. Utilizing a ladder does not require the use of a fall arrest system and therefore this protocol can be eliminated from the returned MitigationProtocols. The script for this function is as follows:

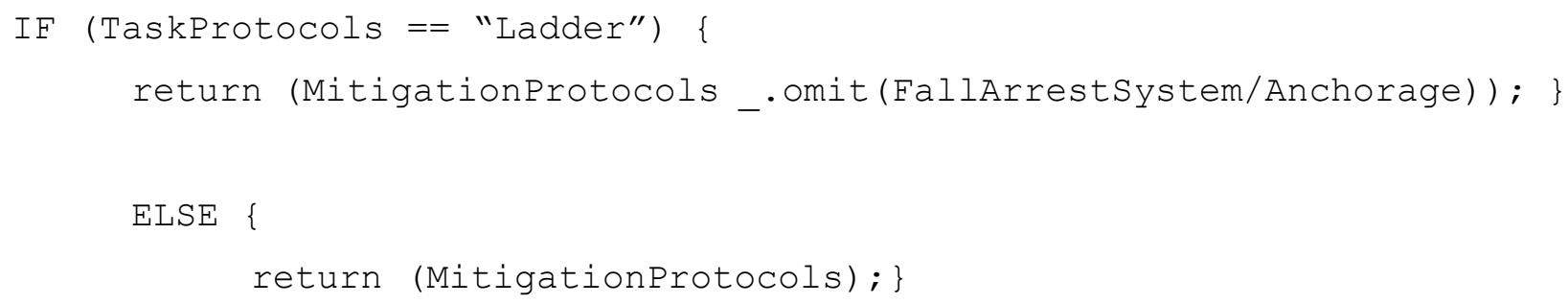

The above rule states that if the TaskProtocols selected by the user is "Ladder," the system will omit the FallArrestSystem/Anchorage protocol. This function is required as a fall arrest system isn't required while utilizing a ladder. With any other UserResponse (manlift, scissor lift, platform, etc.) the system will return the full array of MitigationProtocols identified by the system. 
Once the system verifies the protocols, the MitigationProtocols are sent back to the asset and presented as structured SafetyInformation for user consumption. The SafetyInformation in this research plays an important role in the DRPS system as a mechanism for presenting the protocols to the user; however, the scope of this research excludes the media in which this information is presented. Currently, the system conceptually presents this information as text-based listing of safety hazards and protocols. As shown in Chapter 8, future research may identify a better mechanism to present this information (e.g. videos, images, augmented reality, etc.).

Chapter 7 System Architecture Validation presents the last phase of the Six Sigma methodology, Verify, through a number of mechanisms to test the system functionality. 


\section{SYSTEM ARCHITECTURE VALIDATION}

As with the development of any new process, validation through an existing evaluation mechanism is important. To ensure that the product model design and sequence diagram executed within this research elected to validated the system based on Turkaslan-Bulbul and Akin (2007). This validation step requires that the product model maintain three characteristics:

1. The Scope: the information covered in the data model is capable of representing the attributes of the domain.

2. The Flexibility: the data model is designed to have a modular structure, where new data can be added or exchanged within the system without major edits.

3. The Support: the functional usage of the product model.

The first characteristic, scope, has already been validated through the execution of the Data Validation interviews. As the product model was developed from the validated data points collected within this research, the attributes of the product model are confirmed as domain specific. The flexibility of the product model and sequence diagram will be validated through FM test cases. By back-checking the product model and sequence diagram on various scenarios utilizing multiple safety inputs, nuances to each scenario can be incorporated in order to validate the flexibility of the system. If the product model and sequence diagram is robust enough to not require major edits through all the test cases, the system is validated. Finally, the functional nature of the product model and sequence diagram is validated through the incorporation of the conceptual GUI. By presenting the product model and sequence diagram in the form of a graphical interface, the industrial application or functional usage of the system is presented. Additionally, this step will serve as a proof of concept for the DRPS.

The following test cases are utilized in order to verify that the product model and sequence diagram developed in this research function as intended. Each test case will present a unique FM task and associated hazards. Running the system through multiple scenarios validates the architecture of the system. The test cases utilized in this section are a combination of two fatal accidents from the FACE reports and two typical FM tasks validated by industry experts. The structure of each section is as follows:

1. The task being performed or the accident that occurred.

2. The data loaded ASIT as it would be presented for the specific asset requiring maintenance.

3. Table summarizing the ASIT data load.

4. The sequence diagram with the applicable commands being executed based on the FM test case. 
Following the test cases, a proof of concept is presented in Section 7.4 DRPS Proof of Concept by incorporating one of the FM test cases with the conceptual graphical user interface.

\subsection{FM Test Case No. 1 - FACE Report 9013}

A 21-year-old worker died as a result of injuries sustained in a 12-foot fall from a scaffold. The victim was a member of a six-man crew engaged in the removal of asbestos-contaminated insulation from a series of large ducts on the exterior of an electric power generation plant. The victim was removing asbestos insulation from a large outdoor metal duct approximately 14 feet above the ground. The worksite was accessed by tubular metal scaffolding. The victim was working at the 12-foot level of the scaffold. The scaffold was not decked at this level. Instead, the crew had installed a single 2-inch by 12-inch plank across the tubing. The plank extended beyond the tubing on both sides and was not fastened in position to the tubing. Instead, the crew had driven two nails into each end of the plank at 45 degree angles to hold the plank against the tubing while allowing them to slide the plank along the tubing to various areas where they were working. The nails on one end of the plank had loosened sufficiently to slip free from the scaffold. The weight of the victim on the opposite end of the plank caused the plank to rise up in the air, dropping the victim to the ground below.

Based on the above FM test case, values can be assigned to the ASIT identified safety properties for asset group Ducts. Figure $\mathbf{4 4}$ presents the data load applicable for this specific FM test case. Note: Not all of the necessary safety data presented in the ASIT is available in the FACE Report but has been added in order to present the appropriate data loading for this test case. 


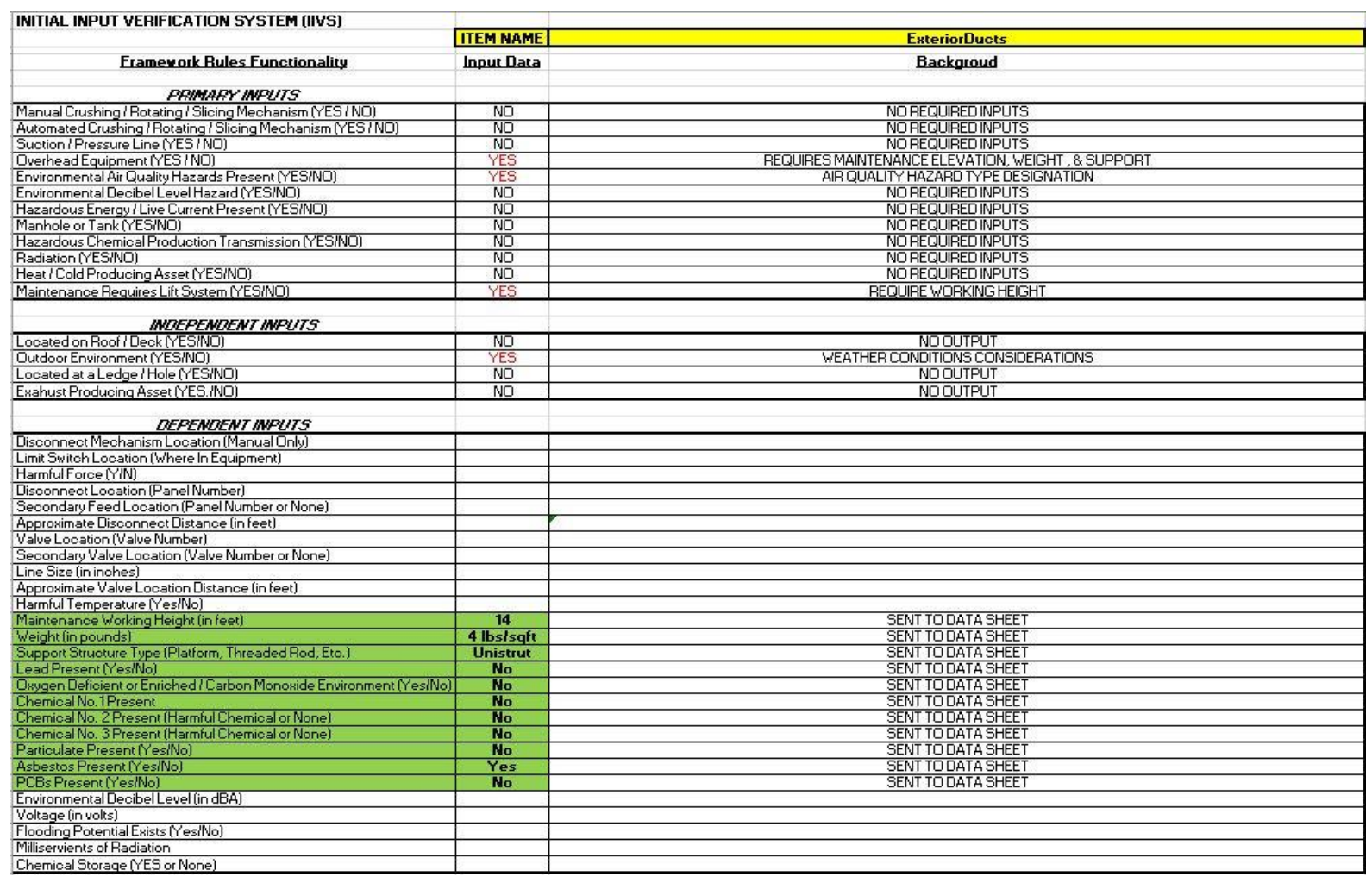

Figure 44: ASIT Data Load for Ducts - FM Test Case No. 1

Table 27 presents the values required for the Ducts for this particular test case.

Table 27: Test Case No. 1 Relevant ASIT Data for ExteriorDucts

\begin{tabular}{|c|c|c|}
\hline \multicolumn{1}{|l|}{ Input Type } & Overhead Equipment & Yes \\
\hline Primary & Maintenance Working Height & $14 \mathrm{ft}$ \\
\hline Dependent & Weight & 4 lbs/sqft \\
\hline Dependent & Support Structure Type & Unistrut \\
\hline Dependent & Maintenance Requires Lift System & Yes \\
\hline Primary & Maintenance Working Height & $14 \mathrm{ft}$ \\
\hline Dependent & Environmental Air Quality & Yes \\
\hline Primary & Asbestos Present & Yes \\
\hline Dependent & Outdoor Environment & Yes \\
\hline Independent & & \\
\hline
\end{tabular}

Figure 45 outlines the process the DRPS would execute in order to return results to the FM worker prior to executing the FM task. 


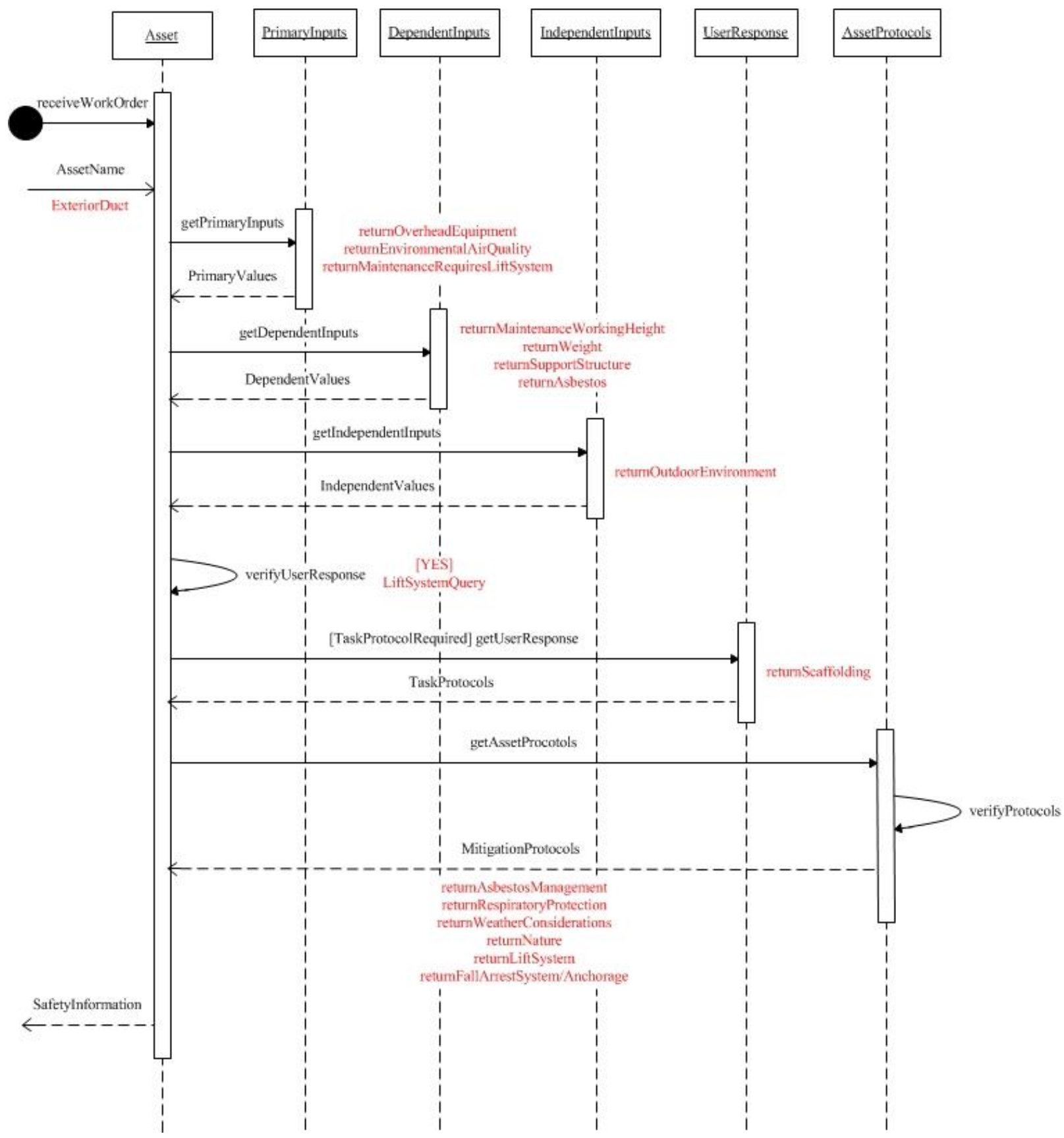

Figure 45: Sequence Diagram for Ducts - FM Test Case No. 1

Upon receiving the work order and launching the DRPS, the system will identify the Asset and execute the getPrimaryInputs, getDependentInputs, and getIndependentInputs command. Executing these commands for the asset requiring maintenance returns the PrimaryValues (OverheadEquipment, EnvironmentalAirQuality,

$\&$ 
(MaintenanceWorkingHeight, Weight, SupportStructure, \& Asbestos), and the IndependentValues (OutdoorEnvironment). Once the DRPS returns all of the applicable values, the system can process the information and verify if a query should be launched. In this scenario, the MaintenanceRequiresLiftSystem input has been assigned a value and therefore the system will query the user on which lift system will be utilized. From the FM test case an election of Scaffolding is made. With all of the inputs identified, the system can evaluate and return the MitigationProtocols for the specific FM task. The protocols being returned in this test case address three hazards:

1. Asbestos

a. returnAsbestosmanagement
b. returnRespiratoryProtection

2. Outdoor Environment

a. returnWeatherConsiderations

b. returnNature

3. Falls

a. returnLiftSystem (Scaffolding Protocol)

b. returnFallArrestSystem/Anchorage

\subsection{FM Test Case No. 2 - Coal Fired Boiler Preventative Maintenance}

The following test case was validated by a Safety Compliance Officer at a university located in the Eastern USA.

An FM worker is tasked with cleaning the fire box of a 7'x7' coal-fired boiler. As the boiler burns coal in order to produce steam, ash and soot lines the interior of the fire box. As ash and soot build up within the boiler, the efficiency of the boiler is reduced. Preventative maintenance protocols require the fire box be cleaned biennially. The boiler requiring maintenance is located within one of the power houses on the campus where a number of motors and tanks create a high decibel level environment. In order to thoroughly clean the boiler fire box the worker must enter the tank which has been identified as a confined space.

Based on the above FM test case, an ASIT can be loaded with the applicable safety information. Figure 46 presents the data load applicable for this FM test case. 


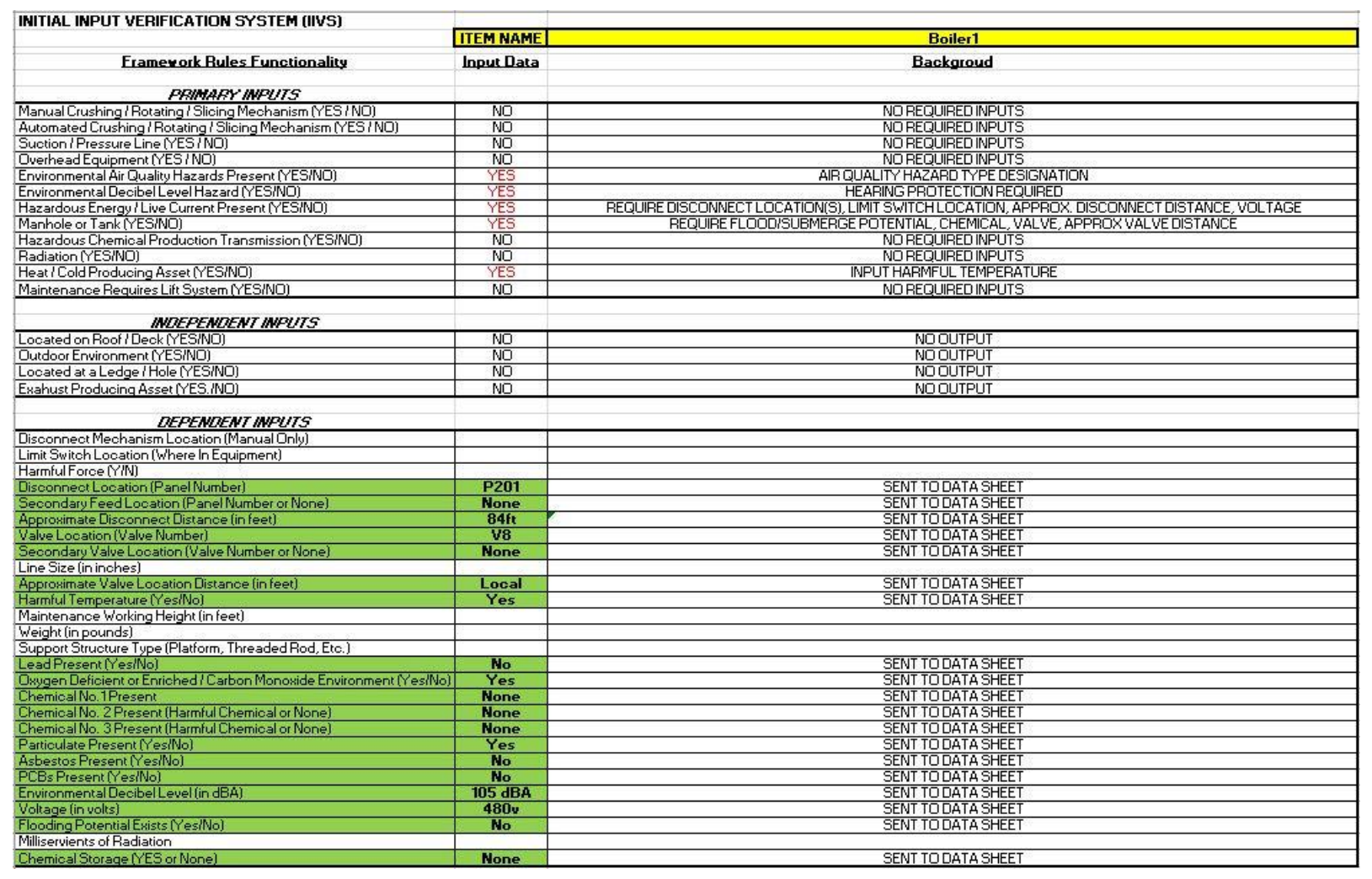

Figure 46: ASIT Data Load for Boiler1 - FM Test Case No. 2

Table 28 presents the values required for the Industrial Coal-fired Boiler for this particular test case. 
Table 28: Test Case No. 2 Relevant ASIT Data for Boiler1

\begin{tabular}{|c|c|c|}
\hline Input Type & Inputs & Yes \\
\hline Primary & Environmental Air Quality & Yes \\
\hline Dependent & Oxygen Deficient/Enriched/CO Env. & Yes \\
\hline Dependent & Particulate Present & Yes \\
\hline Primary & Environmental Decibel Level & $105 \mathrm{dBA}$ \\
\hline Dependent & Environmental Decibel Level (in dBA) & Yes \\
\hline Primary & Hazardous Energy/Live Current Present & P201 \\
\hline Dependent & Disconnect Location & $84 \mathrm{ft}$ \\
\hline Dependent & Approximate Disconnect Distance & $480 \mathrm{v}$ \\
\hline Dependent & Voltage & Yes \\
\hline Primary & Manhole / Tank / Confined Space & V8 \\
\hline Dependent & Valve Location & Local \\
\hline Dependent & Approximate Valve Distance & Yes \\
\hline Dependent & Harmful Temperature & Yes \\
\hline Primary & Heat/Cold Producing Asset & Yes \\
\hline Dependent & Harmful Temperature & . \\
\hline
\end{tabular}

Figure 47 outlines the process the DRPS would execute in order to return results to the FM worker prior to executing the FM task. 


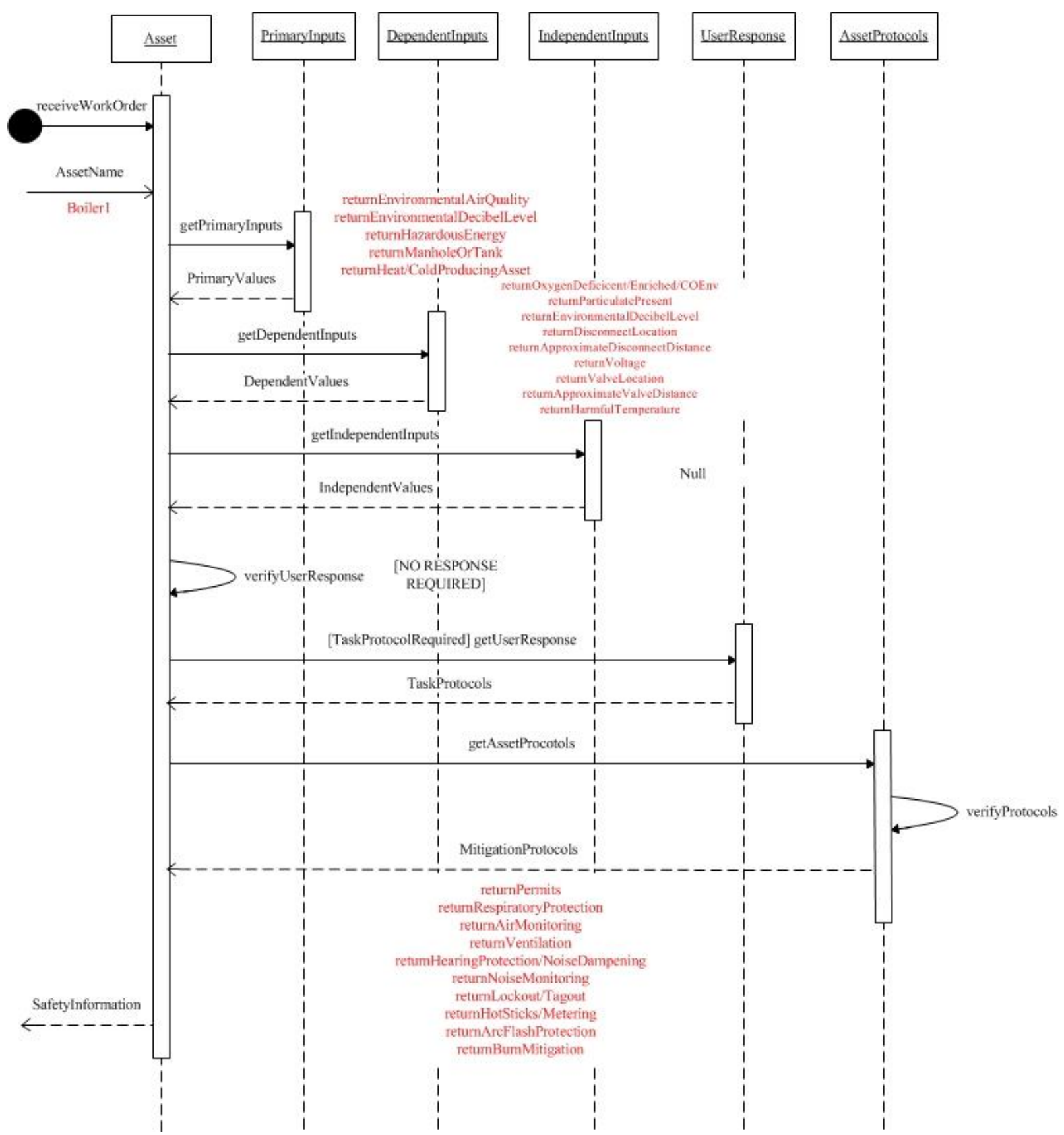

Figure 47: Sequence Diagram for Boiler1 - FM Test Case No. 2

Upon receiving the work order and launching the DRPS, the system will identify the Asset and execute the getPrimaryInputs, getDependentInputs, and getIndependent Inputs command. Executing these commands for the asset requiring maintenance returns the PrimaryValues (EnvironmentalAirquality, EnvironmentalDecibellevel, HazardousEnergy, Manhole/Tank/ConfinedSpace, and Heat/ColdProducingAsset), the DependentValues (OxygenDeficient/Enriched/COEnvironment, 
ParticulatePresent, EnvironmentalDecibelLevel, DisconnectLocation, ApproxiamteDisconnectDistance, Voltage, Valvelocation, ApproximateDisconnectDistance, HarmfulTemperature). Once the DRPS returns all of the applicable values, the system can process the information and verify if a query should be launched. In this scenario, no query system launch is required and therefore the getUserInput action is bypassed. With all of the inputs identified, the system can evaluate and return the MitigationProtocols for the specific FM task. The protocols being returned in this test case address five hazards:

1. Environmental Air Quality

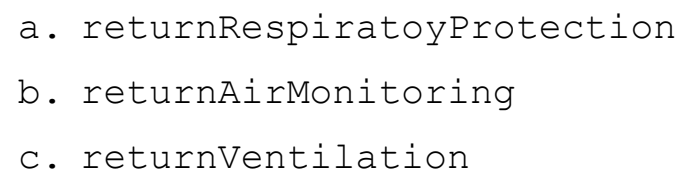

2. Environmental Decibel Level
a. returnHearingProtection/NoiseDampening
b. returnNoiseMonitoring

3. Hazardous Energy
a. returnLockout/Tagout
b. returnHotsticks/Metering
c. returnArcFlashProtection

4. Confined Space

a. returnPermits

5. Heat/Cold Producing Asset

a. returnBurnMitigation

\subsection{FM Test Case No. 3 - FACE Report 9717}

A 37-year-old male maintenance electrician (the victim) died when his lower torso was crushed between the nip barrier (a wire-mesh gate) and the upper frame of a paper rewinder machine at a paper manufacturing facility. Without first de-energizing, locking out, and tagging the machine, the victim began to replace the arm for the limit switch that controlled upward movement of the nip barrier. He climbed an 8-foot stepladder to access the top of the machine where the switch was located, and leaned into the 16inch opening between the top of the nip barrier and the upper frame of the machine. Co-workers observed him reaching with a screwdriver into the area where the switch was located. Apparently he inadvertently activated the limit switch and the nip barrier raised, carrying the victim and the stepladder upward and compressing both between the nip barrier and the upper frame of the machine. The victim's waist to lower 
back area was crushed. A co-worker paged the plant safety watchman, who contacted the rescue squad. The rescue squad arrived within 2 minutes, and the victim was pronounced dead at the scene.

Based on the above FM test case, an ASIT can be loaded with the applicable safety information. Figure 48 presents the data load applicable for this FM test case. Note: Not all of the necessary safety data presented in the ASIT is available in the FACE Report but has been added in order to present the appropriate data loading for this test case.

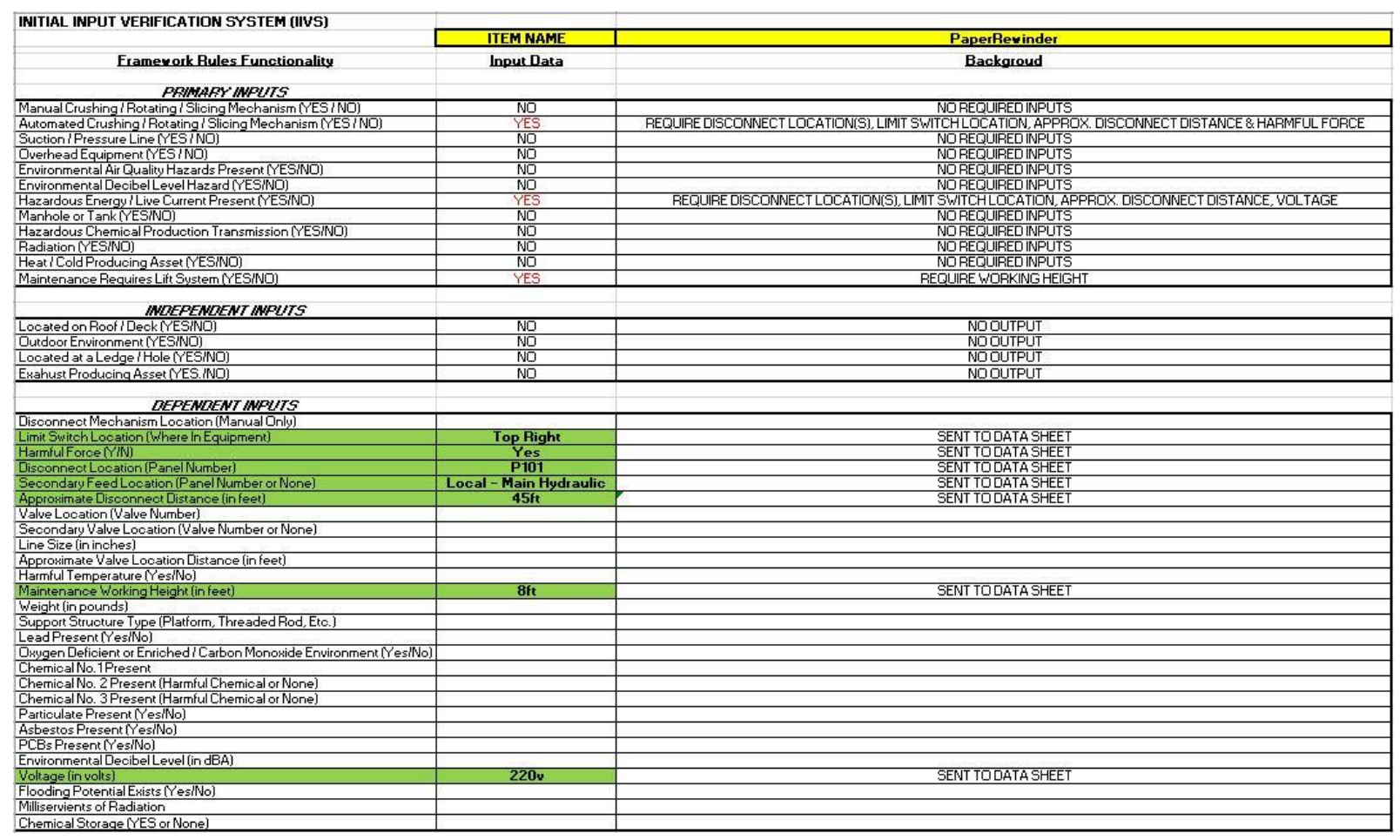

Figure 48: ASIT Data Load for PaperRewinder - FM Test Case No. 3

Table 29 presents the values required for the PaperRewinder for this particular test case. 
Table 29: Test Case No. 3 Relevant ASIT Data for PaperRewinder

\begin{tabular}{|c|l|l|}
\hline Input Type & Inputs & Value \\
\hline Primary & $\begin{array}{l}\text { Automated Crushing/Rotating/Slicing } \\
\text { Mechanism }\end{array}$ & Yes \\
\hline Dependent & Limit Switch Location & Yes \\
\hline Dependent & Harmful Force & P101 \\
\hline Dependent & Disconnect Location & Local - Main Hydraulic \\
\hline Dependent & Secondary Feed & $45 \mathrm{ft}$ \\
\hline Dependent & Approximate Disconnect Distance & Yes \\
\hline Primary & Hazardous Energy & $220 \mathrm{v}$ \\
\hline Dependent & Voltage & P101 \\
\hline Dependent & Disconnect Location & Local - Main Hydraulic \\
\hline Dependent & Secondary Feed & $45 \mathrm{ft}$ \\
\hline Dependent & Approximate Disconnect Distance & Yes \\
\hline Primary & Maintenance Requires Lift System & $8 \mathrm{ft}$ \\
\hline Dependent & Working Height & \\
\hline
\end{tabular}

Figure 49 outlines the process the DRPS would execute in order to return results to the FM worker prior to executing the FM task. 


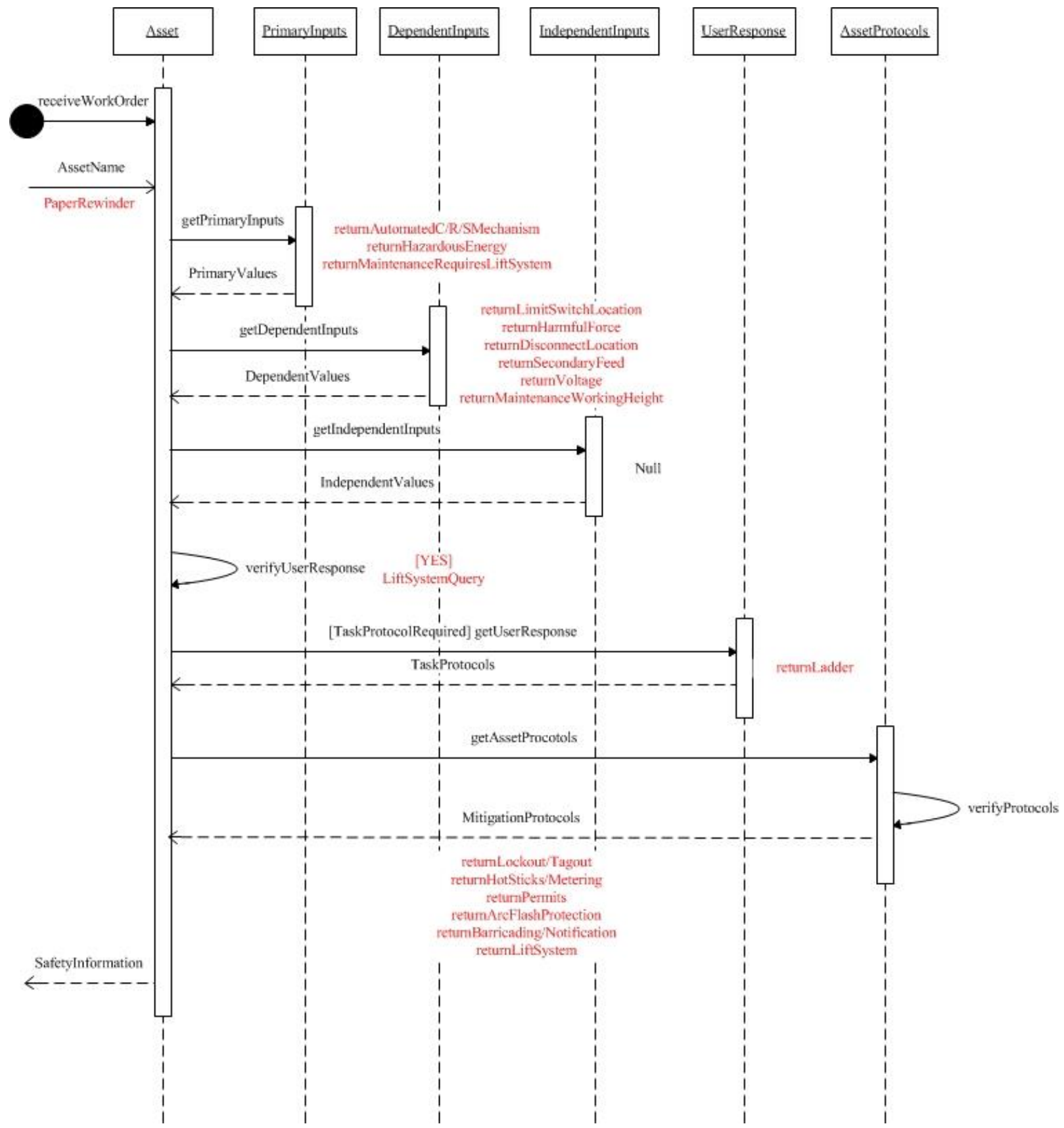

Figure 49: Sequence Diagram for PaperRewinder-FM Test Case No. 3

Upon receiving the work order and launching the DRPS, the system will identify the Asset and execute the getPrimaryInputs, getDependentInputs, and getIndependent Inputs command. Executing these commands for the asset requiring maintenance returns the PrimaryValues (AutomatedCrushing/Rotating/SlicingMechanism, HazardousEnergy, \& MaintenanceRequiresLiftSystem), the DependentValues (LimitSwitchLocation, Hamrfulforce, DisconnectLocation, \& SecondaryFeed, Voltage, 
MaintenanceWorkingHeight). In this test case, there are no IndependentValues and therefore the system will not return a value. Once the DRPS returns all of the applicable values, the system can process the information and verify if a query should be launched. In this scenario, the MaintenanceRequiresLiftSystem input has been assigned a value and therefore the system will query the user on which lift system will be utilized. From the FM test case an election of Ladder is made. With all of the inputs identified, the system can evaluate and return the MitigationProtocols for the specific FM task. The protocols being returned in this test case address three hazards:

1. Hazardous Energy

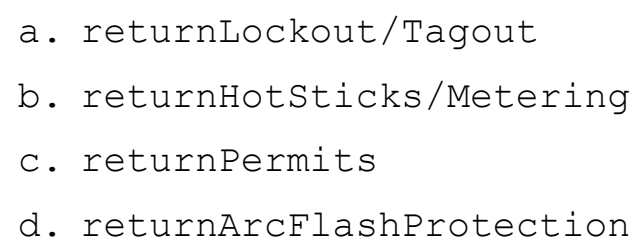

2. Automated Crushing/Slicing/Rotating Mechanism

a. returnBarricading/Notification

3. Falls

a. returnLiftSystem (Ladder Protocol)

The above test case represents the parent asset group (PaperRewinder) and all safety inputs requiring values are present.

\subsection{FM Test Case No. 4 - Liquid Anhydrous Ammonia \& Oil Separation}

The following test case was validated by an Operations Manager and Arena Technician at a large civic center (with ice rink) in the Southeastern USA.

A FM Worker at an ice skating rink needs to maintain the ammonia tanks that cool a brine solution required for the sheet of ice. The rink utilizes an indirect brine system in order to facilitate heat exchange. The brine solution runs through the ice floor, absorbing the floor's heat and keeping the ice sheet cool. When the brine solution returns to the compressor system, it is run through coils housed in a tank filled with ammonia in order to draw the heat out of the brine solution, cooling the solution to be sent back to the ice rink floor. The ammonia compressor in the system utilizes self-lubrication in order to get oil to the mobile parts of the compressor. Over time, the oil seeps from the compressor crankcase and becomes mixed with the liquid anhydrous ammonia. In order to maintain the thermodynamic efficiency of the ammonia surrounding the brine coils, the oil and ammonia mix needs to be drained from an access valve on the ammonia tanks and 
the oil needs to be separated from the ammonia. Draining this mixture puts the FM worker in direct contact with the dangerous anhydrous ammonia. In addition, the tanks are located in a noisy compressor room.

Based on the above FM test case, an ASIT can be loaded with the applicable safety information. Figure 50 presents the data load applicable for this FM test case.

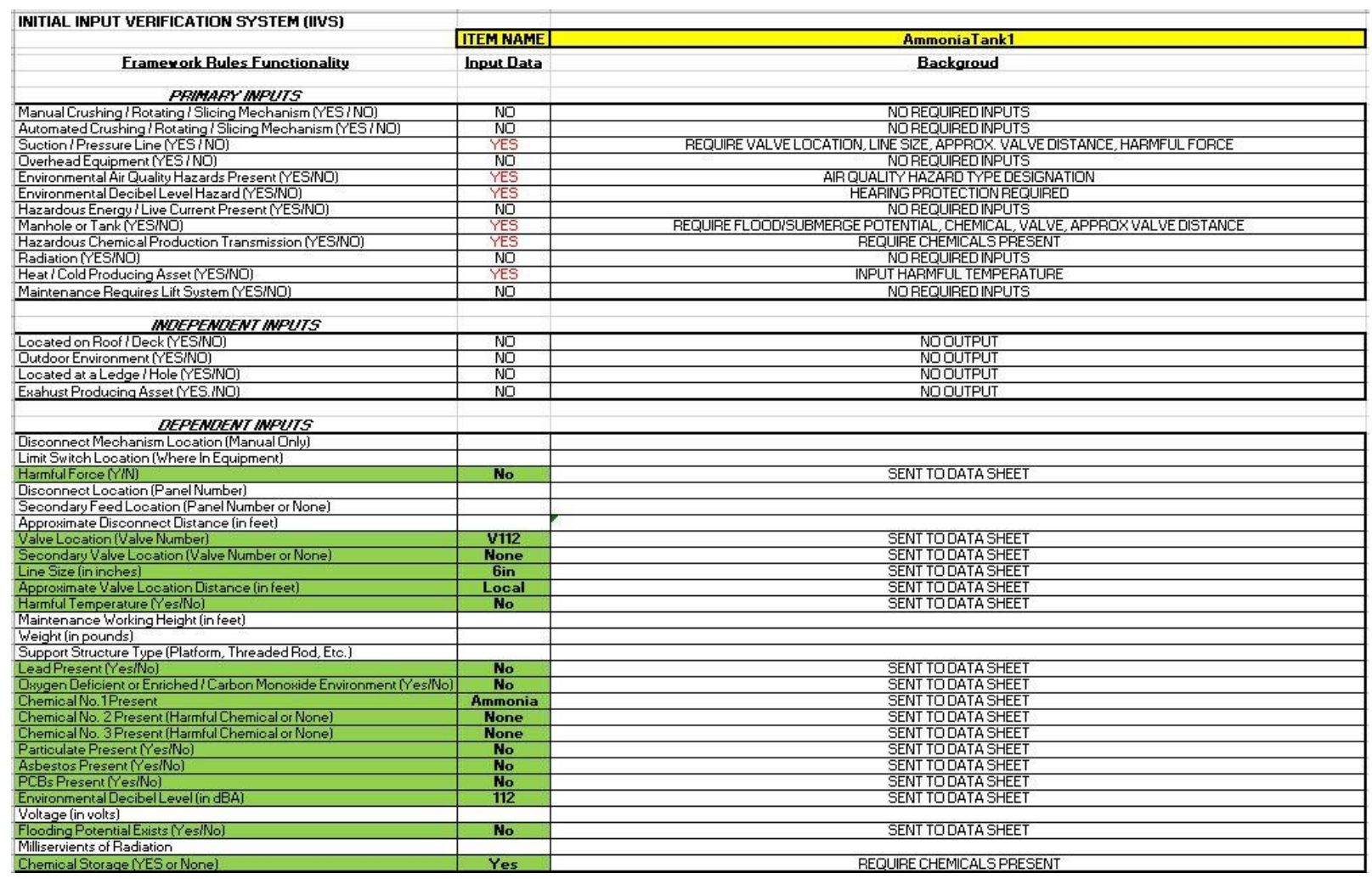

Figure 50: ASIT Data Load for AmmoniaTank1 - FM Test Case No. 4

Table 30 presents the values required for the AmmoniaTank for this particular test case. 
Table 30: Test Case No. 4 Relevant ASIT Data for AmmoniaTank1

\begin{tabular}{|c|c|c|}
\hline Input Type & Inputs & Value \\
\hline Primary & Suction/Pressure Lines & Yes \\
\hline Dependent & Valve Location & V112 \\
\hline Dependent & Line Size & 6in \\
\hline Dependent & Approximate Valve Location Distance & Local \\
\hline Primary & Environmental Air Quality & Yes \\
\hline Dependent & Chemical No. 1 Present & Liquid Anhydrous Ammonia \\
\hline Primary & Environmental Decibel Level Hazard & Yes \\
\hline Dependent & Environmental Decibel Level & $112 \mathrm{dBA}$ \\
\hline Primary & Manhole / Tank / Confined Space & Yes \\
\hline Dependent & Valve Location & V112 \\
\hline Dependent & Approximate Valve Location Distance & Local \\
\hline Dependent & Chemical Storage & Yes \\
\hline Primary & $\begin{array}{l}\text { Hazardous Chemical } \\
\text { Production/Transmission }\end{array}$ & Yes \\
\hline Dependent & Chemical No. 1 Present & Liquid Anhydrous Ammonia \\
\hline Primary & Heat / Cold Producing Asset & Yes \\
\hline Dependent & Harmful Temperature & No \\
\hline
\end{tabular}

Figure 51 outlines the process the DRPS would execute in order to return results to the FM worker prior to executing the FM task. 


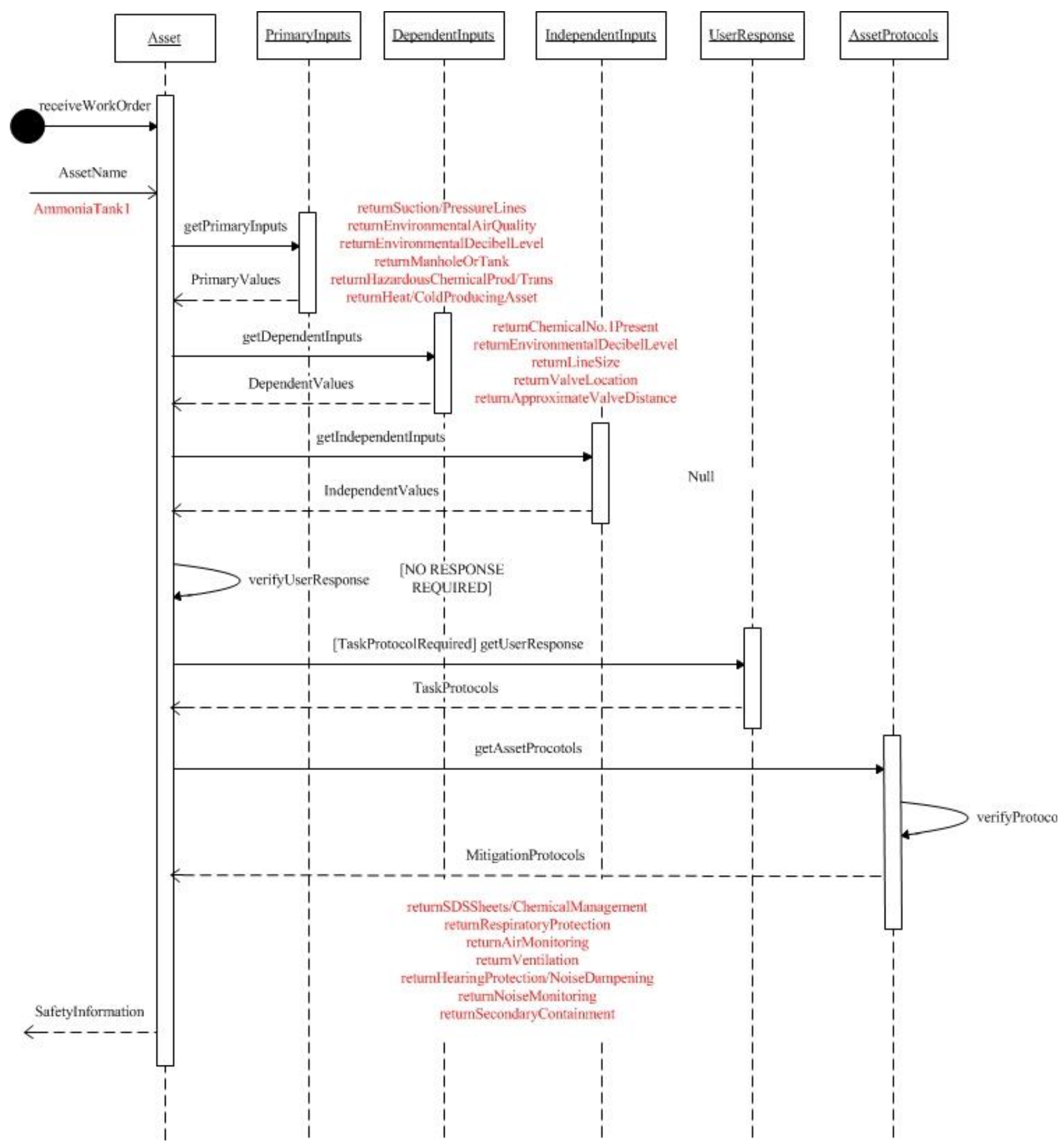

Figure 51: Sequence Diagram for AmmoniaTank1 - FM Test Case No. 4

Upon receiving the work order and launching the DRPS, the system will identify the Asset and execute the getPrimaryInputs, getDependentInputs, and getIndependentInputs command. Executing these commands for the asset requiring maintenance returns the Primaryvalues (Suction/PressureLines, EnvironmentalAirquality, EnvironmentalDecibelLevel, Manhole/Tank/ConfinedSpace, 
DependentValues (ChemicalNo.1Present, EnvironmentalDecibellevel, LineSize, ValveLocation, ApproximateValveDistance). Once the DRPS returns all of the applicable values, the system can process the information and verify if a query should be launched. In this scenario, no query system launch is required and therefore the getUserInput action is bypassed. With all of the inputs identified, the system can evaluate and return the MitigationProtocols for the specific FM task. The protocols being returned in this test case address three hazards:

1. Environmental Air Quality

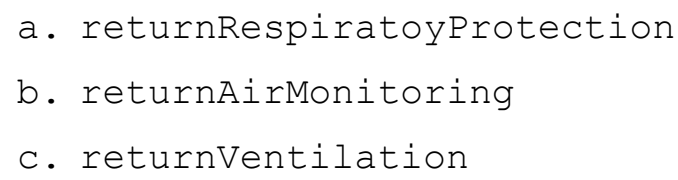

2. Environmental Decibel Level

a. returnHearingProtection/NoiseDampening

b. returnNoiseMonitoring

3. Chemical Production / Transmission
a. returnSDSSheets/ChemicalManagement
b. returnSecondaryContainment

\subsection{DRPS Proof of Concept}

In order to achieve the final validation step set forth by Turkaslan-Bulbul and Akin (2007), the functional usage of the data model or Support, the research graphically presents a conceptual GUI in correlation with the sequence diagram presented in FM Test Case No. 4 - Liquid Anhydrous Ammonia \& Oil Separation. Developing and validating the DRPS conceptual GUI executes the final objectives of this research, as well as completes the final phases of the Six Sigma methodology - Design and Verify. Utilizing the conceptual GUI with the sequence diagrams presents a visual representation of the user interface and the background information being retrieved/returned by the DRPS. These combined graphics serve as a proof of concept for the DRPS.

The basis of the DRPS developed within this research is BIM-based, launched within an appended Autodesk Navisworks model as presented in Chapter 5. In execution, this requires the utilization of the Autodesk Navisworks Software Developer's Kit (SDK) over the Navisworks .NET Application Programming Interface (API). Conceptually, the DRPS is a "Plug-in" to Navisworks. Autodesk Inc. (2016) states, "(A) plug-in allows (the user) to write additional functions that extend the Autodesk Navisworks 
product." For this research, the BIM-based safety framework would utilize Navisworks' data storage capacity and graphics and extend the software by adding the DRPS on top of the existing system. The following section provides the conceptual graphics, built in Java Eclipse Window Builder, in correlation with the sequence diagrams to present a proof of concept to the functional usage of the DRPS. The model utilized within the following section is representative and does not include all of the graphics within a complete compressor package.

\subsubsection{DRPS Proof of Concept Test Case}

The following is a restating of Test Case No. 4 - Liquid Anhydrous Ammonia \& Oil Separation:

A FM Worker at an ice skating rink needs to maintain the ammonia tanks that cool a brine solution required for the sheet of ice. The rink utilizes an indirect brine system in order to facilitate heat exchange. The brine solution runs through the ice floor, absorbing the floor's heat and keeping the ice sheet cool. When the brine solution returns to the compressor system, it is run through coils housed in a tank filled with ammonia in order to draw the heat out of the brine solution, cooling the solution to be sent back to the ice rinkfloor. The ammonia compressor in the system utilizes self-lubrication in order to get oil to the mobile parts of the compressor. Over time, the oil seeps from the compressor crankcase and becomes mixed with the liquid anhydrous ammonia. In order to maintain the thermodynamic efficiency of the ammonia surrounding the brine coils, the oil and ammonia mix needs to be drained from an access valve on the ammonia tanks and the oil needs to be separated from the ammonia. Draining this mixture puts the FM worker in direct contact with the dangerous anhydrous ammonia. In addition, the tanks are located in a noisy compressor room.

The behavior of the DRPS is dictated by the sequence diagram presented in Figure 51. In this section, each process that the GUI initiates will have a correlating figure presenting the associated step in the sequence diagram.

STEP 1 - Upon receipt of the work order, the FM user will launch the DRPS plug-in within the appended Navisworks model (Figure 52).

STEP 2 - Launching the DRPS will initiate the system to request the AssetName as shown in Figure 53.

STEP 3 - Upon selection by the user, the Safety Inputs and Values are presented in the asset properties under the "DRPS" tab. Once the user is satisfied with the asset selection, the "Run" button launches the 
DRPS retrieval process. During this process, the DRPS is actively retrieving the PrimaryInputs, DependentInputs, and Independent Inputs and their associated values (Figure 54).

STEP 4 - Once the system has retrieved all of the inputs and values, the DRPS will call on itself to evaluate the returns in order to verifyUserResponse. If the system identifies a safety input that requires additional user input, the system would getUserResponse. In this test case, there is no TaskProtocolRequired and therefore this step is bypassed, moving directly to AssetProtocols retrieval as shown in Figure 55.

STEP 5 - Upon retrieval of the AssetProtocols, the system will call on itself a final time in order to eliminate protocol redundancies as many of the Safety Inputs have shared MitigationProtocols. The returned MitigationProtocols are presented to the user as SafetyInformation (Figure 56), which is in a format ready for user consumption. 


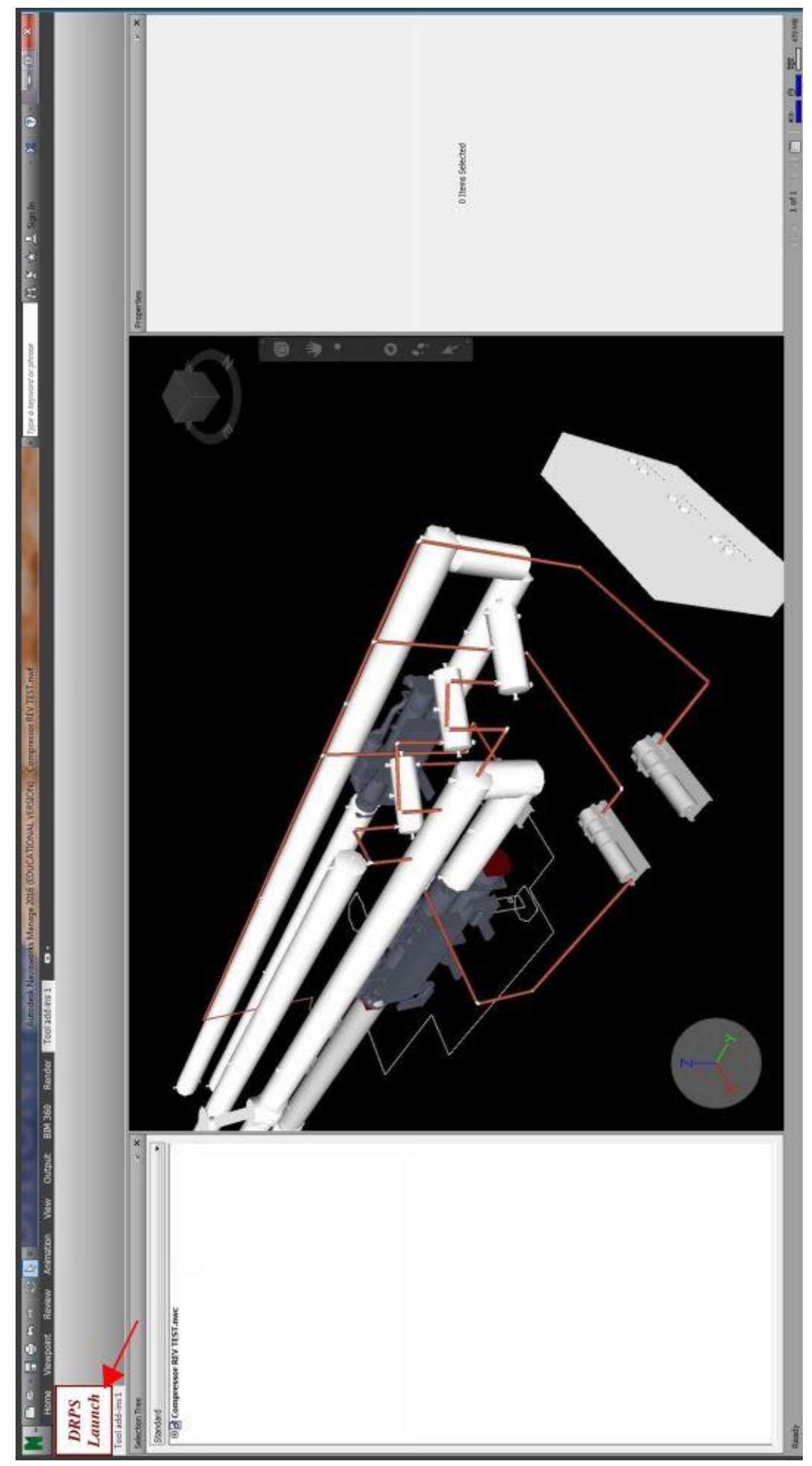

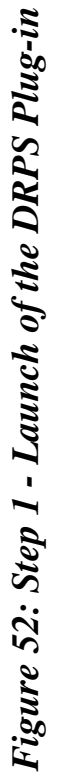




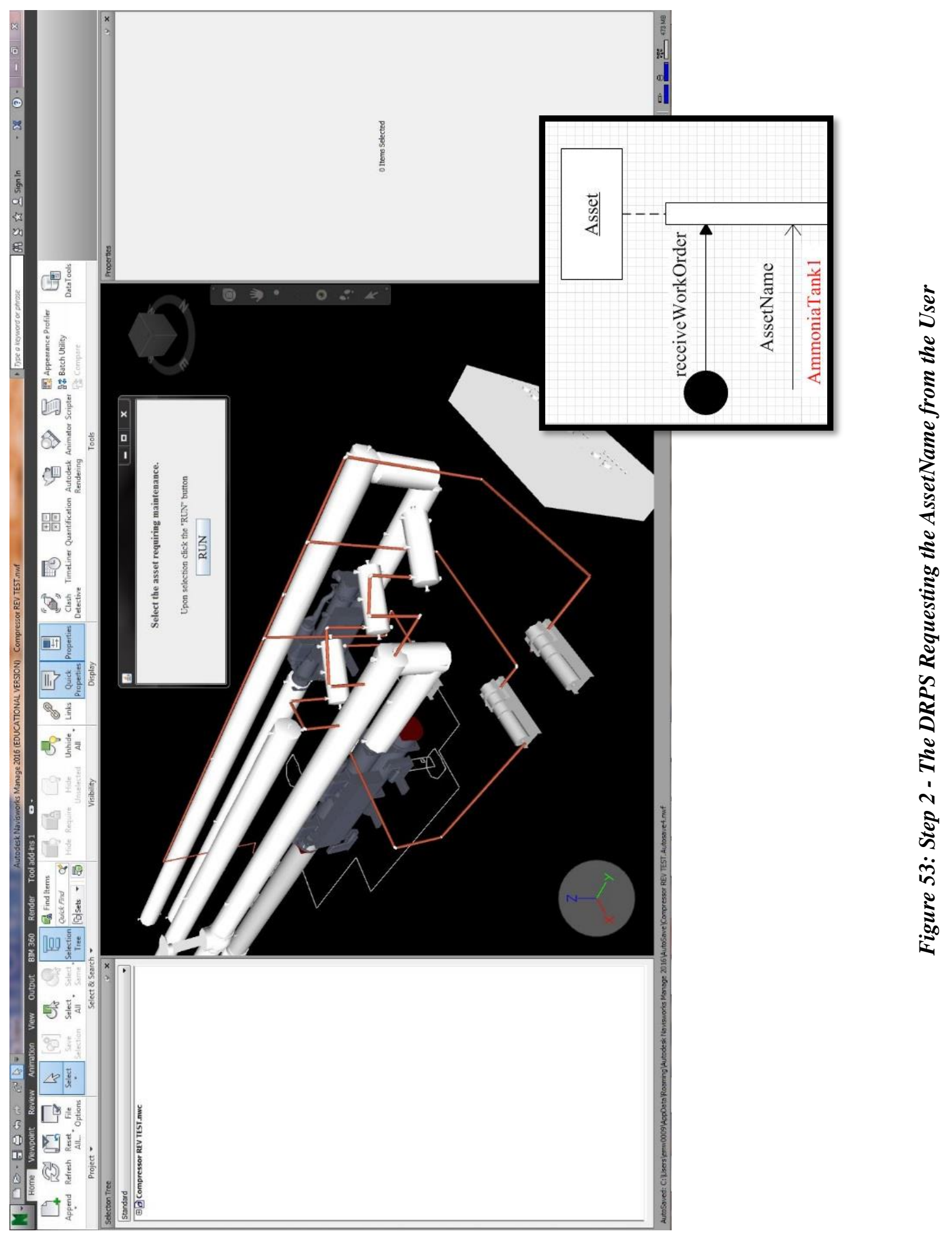




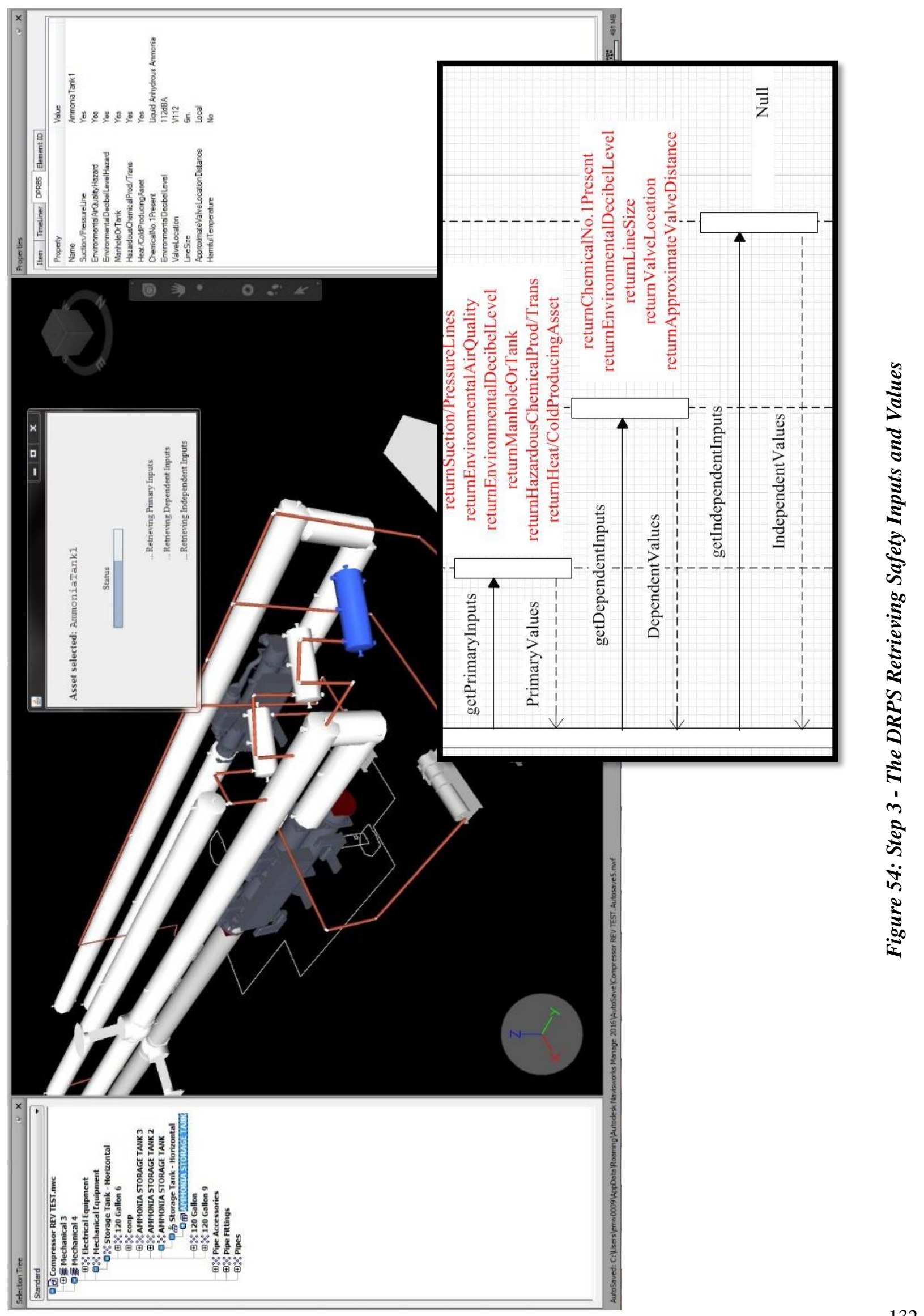




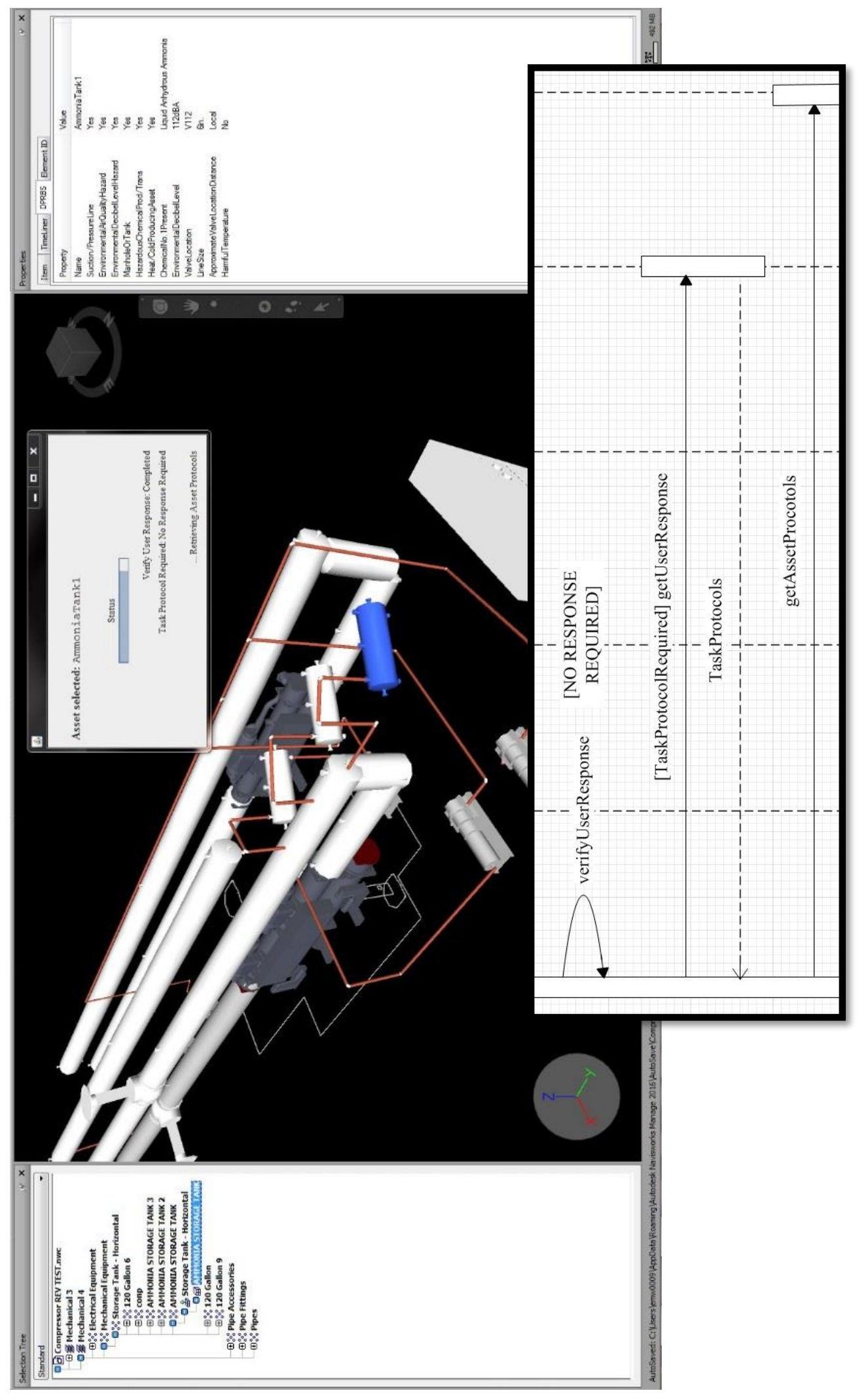

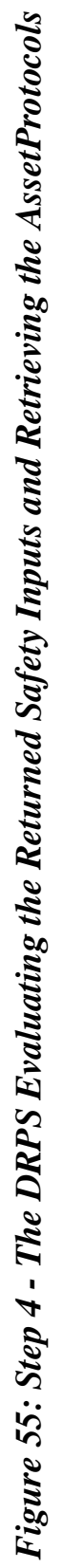




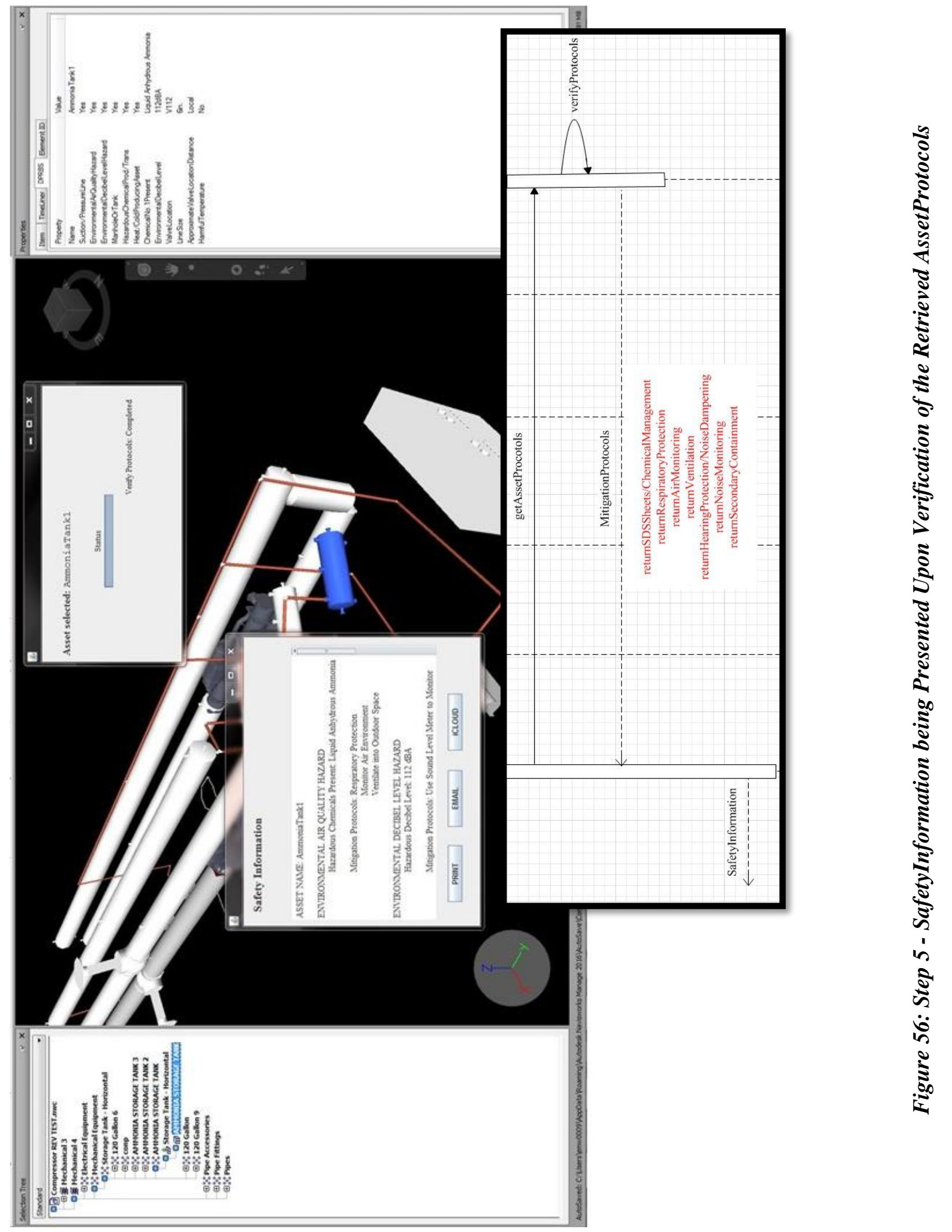




\subsection{Conceptual Walk-through}

The final validation phase of the research includes a conceptual walk-through of the entire proposed framework with industry professionals. The conceptual walk-through is intended to present the framework in order to obtain feedback that could guide the future research and continue to evolve the framework. Utilizing a presentation style format, followed by a conversational exchange, critiques, evaluations, and positives regarding the framework are discussed. For this research, the conceptual walk-through included two safety experts. Table 31 presents the experts included in the walk-through.

Table 31: Conceptual Walk-through Participants

\begin{tabular}{|l|l|l|l|}
\hline Date Interviewed & Pseudonym & Position & Company Type \\
\hline $9 / 26 / 16$ & Ryan & Facilities Safety Inspector & $\begin{array}{l}\text { Large University: East } \\
\text { Region USA }\end{array}$ \\
\hline $9 / 26 / 16$ & Edward & Facilities Safety Coordinator & $\begin{array}{l}\text { Large University: East } \\
\text { Region USA }\end{array}$ \\
\hline
\end{tabular}

The results of this meeting were largely positive towards the concepts and functionality.

- "From a beginning structural aspect, it's pretty good. The concepts are there."

- "I think the functions are good, the yes/no input... it is as simple as it gets."

- "The system is easy enough, there is no rocket science there."

Future research and recommendations for the framework fell within three categories.

\section{Presentation of SafetyInformation}

Identify the best mechanism to deliver the safety information to the user. Currently the system presents text-based safety information, but are different medias more appropriate (e.g. images, videos, etc.)

- "Safety Data Sheets, is that something that you could put in there?"

\section{BIM Model / Technology learning curve}

Maximizing the usability of the system to reduce the learning curve for staff

- "What about those older individuals who are not very computer literate. How do you propose the system to them..." 
Managerial work flow and model updates

Each company using the system will need to identify their particular work flow for safety data loading, backchecking, and model updates

- "It's great stuff and the Return on Investment is there, but getting there is the challenge."

- "Who is maintaining that (the model), you would almost see a person just maintaining the models and information... We are moving to a GIS system, which reads well with BIM, so the need is there."

- [In reference to placing values into the ASIT] "Someone could input the wrong values"

By expanding on the positives from the conceptual walk-through and addressing the items within the future research and recommendations, the framework will continue to evolve. Chapter 8 will address a few of the future research recommendations and summarize the work executed within this research. 


\section{CONCLUSION AND FUTURE RESEARCH}

The inconvenience of having to retrieve uncategorized safety related information from a number of fragmented sources, retards the FM task, requiring time sensitive activities to be rushed, which has been shown to be directly correlated to injuries, illnesses, and fatalities. In an attempt to mitigate facilities management incidents, a BIM-based framework has been developed to deliver comprehensive safety information to FM staff efficiently. Execution of the framework required completion of four objectives:

- Objective No. 1 - Define, categorize, and standardize asset specific safety information applicable to falls, contact with / struck by, and harmful environments \& substances.

- Objective No. 2 - Present a data path through defined transfer mechanisms in order to get safety information, in various formats, from design/construction to FM personnel into a singular repository.

- Objective No. 3 - Develop a data retrieval and processing system (DRPS) and conceptual graphical user interface (GUI), presented through graphics, to represent the interface between FM worker and DRPS.

- Objective No. 4 - Validation

This chapter summarizes how the research achieved these objectives, how this research contributes to the body of knowledge, and avenues for continuation of the research.

\subsection{Execution of Research Objectives}

\subsubsection{Objective No. 1 - Define, Categorize, and Standardize Asset Safety Information}

In order to develop a BIM-based system to process asset specific safety information and present the information to FM staff prior to the execution of an FM task, identifying what information is relevant is the initial step. Through a number of qualitative data collection methods, a comprehensive list of safety inputs (hazards) and protocols (mitigation techniques) regarding falls, contact with / struck by, and harmful environments \& substances were developed. Structuring the static safety information into categories and utilizing the inherent relationships within the data (primary, dependent, and independent), allows for the development of an approach to standardize the safety information based on specific asset groups.

The Asset Safety Identification Tool (ASIT) guides the user through the development of asset specific safety properties. By utilizing known asset information, the formulated spreadsheet can produce a standardized list of safety properties based on the responses to queries within the ASIT. Running facility specific assets through the ASIT provides FM staff with a list of safety properties that require values based 
on the specifics of individual assets. The safety properties can be loaded into a singular BIM-based repository through existing data transfer mechanisms.

\subsubsection{Objective No. 2 - Data Transfer}

Development of a data processing and retrieval system to return asset specific safety information, requires the data be located in a singular data repository. Historically, safety information applicable to the safe maintenance of a facility is fragmented, uncategorized, and turned over to FM staff in a number of different formats (models, docs, pdfs, etc.). The research addresses the fragmentation and categorization of the safety information through the ASIT, but mitigation of the various formats requires the research to identify a data path that centralizes the safety properties in a singular repository. Autodesk Navisworks is used within this research as this BIM-based repository. Navisworks was selected for this research for three reasons:

1. Interoperability - Safety properties loaded into native models can be exported with Autodesk or IFC file extensions, allowing for information to be transferred among software without data loss.

2. Existing Data Transfer Mechanisms - Entities that do not have compatible native models or do not have modeling capabilities will need a secondary mechanism in order to transfer applicable safety properties into the BIM-based Navisworks repository. Using tools built into the Navisworks system, Selection Inspector and DataTools, safety properties can be exported, adjusted in a CSV file, and re-imported back into the BIM model.

3. Navisworks API System - Navisworks allows for "plug-ins" to be written over the Navisworks' .NET Application Programming Interface to extend the base software. Building the Data Processing and Retrieval System (DRPS) with the API negates the need to develop a standalone software with the same interoperability, data transfer/storage capabilities, and graphics that is already built into Navisworks.

Once the safety properties are located within a singular BIM-based repository, the developed Data Processing and Retrieval System (DRPS) can interact with the stored data in order to present asset specific safety information to the FM user.

\subsubsection{Objective No. 3 - DRPS and Conceptual GUI}

Through UML Class and Sequence Diagrams, the structure of the DRPS is presented. The research presents how the DRPS will retrieve and process the safety properties and values stored within the Navisworks repository model using real-life FM test cases. Combining these structural and behavioral diagrams with a conceptual graphical user interface allows for the system functionality to be further presented with graphical context of the user interface. 


\subsubsection{Objective No. 4 - Validation}

Throughout the research, validation of the data and developed processes was of paramount importance. Using data validation interviews, the safety inputs and mitigation techniques obtained during the data collection phase were reviewed, adjusted, and finalized with industry FM safety experts. Using the validated data, the ASIT and the DRPS are developed. As each system was executed, four test cases were used in order to validate that the systems were robust enough to handle diverse FM tasks. As each individual system evolved and the relational connection between the systems was defined (Figure 57), a proof of concept was completed to present the comprehensive functionality of the ASIT, DRPS, and conceptual GUI.

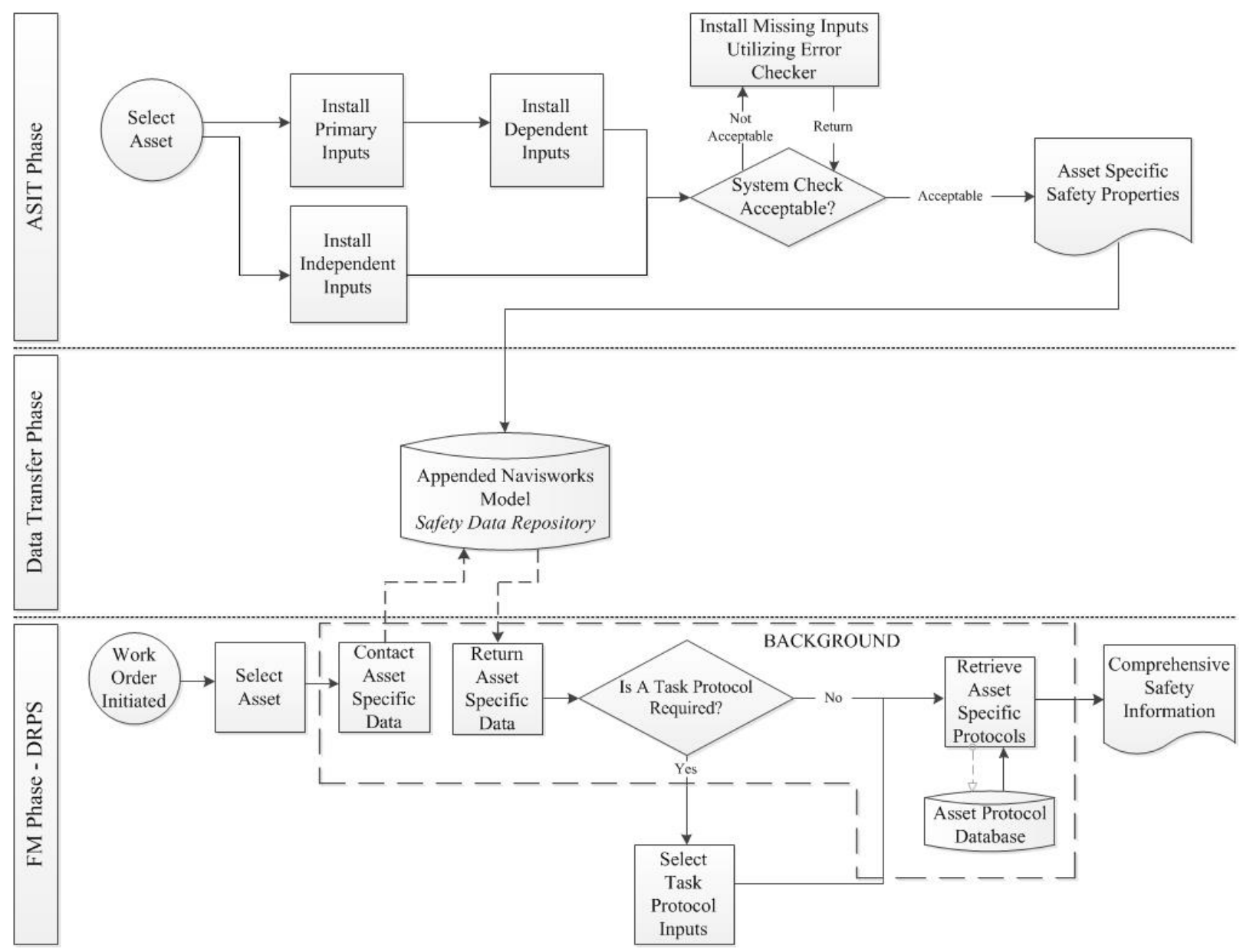

Figure 57: BIM-Based Safety Framework Processes by Phase 


\subsection{Contributions}

The two major contributions of this research is the categorization of safety information and the development of the Asset Safety Identification Tool (ASIT).

The development of categorized safety information regarding falls, contact with / struck by, and harmful environments \& substances is a contribution to the field. Although the FM hazards and associated mitigation techniques were available, the information was fragmented, uncategorized, and left at the discretion of the FM worker of its relevance to asset safety. The benefit of having this safety information available in a comprehensive list focuses the attention of an FM worker to the inherent dangerous of asset safety without the need to reference multiple documents.

The Asset Safety Identification Tool (ASIT) is a formulated spreadsheet, that when executed can produce safety properties for an asset group. The ASIT guides the user through safety property development by requiring responses to queries based on known information about the asset and the research identified safety inputs. This tool will allow for safety property development for any asset group run through the system. The benefit of being able to identify safety properties for an asset group mitigates the fragmentation of safety information and standardizes how the information is structured before being transferred to FM staff.

\subsection{Future Research}

The framework presented in this research is intended to get research defined safety inputs and protocols to FM workers prior to a FM task through the use of a DRPS. The research covers what safety information is applicable to FM workers, how the data is loaded, how the data is transferred to a repository, and how the DRPS interacts with the stored safety information. Creating solutions for these objectives meets the intent of this research; however, a number of research paths can be explored to continuously evolve the framework. The following section presents four new objectives that future research could address.

\subsubsection{Expansion of the DRPS}

The Data Retrieval and Processing System conceptually pulls stores information from the Navisworks repository, processes the data, verifies the need for additional user data, re-processes, and presents the information. In this current format, the system relies heavily on user input and understanding of returned results. Based on the conceptual walk-throughs with the industry experts, minimizing the amount of information processing the user must do and maximizing the functionality of the system would help mitigate the learning curve and organizational implementation. Expanding the DRPS to a rule based system would 
add processing power of the current system and further alleviate the human element to FM hazard mitigation. Examples of expanded functionality could include:

- A rule-based and/or artificial intelligence system that extends the current process by considering known asset information, learning patterns, and eliminating possibilities based on the specific asset

- Integration of the ASIT to the DRPS for a simplified data loading mechanism

- Expand the system to process more than one asset in a single run

\subsubsection{Presentation of SafetyInformation}

As shown in Figure 56, Safety Information is currently presented in text format. Based on the DRPS functionality, upon receipt of the applicable protocols the system will structure the returns in order to present the hazards and mitigation techniques to the user. This SafetyInformation step presents the MitigationProtocols to the user in a format that maximizes information consumption. In order to improve the efficiency and effectiveness of the system, a strong evaluation of how the SafetyInformation is presented to the user is important. A literature review regarding effective mechanisms of information dissemination will need to be executed and a decision addressing the platform of SafetyInformation deliverance (text, audio, video, etc.) should be made. In addition to selection of a media type, addressing the format and structure of how the media is presented is also a potential avenue of future research.

\subsubsection{Prototyping and Usability}

Within the scope of this research, the DRPS and GUI are represented schematically through UML diagrams and conceptual visual aids. In order to test the functionality of the system in full application, a prototype of the initial design will need to be coded and launched over the Navisworks API. Alpha tests would be initiated to verify that the system architecture is commensurate with expectations and revisions to the structural and behavioral design of the system can be made as needed. With a functioning prototype, additional studies could be executed to test the usability and interface of the system. The prototyping phase could also be utilized to launch the system using various hardware (e.g. mobile tablets, smartphones, etc.). Utilizing mobile platforms would expand the usability of the system, as well as prevent the user from having to run the DRPS from a central hub.

\subsubsection{Organizational Implementation}

As with the implementation of any new technology, a workflow of how the systems will be initiated, executed, and updated needs to be developed within an organization. The processes designed within this research, specifically the ASIT and DRPS, will require a market study of how organizations plan to 
approach the usage of the system. For example, adding values to the ASIT is a crucial step in the functionality of the system. Improper loading of data could lead to missing or incorrect safety inputs or mitigation techniques. This would undermine the entire system functionality. A close evaluation of organizational setup, available infrastructure, and best practices could lead to a structured workflow that may include additional contract entities (e.g. utilizing the manufacturer for ASIT data loading). Additionally, the legal aspect of where liability lies in utilizing a system that produces safety information would need to be addressed. Findings of this study could lead to adjustments to the usability, hardware, and functionality of the developed systems. 


\section{REFERENCES}

111th Congress of the United States of America (2010). "Federal Buildings Personnel Training Act of 2012." S3250 United States of America.

AECbytes (2004). "The IFC Building Model: A Look Under the Hood." $<$ http://www.aecbytes.com/feature/2004/IFCmodel.html>. (March 1, 2014).

Alberta Government (2014). "Best Practice - Working Safely in the Heat and Cold." Work Safe Alberta Alberta, Canda.

Alsyouf, I. (2007). "The role of maintenance in improving companies' productivity and profitability." International Journal of Production Economics, 105(1), 70-78.

Anfara, V. A., Brown, K. M., and Mangione, T. L. (2002). "Qualitative Analysis on Stage: Making the Research Process More Public." Educational Researcher, 31(7), 28-38.

Atkin, B., and Brooks, A. (2000). "Total Facilities Management." Blackwell Science, Inc., Malden, MA, 1.

Autodesk Inc. (2016). "Autodesk Developer Network - Autodesk Navisworks." <http://usa.autodesk.com/adsk/servlet/index?siteID=123112\&id=15024694>. (August 17, 2016).

Azhar, S., and Behringer, A. "A BIM-based Approach for Communicating and Implementing a Construction Site Safety Plan." Proc., 49th ASC Annual International Conference Proceedings.

Banawi, A. A. (2013). "Improving Construction Processes By Integrating Lean, Green, and Six-Sigma." University of Pittsburgh, ed.Pittsburgh, PA, 110.

Baralt, M. (2012). "Coding Qualitative Data." Research Methods in Second Language Acquistion: A Practical Guide, A. Macket, and S. M. Gass, eds., John Wiley \& Sons, Ltd., Chichester, UK, 222-244.

Baxter, P., and Jack, S. (2008). "Qualitative Case Study Methodology: Study Designa nd Implementation for Novice Researchers." The Qualitative Report, 13(4), 554-559.

Becerick-Gerber, B., Jazizadeh, F., Li, N., and Calis, G. (2012). "Application Areas and Data Requirements for BIM-Enabled Facilities Management." Journal of Construction Engineering and Management, 138(3), 431-442.

Behm, M. (2005). "Linking construction fatalities to the design for construction safety concept." Safety Science, 43(8), 589-611.

Benjaoran, V., and Bhokha, S. (2010). "An integrated safety management with construction management using 4D CAD model." Safety Science, 48, 395-403.

Blache, K. M. "Reliability \& Maintenance Center (RMC)." Proc., International Maintenance Excellence Conference, 21-24.

Booty, F. (2009). "Facilities Management Handbook." Taylor and Francis, Abingdon, Oxford, 383-384.

Broquetas, M. (2010). "List of BIM Software \& Providers." < http://www.cad-addict.com/2010/03/list-ofbim-software-providers.html>. (February 16, 2014).

Bryde, D., Broquetas, M., and Volm, J. M. (2013). "The project benefits of Building Information Modelling (BIM)." International Journal of Project Mangement, 31(7), 971-980.

buildingSMART International Ltd. (2014). "My Software and IFC." < http://www.buildingsmarttech.org/>. (January 20, 2014).

Bureau of Labor Statistics (2009a). "National Census of Fatal Occupational Injuries in 2008 - SOC 499071." Fatal occupational injuries by selected worker characteristics and selected occupation, All U.S., all ownerships, 2008, US Department of Labor, ed.Washington, DC.

Bureau of Labor Statistics (2009b). "Employer-Reported Workplace Injuries and Illnesses in 2008 - SOC 49-9071." Number of nonfatal occupational injuries and illnesses involving days away from work (1) by selected work and case characteristics and occupation, All U.S., private industry, 2008, United States Department of Labor, ed.Washington, DC.

Bureau of Labor Statistics (2010a). "National Census of Fatal Occupational Injuries in 2009 - SOC 499071." Fatal occupational injuries by selected worker characteristics and selected occupation, All U.S., all ownerships, 2009, US Department of Labor, ed.Washington, DC. 
Bureau of Labor Statistics (2010b). "Employer-Reported Workplace Injuries and Illnesses in 2009 - SOC 49-9071." Number of nonfatal occupational injuries and illnesses involving days away from work (1) by selected work and case characteristics and occupation, All U.S., private industry, 2009, United States Department of Labor, ed.Washington, DC.

Bureau of Labor Statistics (2011a). "National Census of Fatal Occupational Injuries in 2010 - SOC 499071." Fatal occupational injuries by selected worker characteristics and selected occupation, All U.S., all ownerships, 2010, US Department of Labor, ed.Washington, DC.

Bureau of Labor Statistics (2011b). "Employer-Reported Workplace Injuries and Illnesses in 2010 - SOC 49-9071." Number of nonfatal occupational injuries and illnesses involving days away from work (1) by selected work and case characteristics and occupation, All U.S., private industry, 2010, United States Department of Labor, ed.Washington, DC.

Bureau of Labor Statistics (2012a). "National Census of Fatal Occupational Injuries in 2011 - SOC 499071." Fatal occupational injuries by selected worker characteristics and selected occupation, All U.S., all ownerships, 2011, US Department of Labor, ed.Washington, DC.

Bureau of Labor Statistics (2012b). "Employer-Reported Workplace Injuries and Illnesses in 2011 - SOC 49-9071." Number of nonfatal occupational injuries and illnesses involving days away from work (1) by selected work and case characteristics and occupation, All U.S., private industry, 2011, United States Department of Labor, ed.Washington, DC.

Bureau of Labor Statistics (2013a). "National Census of Fatal Occupational Injuries in 2012 - SOC 499071." Fatal occupational injuries by selected worker characteristics and selected occupation, All U.S., all ownerships, 2012, US Department of Labor, ed.Washington, DC.

Bureau of Labor Statistics (2013b). "Employer-Reported Workplace Injuries and Illnesses in 2012 - SOC 49-9071." Number of nonfatal occupational injuries and illnesses involving days away from work (1) by selected work and case characteristics and occupation, All U.S., private industry, 2012, United States Department of Labor, ed.Washington, DC.

Bureau of Labor Statistics (2013c). "Occupational Employment and Wages, May 2012, 49-9071 Maintenance and Repair Workers, General." <http://www.bls.gov/oes/current/oes499071.htm>. (March 6, 2014).

Bureau of Labor Statistics (2014a). "General Maintenance and Repair Workers." <http://www.bls.gov/ooh/installation-maintenance-and-repair/general-maintenance-and-repairworkers.htm\#tab-3>. (February 24, 2014).

Bureau of Labor Statistics (2014b). "Job Outlook - General Maintenance and Repair Workers." <http://www.bls.gov/ooh/installation-maintenance-and-repair/general-maintenance-and-repairworkers.htm\#tab-6>. (March 6, 2014).

Buzan, T., and Buzan, B. (1996). The Mind Map Book: How to Use Radiant Thinking to Mazimize Your Brain's Untapped Potential, Plume, New Yok, NY.

Chandrasekaran, B., Josephson, J. R., and Benjamins, V. R. (1999). "What Are Ontologies and Why Do We Need Them?" IEEE Intelligent Systems, 14(1), 20-26.

City of DuPont (2010). "Safety and Accident Prevention Plan."DuPont, Washington, 106-108.

Cleveland, A. B. "Handover Everywhere." Proc., Associated Schools of Construction 50th Annual Conference.

Coastal Carolina University (2003). "Policies \& Procedures Manual - Facilities Management." Coastal Carolina University, Conway, South Carolina, 35.

Commonwealth of Massachusetts (2014). "Injured Workers' Frequently Asked Questions." <http://www.mass.gov/lwd/workers-compensation/injured-workers/injured-workers-faqs.html>. (April 30, 2014).

Cotts, D. G., Roper, K. O., and Payant, R. P. (2010). Facility Management Handbook (3rd Edition), AMACOM - Book Division of American Management Association, New York, NY.

Creighton University Facilities Management (2010). "Facilities Management Safety Handbook." Creighton University, Omaha, Nebraska, 40. 
Crutchfield, N., and Roughton, J. (2014). "Safety Culture." An Innovative Leadership Approach, Elsevier Inc., Waltham, MA, 23-39.

Department of the Navy (2013). "Fall-Protection Guide for Ashore Facilities."Washington, D.C., 6-8.

Duke Facilities Management (2014). "5 Minute Safety Talks." <https://fmd.duke.edu/safety/safety_plan_program/5\%20Minute\%20Safety\%20Talks.php>. (March 21, 2014).

Duke University (2012). "Hearing Conservation Safety Program." <https://fmd.duke.edu/documents/Safety\%20Documents/FMD\%20Safety\%20Programs/hearing \%20conservation/Hearing\%20Conservation\%20Program\%2014Mar12.pdf>. (October 8, 2015).

East, E. W. (2007). "Construction Operations Building Information Exchange (COBIE) - Requirements Definition and Pilot Implementation Standard." Construction Engineering Resarch Laboratory (CERL), Champaign, IL, 195.

East, E. W., and Brodt, W. (2007). "BIM for Construction Handover." Journal of Building Information Modeling, 28-35.

Eastman, C. M. (1999). Building Product Models: Computer Environments, Supporting Design and Construction, CRC Press, Boca Raton, FL.

Eastman, C. M., Teicholz, P., Scaks, R., and Liston, K. (2008). BIM Handbook: A Guide to Building Information Modeling for Owners, Managers, Designers, Engineers, and Contractors, John Wiley \& Sons, Inc., Hoboken, New Jersey.

EcoDomus Inc. (2016). "EcoDomus FM / BIM Software for Lifecycle Facilities Management." $<$ http://www.ecodomus.com/index.php/ecodomus-fm/>. (March 8, 2016).

Environmental Protection Agency (2013). "Greening EPA Glossary." $<$ http://www.epa.gov/oaintrnt/glossary.htm>.

Environmental Protection Agency (2015). "The Truth about Lead Paint Poisoning." <https://www.epa.gov/sites/production/files/documents/bldg-mgr-brochure.pdf>. (6/14/16, 2016).

Epstein, E. (2012). "Implementing Successful Building Information Modeling." Artech House, Norwood, MA, 1-5.

Facilities and Services (2014). "Facilities \& Services Safety Tool Box Talks." $<$ http://www.fs.illinois.edu/services/safety-and-compliance/news/2013/06/25/facilities-servicessafety-tool-box-talks>. (March 21, 2014).

FacilitiesNet (2014). "FM Quick Reads on Third-Party Service Providers: Considering Outsourcing Facilities Mangement." <http://www.facilitiesnet.com/outsourcing/tip/Considering-OutsourcingFacilities-Management--20285>. (April 3, 2014).

Fallon, K., and Palmer, M. (2006). "Capital Facilities Information Handover Guide, Part 1." National Institute of Standards and Technology.

Federal Aviation Administration (2009). "Safety Management System Components." <https://www.faa.gov/about/initiatives/sms/explained/components/>. (April 2, 2014).

Federal Aviation Administration (2010). "Aviation Safety: Safety Management System (SMS)." <https://www.faa.gov/about/initiatives/sms/>. (April 3, 2014).

Fritz, K. (2008). "Case Study \& Narrative Analysis." <http://ocw.jhsph.edu/courses/qualitativedataanalysis/PDFs/Session4.pdf>. (May 1, 2014).

Gager, A. (2014). "Deferred Maintenance: Turning Crisis Into Strategy." <http://www.facilitiesnet.com/facility_maintenance_decisions_columnists/article/DeferredMaintenance-Turning-Crisis-Into-Strategy--15056? source=part>. (January 10, 2015).

Gallaher, M., O'Conner, A. C., Dettbarn, J. L., and Gliday, L. T. (2004). "Cost Analysis of Inadequate Interoperability in the U.S. Capital Facilities Industry." National Institute of Standards \& Technology, Gaithersburg, MD, 6-15.

Gambatese, J. A., Behm, M., and Hinze, J. W. (2005). "Viability of Designing for Construction Worker Safety." Journal of Construction Engineering and Management, 131(9), 1029-1036.

Gambatese, J. A., Behm, M., and Rajendran, S. (2008). "Design's role in construction accident causality and prevention: Perspectives from an expert panel." Safety Science, 46(4), 675-691. 
Gambatese, J. A., Hinze, J. W., and Haas, C. T. (1997). "Tool To Design For Construction Worker Safety." Journal of Architectural Engineering, 3(1), 32-41.

Global FM (2012). "The Importance of FM." <http://globalfm.org/importance-of-fm>. (March 3, 2014).

Godfrey, S. S., Rothstein, P. R., and Laughery, K. R. "Warnings: Do They Make a Difference?" Proc., Proceedings of the Human Factors and Ergonomics Society Annual Meeting, 669-673.

Goedert, J., and Meadati, P. (2008). "Integrating Construction Process Documentation into Building Information Modeling." Journal of Construction Engineering and Management, 137(7), 509-516.

Gruber, T. R. (1993). "A translation approach to portable ontology specifications." Knowledge Acquisition, 5(2), 199-220.

Guba, E. G., and Lincoln, Y. S. (1982). "Epistemological and Methodological Bases of Naturalistic Inquiry." Educational Communication and Technology, 30(4), 233-252.

Guest, G., MacQueen, K. M., and Namey, E. E. (2012). "Applied Thematic Analysis." Applied Thematic Analysis, Sage Publications, Thousand Oaks, CA, 287.

Guldenmund, F. W. (2000). "The nature of safety culture: a review of theory and research." Safety Science, 34, 215-257.

Hadikusumo, B. H. W., and Rowlinson, S. (2002). "Integration of virtually real construction model and design-for-safety process database." Automation in Construction, 11(5), 501-509.

Hammad, A., Setayeshgar, S., Zhang, C., and Asen, Y. "Automatic Generation of Dynamic Virtual Fences As Part of BIM-Based Prevention Program for Construction Safety." Proc., Proceedings of the 2012 Winter Simulation Conference (WSC).

Harris, B., and Rygielski, L. (2014). "Building Automation: Save Money, Reduce Maintenance \& Improve Facility Management." <http://www.facilitymanagement.com/articles/buildingproducts1-0610.html>. (January 10, 2015).

Hecker, S., Gambatese, J. A., and Weinstein, M. (2005). "Designing for Worker Safety." Professional Safety, 50(9), 32-44.

Her Majesty Stationary Office (1994). "Construction (design and management) regulations, statutory instrument 1994." 3410.

Hinze, J., and Wiegand, F. (1992). "Role of Designers in Construction Worker Safety." Journal of Contruction Engineering and Management, 118(4), 677-684.

Holness, G. V. R. (2006). "Future Direction of the Design and Construction Industry." American Society of Heating, Refrigerating, and Air-Conditioning Engineers Inc. (ASHRAE), 48(8), 38-46.

International Facility Management Association (2013). "What is FM?", <http://www.ifma.org/knowbase/browse/what-is-fm->. (February 24, 2014).

International Nuclear Safety Advisory Group (1992). "Safety Culture." 75-INSAG-4, International Atomic Energy Agency, Vienna, Austria.

Kelly, G., Serginson, M., Lockley, S., Dawood, N., and Kassem, M. "BIM for Facility Management: A Review and a Case Study Investigating the Value and Challenges." Proc., Proceedings of the 13th International Conference on Construction Applications of Virtual Reality.

Kiviniemi, A., and Codinhoto, R. (2014). "Challenges in the Implementation of BIM for FM - Case Manchester Town Hall Complex." 2014 International Conference on Computing in Civil and Building Engineering, American Society of Civil Engineers, Orlando, FL, 665-672.

KMIC Controls Inc. (2015). "Understanding Building Automation and Control Systems." $<$ http://www.kmccontrols.com/products/Understanding_Building_Automation_and_Control_Syst ems.aspx>. (April 21, 2015).

Koziolek, S., and Derlukiewicz, D. (2012). "Method of assessing the quality of the design process of construction equipment with the use of DFSS (design for Six Sigma)." Automation in Construction, 22, 223-232.

$\mathrm{Ku}, \mathrm{K}$., and Mills, T. "Research needs for building information modeling for construction safety." Proc., International Proceedings of Associated Schools of Construction 45th Annual Conference.

Lee, K.-L., and Su, Y. (2013). "Applying Six Sigma to Quality Improvement in Construction." Journal of Management in Engineering, 29(4), 464-470. 
Leighton Contractors (2011). "Building a Safety Culture in FM Operations." 〈http://www.fma.com.au/cms/images/events/2011/NSW_Safety_Presentation_110811.pdf〉. (February 27, 2014).

Leveson, N. (2004). "A New Accident Model for Engineering Safer Systems." Safety Science, 42(4), 237270.

Lin, Y.-C., and Su, Y.-C. (2013). "Developing mobile-and BIM-based intergrated visual facility mantenance management system." The Scientific World Journal, Annual 2013, 1-10.

Logan, R. W. (2005). "Design for Six Sigma with Critical-To-Quality Metrics for Research Investments." U. S. D. o. Energy, ed., Universityof California, Livermore, CA, 12.

Logan, R. W., Nitta, C. K., and Chidester, S. K. (2005). "Design for Six Sigma with Critical-To-Quality Metrics for Research Investments." U.S. Department of Energy, ed., Lawerence Livermore National Laboratory, University of California, 12.

Lucas, J. (2012). "An Integrated BIM Framework to Support Facility Management in Healthcare Environments." Virginia Tech, ed.Blacksburg, VA, 108-110.

Lucas, J., and Thabet, W. (2008). "Implementation and evaluation of a VR task-based training tool for conveyor belt safety training." ITcon, 13(Special Issue), 637-659.

Mahasneh, J. K. (2014). "Decision Aid Framework to Implement Soft Skills in Construction Education." V. Tech, ed.Blacksburg, VA, 58.

Maintenance Assistant Inc. (2014). "Reactive Maintenance." $<$ http://www.maintenanceassistant.com/reactive-maintenance/>. (December 29, 2014).

Meadati, P., and Irizarry, J. "BIM-A Knowledge Repository." Proc., Proceedings of the 46th Annual International Conference of the Associated Schools of Construction.

Melzner, J., Hollermann, S., Kirchner, S., and Bargstadt, H.-J. "Model-Based Construction owrk Analysis Considering Process-Related Hazards." Proc., Proceedings of the 2013 Winter Simulation Conference, IEEE, 3203-3214.

Mento, A. J., Martinelli, P., and Jones, R. M. (1999). "Mind Mapping in Executive Education: Applications and Outcomes." Journal of Management Development, 18(4), 390-416.

Mitchell, J., and Schevers, H. "Building Information Modelling for FM using IFC." Proc., CRC Construction Innovation.

Narayanasamy, G. (2015). "DMADV - Another Six Sigma Methodology." <http://www.whatissixsigma.net/six-sigma-dmadv/>. (May 20, 2015).

National Institute for Occupational Safety and Health (1997). "Maintenance Electrician Dies From Crush Injuries When Caught Between Nip Barrier and Upper Frame of Paper Rewinder - Virginia." Fatality Assessment and Control Evaluation Washington, D.C., 4.

National Institute for Occupational Safety and Health (2014). "Fatality Assessment and Control Evaluation Program." <http://wwwn.cdc.gov/NIOSH-

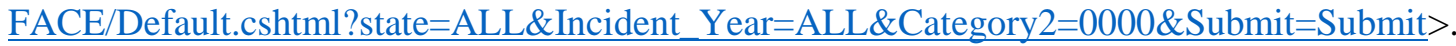
(July 22, 2015).

National Institute of Building Sciences buildingSMART alliance (2014). "National BIM Standard United States Version 2." <http://www.nationalbimstandard.org/>. (March 21, 2014).

Occupational Safety and Health Administration (1998). "Training Requirements in OSHA Standards and Training Guidelines." US Department of Labor, Washington, DC.

Occupational Safety and Health Administration (2004). "Occupational Safety and Health Act of 1970." United States Department of Labor, Washington, DC.

Occupational Safety and Health Administration (2013). "Injury and Illness Prevention Programs."

Occupational Safety and Health Administration (2014). "Q\&A's For Small Business Employers." <https://www.osha.gov/Publications/OSHA3163/osha3163.html>. (March 1, 2014).

Office of Compliance (2010). "Working From Heights: Fall Protection." Fast FactsWashington, D.C., 3. Park, M., Lee, K.-w., Lee, H.-s., Jiayi, P., and Yu, J. (2012). "Ontology-based Construction Knowledge Retrieval System." Korean Society of Civil Engineers Journal of Civil Engineering, 17(7), 16541663. 
Parsanezhad, P., and Dimyadi, J. "Effective Facility Management and Operations Via A BIM-Based Integrated Information System." Proc., CIB Facilities Management Conference 2014, CIB, 442453.

Paslawski, J. (2013). "Hybrid Flexible Approach for Six Sigma Implementation in Constructional SME." Journal of Civil Engineering and Management, 9(5), 718-727.

Patucco, M., Bersano, D., Cigna, C., and Fissore, F. (2010). "Computer image generation for job simulation: An effective approach to occupational Risk Analysis." Safety Science, 40(4), 508-516.

Pavlich, G. (2010). "Paradigmatic Cases." Encyclopedia of Case Study Research, A. J. Mills, G. Durepos, and E. Wiebe, eds., Sage Publications, Inc., Thousand Oaks, CA, 645-648.

Pilone, D., and Pitman, N. (2005). UML 2.0 In A Nutshell, O'Reilly Media, Inc, Sebastopol, CA.

Prevention through Design: Design for Construction Safety (2014). "History of PtD: Origins and Growth." <http://www.designforconstructionsafety.org/history.shtml>. (Januay 18, 2014).

Robson, C. (2011). "Case Studies." Real World Research, John Wiley \& Sons Ltd, West Sussex, United Kingdom, 135-143.

Roelofsen, P. (2002). "The impact of office environments on employee performance: The design of the workplace as a strategy for productivity enhancement." Journal of Facilities Mangement, 1(3), 247-264.

Rossman, G. B., and Rallis, S. F. (2012). Learning in the Field - An Introduction to Qualitative Research, Sage, Thousand Oaks, CA.

Rozenfeld, O., Sacks, R., and Rosenfeld, Y. (2009). "'CHASTE': construction hazard assessment with spatial and temporal exposure." Construction Management and Economics, 27(7), 625-638.

Rumane, A. R. (2013). "Quality Tools for Managing Construction Projects - Industrial Innovation Series." CRC Press, 329-331.

Sabol, L. (2008). "Building Information Modeling \& Facility Management." $<$ http://dcstrategies.net/files/2_sabol_bim_facility.pdf>. (March 3, 2014).

Sapp, D. (2015). "Whole Building Design Guide." <http://www.wbdg.org/om/om.php〉. (April 16, 2015).

Shansolketabi, S. (2013). "Interpreting Chains of Events for Safety Analysis." Norwegian University of Science and Technology, ed., TDT 4900, Trondheim, Norway, iii.

Siemens Technical Education Program (2014). "Basics of Motor Control Centers." <http://www.industry.usa.siemens.com/services/us/en/industry-services/training/self-studycourses/quick-step-courses/Documents/motor control centers.pdf >. (April 30, 2014).

Slaughter, E. S. (2003). "The Link Between Design and Process: Dynamic Process Simulation Models of Construction Activities." 4D CAD and Visualization in Construction: Developments and Applications, Swets \& Zeitlinger, Gainesville, FL, 145-164.

Sokovic, M., Pavletic, D., and Pipan, K. K. (2010). "Quality Improvement Methodologies - PDCA Cycle, RADAR Matrix, DMAIC and DFSS." Journal of Achievements in Materials and Manufacturing Engineering, 43(1), 476-483.

Sorenson, J. N. (2002). "Safety culture: a survey of the state-of-the-art." Reliability Engineering and System Safety, 76, 189-204.

Sulankivi, K., Makela, T., and Kiviniemi, M. "BIM-based site layout and safety planning." Proc., 1st International Conference on Improving Construction and Use through Integrated Design Solutions 2009, CIB IDS.

Sulankivi, K., Zhang, S., Teizer, J., Eastman, C. M., Kiviniemi, M., Romo, I., and Granholm, L. "Utilization of BIM-based Automated Safety Checking in Construction Planning." Proc., CIB World Building Conference.

TabsCAFM (2010). "BMS, CMMS, CAFM, IWMS, EMS...Confused?", <http://www.tabsfm.com/media/blogs/BMS-CMMS-CAFM-IWMS-EMS...confused/>. (September 2, 2015).

Teicholz, P. (2013). "BIM for Facility Managers." John Wiley \& Sons Inc., Hoboken, New Jersey, 1-2.

The Lawrence Berkley National Laboratory (2012). "Facilities Safety Meeting: Hazards of Rushing."Berkley, CA. 
The National Institute for Occupational Safety and Health (NIOSH) (2013). "Prevention Through Design." <http://www.cdc.gov/niosh/topics/ptd/>. (February 25, 2014).

The Pennsylvania State University (2008). "DMADV: An Approach for Developing New Intiatives." Innovation Insights, Office of Planning and Institutional Assessment, State College, PA, 4.

The University of Vermont Risk Management Group and Sarah Forbes Creative (2004). "The University of Vermont Risk Management Handbook." The University of Vermont, Burlington, Vermont, 48.

Tjahjono, B., and Ball, P. (2010). "Six Sigma: A Literature Review." International Journal of Lean Six Sigma, 1(3), 216-233.

Toups, H. J. (2004). "Hazards, Accidents, Process Safety Management \& Process Hazard Analysis." <www.sache.org/links/Pike21Jul2004/PSM\%20and\%20Methods.ppt>. (March 3, 2014).

Tsai, W.-T., Lee, Y.-H., Wiezel, A., Sun, X., and Li, W. (2009). "Ontology-based Service Composition Framework for Syndicating Building Intellegence." IEEE Conference on Commerce and Enterprise Computing, IEEE, Vienna, Austria, 445-452.

Turkaslan-Bulbul, M. T. (2006). "Process and Product Modeling for Computational Support of Building Commissioning." Carnegie Mellon University, ed.Pittsburgh, PA.

Turkaslan-Bulbul, M. T., and Akin, O. (2007). "Evaluation Methods for Building Product Models: Measuing the Performance of Building Commissioning Data Model." Computing in Civil Engineering, 277-280.

U.S. Department of Energy (2012). "Federal Buildings Training Act Cirriculum." General Services Administration, Washington, DC, 3.

United States Department of Labor (2007). "Safeguarding Equipment and Protecting Employees from Amputations." Small Business Safety and Health Management Series, <https://www.osha.gov/Publications/OSHA3170/3170-02R-2007-English.html>. (June 14, 2016).

United States Department of Labor (2011). "The Controls of Hazardous Energy (lockout/tagout)." Regulations (Standards - 29 - CFR), <https://www.osha.gov/pls/oshaweb/owadisp.show_document?p_table=STANDARDS\&p_id=98 04>. (May 12, 2016).

University of Maryland Facilities Management (2012). "Guide to Safety." <https://www.facilities.umd.edu/documents/FM\%20Safety\%20Guide.pdf>. (October 8, 2015).

University of Maryland Facilities Management (2012). "Guide to Safety." University of Maryland, College Park, Maryland, 2.

University of Minnesota Facilities Management (2008). "Confined Space Entry Program." University of Minnesota, Minneapolis, MN, 6.

Venugopal, M., Eastman, C. M., and Teizer, J. (2012). "An Ontological Approach to Building Information Model Exchanges in the Precast/Pre-stressed Concrete Industry." Construction Research Congress 2012, CRC, West Lafayette, IN, 1114-1123.

Vilasini, N., Neitzert, T., and Rotimi, J. (2014). "Developing and evaluating a framework for process improvement in an alliance projct: a New Zealand case study." Construction Management and Economics, 32(6), 625-340.

Visual Paradigm (2016). "Sequence Diagram." < https://www.visualparadigm.com/VPGallery/diagrams/Sequence.html>. (July 21, 2016).

Vredenburgh, A. G. (2002). "Organizational safety: Which management practices are most effective in reducing employee injury rates?" Journal of Safety Research(33), 259-276.

Wetzel, E. M., and Thabet, W. Y. (2015). "The Use of a BIM-Based Framework to Support Safe Facility Management Processes." Automation in Construction, 60(C), 12-24.

Wogalter, M. S., Allison, S. T., and McKenna, N. A. (1989). "Effects of Cost and Social Influence on Warning Compliance." Human Factors: The Journal of the Human Factors and Ergonomics Society, 31(2), 133-140.

Workcover (2001). "Chair Safety in Design Tool." Workcover, New South Wales.

Yan, H., and Damian, P. (2008). "Benefits and barriers of building information modeling." 12th International Conference on Computing in Civil and Building Engineering 2008. 
Yang, Q. Z., and Zhang, Y. (2006). "Semantic Interoperability in Building Design: Methods and Tools." Computer-Aided Design, 38(10), 1099-1112.

Yin, R. K. (2003). Case Study Research: Design and Methods (3rd Edition), Sage Publications, Thousand Oaks, CA.

Zeitlin, L. R. (1994). "Failure to Follow Safety Instructions: Faulty Communication or Risky Decisions?" Human Factors: The Journal of the Human Factors and Ergonomics Society, 36(1), 172-181.

Zhang, S., Boukamp, F., and Teizer, J. (2014). "Onotology-Based Semantic Modeling of Safety Mangement Knowledge." 2014 International Conference on Computing in Civil and Building Engineering, American Society of Civil Engineers, Orlando, FL, 2254-2262.

Zhang, S., Teizer, J., Lee, J.-K., Eastman, C. M., and Venugopal, M. (2013). "Building Information Modeling (BIM) and Safety: Automatic Safety Checking of Construction Models and Schedules." Automation in Construction, 29, 183-195.

Zhou, W., Whyte, J., and Sacks, R. (2012). "Construction safety and digital design: A review." Automation in Construction, 22, 102-111. 


\section{Appendix A: Definition of Terms}

Definition of terms as it pertains to this research.

Building Information Modeling (BIM): A computer-generated model containing precise geometry and relevant data for support during construction, fabrication, procurement and facility lifecycle (Eastman et al. 2008).

BIM FM: The integration of Building Information Modeling during the facility management phase.

Facility Management: A profession that encompasses multiple disciplines such as electrical, mechanical and plumbing to ensure functionality of the built environment by integrating people, place, process, and technology (International Facility Management Association 2013).

Safety Culture: The product of individual and group values, attitudes, and behavior that determine the commitment to health and safety (International Nuclear Safety Advisory Group 1992).

Safety Protocol: Steps to mitigate health hazards in order to safely execute a facility management task. 


\section{Appendix B: FACE Report Data Collection and Analysis Spreadsheets - Falls}

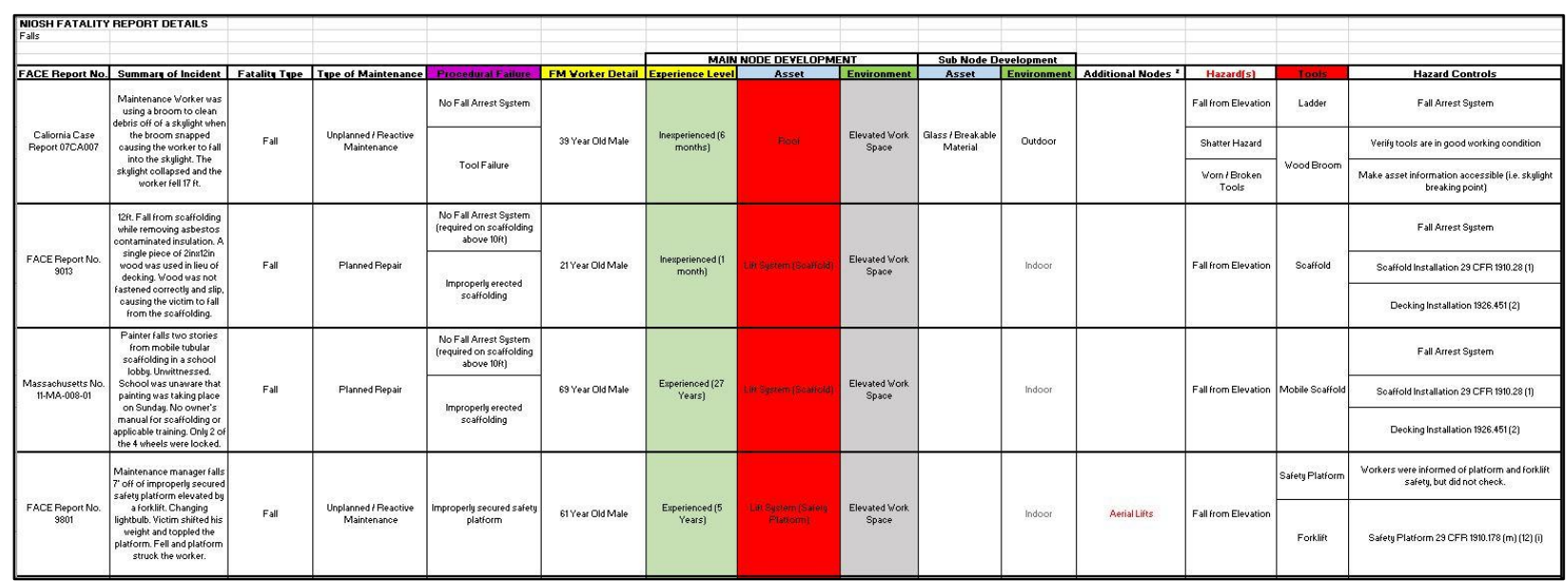

\begin{tabular}{|c|c|c|c|c|c|c|c|c|c|c|c|c|}
\hline $\begin{array}{l}\text { FACE ERpor No. } \\
\text { spot }\end{array}$ & 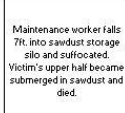 & 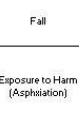 & Planned Maintenanace & 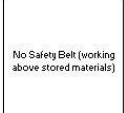 & 52 Yeser oldmale & $\begin{array}{l}\text { Experienced }(8) \\
\text { Yesass }\end{array}$ & 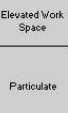 & & Untemon & 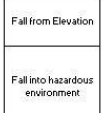 & Fate & 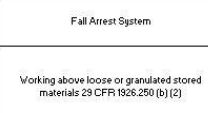 \\
\hline $\begin{array}{l}\text { Michian FACE } \\
\text { Report No. 1.0M0 }\end{array}$ & 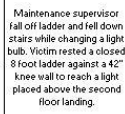 & Fal & 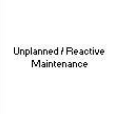 & 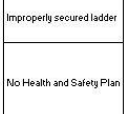 & ${ }_{66}^{6}$ Year old Male & $\begin{array}{l}\text { Experienced(II } \\
\text { Yeass) }\end{array}$ & 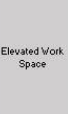 & 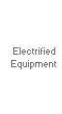 & indoor & Fall fom Elevasion & Lasterer & 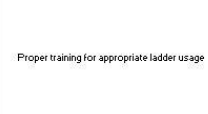 \\
\hline $\begin{array}{l}\text { NYFACE Eeport } \\
\text { OTNYOSOO }\end{array}$ & 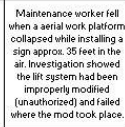 & Fall & Plamered Mimenenance & $\begin{array}{l}\text { Impropoety Modfified } \\
\text { Equverent }\end{array}$ & 54 Year oldmale & 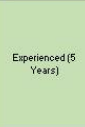 & 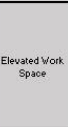 & & Oudoor & Fallirom Elevasion & Aetis Llit & Verity tools ate in \\
\hline 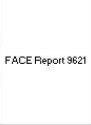 & 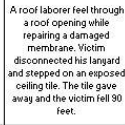 & Fat & 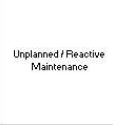 & Diconnected Lanyard & 27 Yese OIIMale & 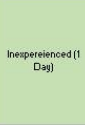 & 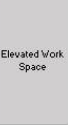 & & Outroor & Fall fom Elevasion & & 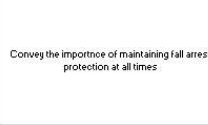 \\
\hline
\end{tabular}

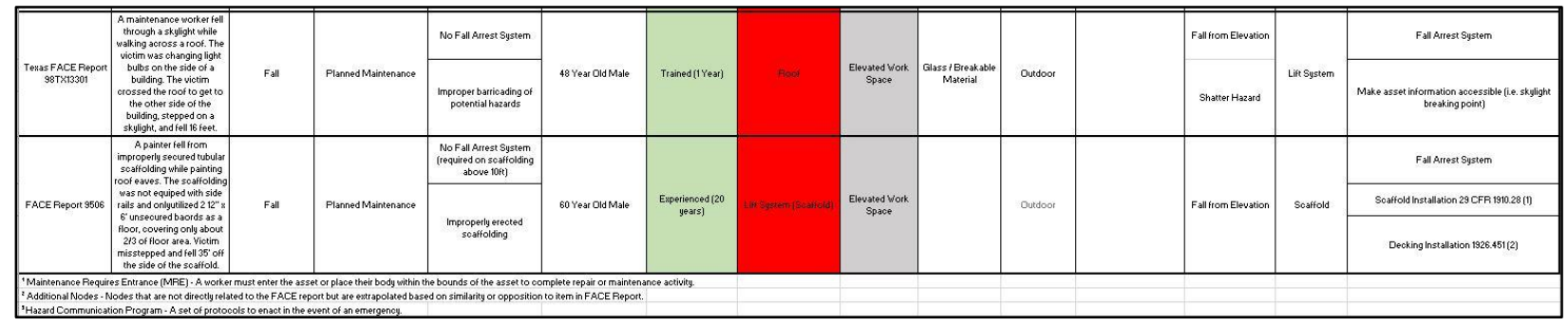


Appendix C: FACE Report Data Collection and Analysis Spreadsheets - Contact With / Struck By
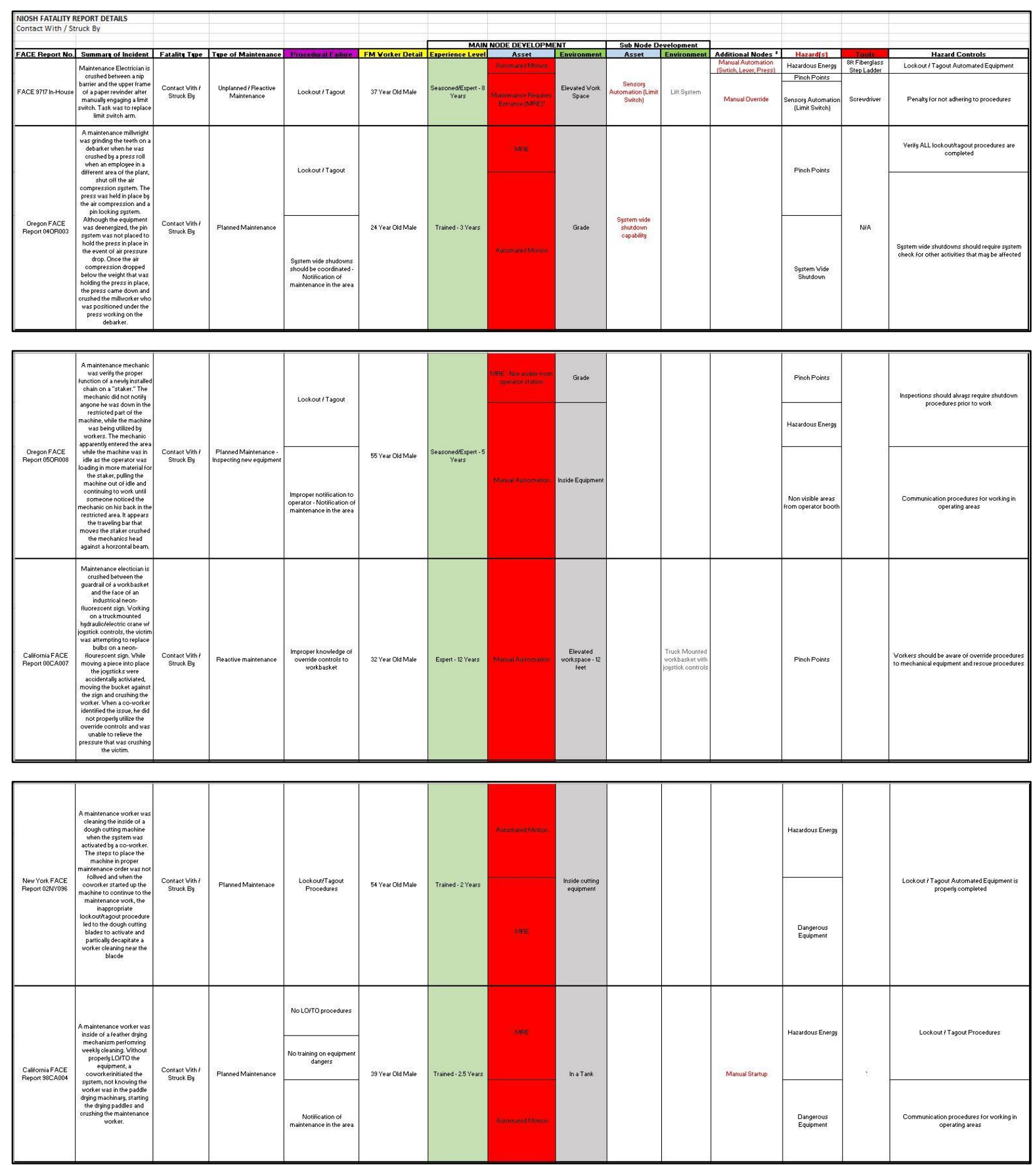

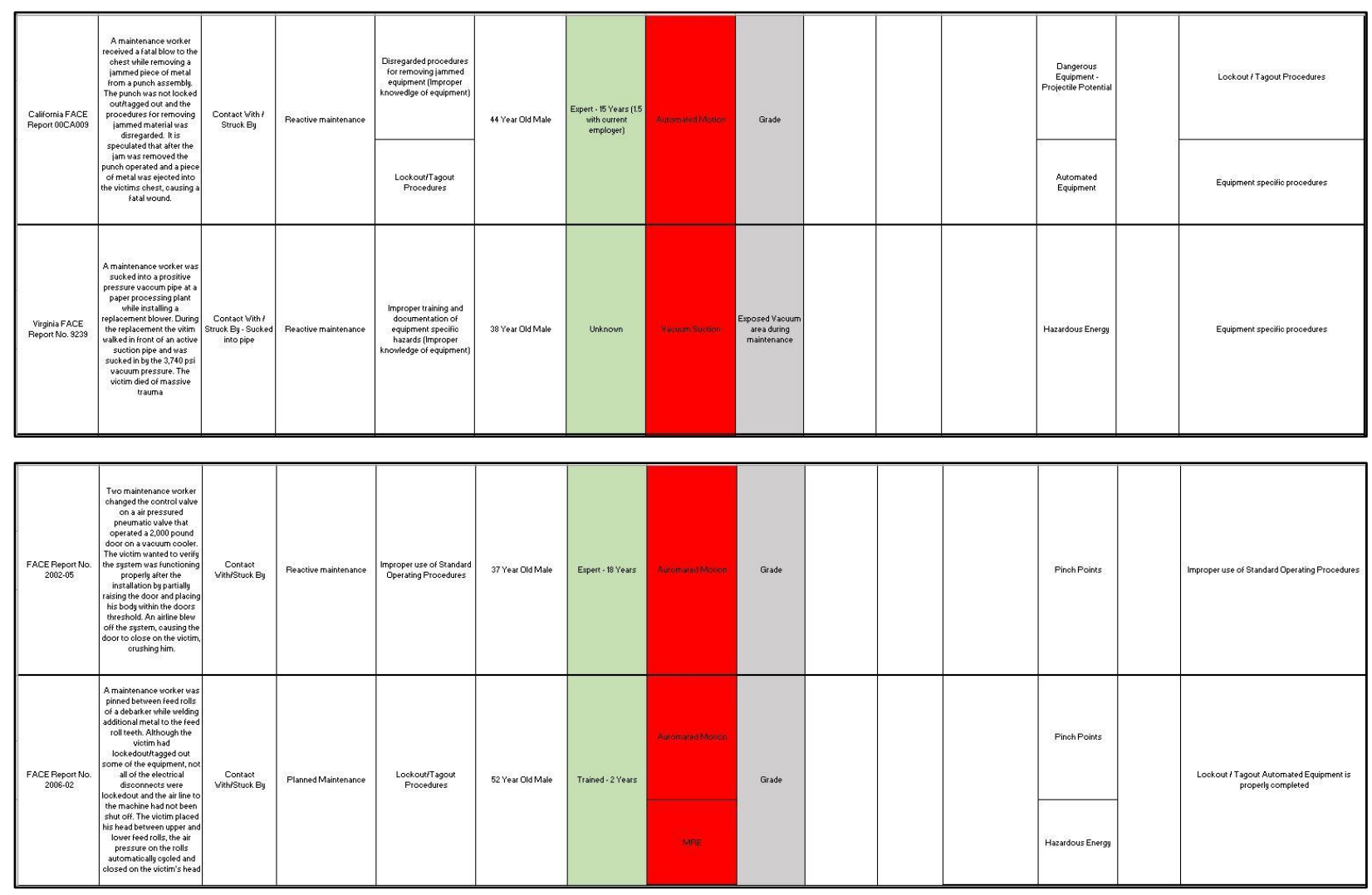
Appendix D: FACE Report Data Collection and Analysis Spreadsheets - Non Elec. H.E\&S.

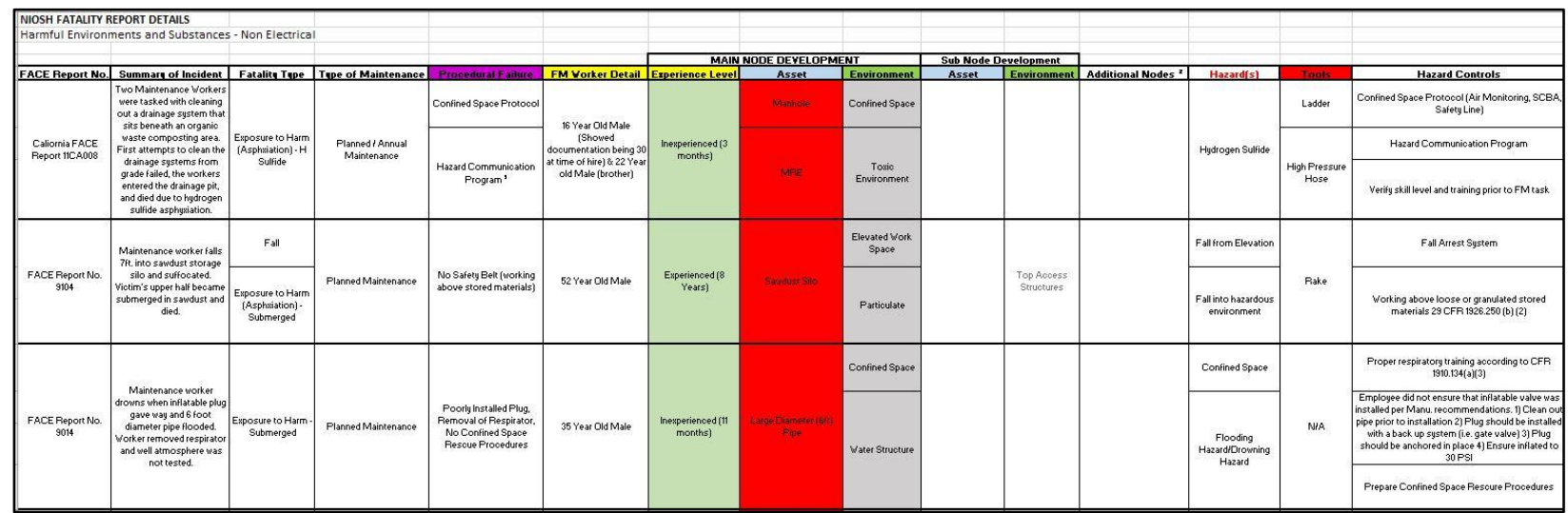

\begin{tabular}{|c|c|c|c|c|c|c|c|c|c|c|}
\hline $\begin{array}{l}\text { FACE Report No. } \\
8928\end{array}$ & 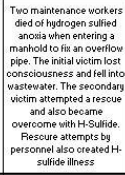 & 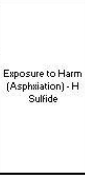 & 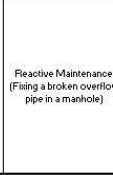 & 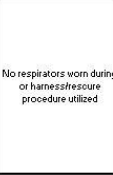 & 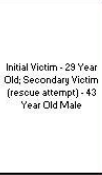 & 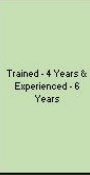 & 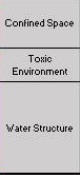 & 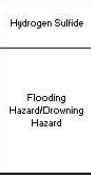 & NAA & 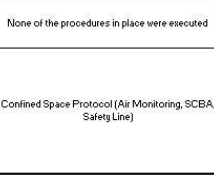 \\
\hline $\begin{array}{l}\text { Colorado FACE } \\
\text { Report } 9100074\end{array}$ & 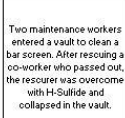 & 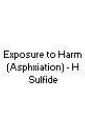 & 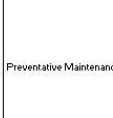 & 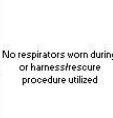 & 49 Yeat Old Male & 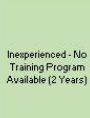 & 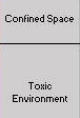 & Hydrogen Surride & NaA & 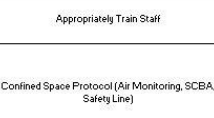 \\
\hline 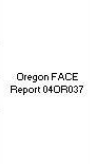 & 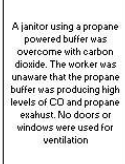 & 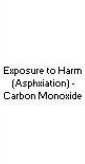 & 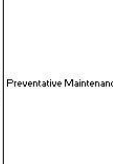 & Improsere ventiation & 50 Yes or old Male & $\begin{array}{l}\text { Noo Tratining }(2.5 \\
\text { weets) }\end{array}$ & 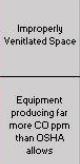 & & 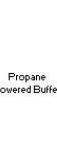 & 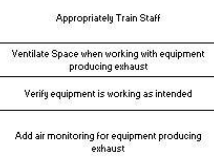 \\
\hline
\end{tabular}

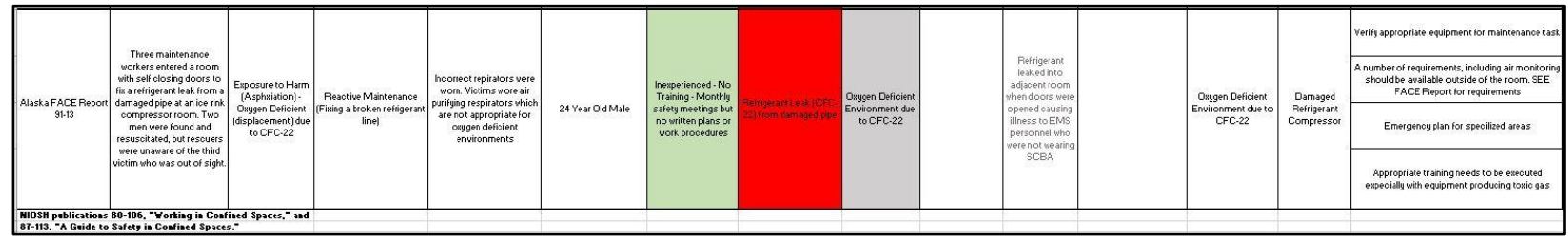


Appendix E: FACE Report Data Collection and Analysis Spreadsheets - Electrical H.E.\&S.
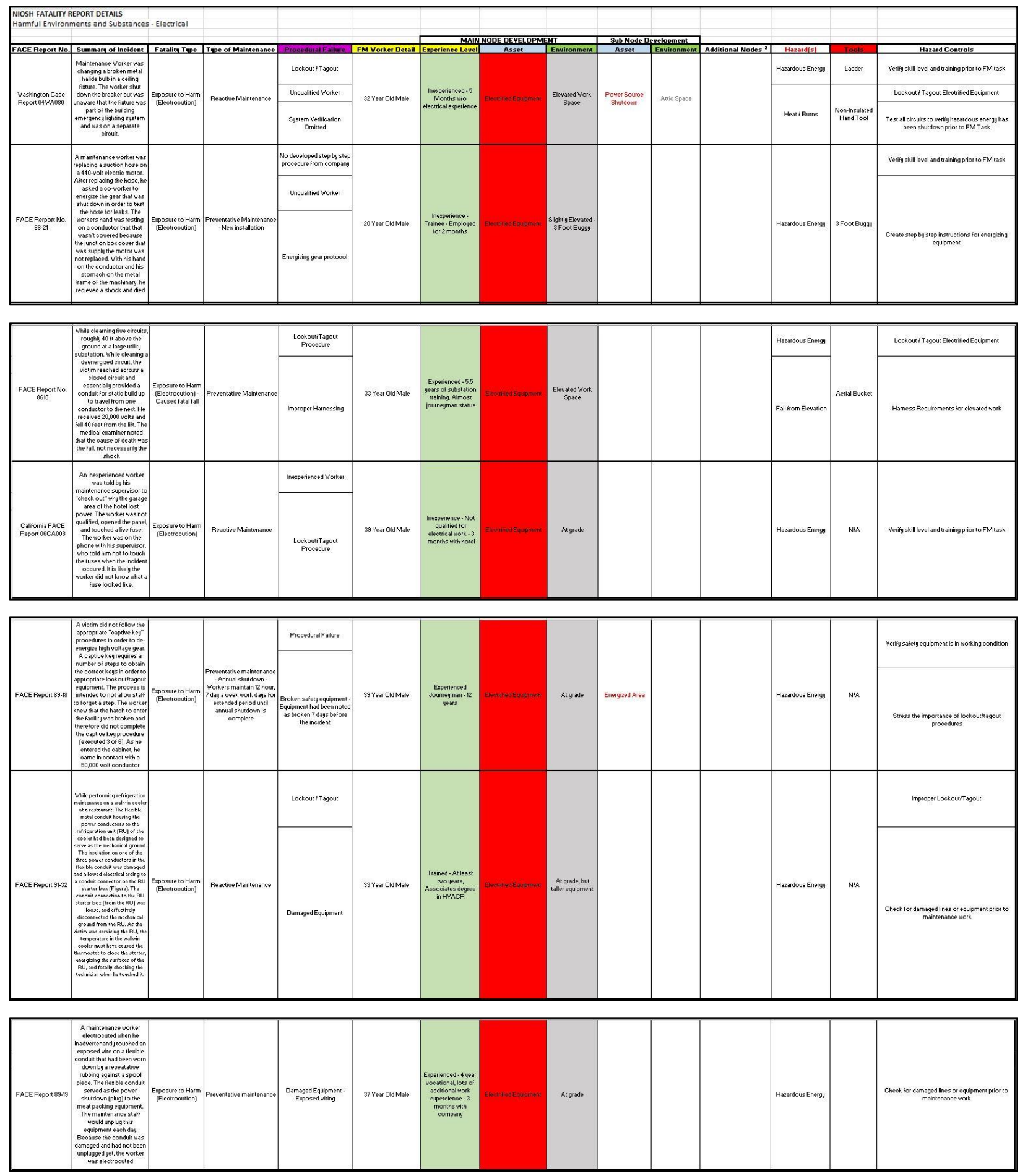


\section{Appendix F: Data Collection Interview Questions}

1. What is your current position?

a. What type of activities do you execute on a daily basis?

b. Do you manage any staff? If so, how many?

2. What type of safety training is done within your company for new and existing employees?
a. How often, how long, what type (videos, teachers, modules, etc.)
b. New vs. existing employees
c. Specific training for certain operations (i.e. Arc flash)
d. OSHA training?

3. Is this method of safety training effective AND is there a method of measuring effectiveness?

4. How is Operations and Maintenance data transferred from construction activities to the facility management staff at the completion of a project?

5. What is the step-by-step process for initiating an FM task at your company?

a. Does the process vary between a preventative or reactive task?

b. What software is utilized in your company for FM tasks? This includes models, work orders, document management, etc.

c. What process is used to identify the equipment that requires maintenance? For example reference the drawings and specs, look at a 3D model, visit the piece or equipment.

d. Are tasks generally executed by a single person or a team?

i. If given to a single person, do safety protocols shift?

6. Where is safety relevant information stored and how is it referenced prior to an FM task?

7. When working at elevation, what are the safety considerations that need to be made?

a. What about in regards to lifting systems, i.e. ladders, scaffolding, buckets, etc.

8. When working in a confined space or toxic environment, what are the safety considerations that need to be made?

a. Is air monitoring utilized?

b. Is there a difference between working in a pipe vs. a manhole vs. a tank?

9. When working with electrified equipment, what are the safety considerations that need to be made?

a. Is there specialized equipment or PPE?

b. What are the training or certification requirements to do electrical work?

c. Is there a difference between working on gear that has high voltage $(600 \mathrm{v}+)$ than low voltage $(<600 \mathrm{~V})$

10. When working with automated equipment, what are the safety considerations that need to be made?

11. How do multiple safety concerns (i.e. working on electrified equipment at elevation) effect the safety preparation of the FM task?

a. Can you think of any other special scenarios that require additional/exceptional safety preparation?

12. In your experience can you provide an example of an accident and why it happened? 
a. How could it have been mitigated?

13. How does age, weather, experience, or any other factors impact the way FM tasks are approached?

14. Provide scenario and ask for input

15. Is there anything you wish you were asked in this interview that was not mentioned or wish to share? 


\section{Appendix G: Data Collection Interview \& Analysis Spreadsheets - Sally}

\begin{tabular}{|c|c|}
\hline & Sally \\
\hline Job Title & Safety Manager for Facilities Management -- Large University, Southeastern Region USA \\
\hline Job Responsibilities & $\begin{array}{l}\text {...schedule safety inspections, program manage meetings, mm, working on safety programs either revising or } \\
\text { completely creating and and being everyone's mothers what I like to call the safety managers wh, we, you } \\
\text { know, I'm out there and I'm really working the guys on projects and and working on the constrvetions sites. } \\
\text { We have small constructions sites that we manage, we have large construction sites that we're program } \\
\text { management but other companies are actually doing the construction... }\end{array}$ \\
\hline Staff Size & Five Hundred Employees (50 in management, 50-75 in management design, remaining maintenance) \\
\hline Training (New Employee) & $\begin{array}{l}\text { A new employee gets a bare minimum depending on their job description. Its emergency management, active } \\
\text { shooter which is very big these days vh, they'll get defensive driving, their fleet management, those are the } \\
\text { basics four and then it expands on from there. Will they be working at heights, then we have to work on the } \\
\text { fall protection, man lift training, will they be going into confined spaces, do they need electrical safety, what, } \\
\text { what do they need for their job and then we we try to pull it out of specials... }\end{array}$ \\
\hline Training (Existing Employee) & $\begin{array}{l}\text { A minimum of one session a month and we require each shop to do a five minute session every week and I } \\
\text { provide all that information for five minute sessions and conduct the majority of the monthly sessions. }\end{array}$ \\
\hline Software & AssetWorks AIM \\
\hline Safety Info in Work Order & $\begin{array}{l}\text {..there now is a a small little note section where they can actually put in safety notes for their employees. } \\
\text { They say oh by the way you need to wear, now don't forget to wear your full face shield and your spagoles } \\
\text { with this one...So their putting in their PPE requirements in as they go, or supposed to be... [Executed by] the } \\
\text { supervisor }\end{array}$ \\
\hline Safety Info Storage & $\begin{array}{l}\text { If someone needed to gain access to a safety protocol or program, we have our safety intranet on Sharepoint. } \\
\text { That has all the current programs that are in place, any documentation they may need, training pieces they } \\
\text { may need, its there. Guidelines, things like that...Every personnel in the facilities has access. }\end{array}$ \\
\hline
\end{tabular}

\begin{tabular}{|c|c|c|}
\hline & \begin{tabular}{|c|} 
Sally \\
\end{tabular} & \begin{tabular}{|r} 
Safety Codes \\
\end{tabular} \\
\hline \multirow{7}{*}{ Falls } & $\begin{array}{l}\text { [MAN POWWRR REQUIREMENT - PROTOCOL] ..if their working at heights, usvally there's more than two } \\
\text { people. There's going to be at least one person on the ground, one person up high, usually two people on the } \\
\text { ground. }\end{array}$ & PROTOCOL: 2 people when working at heights \\
\hline & $\begin{array}{l}\text { [FALLS PROCEDURE]. they're gonna have to look first of all and figure out what piece of equipment do } \\
\text { they need. Do they need a man lift, do they need a nifty lift, do they nees the eighty foot lift or can they do } \\
\text { it all from a ladder. If they can do it from a ladder, which ladder is appropriate. We've had some issves with } \\
\text { this is in the past but they've got better ladders now. And they can actually, some of them actually have cages } \\
\text { on top of them where they can get out and work on the top of the ladder instead of having to pull a lift over. }\end{array}$ & $\begin{array}{l}\text { PROTOCOL: Lift System } \\
\text { ATTRIBUTE: Working Height } \\
\end{array}$ \\
\hline & $\begin{array}{l}\text { [LIFT SYSTEM - BUCKET TRUCK PROTOCOL] . .if they're having to use a lift they know they have to } \\
\text { have fall protection training and they have to have training in hov to operate that lift. Both of which is } \\
\text { about } 12 \text { hours of the training right there. So, they're getting that and they know if they have the training or } \\
\text { they don't have the training. }\end{array}$ & PROTOCOL: Lift System - Bucket Truck \\
\hline & [HARNESS SAFETY] They won't have a safety harness if they don't have the training. & PROTOCOL: Fall Arrest System \\
\hline & $\begin{array}{l}\text { [IIFT SYSTEM - BUCKET TRUCK] ... lot of their work is gonna be done from the bucket truck and they } \\
\text { are always tied off in the bucket truck. }\end{array}$ & PROTOCOL: Lift System - Bucket Truck - Fall Arrest System \\
\hline & $\begin{array}{l}{[\text { [LIFT SYSTEM - BUCKET TRUCK] ...miles per hour is going to based on what the bucket truck can handle }} \\
\text { or the manlift can handle at this point about } 25 \text { miles per hour and we look at the airport numbers, twenty- } \\
\text { five miles per hour is pretty darn high winds and we'll probably shut it down at more like fiftteen because the } \\
\text { guyss are so uncomfortable being up there }\end{array}$ & PROTOCOL: Lift System - Bucket Truck \\
\hline & $\begin{array}{l}\text { [FALL ANCHOR POINTS] ....we don't know where (the fall anchor points) are on all our bvildings, maybe all } \\
\text { our buildings don't have them cavse their all so old. And since we' re a Non-OSHA state we have to fight to } \\
\text { get those things put in place. }\end{array}$ & ATTRIBUTE: Anchor Points (YN) \\
\hline
\end{tabular}




\begin{tabular}{|c|c|c|}
\hline & Sally & Safety Codes \\
\hline \multirow{6}{*}{ Hazardous Environment - Electrical } & $\begin{array}{l}\text { [MAN POWER REQUIREMENT - PROTOCOL] Electricians can trork by themselves depending on what } \\
\text { they are doing, If their doing an assesment, their documenting information, they are making a change in a } \\
\text { light bulb, yes, they can do that by themselves. Most of the time our electricians are in pairs. }\end{array}$ & $\begin{array}{l}\text { PROTOCOL: } 1 \text { person for investigation and simple tasks, } 2 \text { people for more } \\
\text { difficult taaks }\end{array}$ \\
\hline & [LO/TO SAFETY] Same with lockout/tagout, you don't get your lock unless you have the training & PROTOCOL: Lockout/Tagout \\
\hline & $\begin{array}{l}\text { [PROTOCOL - STEP BY STEP] First and foremost, they are going to look at the plans if they don't know } \\
\text { where the line feeds already are and they are going to do every, to make every effort to shut it down and } \\
\text { make sure that it's lockedout tagged out and dead prior to them working on it. }\end{array}$ & $\begin{array}{l}\text { PROTOCOL: Lockout Tagout } \\
\text { ATTRIBUTE: Disconnect Location }\end{array}$ \\
\hline & $\begin{array}{l}\text { [PROTOCOL - HOT WORK] ...they have their processes that are not allowed to work on something um, } \\
\text { that's live unless they have two people, we have all the arc equipment, arc flash equipment that they need to } \\
\text { have. We have several live work programs in place }\end{array}$ & $\begin{array}{l}\text { PROTOCOL: Hotwork - Equipment } \\
\text { PROTOCOL: Hotwork - } 2 \text { people }\end{array}$ \\
\hline & 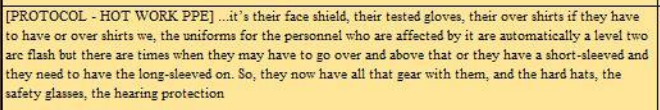 & PROTOCOL: Hotwork - Equipment \\
\hline & $\begin{array}{l}\text { [PROTOCOL - HOT WORK BARRICADING]...it's in an area where they can't just kinda shut the door } \\
\text { then they have to have, you know, the blocking, they have to have someone blocking it off for them and } \\
\text { assuring they have the correct um, distances }\end{array}$ & PROTOCOL: Hotwork - Barricading \\
\hline
\end{tabular}

\begin{tabular}{|c|c|c|}
\hline & Sally & \begin{tabular}{|c} 
Safety Codes \\
\end{tabular} \\
\hline \multirow{5}{*}{ Hazardous Environment - Confined Space Toxic Environment } & $\begin{array}{l}\text { [CONFINED SPACE MONITORNG] ...we just bought brand new monitors, everyone's been trained on how } \\
\text { to use those monitors and we monitor not every } 10 \text { minutes, we monitor continvously, that way we know if } \\
\text { when they re cleaning a storm drain, you know, are they picking up something that, you know, was a pocket } \\
\text { and that we didn't know what was in there. }\end{array}$ & PROTOCOL: Confined Space - Air Monitoring \\
\hline & 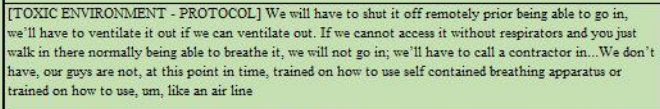 & PROTOCOL: Toxic Environment - Procedure \\
\hline & 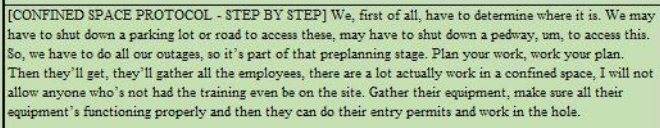 & ATTRIBUTE: Valve Location \\
\hline & [CONFINED SPACE TRAINING] All the person's entering a confined space will be confined space trained & PROTOCOL: Confined Space \\
\hline & Sally & Safety Codes \\
\hline & $\begin{array}{l}{[\text { [AUTOMATED EQUIPMENT - LO/TO] We are constantly doing the lockout/ tagout of HVAC systems for }} \\
\text { that because those are all automated }\end{array}$ & $\begin{array}{l}\text { ATTRIBUTE: Automated Equipment } \\
\text { PROTOCOL: Lockout/Tagout }\end{array}$ \\
\hline Contact With / Struck By & $\begin{array}{l}\text { AUTOMATED EQUIPMENT - SECONDARY LOTO] We also have what I call lockout tagout caution. } \\
\text { Where you are taking a system and putting it down to say twenty percent to change filters out. We have the } \\
\text { tag system for that to notify personnel that may be working behind someone that hey, don't mess with this } \\
\text { system it's down to fifty percent for this reason. Until we have thing we have a yellow tag that we use, very } \\
\text { similar to the lockout tagout but it's not necessarily locked out its just caution tagged out. }\end{array}$ & PROTOCOL: Lockout/Tagout \\
\hline
\end{tabular}

\begin{tabular}{|c|c|c|}
\hline & Sally & Safety Codes \\
\hline \multirow{4}{*}{ Dual Processes } & $\begin{array}{l}{[\text { [CONFINED SPACE AND ELECTRICAL] ...significant amount of underground electrical equipment and }} \\
\text { they are constantly entering manholes valts and having to run a brand ness feed for our stadium or, you } \\
\text { know, to feed that side of the campus to that atvitch and they do have those considerations, there both } \\
\text { considerations. First and foremost, you have to start the confined space process but when you are in a } \\
\text { situation like that }\end{array}$ & FRAMEWORK: Dual Process Concern \\
\hline & $\begin{array}{l}\text { [CONFINED SPACE AND ELECTRICAL - STEP BY STEP] You have to look at the main hazards. You' re } \\
\text { not affected initially by the electrical; you're affected by the confined space. You start that first and you } \\
\text { have that process running and then you go ond do your electrical work. And then you're backing in and } \\
\text { you're backing out. You'rea altways in a confined space and then you have that, to top it off, you could die } \\
\text { when you're in that confined space because of the electrical hazards. }\end{array}$ & FRAMEWORK: Dual Process Concern \\
\hline & $\begin{array}{l}\text { [EXCAVATION AND ELECTRICAL] ...anyone such as our electrical distribution who may have need to } \\
\text { enter an excavation, will have excavation safety, as well as confined space, as well as all the other training } \\
\text { they need to do their iob. Whether it's sp high at heights and they have to vse their man bockets or their lift } \\
\text { truck or it's down on the ground. }\end{array}$ & FRAMEWORK: Dual Process Concern \\
\hline & 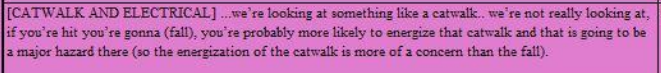 & FRAMEWORK: Dual Process Concern \\
\hline & Sally & Safety Codes \\
\hline \multirow{2}{*}{ Outside Factors to Safety - Environment } & $\begin{array}{l}\text { [HEAT STRESS] The biggest hazard we have here is not necessarily the electrical work because I have } 100 \% \\
\text { trust in my gyys that they can do that right. It's the outside factors like heat stress... Can I make it through } \\
\text { and do this work and still be coherent enough to not pass out from the hast and then we do have an issve with } \\
\text { someone either slumping in of falling out of the bucket. And we've had that }\end{array}$ & PROTOCOL: Outdoor Environment: Heat Stress \\
\hline & $\begin{array}{l}\text { HHEAT STRESS] As for temperature wise it's really more of what can they handle, have they been able to } \\
\text { hande that, are they aclimatized to it, have they been drinking the water, the Gatorade and eating well or } \\
\text { have they not and that's a personal and it's also a kind of a you're your brother's keeper }\end{array}$ & PROTOCOL: Outdoor Environment: Heat Stress \\
\hline
\end{tabular}




\section{Appendix H: Data Collection Interview \& Analysis Spreadsheets - Bill \& Chris}

\begin{tabular}{|c|c|c|}
\hline & Chris & Bill \\
\hline Job Title & Dirsector of Utilities and Energy & Assistant Director of Utilities and Energy \\
\hline Job Responsibilities & 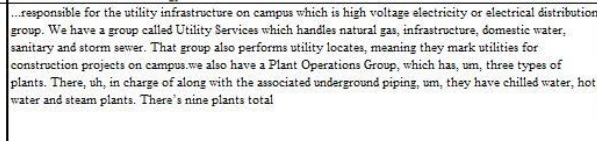 & 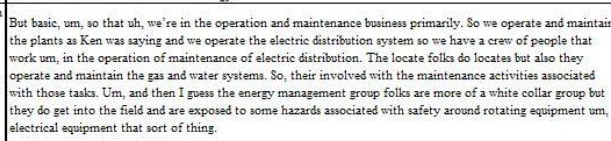 \\
\hline Staff Size & $30+$ & $30+$ \\
\hline Training (New Employee) & 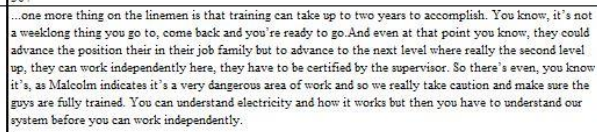 & 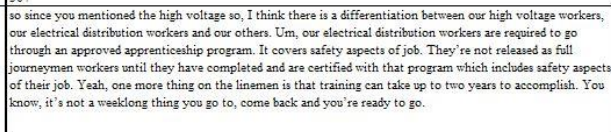 \\
\hline Training (Existing Employee) & & $\begin{array}{l}\text { No sir, we use an outside certified training program but before thay can advance and before they are cut loose so } \\
\text { to speakk, um, doing their job they have to have completed that. Uh, formal training. }\end{array}$ \\
\hline Software & Share Point & \\
\hline $\begin{array}{l}\text { Safety Info in Work Order } \\
\text { Saffety Info Storaze }\end{array}$ & N/A & \\
\hline
\end{tabular}

\begin{tabular}{|c|c|c|}
\hline & Bill and Chris & Safety Codes \\
\hline \multirow{3}{*}{ Falls } & 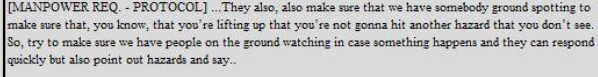 & PROTOCOL: 2 people when working at heights \\
\hline & 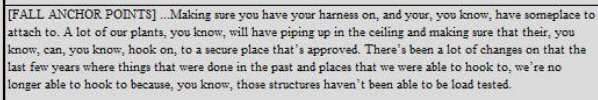 & ATTRIBUTE: Anchor Points \\
\hline & Bill and Chris & Safety Codes \\
\hline \multirow[t]{4}{*}{ Hazardous Environment - Electrical } & 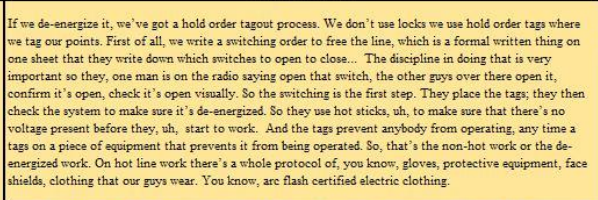 & $\begin{array}{l}\text { PROTCOL: Hold Order Tags } \\
\text { PROTOCOL: : witching Order } \\
\text { ROTOCOL : Hot tsicks } \\
\text { PROTOCOL: PPE }\end{array}$ \\
\hline & $\begin{array}{l}\text { [PPE and MANPOWVRR REQ. If their working energized equipment then they have to have on the proper } \\
\text { level of personal protection per protective equipment. And um, most, not all of those jobs, most of those } \\
\text { jobs are two person jobs. }\end{array}$ & $\begin{array}{l}\text { PROTOCOL: PPE } \\
\text { PROTOCOL: } 2 \text { People for electrical work }\end{array}$ \\
\hline & [HOTSTICKS] Yeah, it's, it's the you always check for voltage before you reach in & PROTOCOL: Hot Sticks \\
\hline & 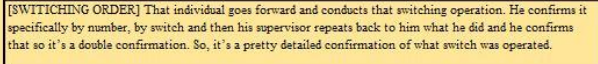 & PROTOCOL: Switching Order \\
\hline
\end{tabular}

\begin{tabular}{|c|c|c|}
\hline & Bill and Chris & $\begin{array}{rr} & \text { Safety Codes } \\
\end{array}$ \\
\hline \multirow{6}{*}{ Hazardous Environment - Confined Space Toxic Environment } & $\begin{array}{l}\text { [CONFINED SPACE - PROTOCOL]].So it's not, it's not a wh. We have some, not like confined space } \\
\text { obviously requires two people but a lot of jobs that are single person jobs. }\end{array}$ & PROTOCOL: 2 People when working in confined space \\
\hline & $\begin{array}{l}\text { [VALVE PROTOCOL] So once they hit the site they see where the leakk is they start figuring out where to } \\
\text { "ut the water, what valves, this valve, this valve, this valve. Shut the valves off. Uh, and then it's a matter of } \\
\text { getting a, you know, system drained down, the laak located. }\end{array}$ & PROTOCOL: Valve Shutdown \\
\hline & 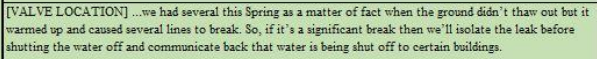 & ATTRIBUTE: Valve Location \\
\hline & 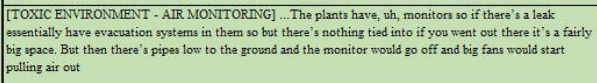 & PROTOCOL: Air Monitoring \\
\hline & 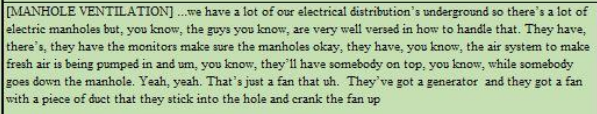 & PROTOCOL: Manhole Ventilation \\
\hline & $\begin{array}{l}{[\text { [MANHOLE PROTOCOLS] ...setting up, you know tha the trolley up where their lifting, and you know, }} \\
\text { putting the mechanism to lift, checking the air in the hole with the meter, putting the air, you know, putting } \\
\text { the ventilation in the hole that sort of thing }\end{array}$ & PROTOCOL: Manhole Work \\
\hline
\end{tabular}




\begin{tabular}{|c|c|c|}
\hline & \begin{tabular}{|c|} 
Bill and Chris \\
\end{tabular} & Safety Codes \\
\hline \multirow{5}{*}{ Contact With / Struck By } & $\begin{array}{l}\text { [PUBLIC SAFETY - BARRICADNG] . But they makes sure that the public is walled off, the students are } \\
\text { walled off there's protection of any sort of interference from cars, of students of pedestrian traffic. }\end{array}$ & PROTOCOL: Barricading \\
\hline & $\begin{array}{l}\text { [AUTOMATED CONTROLS] ...on the plant side, have a lot more of that whare it's a lot more automated } \\
\text { control casse we, we only staff for basically the first thift and our plants run twenty-four seven. }\end{array}$ & ATTRIBUTE: Automated Equipment \\
\hline & 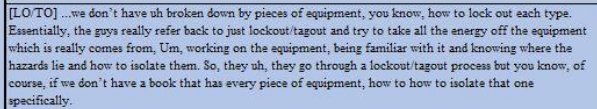 & PROTOCOL: LOTTO \\
\hline & 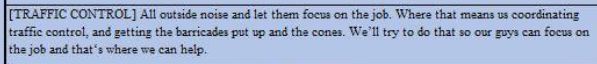 & PROTOCOL: Traffic Control \\
\hline & Bill and Chris & Safety Codes \\
\hline \multirow[t]{2}{*}{ Dual Processes } & \multicolumn{2}{|c|}{ NONE } \\
\hline & \begin{tabular}{|c|} 
Bill and Chris \\
\end{tabular} & Safety Codes \\
\hline \multirow{2}{*}{ Outside Factors to Safety - Environment } & $\begin{array}{l}\text { [MANHOLE VENTIIATION] (When pulling in air to a manhole)...yeah, if you put the fan near the road } \\
\text { and the vehicles are going by they'll svck exhaust }\end{array}$ & PROTOCOL: Special Scenario for roadside manhole ventilation \\
\hline & $\begin{array}{l}\text { FIFAT STRESSI I'll ayy just this time of year another factor which we've, you know, had some isves with } \\
\text { this summer with it being seally hot and humid is the conditions. }\end{array}$ & PROTOCOL: Outdoor Environment: Heat Stress \\
\hline
\end{tabular}




\section{Appendix I: Data Collection Interview \& Analysis Spreadsheets - Joe, Dan, \& Tim}

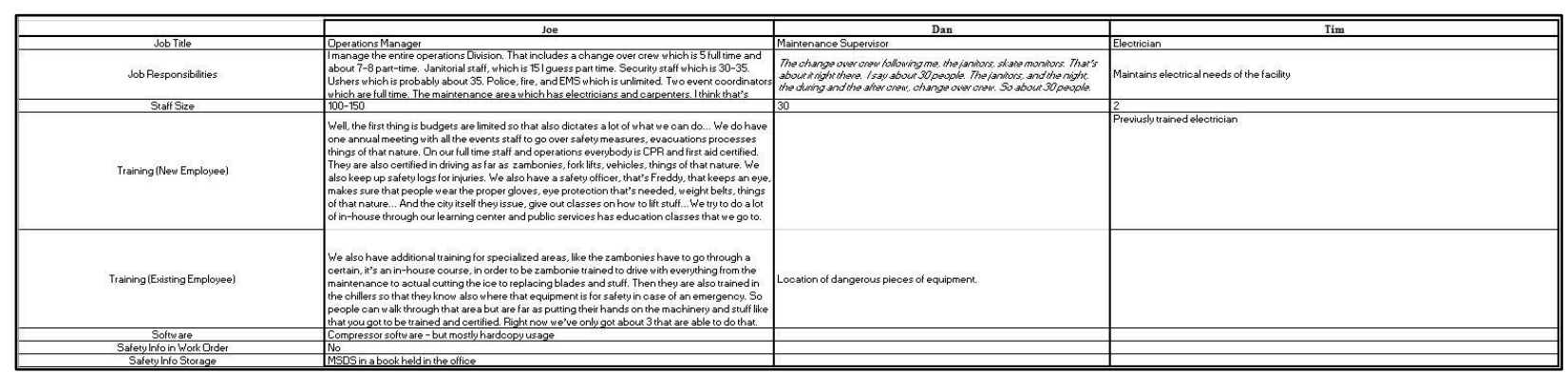

\begin{tabular}{|c|c|c|}
\hline & Joe, Dan, \& Tim & Safety Codes \\
\hline \multirow{3}{*}{ Falls } & $\begin{array}{l}\text { [VALVE AT ELEVATION] ...there's some valves up high. In the chiller room ... There's valves on there that } \\
\text { you have to get up to }\end{array}$ & ATTRIBUTE: Working Height \\
\hline & $\begin{array}{l}\text { [MANLIFT PROTOCOLS] We do have a man lift and Brian's gone over operations with a few gyys but he } \\
\text { mainly ves it... We got a belt you put on and tie off to the bucket... And we also got safety rails and stuff like } \\
\text { that, that lift up and down and lock you in. }\end{array}$ & $\begin{array}{l}\text { PROTOCOL: Lift System } \\
\text { ATTRBBUE: Working Height } \\
\text { PROTOCOL: Fall Arrest System }\end{array}$ \\
\hline & Joe, Dan, \& Tim & Safety Codes \\
\hline \multirow{5}{*}{ Hazardous Environment - Electrical } & $\begin{array}{l}\text { [PPE \& PROTOCOL }- \text { HOT WORK] We try not to work stuff hot, but occasionally they'tl be situations where } \\
\text { you have too. Safety glasses and trying to eliminate the amount of people that are around then there is going to to } \\
\text { be live, exposed electrical dangers... Also note that his boots are different than other folk's boots. Ricky and I } \\
\text { wear steel toe boots but you have a composite boot. }\end{array}$ & $\begin{array}{l}\text { PROTOCOL: Avoid hot work. } \\
\text { PROTOCOL: Proper PPE }\end{array}$ \\
\hline & $\begin{array}{l}\text { [ARC FLASH] Anytime you are dealing with removing a wire or dealing with live, exposed electricity you } \\
\text { present an are flash potential. There's guards inside of the disconnects, inside of the main breakers in there, are } \\
\text { flash guards. }\end{array}$ & PROTOCOL: Arc Flash Potential \\
\hline & $\begin{array}{l}\text { [Hot Stick Usage] A lot of people do. I call them death wands, sometimes they work, sometimes they don't. } \\
\text { They are a good quick test but the only way to be sure is with a meter. }\end{array}$ & PROTOCOL: Hotsticks \\
\hline & $\begin{array}{l}\text { [ELECTRICAL PROTOCOL] people will just go off of somebody else's belief or assumption, that's off, there's } \\
\text { no power there and I go to working on it and got shocked. You can't go off of somebody's telling you that } \\
\text { something's turned off. You should always test it. }\end{array}$ & $\begin{array}{l}\text { PROTOCOL: LO / TO } \\
\text { PROTOCOL: Hotsicks }\end{array}$ \\
\hline & 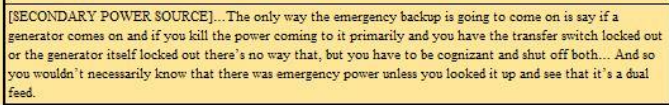 & ATTRIBUTE: Disconnect Locations - Secondary Power Source \\
\hline
\end{tabular}

\begin{tabular}{|c|c|c|}
\hline & Joe, Dan, \& Tim & Safety Codes \\
\hline \multirow{4}{*}{ Hazardous Environment - Confined Space Toxic Environment } & 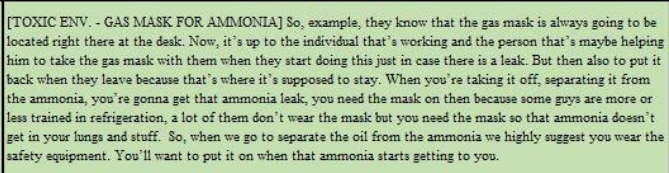 & $\begin{array}{l}\text { PROTOCOL: PPE } \\
\text { ATTRIBUTE: Chemical Environment - Ammonia }\end{array}$ \\
\hline & 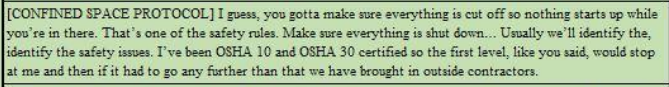 & PROTOCOL: Confined Space - Procedure \\
\hline & $\begin{array}{l}\text { [HEARNVG PROTECTION - COMPRESSOR] I say it's the equivalent of a jackhammer... Yov should certainly } \\
\text { wear ear protection if those compressors are running ... Especially if you have multiple compressors. }\end{array}$ & $\begin{array}{l}\text { ATTRIBUTE: Decibel Level } \\
\text { PROTOCOL: Hearing Protection }\end{array}$ \\
\hline & 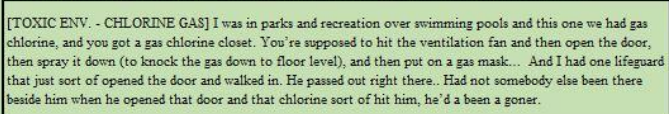 & PROTOCOL: Toxic Environment - Protocol - Chlorine Gas \\
\hline & Joe, Dan, \& Tim & Safety Codes \\
\hline \multirow{6}{*}{ Contact With / Struck By } & $\begin{array}{l}\text { [AUTOMATED EQUIP - DRIVE SHAFTO Right off the bat you never know when the compressors gonna kick } \\
\text { on so if you have any lose clothing near there, that does have a spinning drive shaft... They have partial guards } \\
\text { on them but yes there still exposed, you can get cavght up in them. And it's between where the impellers are the } \\
\text { motors. }\end{array}$ & $\begin{array}{l}\text { ATTRIBUTE: Automated Equipment } \\
\text { PROTOCOL: Lockout Tagout } \\
\text { PROTOCOL: Proper Attire }\end{array}$ \\
\hline & $\begin{array}{l}\text { [AUTOMATED EQUIP - PRESSURIZED LINE] Well, I mean, the pumps that run the brine through the } \\
\text { system they'll kick on. If you're in the middale of all that trying to change a valve or whatever... shut the pumps } \\
\text { off. You can do that from the computer. }\end{array}$ & PROTOCOL: Valve Shutoff \\
\hline & $\begin{array}{l}\text { [AUOMATED EQUIP] Now we do have avtomated motors on, in the other room I showed you, where the } \\
\text { coolers for the HVAC's are and they'll come on automatically. }\end{array}$ & $\begin{array}{l}\text { ATTRIBUTE: Automated Equipment } \\
\text { PROTOCOL: Lockout Tagout }\end{array}$ \\
\hline & 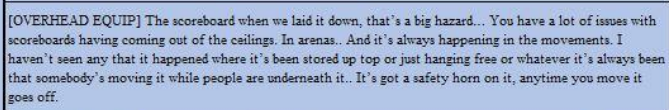 & ATTRIBUTE: Overhead Equipment \\
\hline & 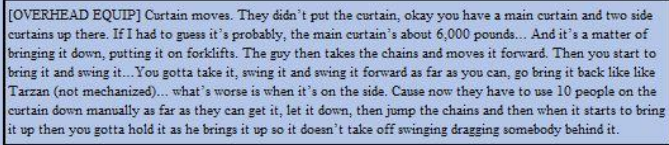 & ATTRIBUTE: Overhead Equipment \\
\hline & $\begin{array}{l}\text { [PRESSURIZED LINE] ...they were digging, putting in that water treatment thing, Either somebody didin't mark } \\
\text { it right or the guy that was doing the digging or whatever, didn't follow his protocols or whatever bot when he } \\
\text { cut that line out, I mean, that water about killed him. }\end{array}$ & ATTRIBUTE: Pressurized Line \\
\hline
\end{tabular}




\begin{tabular}{|c|c|c|}
\hline & Joe, Dan, \& Tim & Safety Codes \\
\hline Dual Processes & 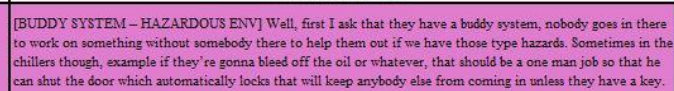 & ROTOCOL: Situational Buddy System \\
\hline & Joe, Dan, \& Tim & \begin{tabular}{|c} 
Safety Codes \\
\end{tabular} \\
\hline Outside Factors to Safety - Environment & 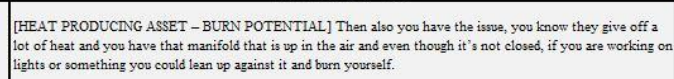 & ATTRIBUTE: Heat Producing Asset \\
\hline
\end{tabular}




\section{Appendix J: Safety Literature Data Collection and Analysis Spreadsheets}

\begin{tabular}{|c|c|c|c|c|c|c|}
\hline \multirow{2}{*}{\multicolumn{7}{|c|}{$\begin{array}{l}\text { Safety Literature Matrix } \\
\text { Safety Schema Development }\end{array}$}} \\
\hline & & & & & & \\
\hline Hazard Type & Source & Hazard & Hazard Definition & Safety Input & Safety Protocol & Protocol Requiremets / Minimum Testing \\
\hline \multirow{6}{*}{$\begin{array}{l}\text { Harmful Environments \& } \\
\text { Substanaces }\end{array}$} & \multirow{6}{*}{$\begin{array}{l}\text { University of Minnesota } \\
\text { Facilities Management }\end{array}$} & \multirow{6}{*}{$\begin{array}{l}\text { Hazardous Atmosphere - } \\
\text { Confined Space }\end{array}$} & \multirow{6}{*}{$\begin{array}{c}\text { An atmosphere presenting a threat of death, } \\
\text { acute injury, illness or disablement due to the } \\
\text { presence of flammable, explosive, toxic, oxygen } \\
\text { deficiency or enrichment, or otherwise injurious } \\
\text { substances }\end{array}$} & Manhole / Tank & Permit Required Before Entry & Obtain permit via organizational standards \\
\hline & & & & $\begin{array}{l}\text { Environmental Air } \\
\text { Quality }\end{array}$ & \multirow{5}{*}{ Atmospheric Testing } & $\begin{array}{l}\text { Flammable gas or vapor concentrations greater than } \\
10 \text { percent of its lower explosive limit ( }>10 \% \text { LEL). }\end{array}$ \\
\hline & & & & \multirow{2}{*}{$\begin{array}{l}\text { Oxygen Deficient/ } \\
\text { Carbon Monoxide } \\
\text { Environment }\end{array}$} & & $\begin{array}{l}\text { Combustible particulate concentrations greater than } \\
10 \text { percent of the minimum explosive concentration } \\
\text { of the particulate ( }>10 \% \mathrm{MEC}) \text {. }\end{array}$ \\
\hline & & & & & & $\begin{array}{l}\text { Atmospheric oxygen concentration either below } \\
19.5 \text { percent or above } 23.5 \text { percent }\end{array}$ \\
\hline & & & & \multirow{2}{*}{ Hazardous Chemicals } & & $\begin{array}{l}\text { Toxic, corrosive or asphyxiate substance } \\
\text { concentrations above Permissible Exposure Levels } \\
\text { and or Threshold Limit Valves ( PEL and or TLV). }\end{array}$ \\
\hline & & & & & & $\begin{array}{l}\text { Any substance that is present at concentrations } \\
\text { greater that the value established as Immediately } \\
\text { Dangerous to Life and Health }(>\mathbb{D L} \text { L). }\end{array}$ \\
\hline \multirow[t]{2}{*}{$\begin{array}{l}\text { Harmful Environments \& } \\
\text { Substanaces }\end{array}$} & \multirow[t]{2}{*}{$\begin{array}{c}\text { Duke University Facilities } \\
\text { Management }\end{array}$} & \multirow[t]{2}{*}{ Hearing Loss } & \multirow{2}{*}{$\begin{array}{c}\text { Workers exposed to } 85 \text { dBA or above time- } \\
\text { weighted average for an } 8 \text { hour period or } 82 \mathrm{dBA} \\
\text { for a } 12 \text { hour time-weighted average, masured on } \\
\text { the A-scale, slow response, or equivalently, a dose } \\
\text { of } 50 \%\end{array}$} & \multirow[t]{2}{*}{$\begin{array}{l}\text { Environmental } \\
\text { Decibel Level }\end{array}$} & Noise Dosimeter & $\begin{array}{l}\text { An instrucment that integrates a function of sound } \\
\text { pressure over a period in such a manner that it } \\
\text { directly indicates a noise dose }\end{array}$ \\
\hline & & & & & Sound Level Meter & An instrument for the measurement of sound level \\
\hline
\end{tabular}

\begin{tabular}{|c|c|c|c|c|c|c|}
\hline \multirow[t]{2}{*}{ Falls } & \multirow[t]{2}{*}{$\begin{array}{l}\text { Creighton Facilitity } \\
\text { Management }\end{array}$} & \multirow[t]{2}{*}{ Fall } & \multirow{2}{*}{$\begin{array}{l}\text { Employees working near unprotected edges or } \\
\text { holes, including skylights, which are six feet } \\
\text { above a lower level, will be protected from falling } \\
\text { by the use of guard rails, positioning devices, a } \\
\text { warning line system, safety monitoring system or } \\
\text { personal fall arrest system. }\end{array}$} & \multirow[t]{2}{*}{ Working Height } & Fall Arrest System & $\begin{array}{l}\text { Fall arrest systems will consist of a harness, self. } \\
\text { retracting lanyard with a deceleration device and } \\
\text { anchor point. Short rope lanyards may be vtilized in } \\
\text { the buckets of man lifts. }\end{array}$ \\
\hline & & & & & Ladder Safety & $\begin{array}{l}\text { Choose the right ladder style (i.e, step or extension), } \\
\text { size and duty rating for the job. Use fiberglass ladders } \\
\text { if there is even a remote possibility of working near } \\
\text { electricity. }\end{array}$ \\
\hline \multirow{3}{*}{$\begin{array}{l}\text { Harmful Environments \& } \\
\text { Substanaces }\end{array}$} & \multirow{3}{*}{$\begin{array}{l}\text { Creighton Facilitity } \\
\text { Management }\end{array}$} & \multirow{3}{*}{ Asbestos } & \multirow{3}{*}{ Airborne carcinogen present in materials } & Asbestos Poetential & Asbestos Management & $\begin{array}{l}\text { Facilities Management employees are prohibited } \\
\text { from performing any work that will intentionally } \\
\text { disturb asbestos containing materials. }\end{array}$ \\
\hline & & & & \multirow[b]{2}{*}{$\begin{array}{l}\text { Environmental Air } \\
\text { Quality }\end{array}$} & \multirow[b]{2}{*}{ Breathing Apparatus } & $\begin{array}{l}\text { Facilities Management employees are prohibited } \\
\text { from performing work in any areas that will, or may, } \\
\text { expose them to airborne asbestos where abatement is } \\
\text { in progress. }\end{array}$ \\
\hline & & & & & & $\begin{array}{l}\text { Facilities Management employees are permitted to } \\
\text { work in areas shere asbestos containing materials } \\
\text { exist as long as the materials are safe from becoming } \\
\text { airborne and providing the employee has the proper } \\
\text { PPE and training. }\end{array}$ \\
\hline
\end{tabular}

\begin{tabular}{|c|c|c|c|c|c|c|}
\hline \multirow{3}{*}{$\begin{array}{l}\text { Harmful Environments \& } \\
\text { Substanaces AND Contact With } \\
\text { /Struck By }\end{array}$} & \multirow{3}{*}{$\begin{array}{l}\text { Creighton Facilitity } \\
\text { Management }\end{array}$} & \multirow{3}{*}{ Hazardous Energy } & \multirow{3}{*}{$\begin{array}{l}\text { All energy sources that might cause unexpected } \\
\text { movement, personal injury or property damage. } \\
\text { This includes electrical, mechanical, hydraulic, } \\
\text { thermal, pneumatic, compressed gas energy, } \\
\text { potential energy from suspended or overhead } \\
\text { objects and compressed springs. }\end{array}$} & $\begin{array}{l}\text { Avtomated } \\
\text { Equipment }\end{array}$ & Lockout / Tagout & $\begin{array}{l}\text { A tagout system will always be used in addition to the } \\
\text { lockout system. Tagout devices must indicate the } \\
\text { reason for the lockout; how that person may be } \\
\text { reached; the identity of the person who applied the } \\
\text { device; and the date and time the tag was placed. }\end{array}$ \\
\hline & & & & Hazardous Energy & Permits & De-Engergize and Re-energize Protocols \\
\hline & & & & \begin{tabular}{|} 
Electrical Disconnect \\
Locations
\end{tabular} & Lockout / Tagout & Same as above \\
\hline \multirow[t]{2}{*}{ Falls } & \multirow[t]{2}{*}{ Office of Compliance } & \multirow[t]{2}{*}{ Fall } & \multirow{2}{*}{$\begin{array}{c}\text { Falls from heights involving scaffolding, rooftops } \\
\text { and ledges }\end{array}$} & Roof / Deck & Fall Arrest System & $\begin{array}{l}\text { Wear a full body harness when required or when } \\
\text { working from elevations such as rooftops or elevated } \\
\text { platforms greater than four feet. }\end{array}$ \\
\hline & & & & Ledge / Hole & Barricading & $\begin{array}{l}\text { If working in a high traffic area, isolate the work } \\
\text { area to prevent potential falling objects from } \\
\text { striking people below. }\end{array}$ \\
\hline
\end{tabular}




\begin{tabular}{|c|c|c|c|c|c|c|}
\hline \multirow{3}{*}{$\begin{array}{l}\text { Harmful Environments \& } \\
\text { Substances }\end{array}$} & \multirow{3}{*}{$\begin{array}{l}\text { Government of Alberta } \\
\text { Canada }\end{array}$} & \multirow{3}{*}{ Environment Temperature } & \multirow{3}{*}{$\begin{array}{c}\text { Employees working in hot and cold outdoor } \\
\text { environments }\end{array}$} & \multirow{3}{*}{$\begin{array}{l}\text { Outdoor } \\
\text { Environment }\end{array}$} & \multirow{3}{*}{ Weather Considerations } & $\begin{array}{l}\text { Serious haelth isves can happen when you are } \\
\text { exposed to extremes of heat or cold. }\end{array}$ \\
\hline & & & & & & $\begin{array}{l}\text { Working in heat can affect the body with: Mental } \\
\text { Changes - increased irritation, mood changes, } \\
\text { deppression, aggression, and anger. Physical Response } \\
\text { - increased heart rate, increased sweating, muscle } \\
\text { cramps, changes in breathing patterns, dizziness, } \\
\text { faintness, or "prickly heat" (heat rash). }\end{array}$ \\
\hline & & & & & & 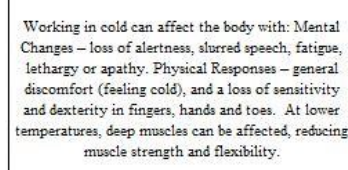 \\
\hline \multirow[t]{3}{*}{ Contact With / Struck By } & \multirow[t]{3}{*}{ USDL } & \multirow[t]{3}{*}{ Equipment Motion } & \multirow[t]{3}{*}{ 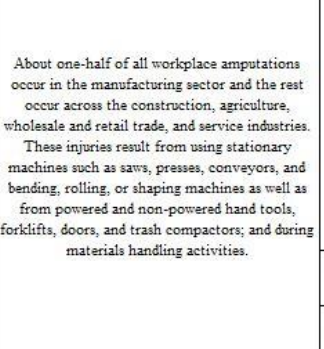 } & $\begin{array}{l}\text { Automated } \\
\text { Equipment }\end{array}$ & \multirow[t]{3}{*}{ Lockoout / Tagout } & \multirow[t]{3}{*}{ 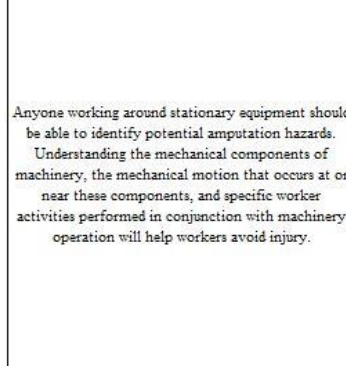 } \\
\hline & & & & Manual Equipment & & \\
\hline & & & & Hazardous Energy & & \\
\hline
\end{tabular}

\begin{tabular}{|c|c|c|c|c|c|c|}
\hline \multirow{2}{*}{$\begin{array}{l}\text { Harmful Environments \& } \\
\text { Substances }\end{array}$} & \multirow{2}{*}{ EPA } & \multirow{2}{*}{ Lead Paint } & \multirow{2}{*}{$\begin{array}{l}\text { Builiding of assets built prior to } 1978 \text { may have } \\
\text { lead based paint }\end{array}$} & \multirow{2}{*}{ Lead Paint Potential } & Breathing Apparatus & $\begin{array}{l}\text { It's not just lead paint chips that poison. } \\
\text { Contamination can be cavsed by only a little bit of } \\
\text { lead dust that is easily absorbod by anyone who } \\
\text { inhales or ingests it. }\end{array}$ \\
\hline & & & & & Lead Management & $\begin{array}{l}\text { Federal taw requires that if you or someone on your } \\
\text { staff is performing the work your firm must be Lead- } \\
\text { Safe Certifited and your staff trained in lead-safe work } \\
\text { practices }\end{array}$ \\
\hline $\begin{array}{l}\text { Harmful Environments \& } \\
\text { Substances }\end{array}$ & USDL & Hazardous Energy & 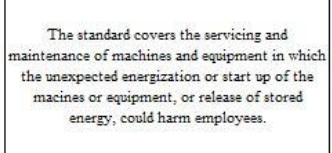 & Hazardous Energy & Lockout / Tagout & 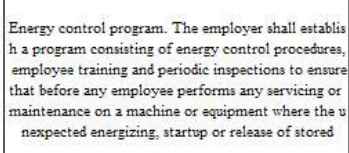 \\
\hline Falls & Department of the Navy & Fall & 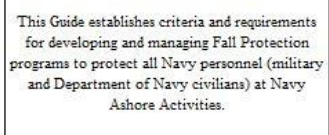 & Roof/ Deck & Fall Arrest System & 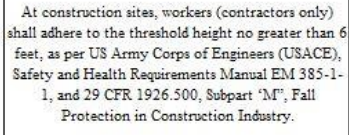 \\
\hline $\begin{array}{l}\text { Harmful Environments \& } \\
\text { Substances }\end{array}$ & $\begin{array}{l}\text { Coastal Carolina } \\
\text { University }\end{array}$ & $\begin{array}{l}\text { Hazardous Atmosphere - } \\
\text { Exhaust Prodvcing }\end{array}$ & Gasoline engines within a closed quarters & $\begin{array}{l}\text { Exhauss Producing } \\
\text { Asset }\end{array}$ & Ventilation & $\begin{array}{l}\text { Must not nun a asoline engine in closed quarters } \\
\text { without proper ventiliation }\end{array}$ \\
\hline
\end{tabular}




\section{Appendix K: Data Validation Interview Questions}

1. What is your current position?

a. What type of activities do you execute on a daily basis?

b. Do you manage any staff? If so, how many?

2. Appendix A.1 holds the list of safety inputs and protocols related to mitigating Falls

a. In terms of working at elevation, do you see any safety inputs that you may have in addition to the items listed? A safety input is an item that a FM worker would be required to know prior to working at elevation. Be sure to define each input and protocol as it relates to the research.

b. In regards to safety protocols, do you believe the list of mitigation techniques is comprehensive, related to each input?

c. Are any of the inputs or protocols unimportant? In other words, the input or protocol does not affect the FM worker while working at elevation?

3. Appendix A.2 holds the list of safety inputs and protocols related to mitigating Contact

a. In terms of working near mechanisms that could come into contact with a worker, do you see any safety inputs that you may have in addition to the items listed?

b. In regards to safety protocols, do you believe the list of mitigation techniques is comprehensive, related to each input?

c. Are any of the inputs or protocols unimportant? In other words, the input or protocol does not affect the FM worker while working at elevation?

4. Appendix A.3 holds the list of safety inputs and protocols related to mitigating Harmful Environments and Substances.

a. In terms of working in environments that are potentially dangerous to FM workers, do you see any safety inputs that you may have in addition to the items listed? Keep in mind that harmful environments and substances category incorporates air quality, oxygen deficiency, chemicals, electrical hazards, heat, and noise.

b. In regards to safety protocols, do you believe the list of mitigation techniques is comprehensive, related to each input?

c. Are any of the inputs or protocols unimportant? In other words, the input or protocol does not affect the FM worker while working at elevation?

5. Appendix B - Mind Mapping

a. As noted in the introduction, the safety inputs we have discussed were placed into a categorical format using a research technique known as mind mapping. At this point we will look through those mind maps and validate the categorical hierarchy and discuss where any new inputs discussed in this meeting should be placed.

6. Is there anything you wish you were asked in this interview that was not mentioned or wish to share? 


\section{Appendix L: Data Validation Interview Data Adjustments/Additions}

This section presents the complete list of Safety Inputs, Data Sourcing, and Protocols. The items list in black were identified during the Define Phase through Data Collection Interviews, Safety Literature, and FACE reports. The items listed in read were additions or adjustments made to the original list during the Measure Phase using the Data Validation Interviews.

\begin{tabular}{|c|c|c|c|c|}
\hline \multicolumn{5}{|c|}{ HARMFUL ENYIRONMENTS * SUBSTANCES } \\
\hline & & DATA SOURCI & & \\
\hline Safety Input & Typical Froduction Phase & Primary Developer & Method of Transfer & Khowledge : Relates to Safety Protocol \\
\hline \multirow{4}{*}{ Hazardous Energy } & \multirow{4}{*}{ Design } & \multirow{4}{*}{ Arch t Engineer } & \multirow{4}{*}{ Native BIMModel } & Lockoutt Tagout \\
\hline & & & & Hot SticksiMetering \\
\hline & & & & Permits \\
\hline & & & & Arc Flash Protection \\
\hline Elec. Disconnect Location(s) & Design & Archt Engineer & Native BIMModel & LockouttTagout \\
\hline \multirow{4}{*}{ Voltage } & \multirow{4}{*}{ Design } & \multirow{4}{*}{ Archt Engineer } & \multirow{4}{*}{ Native BIM Model } & Lockoutt Tagout \\
\hline & & & & Arc Flash Protection \\
\hline & & & & Permits \\
\hline & & & & Hot Sticks!Metering \\
\hline Appros. Disconnect Distance & Design & Archt Engineer & Native BIMModel & LockouttTagout \\
\hline \multirow{2}{*}{ Asbestos Potential } & \multirow{2}{*}{$\mathrm{FM}$} & \multirow{2}{*}{ FMStaff } & \multirow{2}{*}{ FM Safety Rielated Information } & Asbestos Management \\
\hline & & & & Respiratory Protection \\
\hline \multirow{2}{*}{ Environment Decibel Level } & \multirow{2}{*}{ Construction } & \multirow{2}{*}{$\begin{array}{l}\text { Manufacturer t } \\
\text { Subcontractor }\end{array}$} & \multirow{2}{*}{ Non-3D } & Hearing Protection + Noise Dampening \\
\hline & & & & Noise Monitoring \\
\hline & & & & SDStChemical Management \\
\hline & & & & Respiratory Protection \\
\hline & & & & Refrigerant Management \\
\hline Environmental Bir Puality & Desian & Archt Engineer & Native PIMModel & PCBManagement \\
\hline Environimentila Rir Quality & Design & Rrchitengineer & Native EIMModel & Silica Management \\
\hline & & & & Asbestos Management \\
\hline & & & & Lead Management \\
\hline & & & & Permits \\
\hline & & & & Hearing Protection + Noise Dampening \\
\hline & & & & Noise Monitoring \\
\hline & & & & SDStChemical Management \\
\hline & & & & Air Monitoring \\
\hline Manhole or Tank & Design & Arch tEngineer & Native BIM Model & Temperature Monitoring \\
\hline & & & & Valve Location(s) \\
\hline & & & & Confined Space Protocol \\
\hline & & & & Permits \\
\hline & & & & Ventilation \\
\hline & & & & Permits \\
\hline Oxygen Deficient I Orygen Enriched f & Design & Archid Enoineer & Matine BIMModel & Air Monitoring \\
\hline Carbon Monoside Environment & & & & Ventilation \\
\hline & & & & Respiratory Protection \\
\hline & & & & Air Monitoring \\
\hline Particulate Environment & Design & Archt Engineer & Native BIM Model & Ventilation \\
\hline & & & & Respiratory Protection \\
\hline Exhaust Producing Asset & $\mathrm{FM}$ & FMStaff & FM Safety Related Information & Yentilation \\
\hline & & & & Inflatable Valve \\
\hline Flooding Potential & Design & Archt Engineer & Native BIMModel & Value Location \\
\hline & & & & Safety Line \\
\hline Hazardous Asset Temperature & Design & Archt Engineer & Native BIMModel & Burn Mitigation i Frostbite Mitigation i' Hypo (Hyper)thermia \\
\hline Outdoor Enuiconment & Desing & Arous Enginer & Matiu PIM Moda & Weather Considerations - Heat, Cold, Precipitation, Wind \\
\hline Uutaoor Environiment & Design & Mron rengineer & 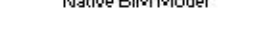 & Nature - Animals, Allergens, Plant Life \\
\hline Potentiat & EM & EMOU⿻丷木 & EMS Sutu Rolatorotion & LeadManagement \\
\hline LeadPotential & FM & FMStaff & FMS Safety Related Information & Respiratory Protection \\
\hline & & & & SDStChemical Management \\
\hline & & & & Air Monitoring \\
\hline Hazardous Chemical Production t & Decion & Arch Froineer & Matiue PlMModel & Secondary Containment \\
\hline Storage & Design & Arch tengineer & Native Bim Model & Ventilation \\
\hline & & & & Respiratory Protection \\
\hline & & & & Valve Location \\
\hline Radiation & Desian. & Arch Fnaineer & Native FlMModel & Monitoring \\
\hline & Design & Archt Engineer & & Radiation Management \\
\hline Polychlorinated Biphenyls (PCB) & FM & EMStaff: & FMSafetu Related Information & PCB Management \\
\hline Potential & $F|M|$ & & & Respiratory Protection \\
\hline
\end{tabular}




\begin{tabular}{|c|c|c|c|c|}
\hline \multicolumn{5}{|l|}{ FALLS } \\
\hline & & & & \\
\hline \multicolumn{5}{|c|}{ DATA SOURCING } \\
\hline Safety Input & Typical Production Phase & Primary Developer & Method of Transfer & Khowledge : Relates to Safety Protocol \\
\hline \multirow{3}{*}{ Maintenance Requires Lift System } & \multirow{3}{*}{ 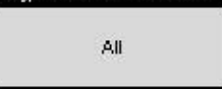 } & \multirow{3}{*}{ 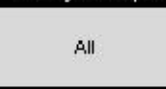 } & \multirow{3}{*}{$\begin{array}{l}\text { Native BIM Model, Non-3D, or FM } \\
\text { Safety Rielated Information }\end{array}$} & Lift System with Condition Assessment \\
\hline & & & & Working Height \\
\hline & & & & Fall Arrest System i Anchorage \\
\hline \multirow{5}{*}{ Roof t Deck } & \multirow{5}{*}{ Design } & \multirow{5}{*}{ Archt Engineer } & \multirow{5}{*}{ Native BIMModel } & Lift System \\
\hline & & & & Chemical Venting (Industrial Hygiene) \\
\hline & & & & Fall Arrest System I Anchorage \\
\hline & & & & Barricading / Notification \\
\hline & & & & Outdoor Environment \\
\hline \multirow{4}{*}{ Ledge i Hole } & \multirow{4}{*}{ Design } & \multirow{4}{*}{ Archt Engineer } & \multirow{4}{*}{ Native BIMModel } & Lift System \\
\hline & & & & Fall A.rrest System I Anchorage \\
\hline & & & & Safety Line \\
\hline & & & & Barricading + Notification \\
\hline
\end{tabular}

\begin{tabular}{|c|c|c|c|c|}
\hline \multicolumn{5}{|l|}{ CONTACT VITH I STRUCK BY } \\
\hline \multicolumn{5}{|c|}{ DATA SOURCING } \\
\hline Safety Input & Typical Production Phase & Primary Developer & Method of Transfer & Nhowhedge : Relates to Safety Protocol \\
\hline $\begin{array}{l}\text { Manual Crushing'Rotating/Slicing } \\
\text { Mechanism }\end{array}$ & Construction & $\begin{array}{l}\text { Manufacturert } \\
\text { Subcontractor }\end{array}$ & Native BIM Model or Non-3D & $\begin{array}{c}\text { Lockout/Tagout } \\
\text { Barricading ? Notification }\end{array}$ \\
\hline \multirow{4}{*}{$\begin{array}{l}\text { Automated CrushingtRotating/Slicing } \\
\text { Mechanism }\end{array}$} & \multirow{4}{*}{ Construction } & \multirow{4}{*}{$\begin{array}{l}\text { Manufacturer t } \\
\text { Subcontractor }\end{array}$} & \multirow{4}{*}{ Native BIM Model or Non-3D } & LockouttTagout \\
\hline & & & & Hot StickstMetering \\
\hline & & & & Permits \\
\hline & & & & Barricading / Notification \\
\hline \multirow{4}{*}{ Suction i Pressure Line } & \multirow{4}{*}{ Design } & \multirow{4}{*}{ Arch t Engineer } & \multirow{4}{*}{ Native BIMModel } & Safety Line \\
\hline & & & & Burn Mitigation ' Frostbite Mitigation I Hypo (Hyper)thermia \\
\hline & & & & Valve Location(s) \\
\hline & & & & Barricading/Notification \\
\hline Valve Location(s) & Design & Arch Engineer & Native BIMModel & Line Isolation \\
\hline \multirow{4}{*}{ Overhead Equipment } & \multirow{4}{*}{ All } & \multirow{4}{*}{ All } & \multirow{4}{*}{$\begin{array}{l}\text { Native BlM Model, Non-3D, or FM } \\
\text { Safety Rielated Information }\end{array}$} & Barricading + Notification \\
\hline & & & & Approach Vector \\
\hline & & & & Support Structure \\
\hline & & & & Equipment Weight \\
\hline Appron. Valve Location Distances & Design & Arch I Engineer & Native BIMModel & Line Isolation \\
\hline Asset Support Structure & Design & Archt Engineer & Native BIMModel & Approach Vector \\
\hline
\end{tabular}

\section{CHANGES}

Remove Paint from Lead Potential

Change "Self-Contained Breathing" to "Respitory Protection"

Add "Radiation" to Harmful Environment Inputs - Milliservients of radiation determine danger, Geiger Counter measures (20 mSV per year is a typical nuclear power plant worers exposure) Add Drygen Enriched

Add Cold Producing Asset \& Frostbite Mitigation I Hyppothermia Add silica danger

Temperature in Manhole

Add Nature Considerations to Outdoor Environment

Add Industrial Hygene or Chemical Venting at Roof

Address harmful temperature in suction or pressure lines

Support stucture for equipment

PCB Concerns Added

Secondary Containment added as a mitigation technique Added Approach Vectors

FOREACH INPUI

Verify Surroundings

Verify PPE Conditions

QualifiedPersonnel 


\section{Appendix M: Safety Input Relational Information as Formulated in the ASIT}

The following table presents the relationships formulated within the ASIT. A "Yes" response to any Primary Input requires values for the associated Dependent Inputs.

\begin{tabular}{|c|c|}
\hline Primary Input & Dependent Input Requiring Value \\
\hline \multirow{2}{*}{ Manual Crushing / Rotating / Slicing Mechanism } & Disconnect Mechanism Location (Manual Only) \\
\hline & Harmful Force $(\mathrm{Y} / \mathrm{N})$ \\
\hline \multirow{5}{*}{$\begin{array}{l}\text { Automated Crushing / Rotating / Slicing } \\
\text { Mechanism }\end{array}$} & Limit Switch Location (Where In Equipment) \\
\hline & Harmful Force $(\mathrm{Y} / \mathrm{N})$ \\
\hline & Disconnect Location (Panel Number) \\
\hline & $\begin{array}{l}\text { Secondary Feed Location (Panel Number or } \\
\text { None) }\end{array}$ \\
\hline & Approximate Disconnect Distance (in feet) \\
\hline \multirow{6}{*}{ Suction / Pressure Line } & Harmful Force $(\mathrm{Y} / \mathrm{N})$ \\
\hline & Valve Location (Valve Number) \\
\hline & $\begin{array}{l}\text { Secondary Valve Location (Valve Number or } \\
\text { None) }\end{array}$ \\
\hline & Line Size (in inches) \\
\hline & Approximate Valve Location Distance (in feet) \\
\hline & Harmful Temperature $(\mathrm{Y} / \mathrm{N})$ \\
\hline \multirow{3}{*}{ Overhead Equipment } & Maintenance Working Height (in feet) \\
\hline & Weight (in pounds) \\
\hline & $\begin{array}{l}\text { Support Structure Type (Platform, Threaded Rod, } \\
\text { Etc.) }\end{array}$ \\
\hline \multirow{8}{*}{ Environmental Air Quality Hazards Present } & Lead Present (Yes/No) \\
\hline & $\begin{array}{l}\text { Oxygen Deficient or Enriched / Carbon Monoxide } \\
\text { Environment (Yes/No) }\end{array}$ \\
\hline & Chemical No.1 Present \\
\hline & Chemical No. 2 Present (List Chemical or None) \\
\hline & Chemical No. 3 Present (List Chemical or None) \\
\hline & Particulate Present (Yes/No) \\
\hline & Asbestos Present (Yes/No) \\
\hline & PCBs Present (YES/NO) \\
\hline Environmental Decibel Level Hazard & Environmental Decibel Level (in dBA) \\
\hline \multirow{4}{*}{ Hazardous Energy / Live Current Present } & Disconnect Location (Panel Number) \\
\hline & $\begin{array}{l}\text { Secondary Feed Location (Panel Number or } \\
\text { None) }\end{array}$ \\
\hline & Approximate Disconnect Distance (in feet) \\
\hline & Voltage (in volts) \\
\hline \multirow{5}{*}{ Manhole /Tank / Confined Space } & Valve Location (Valve Number) \\
\hline & $\begin{array}{l}\text { Secondary Valve Location (Valve Number or } \\
\text { None) }\end{array}$ \\
\hline & Approximate Valve Location Distance (in feet) \\
\hline & Harmful Temperature $(\mathrm{Y} / \mathrm{N})$ \\
\hline & Flooding Potential Exists (Y/N) \\
\hline
\end{tabular}




\begin{tabular}{|l|l|}
\hline & Chemical Storage (Type or None) \\
\hline \multirow{3}{*}{ Hazardous Chemical Production Transmission } & Chemical No.1 Present \\
\cline { 2 - 2 } & Chemical No. 2 Present (List Chemical or None) \\
\cline { 2 - 2 } & Chemical No. 3 Present (List Chemical or None) \\
\hline Radiation & Milliservients of Radiation \\
\hline Heat / Cold Producing Asset & Harmful Temperature (Y/N) \\
\hline Maintenance Requires Lift System & Maintenance Working Height (in feet) \\
\hline
\end{tabular}

In a single instance, a "Yes" response to a Primary Input automates a "Yes" response to another Primary Input. If an asset has an "Automated Crushing/Rotating/Slicing Mechanism" this system will require a "Yes" to "Hazardous Energy."

\section{Primary Input}

Primary Input Requiring Value

Automated Crushing/Rotating/Slicing Mechanism

Hazardous Energy

In most cases Independent Inputs do not have Dependent Inputs and therefore are categorized separately, however, the below presets the single example of two Independent Inputs logically related. This example is also presented in Section 5.1 Phase I - Data Loading.

\section{Independent Input}

Located on Roof / Deck

\section{Independent Input Requiring Value}

Outdoor Environment (Auto YES)

In a single instance, a "Yes" response to a Dependent Input prompts the system to require a value for another Dependent Input. The Primary Input "Manhole / Tank / Confined Space" has a Dependent Input "Chemical Storage (Yes or None)." If a response of "YES" is given to this value, the system will prompt the user to input the chemicals present.

\section{Dependent Input}

Chemical Storage (Yes or None)

\section{Dependent Input Requiring Value}

Chemical No.1 Present

Chemical No. 2 Present (List Chemical or None)

Chemical No. 3 Present (List Chemical or None) 